\title{
2 Spatio-Temporal Dynamics of Reaction-Diffusion Patterns
}

\author{
Bernold Fiedler $^{1}$ and Arnd Scheel ${ }^{2}$ \\ 1 Freie Universität Berlin, FB Mathematik I, Arnimallee 2-6, 14195 Berlin, \\ Germany - fiedler@math.fu-berlin.de \\ 2 University of Minnesota, School of Mathematics, 206 Church St. S.E., \\ Minneapolis, MN 55455, USA - scheel@math.umn.edu
}

\subsection{Introduction and Overview}

In this survey we look at parabolic partial differential equations from a dynamical systems point of view. With origins deeply rooted in celestial mechanics, and many modern aspects traceable to the monumental influence of Poincaré, dynamical systems theory is mainly concerned with the global time evolution $\mathcal{T}(t) u_{0}$ of points $u_{0}$ - and of sets of such points - in a more or less abstract phase space $X$. The success of dynamical concepts such as gradient flows, invariant manifolds, ergodicity, shift dynamics, etc. during the past century has been enormous - both as measured by achievement, and by vitality in terms of newly emerging questions and long-standing open problems.

In parallel to this development, the applied horizon now reaches far beyond the classical sources of celestial and Hamiltonian mechanics. Applications areas today include physics, many branches of engineering, economy models, and mathematical biology, to name just a few. This influence can certainly be felt in several articles of this volume and cannot possibly be adequately summarized in our survey.

Some resources on recent activities in the area of dynamics are the book series Dynamical Systems I - X of the Encyclopedia of Mathematical Sciences [AnAr88, Si89, Ar\&al88, ArNo90, Ar94a, Ar93, ArNo94, Ar94b, An91, Ko02], the Handbook of Dynamical System [BrTa02, Fi02, KaHa02], the Proceedings [Fi\&al00], and the fundamental books [ChHa82, GuHo83, Mo73, KaHa95].

In the context of partial differential equations, the phase space $X$ of solutions $u=u(t, x)$ becomes infinite-dimensional: typically a Sobolev space of spatial profiles $u(t)=u(t, \cdot)$. More specifically, the evolution of $u(t)=$ $\mathcal{T}(t) u_{0} \in X$ with time $t$ is complemented by the behavior of the $x$-profiles $x \mapsto u(t, x)$ of solutions $u$. Such spatial profiles could be monotone or oscillatory; in $\operatorname{dim} x=1$ they could define sharp fronts or peaks moving at constant or variable speeds, with possible collision or mutual repulsion. Target pat- 
terns or spirals can emerge in $\operatorname{dim} x=2$. Stacks of spirals, which Winfree called scroll waves, are possible in $\operatorname{dim} x=3$. For a first cursory orientation in such phenomena and their mathematical treatment we again refer to [Fi02] and the article of Fife in the present volume, as well as the many references there.

For our present survey, we focus on the spatio-temporal dynamics of the following rather "simple", prototypical parabolic partial differential equation:

$$
u_{t}=D \Delta u+f(x, u, \nabla u)
$$

Here $t \geq 0$ denotes time, $x \in \Omega \subseteq \mathbb{R}^{m}$ is space and $u=u(t, x) \in \mathbb{R}^{N}$ is the solution vector. We consider $C^{1}$-nonlinearities $f$ and constant, positive, diagonal diffusion matrices $D$. This eliminates the beautiful pattern formation processes due to chemotaxis; see [JäLu92, St00], for example. Note that we will not always restrict $f$ to be a pure reaction term, like $f=f(u)$ or $f=$ $f(x, u)$. More general than that, we sometimes allow for a dependence of $f$ on $\nabla u$ to include advection effects. The domain $\Omega$ will be assumed smooth and bounded, typically with Dirichlet or Neumann boundary conditions, or else unbounded, typically $\Omega=\mathbb{R}^{m}$, an unbounded cylinder, or a half-space. At any rate, assumptions will be such that questions like global forward existence, uniqueness, and smoothness of weak solutions $u(t, x)$ for prescribed initial conditions $u_{0}(x)=u(t=0, x) \in X$ will not constitute a problem; see [He81, Pa83]. To emphasize the dynamical systems aspect of dependence on initial conditions $u_{0}(x)=u(t=0, x)$, we frequently write $\mathcal{T}(t) u_{0}=u(t)=$ $u(t, \cdot)$ for the strongly continuous solution semigroup $\mathcal{T}(t)$ of $(2.1)$ on $X$.

Within this general setting, we devote the following chapters to a discussion of increasingly specialized spatio-temporal patterns in increasing space dimensions $m=\operatorname{dim} x=1,2,3$. Our approach is motivated to some extent by classical dynamics in finite-dimensional spaces: while flows in $\operatorname{dim} X=2$ admit the beautifully simple Poincaré-Bendixson theory, present in any good textbook on ordinary differential equations, some of the complications arising in $\operatorname{dim} X=3$ are illustrated by the Lorenz attractor. Chapter 2.2 is devoted to single equations, $N=\operatorname{dim} u=1$, in one space dimension, $m=\operatorname{dim} x=1$. On bounded interval domains $\Omega$, the gradient-like dynamics is governed by a decreasing Lyapunov-functional, and by additional nodal properties, which were first discussed by Sturm; see Section 2.2.1. In Section 2.2.2 these Sturm properties provide a rather complete combinatorial characterization of the global PDE attractors $\mathcal{A}_{f}$ associated to (2.1). The case of a circle domain $\Omega=S^{1}$, in some sense an amphibium like $m=\operatorname{dim} x=1.5$, destroys the gradient-like Lyapunov structure, in Section 2.2.3, but preserves the Sturm structure. We sketch a new description of the global attractors for this case, which involves rotating waves on the circle and their heteroclinic connections. It is worth noting that individual orbits, but not the arbitrarily high-dimensional global attractor $\mathcal{A}_{f}$, do satisfy a Poincaré-Bendixson theorem in this case. Adding a linear, but nonlocal rank-1 perturbation can destroy this simple Morse-Smale 
structure completely, allowing for embeddings of arbitrarily complicated dynamics. Chapter 2.2 can also be read as a very classically-minded warm-up for the much more advanced discussion of gradient flows in the article of Fife in the present volume.

In the unbounded limit $\Omega=\mathbb{R}$, travelling waves can arise along with various instabilities; see Chapter 2.3. Stability considerations are greatly complicated - and phenomena as well as their mathematics greatly enriched - by the presence of continuous spectrum. As a prelude to our treatment of spiral wave bifurcations, in Chapter 2.5, we discuss dispersion relations and characterize Fredholm properties of linearizations at fronts in one space dimension in Section 2.3.1. The role of group velocities is then illustrated in a Hopf bifurcation from travelling waves caused by continuous spectrum in Section 2.3.2. We return to bounded but large domains $\Omega=(-L, L)$ in Section 2.3.3, where we investigate linear and nonlinear stability of fronts in the limit $L \rightarrow \infty$.

Chapter 2.4, dwelling on planar domains $\Omega \subseteq \mathbb{R}^{2}$, addresses the issue of spiral waves. Following [WiRo46], spiral patterns arise naturally in systems $(N=2)$ of excitable media type. As a link to geometric dynamics and mean curvature flow, we begin in Section 2.4.1 with a negative result: Archimedean rotating spirals cannot be described by a reduced model of curve-shortening type. Some nonlocal effects or extra input describing the tip motion, or other relics of the system-character $N=\operatorname{dim} n=2$ of the viscous approximation to the geometric model, have to be added. In Section 2.4.2, we develop a characterization of Archimedean spiral wave solutions to reaction-diffusion systems of $N$ species, valid for excitable and oscillatory media, but independent of singular limiting regimes such as fast relaxation kinetics or small amplitude oscillations. Our characterization ensures robustness of spiral waves when system parameters are varied. A bifurcation result in Section 2.4.3 provides us with an open class of reaction diffusion systems, where spiral waves are actually proved to exist.

Beyond rigidly rotating Archimedean spiral patterns, spiral waves generate a variety of spatio-temporal structures. In Chapter 2.5 we attempt a mathematical analysis based on bifurcation theory. In Section 2.5.1, we phenomenologically describe some of the patterns that may arise, building on experimental observations. We address in some detail meandering and drifting spirals, Doppler-induced super-spirals, and spiral breakup in the core and farfield. In Section 2.5.2 we outline a symmetry approach, which accurately describes observed meandering cycloid bifurcations from the unperturbed circular tip motion of rigidly rotating spiral wave patterns. The elementary Euclidean group $S E(2)$ of rotations and translations in the $x$-plane causes these motions by Hopf bifurcation transverse to the group orbit: a prime example of symmetry breaking under noncompact Lie groups. However, this simplistic analysis ignores the possible presence of continuous spectrum. In Section 2.5.3 we characterize spectral properties of spiral waves in large and 
unbounded domains. We focus on two major consequences of the presence of continuous spectrum in the unbounded domain. First, the shape of eigenfunctions to isolated eigenvalues is largely governed by the complex dispersion relation of the wavetrains in the farfield. Second, instabilities arising through the continuous spectrum come in two steps. At onset, the instability is convective in nature: perturbations decay pointwise, and the instability disappears after a possibly long transient. Only after driving the instability further by increasing the bifurcation parameter beyond threshold, the instability manifests itself in bounded domains. The discussion of spectra, here, is in strong analogy to the simpler setup in one space-dimension, Chapter 2.3. We conclude Chapter 2.5 relating spectral theory to the experimentally and numerically observed spiral wave patterns.

Chapter 2.6, where $m=\operatorname{dim} x=3$, discusses scroll wave dynamics. Following [Wi73], scroll waves can be viewed as stacks of rotating spirals with tip positions $x=z^{t}(\tau)$ aligned along filament curves $\tau \mapsto z^{t}(\tau) \in \Omega \subseteq \mathbb{R}^{3}$, for (most) fixed times $t$. Filaments $z^{t}$ can be knots with parameter $\tau \in S^{1}$. Moreover the spirals, which appear in spatial sections transverse to filaments $z^{t}(\tau)$ at $\tau$, define a local phase angle $\varphi^{t}(\tau) \in S^{1}$ there. We relate knotting orientations of filaments $z^{t}$, and "twist", alias the winding of $\varphi^{t}$, in Section 2.6.1. For generic initial conditions $u_{0}(x)=u(t=0, x)$, it is possible to describe all scenarios changing the knotting or linking topology of scroll wave filaments $z^{t}$; see section 2.6.2. We conclude with a discussion of some simulations of spatio-temporal scroll wave dynamics, in Section 2.6.3.

Acknowledgments. We are grateful for the hospitality of the program of the Twente Summer School and of the Seminars of DMV (German Mathematical Society) at Oberwolfach, where these results have first been compiled. This work was substantially supported by the Deutsche Forschungsgemeinschaft, in particular by the Sonderforschungsbereich "Complex Nonlinear Processes", DFG Priority Research Programs "Ergodic Theory, Analysis, and Efficient Simulation of Dynamical Systems" and "Analysis, Modeling, and Simulation of Multiscale Problems". For generous support of several research visits both authors are indebted to the Institute of Mathematics and its Applications (IMA) at Minneapolis. The first author would like to thank Regina Löhr for patiently typesetting quite a few versions of this manuscript. We are also indebted to many colleagues who have helped us along the way, most notably Sigurd Angenent, Pavol Brunovský, Klaus Ecker, Giorgio Fusco, Jack Hale, Jim Keener, Yannis Kevrekidis, John Mallet-Paret, Alexander Mikhailov, Waldyr Oliva, Peter Poláčik, Carlos Rocha, Björn Sandstede, Art Winfree, and Matthias Wolfrum.

Most of all, we feel deeply indebted to Willi Jäger, who untiringly created and maintained an inspiring work atmosphere at Heidelberg with such lasting influence on his scientific children and grandchildren. 


\subsection{One Space Dimension: Global Attractors}

\subsubsection{Lyapunov Functions, Comparison Principles, and Sturm Property}

In this chapter, we consider various special cases of reaction-advectiondiffusion systems of the general form

$$
u_{t}=D \Delta u+f(x, u, \nabla u),
$$

$\Omega \subseteq \mathbb{R}^{m}$, with $u \in \mathbb{R}^{N}, f \in C^{1}$, and Dirichlet, Neumann, or mixed boundary conditions; see (2.1). Mostly we will restrict our attention to the special case of a scalar equation, $N=1$, in one space dimension $m=1$. We begin our discussion, however, by recalling the larger, overlapping domains of validity for the concepts of gradient flows or gradient-like dynamics, as given by decreasing Lyapunov functions, and of monotonicity or comparison principles.

Lyapunov functions We first consider the variational structure which makes system (2.2) an $L^{2}$-gradient flow. We assume the vector nonlinearity $f=f(x, u)$ to be independent of the term $\nabla u$. In addition, we assume $f \in \mathbb{R}^{N}$ to be the gradient of a scalar potential $F=F(x, u)$ with respect to $u \in \mathbb{R}^{N}$,

$$
F_{u}(x, u)=f(x, u)
$$

Then the energy functional

$$
\mathcal{V}(u):=\int_{\Omega}\left(\frac{1}{2}\left(\sum_{j, k} \nabla u_{j} \cdot D_{j k} \nabla u_{k}\right)-F(x, u)\right) \mathrm{d} x
$$

is a Lyapunov function for system (2.2), if the positive definite diffusion matrix $D$ is symmetric. Indeed we observe the Lyapunov decay property:

$$
\frac{d}{d t} \mathcal{V}(u(t))=-\int_{\Omega} u_{t}^{2} \mathrm{~d} x
$$

is strictly negative, except at equilibrium solutions of the elliptic system $u_{t}=$ 0 . Of course we have to impose appropriate growth conditions on $f$ and choose an appropriate Sobolev space $X$ containing functions in $H^{1}(\Omega)$ with the chosen boundary conditions. Critical points of the energy functional $\mathcal{V}$ provide weak solutions of the associated elliptic equilibrium system $u_{t}=0$ and are amply studied; see for example the books [GiHi96a, GiHi96b, MaWi89, St90, Ze85], and the many references there.

By LaSalles invariance principle, the monotone decay (2.5) of the Lyapunov functional $\mathcal{V}$ implies $\mathcal{V} \equiv$ const. on the $\omega$-limit set

$$
\omega\left(u_{0}\right):=\left\{v \in X ; v=\lim u\left(t_{n}\right), \text { for some } t_{n} \rightarrow \infty\right\},
$$


of any bounded solution $u(t)=\mathcal{T}(t) u_{0} \in X$ with initial condition $u=u_{0}$ at $t=0$.

If we substitute the term $u_{t}$ in (2.2) by $A u_{t}$, with uniformly positive definite $N \times N$-matrices $A=A(x, u, \nabla u)$, and substitute $D \Delta u$ by an $x$-dependent matrix diffusion term $\operatorname{div}(D(x) \operatorname{grad} u)$ in divergence form, the same functional $\mathcal{V}(u)$ works as a valid Lyapunov function. Indeed, the Lyapunov decay property (2.5) remains valid with $u_{t}^{2}$ replaced by the nonnegative integrand $u_{t}^{T} A u_{t}$.

Another generalization of (2.4), due to [Al79], relaxes the very restrictive gradient condition (2.3) under Neumann boundary conditions and for $x$-independent $f=f(u)$. Since homogeneous $x$-independent solutions then satisfy the ODE system $\dot{u}=f(u)$, a gradient-like condition

$$
F_{u}(u) \cdot f(u)<0
$$

has to be imposed, except when $f(u)=0$ provides a spatially homogeneous equilibrium already. An additional convexity condition then ensures that $\mathcal{V}$ becomes a Lyapunov function, decreasing strictly along any non-equilibrium solutions.

In the case of scalar equations, $N=1$, the gradient condition (2.3) is of course trivially satisfied. Even in the one-dimensional case $m=1$, however, with periodic boundary conditions $x \in S^{1}=\mathbb{R} / \mathbb{Z}$, Lyapunov functions may fail to exist, if we let the nonlinearity $f=f\left(x, u, u_{x}\right)$ depend on the gradient term $u_{x}$ :

$$
u_{t}:=u_{x x}+f\left(x, u, u_{x}\right) .
$$

In fact a particularly simple example arises for $x$-independent nonlinearities $f=f\left(u, u_{x}\right)$. Note $S^{1}$-equivariance with respect to $x$-shift in that case: with $u(t, x)$, also $u\left(t, x-x_{0}\right)$ is a solution of (2.8), for any fixed $x$-shift by $x_{0} \in S^{1}$. We can then find rotating wave solutions of the form

$$
u(t, x)=U(x-c t),
$$

$c \neq 0$, which are provided by any nonstationary 1-periodic solution of the second order ODE

$$
U^{\prime \prime}+c U^{\prime}+f\left(U, U^{\prime}\right)=0 .
$$

Such solutions $U$ are easily constructed, for suitable nonlinearities $f$; for example the class (2.10) contains the van der Pol oscillator. See [AnFi88] and Section 2.2.3 for more details. Clearly the existence of rotating waves (2.9) with time period $1 / c$ contradicts the existence of a Lyapunov function $\mathcal{V}$ which would have to strictly decrease with time. Similar constructions work for thin annulus domains $\Omega \subset \mathbb{R}^{2}$ and for disks. In fact we could just transform any system with $f=f(u)$ to coordinates rotating at uniform angular 
speed $c \neq 0$. Any equilibrium solution which is not rotationally symmetric will then provide a rotating wave solution for suitable $f=f(x, u, \nabla u)$.

In view of such examples it is therefore perhaps surprising, and not widely known, that a Lyapunov functional of the form

$$
\mathcal{V}(u)=\int_{\Omega} \gamma\left(x, u, u_{x}\right) \mathrm{d} x
$$

does exist for (2.8), in the case of an interval domain $\Omega=(0,1)$ with separated boundary conditions of Dirichlet, Neumann, or Robin type; see [Ze68, Ma78]. See also the beautiful construction in [Ma88]. Again the Lyapunov decay property (2.5) remains valid with $u_{t}$ replaced by $A u_{t}$, for some uniformly positive $A=A\left(x, u, u_{x}\right)$.

The construction of $\gamma$ by Matano in [Ma88] is particularly appealing: $\gamma$ is constructed via solutions of subsidiary semilinear hyperbolic equations, which defy periodicity conditions in $x$ and thus quite appropriately fail to exist under periodic boundary conditions $\Omega=S^{1}$. For analogous constructions of Lyapunov functions in the spatially discrete case of Jacobi systems and for certain graphs of neural networks see [FiGe98, FiGe99].

Comparison principles Monotonicity properties, alias comparison principles, are the second major structural property of reaction-advection-diffusion equations (2.2). We refer to [Po02] for a recent survey and only sketch the main line of thought, briefly. Let $u_{0}(x), \tilde{u}_{0}(x)$ be two initial conditions in our phase space $X \subseteq C^{0}(\bar{\Omega})$, with associated (global) solutions $u(t)=\mathcal{T}(t) u_{0}, \tilde{u}(t)=\mathcal{T}(t) \tilde{u}_{0} \in X$. The (strong) monotonicity property, or parabolic comparison principle, states that

$$
u_{0} \leq \tilde{u}_{0} \Rightarrow u(t)<\tilde{u}(t), \text { for all } t>0 .
$$

Here $\leq$ indicates that $u_{0}(x) \leq \tilde{u}_{0}(x)$, for all $x \in \Omega$, whereas $<$ indicates pointwise strict ordering inside $\Omega$. See [Sm83, Wa70, PrWe67] for proofs of this property in a scalar parabolic context $N=\operatorname{dim} n=1, m=\operatorname{dim} x \geq 1$. The proof is based on the elementary observation that the difference $w:=$ $\tilde{u}-u$ of the two solutions satisfies a nonautonomous linear parabolic equation of the form

$$
w_{t}=D \Delta w+a(t, x) w+b(t, x) \nabla w
$$

for suitable coefficients $a, b$ which depend on the solutions $u, \tilde{u}$. For example

$$
a=\int_{0}^{1} f_{u}(x, u+\vartheta(\tilde{u}-u), \nabla u+\vartheta(\nabla \tilde{u}-\nabla u)) \cdot(\tilde{u}-u) \mathrm{d} \vartheta
$$

where $\tilde{u}, u$ have to be evaluated at $(t, x)$. Note $w_{0} \geq 0$. From (2.13) it is immediately clear that any nondegenerate local $x$-minimum $w\left(t_{0}, x_{0}\right)=0$, should it ever develop at some time $t=t_{0}$, immediately retracts to locally 
strictly positive values. Indeed (2.13) then implies $w_{t}=\Delta w>0$ at $\left(t_{0}, x_{0}\right)$. The rest is parabolic technique.

A very successful general framework for monotonicity properties has been initiated by Hirsch and Matano; see [Hi83, Hi85, Hi88, Ma86, Ma87]. For example, Hirsch has proved that most initial conditions, in the Baire sense, give rise to solutions $u(t)$ which, if bounded, converge to some equilibrium in $X$ for $t \rightarrow+\infty$. In particular this implies that any hyperbolic periodic solution of a (strongly) monotone system is necessarily unstable. Let us briefly indicate a proof of this latter fact. Indeed the compact Floquet-operator is given by $w_{0} \mapsto w(p) \in X$, with linearization along the periodic orbit of the form (2.13) and with $p$ denoting the time period. The compact Floquet-operator therefore preserves positivity. By Perron-Frobenius theory, any eigenvalue of maximal modulus possesses a positive eigenfunction. For a periodic solution $u(t)$ with trivial Floquet multiplier 1, the trivial Floquet eigenfunction $w:=u_{t}$ cannot possibly be positive. By hyperbolicity, the periodic solution therefore possesses another Floquet multiplier outside the unit circle and with positive eigenfunction. Instability ensues. For an excellent survey of more recent, deeper results in this context, in particular concerning generic convergence to subharmonicity for time-periodically forced monotone systems, see [Po02].

From a pure dynamics point of view, monotonicity is not a serious restriction. As Smale has pointed out, any Lipschitz flow in $\mathbb{R}^{n}$ can be embedded into $u \in \mathbb{R}^{n+1}$ - normally unstable, of course - such that the resulting system is monotone. Just view $\mathbb{R}^{n}$ as the hyperplane $u_{1}+\cdots+u_{n+1}=0$ and add the fast scalar equation $\varepsilon \dot{v}=v$ in the normal direction, for sufficiently small $\varepsilon>0$. The resulting system will be monotone with respect to the componentwise order $\leq$ on $\mathbb{R}^{n+1}$. Of course, this argument somewhat neglects the spatial aspects of dynamics which are so relevant for PDEs. Moreover, instability does not necessarily indicate invisibility or irrelevance. Unstable solutions characterize basin boundaries of stable objects. And sometimes their time scales of instability, which are not at all affected by the monotonicity requirement, may comfortably exceed the life times of their observers.

Sturm property, revisited We now return to the scalar case, $N=$ $\operatorname{dim} u=1$, in one space-dimension, $m=\operatorname{dim} x=1$ with nonlinearity $f=f\left(x, u, u_{x}\right)$, as specified in (2.8) above. We admit separated boundary conditions, $x \in(0,1)$, with a resulting gradient-like flow, as well as periodic boundary boundary conditions, $x \in S^{1}=\mathbb{R} / \mathbb{Z}$, which defy Lyapunov functionals and admit nontrivial time-periodic solutions. In either case, there exists a very powerful refinement of the above monotonicity structure: the Sturm property. For any continuous function $w:[0,1] \rightarrow \mathbb{R}$ let the zero number $z(w) \leq \infty$ denote the number of strict sign changes of $w$. In other words $z$ is the supremum of all $n$, for which we can find $x_{1}<\cdots x_{n}$ such that the nonzero signs of $w\left(x_{i}\right)$ alternate. Let $w=w(t, x)$ denote a solution (2.13) in one space dimension

$$
w_{t}=w_{x x}+b(t, x) w_{x}+a(t, x) w .
$$


Clearly $z=z(w(t, \cdot))$, the number of sign changes of the $x$-profiles, may then depend on $t$. As far back as 1836, Sturm has observed that the zero number

$$
z(w(t, \cdot)) \underset{t}{\searrow}
$$

is nonincreasing with $t$, at least for time-independent $a, b$. See [St36] and also [Po33].

Sturm has used his beautiful observation to prove that any nontrivial linear combination $\varphi$ of eigenfunctions $\varphi_{j}, k \leq j \leq \ell$, of any Sturm-Liouville problem

$$
\lambda \varphi=\varphi_{x x}+b(x) \varphi_{x}+a(x) \varphi
$$

on the interval $0 \leq x \leq 1$ possesses at least $k$ and at most $\ell$ sign changes:

$$
k \leq z(\varphi) \leq \ell
$$

Here we number the eigenfunctions $\varphi_{j}$ such that $z\left(\varphi_{j}\right)=j$. Defining $w(t, \cdot)$ to be the explicit exponential solution of (2.15), for all $t \in \mathbb{R}$, with initial condition $w=\varphi$ at $t=0$, we indeed see how $w /|w|$ approaches eigenfunctions $\varphi_{j \pm}$, for $t \rightarrow \pm \infty$. Invoking (2.16), we obtain for some large enough $t>0$ that

$$
\ell \geq j_{-}=z(w(-t, \cdot)) \geq z(w(0, \cdot))=z(\varphi) \geq z(w(t, \cdot))=j_{+} \geq k .
$$

This single line proves $(2.18)$.

It is easy to prove that (2.16) indeed holds, locally, at a nondegenerate double zero

$$
w=w_{x}=0 \neq w_{x x}, \quad \text { at }\left(t_{0}, x_{0}\right) .
$$

Then (2.15) implies sign $w_{t}=\operatorname{sign} w_{x x}$, and an elementary argument shows that the zero number $z(w(t, \cdot))$ drops by 2 , locally at $\left(t_{0}, x_{0}\right) \in(0, \infty) \times[0,1]$. See also Figure 2.1(a). It is less obvious that $z$ also drops strictly at a triple, or multiple zero, where only $w=w_{x}=0$ is required; see Figure 2.1(b).

Proposition 2.2.1 Let $w(t, x) \not \equiv 0$ solve the linear, nonautonomous equation (2.15) in one space dimension and with separated or periodic boundary conditions. Let the zero number $z(w(t, \cdot))$ denote the number of strict sign changes of the $x$-profiles $x \mapsto w(t, x), x \in[0,1]$.

Then $w$ satisfies the Sturm property, that is

(i) $z(w(t, \cdot))$ is nonincreasing with time $t$;

(ii) $z(w(t, \cdot))$ drops strictly, whenever $w(t, \cdot)$ possesses any multiple zero at any $x \in[0,1]$;

(iii) $z(w(t, \cdot))$ is finite, for any positive $t$. 


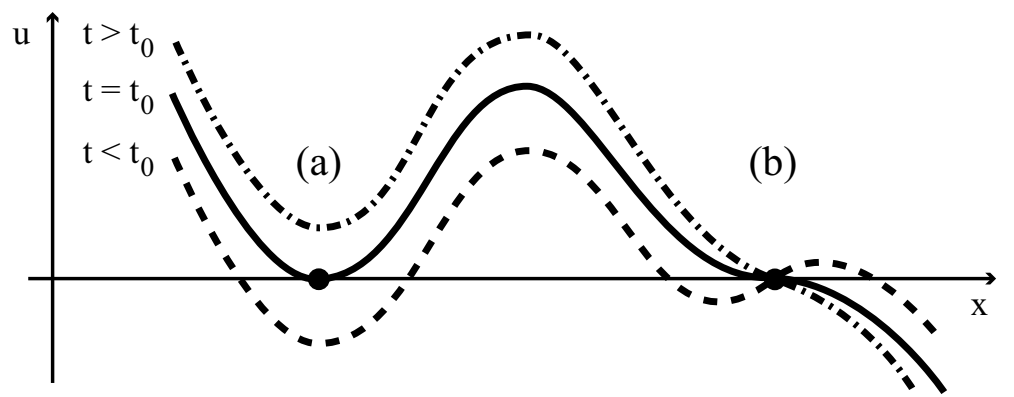

Fig. 2.1. Local dropping of $z(w(t, \cdot))$ at (a) a double zero, (b) a triple zero of $z\left(w\left(t_{0}, \cdot\right)\right)$.

Note how the trivial case $z=0$ of sign definite $w$ corresponds to the monotonicity or comparison principle (2.12) discussed above. For solutions $w(t, x)$ which are analytic in $(t, x)$, the proposition can be proved by Newton's polygon. In the autonomous case, this was Sturm's proof. For a modern version see [AnFi88]. The definitive version of this result, under rather mild regularity assumptions on $a, b$, was given by [An88]. We have explained above the renewed interest in the Sturm property for nonautonomous coefficients $a, b$ : any difference $w=\tilde{u}-u$ of two solutions $u, \tilde{u}$ of the semilinear reaction-advection-diffusion equation (2.8) satisfies (2.15) and thus possesses the Sturm property.

We will see in the next two sections, how this simple additional Sturm property has enormous consequences for the description of the global spatiotemporal dynamics of (2.8). It has definitely been due to the deeply inspiring insight of Matano [Ma82], that these enormous nonlinear consequences have been first realized. We just illustrate the beginnings of his contributions with two further remarks here.

First, Matano's original idea was based on using the lap number, $z\left(u_{x}(t, \cdot)\right)$, to characterize properties of solutions $u(t, \cdot)$ for nonlinearities $f=f\left(u, u_{x}\right)$ independent of $x$. Clearly this amounts to a choice $w=u_{x}$ and of course $w$ satisfies the linearized equation (2.8), which takes the form (2.15). In particular, the Sturm property implies that the $x$-profile $u(t, \cdot)$ decomposes into finitely many intervals of strict monotonicity, for any positive $t$.

Second, we can use the zero number $z$ and the Sturm property to prove convergence of any bounded solution $u(t, x)$ to a single equilibrium, for $t \rightarrow$ $\infty$, for $x \in[0,1]$, and under Neumann boundary conditions. Since $w=u_{t}$, like $u_{x}$, satisfies the linearized equation (2.15), even for nonlinearities $f=$ $f\left(x, u, u_{x}\right)$ which do depend on $x$, the Sturm property implies that all zeros of $u_{t}$ must be simple, eventually, for large $t \geq t_{0}$. But $u_{t x} \equiv 0$ at $x=0$, due to the Neumann boundary condition. Therefore $u(t, x=0)$ must be eventually monotone, hence convergent, and hence constant on the $\omega$-limit set $\omega(u) \subseteq X$. Therefore $u_{t} \equiv u_{t x} \equiv 0$ on $\omega(u)$ and $z\left(u_{t}\right)$ would have to drop there, all the 
time. Well, can it? No way: by the Sturm property, again, we see that $u_{t} \equiv 0$ on $\omega(u)$. Hence $\omega(u)$ consists entirely of equilibria $v=v(x)$. But $v(x=0)=$ $\lim _{t \rightarrow \infty} u(t, x=0)$ is fixed on $\omega(u)$. Together with the Neumann boundary condition $v_{x}(x=0)=0$, this specifies $v \in \omega(u)$ uniquely: the $\omega$-limit set always consists of just a single equilibrium. Note how this argument provides a second proof of the gradient-like behavior of the scalar, one-dimensional reaction-advection-diffusion equation (2.8), which is completely independent of the approach by Zelenyak and Matano [Ze68, Ma78, Ma88] via Lyapunov functions (2.11).

\subsubsection{Sturm Attractors on the Interval}

In this section and the following one we describe some progress during the last 15 years in our understanding of global attractors of the scalar, onedimensional reaction-advection-diffusion equation

$$
u_{t}=u_{x x}+f\left(x, u, u_{x}\right) .
$$

The present section addresses the interval case $0<x<1$ under separated boundary conditions; to be completely specific we consider Neumann boundary

$$
u_{x}=0 \quad \text { at } \quad x=0,1 .
$$

For periodic boundary conditions $x \in S^{1}=\mathbb{R} / \mathbb{Z}$ and nonlinearities $f=$ $f\left(u, u_{x}\right)$ see Section 2.2.3.

Global attractors For an excellent recent survey on the theory of global attractors, including topics like dimension estimates, inertial manifolds, determining modes and applications to retarded functional differential equations, damped hyperbolic wave equations, and the gradient-like parabolic equations studied here, we refer to the book by Chepyzhov and Vishik [ChVi02], which includes the nonautonomous case, and to the article by Raugel [Ra02]. For earlier work on global attractors see for example the books [Ha88, Te88, La91, BaVi92] and the many references there.

Abstractly, the concept is the following. Recall that $\mathcal{T}(t) u_{0}:=u(t)$ denotes the solution semigroup of (2.21) on a suitable Banach space $X$ of $x$ profiles $u(t)=u(t, \cdot) \in X$. We require $f \in C^{2}$ to be point dissipative, that is, there exists a (large) ball in $X$, in which any solution $\mathcal{T}(t) u_{0}$ stays eventually, for all $t \geq t_{0}=t_{0}\left(u_{0}\right)$. Explicit sufficient conditions on $f$ require, for example,

$$
f(x, u, 0) \cdot u<0
$$

for all sufficiently large $|u|$, along with uniformly subquadratic growth of $f$ in the variable $u_{x}$. Condition (2.23) provides an $L^{\infty}$-bound for $u$, whereas 
subquadratic growth prevents blow-up of $u_{x}$. Since $\mathcal{T}(t)$ is a compact, point dissipative semigroup, in the sense of the references quoted above, the global attractor $\mathcal{A}=\mathcal{A}_{f}$ of $(2.21)$ can be equivalently characterized as follows:

$$
\begin{aligned}
\mathcal{A} & =\text { the smallest set attracting all bounded sets } \\
& =\text { the largest compact invariant set } \\
& =\text { the set of all bounded solutions } \mathcal{T}(t) u_{0} \in X, t \in \mathbb{R}
\end{aligned}
$$

Here attractivity is understood in the sense that $\mathcal{T}(t) \mathcal{B}$ stays in any arbitrarily small $\delta$-neighborhood of $\mathcal{A}$, for any bounded set $\mathcal{B} \subset X$ of initial conditions and for all $t \geq t_{0}(\mathcal{B}, \delta)$. Invariance is understood in both forward and backward time. Negative time invariance of $\mathcal{A}$ requires the existence of a past history $u(-t) \in \mathcal{A}, t \geq 0$, of $u_{0}$, such that $\mathcal{T}(t) u(-t)=u_{0}$ for all $t>0$. Similarly, the set of all bounded solutions is understood to consist of precisely those $u_{0}$ which possess a bounded past history, in addition to $\mathcal{T}(t) u_{0}$ being bounded uniformly for $t \geq 0$. We need not really bother about the mind-boggle of huge fans of past histories of $u_{0}$ here, because backwards uniqueness for parabolic equations implies that past histories are uniquely determined.

Under suitable dissipativeness conditions on the nonlinearity $f$, the above statements hold true in complete generality (2.1), including systems $u \in$ $\mathbb{R}^{N}, N \geq 1$, higher-dimensional smoothly bounded $\Omega \subseteq \mathbb{R}^{m}, m \geq 1$, or the circle case $\Omega=S^{1}$. The gradient-flow property of $(2.21)$ with Neumann boundary conditions (2.22), however, allows for an even more specific characterization of the global attractor:

$$
\mathcal{A}=\mathcal{E} \cup\{\text { heteroclinics }\} .
$$

Here $\mathcal{E}=\left\{u_{t}=0\right\} \subset X$ denotes the set of equilibria. We call $u_{0} \in X$ heteroclinic, if there exists a solution $u(t), t \in \mathbb{R}$, including a past history, such that the distinct $\alpha$ - and $\omega$-limit sets of $u_{0}$ for $t \rightarrow \pm \infty$ are both equilibria. We have already seen in section 2.1 how these sets will consist of a single equilibrium $U_{ \pm}$, respectively, as $t \rightarrow \pm \infty$. The Lyapunov function $\mathcal{V}$, which only exists under separated boundary conditions, implies $\mathcal{V}\left(U_{+}\right)<\mathcal{V}\left(U_{-}\right)$ and hence $U_{+} \neq U_{-}$: heteroclinicity. In fact homoclinic solutions with $U_{+} \equiv$ $U_{-} \not \equiv u(t)$ are possible for $x \in S^{1}$; see [SaFi92] and the remarks at the end of Section 2.2.3.

Sturm attractors and Sturm permutations For simplicity of presentation, we henceforth assume that all equilibria $U \in \mathcal{E}$ are hyperbolic: all eigenvalues $\lambda$ of the Sturm-Liouville eigenvalue problem (2.17), arising by linearization at $U$, are nonzero. Such an assumption holds for most nonlinearities $f$, in a Baire sense: zero has to be a regular value of $u \mapsto u_{x x}+f$. Hyperbolicity implies that equilibria $U$ are locally isolated and, by compactness of $\mathcal{E} \subseteq \mathcal{A}$, finite in number,

$$
\mathcal{E}=\left\{U_{1}, \cdots, U_{n}\right\} .
$$


Moreover, they are accompanied by a local saddle structure, much in the geometric spirit of ODEs, characterized by local stable and unstable manifolds $W_{j}^{s}, W_{j}^{u}$ at each equilibrium $U_{j}$. These invariant manifolds consist of those $u_{0} \in X$ which remain in a neighborhood of $U_{j}$ and converge to that same equilibrium in forward resp. backward time. Their tangent spaces at $U_{j}$ are of course spanned by the Sturm-Liouville eigenfunctions $\varphi$ of (2.17) with negative resp. positive eigenvalues $\lambda$. The unstable manifolds $W_{j}^{u}$ in fact extend forward, globally, by the solution semigroup $\mathcal{T}(t), t \geq 0$. Noting that trivially $U_{j} \in W_{j}^{u}$, even if $U_{j}$ is stable, the above characterization (2.25) of the global attractor therefore takes the equivalent form

$$
\mathcal{A}=\bigcup_{j=1}^{n} W_{j}^{u}
$$

So much for generalities. As to the specific determination of global attractors $\mathcal{A}_{f}$ associated to nonlinearities $f$ of the parabolic equation (2.21), and in fact for any gradient-like system, several basic questions arise:

Q0: What are the equilibria $U_{1}, \cdots, U_{n}$ ?

Q1: Given all equilibria, which pairs possess a heteroclinic orbit?

Q2: Given dissipative nonlinearities $f, \tilde{f}$, how can we determine whether or not their global attractors $\mathcal{A}_{f}, \mathcal{A}_{\tilde{f}}$ coincide?

Q3: How many different global attractors with $n$ equilibria are there?

Q4: What is the geometry of the global attractors?

Note that Q0 is an ODE question concerning the scalar second order equation

$$
0=U_{x x}+f\left(x, U, U_{x}\right)
$$

with Neumann boundary conditions. See [Sc90] for a detailed analysis of the case $f=f(U)$. In contrast, question Q1 - Q4 address the PDE (2.2). We will now summarize some results which show how some essentially combinatorial information on the ODE question Q0 is sufficient to answer the PDE questions Q1 and Q2. In particular we will make precise what we mean by "coinciding" attractors. The challenging questions $\mathbf{Q 3}, \mathbf{Q 4}$, must remain open at this time.

Since our answers to Q0 - Q2 for the parabolic equation (2.2) are crucially determined by the Sturm property of solutions, Proposition 2.2.1, we call the associated global attractors Sturm attractors.

The combinatorial ODE information on the equilibria $U_{1}, \cdots, U_{n}$ has been ingeniously distilled by [FuRo91]. Under Neumann boundary conditions, let the equilibria $U_{1}, \cdots, U_{n}$ be numbered according to the strict ordering

$$
U_{1}<U_{2}<\cdots<U_{n}, \quad \text { at } x=0 .
$$

Indeed $U_{x}=0$ at $x=0$, together with the second order type of the equilibrium equation (2.28), implies that $i=j$, if $U_{i}=U_{j}$ at $x=0$. Now define 
the Sturm permutation $\pi=\pi_{f} \in S_{n}$ by the corresponding, possibly different ordering at the other boundary point $x=1$ :

$$
U_{\pi(1)}<U_{\pi(2)}<\cdots U_{\pi(n)}, \quad \text { at } x=1
$$

To get used to Sturm permutations, let us quickly prove that $\pi(1)=1$, $\pi(n)=n$. Indeed choose a very large positive or negative initial condition $u_{0}^{ \pm}$: pointwise above resp. below any equilibrium $U_{j}$. By dissipativeness, the solutions $\mathcal{T}(t) u_{0}^{ \pm}$must then each converge to some equilibrium $U^{ \pm}$. By monotonicity (2.12), alias nonincrease of $z\left(u(t)-U_{j}\right)=0$, that equilibrium must still lie above, resp. below, all other equilibria, pointwise. Hence $U^{+}=$ $U_{n}$ and $U^{-}=U_{1}$. This proves $\pi(1)=1, \pi(n)=n$.

Question Q2 was answered by [FiRo00] as follows.

Theorem 2.2.2 Let $f, \tilde{f}$ be dissipative nonlinearities in (2.21), (2.22) such that their Sturm permutations $\pi_{f}$ and $\pi_{\tilde{f}}$ coincide. Then the associated global attractors, the $\mathrm{Sturm}$ attractors $\mathcal{A}_{f}$ and $\mathcal{A}_{\tilde{f}}$, are $C^{0}$ orbit equivalent. In symbols

$$
\pi_{f}=\pi_{\tilde{f}} \quad \Rightarrow \quad \mathcal{A}_{f} \cong \mathcal{A}_{\tilde{f}}
$$

We recall that $C^{0}$ orbit equivalence is given by a homeomorphism $H$ : $\mathcal{A}_{f} \rightarrow \mathcal{A}_{\tilde{f}}$, in the topology of $X$, which maps $f$-orbits in $\mathcal{A}_{f}$ onto $\tilde{f}$-orbits in $\mathcal{A}_{\tilde{f}}$, preserving time direction.

We sketch some of the ingredients to the proof of this theorem. Of central importance in the Morse-Smale property on attractors $\mathcal{A}$ with hyperbolic equilibria: all intersections $W_{i}^{u} \cap W_{j}^{s}$ of stable and unstable manifolds are transverse, automatically, due to the Sturm property of the semiflow. This striking fact was discovered independently by [An86] and [He85]. An indirect zero number argument runs as follows. Consider a heteroclinic orbit $u(t) \in W_{k}^{s}$ from $U_{j}$ to $U_{k}$. If transversality failed, we could choose a nontrivial solution $\psi(t)$ of the adjoint linearization, along $u(t)$, perpendicular to the tangent spaces

$$
T_{u(t)}:=T_{u(t)} W_{j}^{u}+T_{u(t)} W_{k}^{s} .
$$

Letting $t \rightarrow-\infty$, we then see

$$
u(\psi(t)) \geq i_{j}, \quad \text { for all } t .
$$

Here $i_{j}$ denotes the unstable dimension, alias the Morse index at the hyperbolic equilibrium $U_{j}$. Indeed the adjoint linearization is parabolic in backwards time and hence $z(\psi(t))$ increases with $t$. Sturm-Liouville theory then shows the inequality (2.33) "at" $t=-\infty$, because $\psi(t) \perp T_{u(t)} W_{j}^{u}$. A similar argument at $t=+\infty$ proves

$$
z(\psi(t))<i_{k}, \quad \text { for all } t .
$$


Together, this implies $i_{j}<i_{k}$ for the Morse indices of the source $U_{j}$ and the target $U_{k}$. Arguing for the heteroclinic tangent $u_{t}$ itself, on the other hand, and using that $u_{t} /\left|u_{t}\right|$ converges to eigenfunctions for $t \rightarrow \pm \infty$, by [BrFi86], we see that the Sturm property for the linearization along $u(t)$ implies

$$
i_{k} \leq z\left(u_{t}(t)\right)<i_{j}, \quad \text { for all } t .
$$

This is a clear contradiction to $i_{j}<i_{k}$, and hence proves the Morse-Smale property.

For dynamical systems on compact manifolds, Palis and Smale have proved structural stability of Morse-Smale systems; see [Pa69, PaSm70]. Infinite-dimensional versions are due to Oliva; see [O102]. In our setting this implies the claim of Theorem 2.2.2, provided that $\tilde{f}$ is near $f$ in the $C^{2}$ topology. It also proves the claim, if we can find a homotopy $f^{\tau}$ from $f^{0}=f$ to $f^{1}=\tilde{f}$, in the class of dissipative $C^{2}$-nonlinearities and parabolic equations (2.21), such that all equilibria in $\mathcal{E}_{\tau}=\mathcal{E}_{f \tau}$ remain hyperbolic throughout this homotopy. Unfortunately, however, it is still not clear to us whether or not such a homotopy $f^{\tau}$ exists.

We have circumvented this difficulty by discretization within the class of Jacobi systems, also studied by Oliva, see [O102]. These are finite tridiagonal nonlinear systems

$$
\dot{u}_{i}=f_{i}\left(u_{i-1}, u_{i}, u_{i+1}\right),
$$

$i=1, \cdots, \ell$, such that the off-diagonal partial derivatives of all $f_{i}$ are strictly positive everywhere. Boundary conditions like $u_{0}:=u_{1}, u_{\ell+1}:=u_{\ell}$ have to be imposed. Counting strict sign changes along the discrete positions $1 \leq i \leq \ell$, instead of $0 \leq x \leq 1$, provides a Sturm property which is completely analogous to the continuous parabolic case. The Morse-Smale property follows, likewise, from hyperbolicity of equilibria. Finite difference discretization of (2.21) for $f$ and $\tilde{f}$, for example, leads to Jacobi systems $\ell$ large enough to ensure structural stability of the respective finite-dimensional global attractors under discretization.

But not all Jacobi systems are discretizations of parabolic equations. The additional freedom thus gained, adorned with an artificial unstable suspension trick which quadruples the dimension of the system, is sufficient to find a homotopy $f^{\tau}$ as described above, but on the discrete level. This completes our sketch of the proof of Theorem 2.2.2.

As an aside we note that the class A ${ }^{J}$ of (Morse-Smale) Sturm attractors of Jacobi systems coincides with the same class $\mathrm{A}^{P}$ for one-dimensional parabolic equations. Discretization shows $\mathrm{A}^{J} \subseteq \mathrm{A}^{P}$. The combinatorial characterization of possible Sturm permutations, given in Theorem 2.2.4 below, shows that $\mathrm{A}^{J} \subseteq \mathrm{A}^{P}$; see [FiRo00]. Similarly, the class $\mathrm{A}^{P}$ is independent of the choice of separated boundary conditions; see [Fi96]. Moreover, this suggests extensions to quasilinear or nonlinear parabolic equations: as long 
as finite-difference discretization to Jacobi systems prevails, no new attractors are to be expected. An open question, however, is realization of Sturm attractors $\mathcal{A}$ by Jacobi systems of minimal dimension. Let

$$
\ell:=\operatorname{dim} \mathcal{A}=\max _{1 \leq j \leq n} i_{j}
$$

denote the maximal Morse-index of equilibria $U_{j} \in \mathcal{A}$. Is it then possible to realize that same attractor $\mathcal{A}$, up to $C^{0}$ orbit equivalence, by a Jacobi system (2.36) of dimension only $\ell$ ? A positive answer would provide the ultimate "qualitative" discretization.

Similar realization questions in the class $\mathrm{A}^{P}$ of Sturm attractors immediately arise when we restrict the class of admissible nonlinearities $f=$ $f\left(x, u, u_{x}\right)$. For example it seems possible to exhaust the class $\mathrm{A}^{P}$ of Sturm attractors by "Hamiltonian" nonlinearities $f=f(x, u)$ alone [Wo02a]. On the other hand, consider nonlinearities with $f(-x, u,-p)=f(x, u, p)$, for all $x, u, p$. Then the reflection symmetry $x \mapsto 1-x$ immediately implies that $\pi_{f}$ is an involution: $\pi_{f}=\pi_{f}^{-1}$. In particular, nonlinearities $f=f(u)$ which depend neither on $x$ nor on $u_{x}$, explicitly, do not generate the class A ${ }^{P}$ completely. Indeed there exist Sturm permutations $\pi_{f}$ with $n=9$ equilibria, which contain 3 -cycles and hence fail to be involutions; see Figure 2.2 below. The minimal such example requires $n=7$ equilibria and is given by $\pi_{f}=(2,4,6)(3,5)$.

Sturm permutations and heteroclinics We address question Q1 next: how to determine all heteroclinics between equilibria? A first answer is given by the following theorem of [FiRo96, Wo02b].

Theorem 2.2.3 The Sturm permutation $\pi=\pi_{f}$, defined by (2.29), (2.30) above, determines explicitly and constructively for all equilibria $U_{j}, U_{k}, 1 \leq$ $j, k \leq n$ of (2.21), (2.22)

(i) the Morse indices (unstable dimensions) $i_{j}, i_{k}$ of $U_{j}, U_{k}$

(ii) the zero numbers $z\left(U_{k}-U_{j}\right)$

(iii) whether or not there exists a heteroclinic solution $u(t)$ from $U_{j}$ to $U_{k}$.

The "explicit and constructive" algorithm asserted by the theorem is slightly involved. For example

$$
i_{k}=\sum_{j=1}^{k-1}(-1)^{j+1} \operatorname{sign}\left(\pi^{-1}(j+1)-\pi^{-1}(j)\right),
$$

and a similar formula holds for the zero numbers $z\left(U_{k}-U_{j}\right)$; see [FiRo96]. Note that indeed $i_{1}=0$ indicates stability of the minimal equilibrium $U^{-}$ as was to be expected from dissipativeness and the monotone convergence to $U^{-}=U_{1}$ mentioned above. Similarly, $i_{n}=0$. In particular, this implies that the total number $n$ of equilibria is odd: just regard the $k-1$ entries \pm 1 in the sum (2.38) which must add up to zero, for $k=n$. Alternatively, this oddness 
can easily be derived from Leray-Schauder degree theory of the equilibria, or from the classical Morse inequalities.

As for assertion (iii) of Theorem 2.2.3, we mention a cascading principle which is peculiar to Sturm attractors. Whenever there is a heteroclinic orbit from $U_{j}$ to $U_{k}$, there exists a cascade of heteroclinic orbits from $U_{j_{0}}=U_{j}$ through $U_{j_{1}}, \cdots, U_{j_{\ell-1}}$ to $U_{j_{\ell}}=U_{k}$ such that the Morse indices drop by 1 along each heteroclinic in the cascade:

$$
i_{j_{\ell}}=i_{j_{\ell+1}}+1 .
$$

By transversality, each heteroclinic orbit in the cascade is just an isolated orbit, locally. It is in fact unique. Already the simple example of a gradient flow with respect to the height function on the standard Euclidean 2-sphere shows that the cascading principle fails for Morse-Smale flows, in general.

Transitivity, in contrast, is a general principle for Morse-Smale flows: if there are heteroclinics from $U_{j_{1}}$ to $U_{j_{2}}$ and from $U_{j_{2}}$ to $U_{j_{3}}$, then there also exists a direct heteroclinic from $U_{j_{1}}$ to $U_{j_{3}}$. The proof is based on the $\lambda$ Lemma.

Combined, cascading and transitivity reduce the problem of finding heteroclinics from $U_{j}$ to $U_{k}$ to the case $i_{j}=i_{k}+1$. Here the zero number and the Sturm property strike again. Such a heteroclinic exists if, and only if, there does not exist an equilibrium $U$, between $U_{j}$ and $U_{k}$ at $x=0$, such that

$$
z\left(U_{j}-U\right) \leq z\left(U_{k}-U\right) .
$$

The "only if" part indeed follows indirectly from Proposition 2.2.1 Just consider

$$
w(t):=u(t)-U
$$

along a hypothetical heteroclinic $u(t)$ from $U_{j}$ to $U_{k}$. Since $U$ lies between $U_{j}$ and $U_{k}$, at $x=0$, and since $w_{x} \equiv 0$ there by the Neumann boundary conditions for $u(t)$ and $U$, a multiple zero of $w$ has to arise at $x=0$, for some $t=t_{0}$. Therefore $z(w(t))$ must drop strictly at least once, in contradiction to (2.40). Thus $U$ as in (2.40) blocks any heteroclinic from $U_{j}$ to $U_{k}$.

The "if" part is proved by a Conley index argument and by a suitable homotopy to a saddle-node bifurcation situation for $U_{j}$ and $U_{k}$. By the explicit formulae for the Morse indices and zero numbers of parts (i) and (ii) of Theorem 2.2.3, this procedure enables us to recursively determine all heteroclinics in the Sturm attractor $\mathcal{A}_{f}$ from the combinatorial ODE information on the Sturm permutation $\pi=\pi_{f}$ alone.

To illustrate Theorems 2.2.2, 2.2.3 we ask for the Sturm attractor $\mathcal{A}$ of maximal dimension $\ell=\max _{k} i_{k}$, with a fixed (odd) number $n$ of equilibria. By our explicit expression (2.38) for the Morse indices $i_{k}$ in terms of the Sturm permutation $\pi$, we first observe $i_{k+1}=i_{k} \pm 1$. Since $i_{0}=i_{n}=0$ are stable, this implies a bound $2 \ell+1 \leq n$. Indeed $i_{k}$ must ascend from $i_{0}=0$ 
to the maximal value $\ell$, and also descend back to $i_{n}=0$. A combinatorial exercise then shows that the maximality requirement $2 \ell+1=n$ for $\ell$ is satisfied if, and only if, the permutation $\pi$ is given by

$$
\pi=\pi_{C I}:=(2, n-1)(4, n-3) \cdots\left(\ell^{\prime}, n+1-\ell^{\prime}\right)
$$

where $\ell^{\prime}$ denotes the largest even integer not exceeding $\ell=(n-1) / 2$. By elementary ODE phase plane methods ("time map", see for example [ChIn74, Sm83, BrCh84, Sc90, BiRo62], the permutation $\pi_{C I}$ arises in the ChafeeInfante problem

$$
f=\mu u\left(1-u^{2}\right) .
$$

Indeed $\pi_{f}=\pi_{C I}$ for $\lambda_{\ell-1}<\mu<\lambda_{\ell}$, where $\lambda_{k}:=(k \pi)^{2}$ and $\ell=\operatorname{dim} \mathcal{A}_{C I}$ is the Morse-index of the trivial equilibrium $U \equiv 0$. The maximal Morse index $\ell$ is indeed attained at $U \equiv 0$, only. By Theorem t-2.2.1, we can now conclude that the Sturm attractor $\mathcal{A}$ of maximal dimension $\ell$ coincides with the Chafee-Infante attractor $\mathcal{A}_{C I}:=\mathcal{A}_{f}$, for $f$ as in (2.43), up to $C^{0}$ orbit equivalence. For an interesting geometric description of the Chafee-Infante attractor see [HaMi91].

Wolfrum has developed a much more elegant approach to Theorem 2.2.3, based on his notions of $z$-ordering and $z$-adjacency. For $z=0,1,2, \cdots$ define partial orders $<_{z}$ on the equilibria $\mathcal{E}=\left\{U_{1}, \cdots, U_{n}\right\}$ as follows: $U_{j}<_{z} U_{k}$ if, and only if,

$$
z\left(U_{j}-U_{k}\right)=z \quad \text { and } \quad U_{j}<U_{k} \quad \text { at } \quad x=0 .
$$

Clearly heteroclinics between such $z$-ordered $U_{j}, U_{k}$ are blocked in the sense of (2.40), by the Sturm property, unless $U_{j}$ and $U_{k}$ are adjacent in this order. Of course $z$-adjacency means that there does not exist another equilibrium $U$ such that $U_{j}<_{z} U<_{z} U_{k}$. We caution the reader that the $z$-order $<_{z}$ is not, in general, transitive.

Wolfrum has proved the following; see [Wo02b].

Theorem 2.2.4 Let $U_{j}, U_{k}$ be distinct equilibria with Morse indices $i_{j}, i_{k}$ in the Sturm attractor $\mathcal{A}_{f}$ of (2.21), (2.22) on the interval. Let $z:=z\left(U_{j}-U_{k}\right)$. Then there exists a heteroclinic orbit from $U_{j}$ to $U_{k}$ if, and only if, $i_{j}>i_{k}$ and $U_{j}, U_{k}$ are $z$-adjacent.

This very concise and direct description of all heteroclinic orbits is again purely combinatorial in terms of the Sturm permutation $\pi=\pi_{f}$. The necessary data $i_{j}, i_{k}, z\left(U_{j}-U_{k}\right)$ are provided by the explicit formulae of parts (i), (ii) of theorem 2.2; see for example (2.38). The result was not, however, derived from that theorem. Instead of cascading, transitivity, and blocking, it is based on a refined geometric analysis of the transversality and zero-number properties along the hierarchies of strong unstable and stable manifolds of all equilibria $U_{j}$. These strong manifolds are submanifolds of $W_{j}^{u}, W_{j}^{s}$ characterized by the faster exponential convergence rates associated to the higher unstable and stable eigenvalues; see for example [BrFi86]. 
Combinatorics of Sturm attractors Theorems 2.2.2 - 2.2.4 highlight the central importance of the Sturm permutation $\pi_{f}$ for the characterization of Sturm attractors $\mathcal{A}_{f}$. It is therefore worthwhile to note that there exists a purely combinatorial characterization of the Sturm permutations, which does not recur to any specific dissipative nonlinearities $f=f\left(x, u, u_{x}\right)$. See [FiRo99] for the following result.

Theorem 2.2.5 Let $\pi \in S_{n}$ be any permutation. Then $\pi$ is a Sturm permutation, that is, $\pi=\pi_{f}$ for some dissipative nonlinearity $f=f\left(x, u, u_{x}\right)$ in (2.21), if, and only if, $n$ is odd and $\pi$ is a dissipative Morse meander.

Here a permutation $\pi \in S_{n}$ is called dissipative, if $\pi(1)=1$ and $\pi(n)=n$. The permutation $\pi$ is called Morse, if $\iota_{k} \geq 0$, holds for all Morse numbers

$$
\iota_{k}:=\sum_{j=1}^{k-1}(-1)^{j+1} \operatorname{sign}\left(\pi^{-1}(j+1)-\pi^{-1}(j)\right),
$$

$k=1, \cdots, n$. Of course, $\iota_{1}:=0$ anyways. To define the meander property of a permutation choose any two distinct numbers $1 \leq j, k \leq n$ of the same even/odd parity, such that $\pi^{-1}(k)$ is between $\pi^{-1}(j)$ and $\pi^{-1}(j+1)$. Then $\pi$ is called a meander permutation, if necessarily $\pi^{-1}(k+1)$ is also between $\pi^{-1}(j)$ and $\pi^{-1}(j+1)$, for any such choice of $j, k$.

We briefly indicate why Sturm permutations $\pi=\pi_{f}$ are necessarily dissipative Morse meanders. The converse direction, which is much more involved, requires the construction of a nonlinearity $f=f\left(x, u, u_{x}\right)$ realizing a prescribed permutation $\pi$ as its Sturm permutation, and will be omitted.

Immediately following our definition (2.29), (2.30) of Sturm permutations $\pi_{f}$, we have already indicated how the monotonicity of the semiflow implies that $\pi_{f}$ is dissipative. The Morse property follows because the Morse numbers $\iota_{k}$ defined in (2.45) coincide with the Morse indices $i_{k}$ of the equilibria $U_{k}$ for Sturm permutations $\pi=\pi_{f}$; see (2.38) and [Ro85, Ro91, FuRo91]. Of course $i_{k} \geq 0$ holds for the Morse indices, which are the dimensions of the unstable manifolds $W_{k}^{u}$.

To understand the meander property we recall Arnold's definition [ArVi89] of a meander permutation. Consider a connected oriented non-selfintersecting curve in the plane, intersecting a fixed oriented base line in $n$ points. The intersections are assumed to be strict crossings. The permutation defined by ordering the intersection points, first along the base line and then along the curve, is called a meander permutation. Here we label the intersection points by $1, \cdots, n$, when ordered along the base line, and by $\pi^{-1}(1), \cdots, \pi^{-1}(n)$ when ordered along the curve. This geometric definition of a meander permutation $\pi$ indeed coincides with ours, by the Jordan curve theorem.

We now show that Sturm permutations $\pi=\pi_{f}$ indeed possess the meander property. We first solve the Neumann boundary-value problem

$$
0:=U_{x x}+f\left(x, U, U_{x}\right)
$$


for equilibria $U=U(x)$ by a "shooting method" in the phase plane $\left(U, U_{x}\right)$. Consider the base line of initial conditions $\left(U, U_{x}\right)=(a, 0), a \in \mathbb{R}$, at $x=0$, given by the $U$-axis. Solving the initial value problem (2.46) up to $x=1$, for this line of initial conditions, we obtain a differentiable curve at $x=1$ in the $\left(U, U_{x}\right)$-plane, which we call the shooting curve. The shooting curve is parameterized by $a$. Intersection points of the shooting curve with the base line $U_{x}=0$ are equilibria: they correspond to the solutions $U_{1}, \cdots, U_{n}$ of the Neumann problem. The labeling by $1, \cdots, n$ along the base line corresponds to the ordering of these solutions at $x=1$. The labeling by $\pi^{-1}(1), \cdots, \pi^{-1}(n)$ along the shooting curve corresponds to the ordering by $a$ at $x=0$. The shooting curve is a connected Jordan curve because the ODE-flow (2.46) from $x=0$ to $x=1$ defines a diffeomorphism of the $\left(U, U_{x}\right)$-plane. Hyperbolicity of the equilibria $U_{1}, \cdots, U_{n}$ corresponds to transverse intersections of the shooting curve with the base line $U_{x}=0$; see [BrCh84, Ro85]. These observations clearly prove that Sturm permutations are indeed dissipative Morse meanders.

With the help of our combinatorial characterization of Sturm permutations it is now possible to characterize Sturm attractors. See Table 2.1 for numbers of Sturm permutations $\pi \in S_{n}, n=1, \cdots, 17$. We note that the trivial transformation $x \mapsto-x$ changes Sturm permutations $\pi$ to their inverse $\pi^{-1}$. Similarly $u \mapsto-u$ conjugates $\pi \in S_{n}$ with the involution $j \mapsto n+1-j$ in $S_{n}$. Neither symmetry operation changes the Sturm attractor, geometrically. See Figure 2.2 for all sixteen Sturm attractors with 9 equilibria together with their Sturm permutations, reduced by the above trivial symmetries [Fi94].

Observe that apparently unrelated Sturm permutations, of different cycle lengths and hence non-conjugate, may lead to the "same" Sturm attractor. Interestingly, these cases differ by the geometry of fast unstable manifolds, as was discovered by Wolfrum [Wo98]; see Figure 2.3. In general, it is still an open question whether this geometric distinction is sufficient to provide a 1-1 correspondence between Sturm attractors $\mathcal{A}_{f}$ and Sturm permutations $\pi_{f}$, up to the above trivial symmetries.

Table 2.1. Numbers of Sturm permutations $\pi=\pi_{f} \in S_{n}$ for odd $n=1, \cdots, 17$

\begin{tabular}{|r|c|c|c|}
\hline $\mathrm{n}$ & dissipative meanders & Sturm permutations & Sturm mod symmetry \\
\hline \hline 1 & 1 & 1 & 1 \\
\hline 3 & 1 & 1 & 1 \\
\hline 5 & 2 & 2 & 2 \\
\hline 7 & 8 & 7 & 5 \\
\hline 9 & 42 & 32 & 18 \\
\hline 11 & 262 & 175 & 75 \\
\hline 13 & 1828 & 1083 & 383 \\
\hline 15 & 13820 & 7342 & 2850 \\
\hline 17 & 110954 & 53372 & 14984 \\
\hline
\end{tabular}



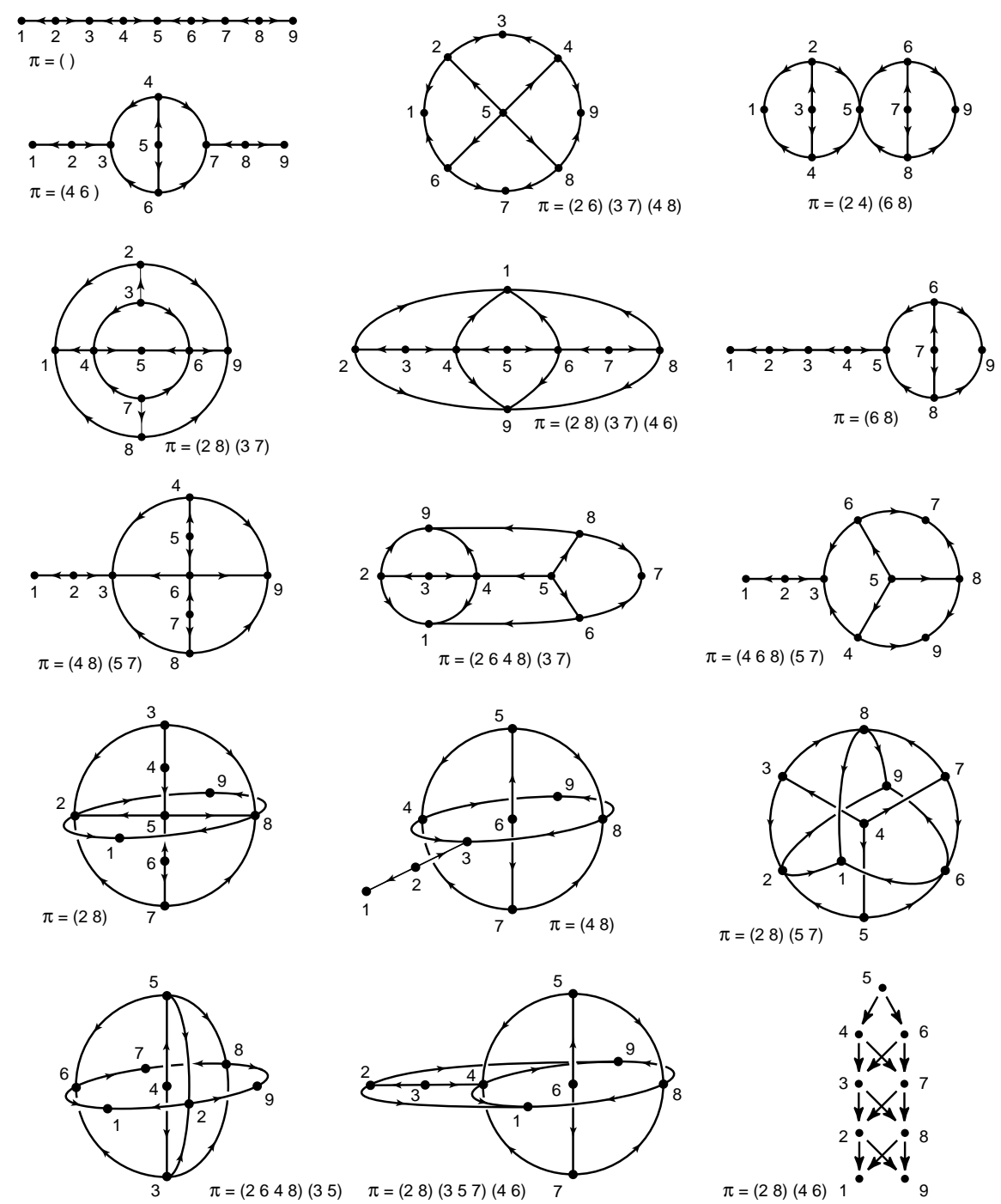

Fig. 2.2. All sixteen Sturm attractors $\mathcal{A}_{f}$ with $n=9$ equilibria. The corresponding Sturm permutations $\pi_{f}$ are indicated in cycle notation.

We conclude this section with a short summary of the intriguing results by Härterich on the Sturm attractors $\mathcal{A}_{\varepsilon}$ of viscous approximations to nonlinear hyperbolic balance laws; see [Hä98]. For scalar equations in one space dimension such viscous approximations take the form

$$
u_{t}+F(u)_{x}+G(u)=\varepsilon u_{x x},
$$



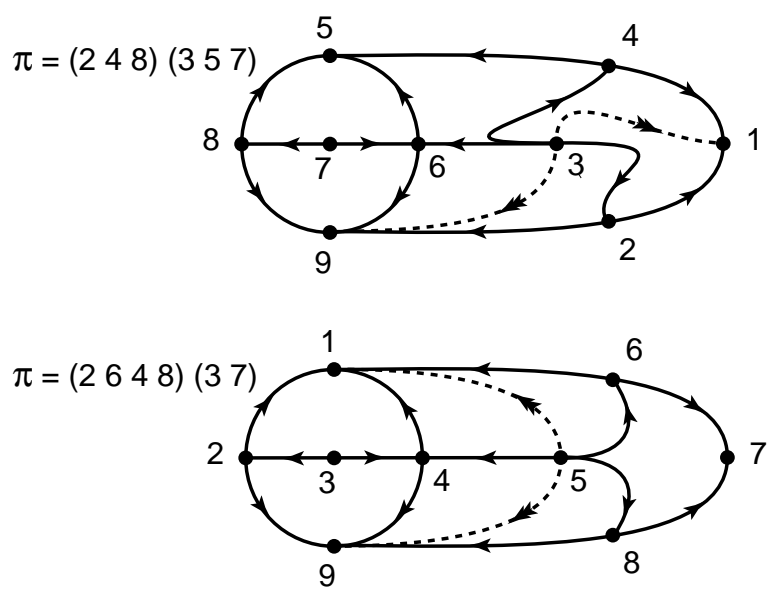

Fig. 2.3. $C^{0}$ orbit equivalent Sturm attractors $\mathcal{A}_{f}$ with nonconjugate permutations $\pi_{f}$. Note how the attractors differ in the geometry of the fast unstable manifolds, indicated by double arrows.

This fits in the framework (2.21), (2.22) with $f:=F^{\prime}(u) u_{x}+G(u)$, under Neumann boundary conditions on the unit interval. The object of interest is the limiting behavior of the Sturm attractor $\mathcal{A}_{\varepsilon}$, for vanishing viscosity $\varepsilon \searrow 0$. Härterich has derived sufficient conditions on $F, G$ such that

(i) $\ell_{\varepsilon}:=\operatorname{dim} \mathcal{A}_{\varepsilon}$

(ii) $n_{\varepsilon}:=$ the number of equilibria of $\mathcal{A}_{\varepsilon}$

(iii) $\pi_{\varepsilon}:=$ the Sturm permutations of $\mathcal{A}_{\varepsilon}$

(iv) $\mathcal{A}_{\varepsilon}$ up to orbit equivalence

all stabilize, that is, eventually become independent of $\varepsilon$ for $0<\varepsilon<\varepsilon_{0}$. In view of the Chafee-Infante example (2.43), which is equivalent to (2.47) with $F=0$, cubic $G$, and $\varepsilon:=1 / \mu$, this is surprising. Indeed $\ell_{\varepsilon}=1+\left[(\pi \sqrt{\varepsilon})^{-1}\right]$ and $n_{\varepsilon}=2 \ell_{\varepsilon}+1$ grow unboundedly for $\varepsilon \searrow 0$, in this case.

In [Hä98] sufficient conditions for a uniform dimension bound $\ell_{\varepsilon} \leq \ell_{0}$ were derived, for $\varepsilon \searrow 0$. Specifically, if $F$ possesses only nondegenerate critical points and $G$ does not vanish at any critical point of $F$, then there exist numbers $\ell_{0} \in \mathbb{N}$ and $\varepsilon_{0}>0$ such that $\ell_{\varepsilon} \leq \ell_{0}$ for all $0<\varepsilon \leq \varepsilon_{0}$.

In [Hä97] these conditions were refined to imply stabilization of $\pi_{\varepsilon}$, (iii). Note that (iii) implies stabilization (ii) of $n_{\varepsilon}$, trivially, and stabilization (i) of $\ell_{\varepsilon}=\operatorname{dim} \mathcal{A}_{\varepsilon}$, by our explicit representation (2.38) for the Morse indices $i_{k}$. Specifically, there exists an open class of functions $F$ and $G$ (with respect to the strong Whitney topology) such that the following holds: There exist numbers $\ell_{0} \in \mathbb{N}$ and $\varepsilon_{0}>0$ such that $\ell_{\varepsilon} \in\left\{\ell_{0}-1, \ell_{0}\right\}$ for all $0<\varepsilon \leq \varepsilon_{0}$. Explicit conditions on $F$ and $G$ can also be stated which can be verified in concrete situations. Note that it is not clear whether $n_{\varepsilon}$ stabilizes for $\varepsilon \rightarrow 0$. 
If $F \in C^{2}$ is strictly convex and $G \in C^{1}$ possesses only simple zeros that do not coincide with the critical point of $F$, then all four stabilization properties (i)-(iv) hold, as was claimed above; see [Hä99].

The proofs of these results are based on a very careful singular perturbation analysis of the ODE equilibrium problem, for $\varepsilon \searrow 0$, including solutions of canard type.

For another example of a singular perturbation of Sturm permutations and Sturm attractors, involving $x$-dependent nonlinearities $f=f(x, u)$ of cubic Chafee-Infante type in $u$, see [Fi\&al02a] and the references there.

\subsubsection{Sturm Attractors on the Circle}

In Section 2.2.1, (2.8)-(2.10) we have seen how the case $x \in S^{1}=\mathbb{R} / \mathbb{Z}$ of periodic boundary conditions for the scalar reaction-advection-diffusion equation

$$
u_{t}=u_{x x}+f\left(x, u, u_{x}\right)
$$

is a hybrid between one- and two-dimensional domains $\Omega$. The gradient-flow property fails, due to rotating waves which arise in the $S^{1}$-equivariant case $f=f\left(u, u_{x}\right)$. The Sturm property on the other hand prevails; see Proposition 2.2.1. At the end of Section 2.2.1 we have used the Sturm property, only, to prove a gradient-flow feature of (2.48) on the interval: convergence to equilibrium for any bounded solution. We will first indicate the fate of this convergence property on the circle domain. We will then present an analogue of Theorems 2.2.3, 2.2.4 for heteroclinic orbits between rotating waves.

Poincaré-Bendixson theory Intuitively we can say that the dynamics of $(2.48)$ is "essentially one-dimensional", asymptotically for any single trajectory $u(t)=\mathcal{T}(t) u_{0}$ and under separated boundary conditions. Indeed, $u(t)$ converges to a single equilibrium as would be the case for any scalar ODE $\dot{\mathbf{u}}=g(\mathbf{u}), \mathbf{u} \in \mathbb{R}$. Still the global attractor $\mathcal{A}_{f}$ may be of arbitrarily large dimension $\ell$, as we recall from the Chafee-Infante example (2.43).

The dynamics of (2.48) on the circle $x \in S^{1}$ is then "essentially twodimensional", asymptotically for $u(t)=\mathcal{T}(t) u_{0}$. The analogous convergence statement is given by the Poincaré-Bendixson theorem, well known from ODE dynamics $\dot{\mathbf{u}}=g(\mathbf{u})$ in the phase plane $\mathbf{u} \in \mathbb{R}^{2}$. Of course, the Chafee-Infante example persists under periodic boundary conditions, if we reflect solutions through their Neumann boundary. Therefore, the global attractor may still be of arbitrarily large dimension.

For a precise statement of our Poincaré-Bendixson result we fix any $x_{0} \in$ $S^{1}$, arbitrarily, and consider the evaluation projection

$$
\begin{aligned}
P: X & \rightarrow \mathbb{R}^{2} \\
u & \mapsto\left(u\left(x_{0}\right), u_{x}\left(x_{0}\right)\right) .
\end{aligned}
$$


The following theorem has been proved in [FiMP89b]; for a related result obtained independently see [Na90]. Recall that $\mathcal{E}=\left\{u_{t}=0\right\}$ denotes the set of equilibria.

Theorem 2.2.6 Fix any $x_{0} \in C^{1}$. Let $u_{0} \in X \hookrightarrow C^{1}$ possess a uniformly bounded solution $u(t)=\mathcal{T}(t) u_{0} \in X, t \geq 0$, of (2.48) with $\omega$-limit set $\omega\left(u_{0}\right)$.

Then the evaluation projection $P: \omega\left(u_{0}\right) \rightarrow \mathbb{R}^{2}$ is injective on the $\omega$-limit set $\omega\left(u_{0}\right)$. Moreover the following alternative holds:

(i) either $\omega\left(u_{0}\right)$ is a single periodic orbit, or else

(ii) both the $\alpha$-limit set and the $\omega$-limit set of any nonequilibrium solution $\tilde{u}(t), t \in \mathbb{R}$, in $\omega\left(u_{0}\right)$ consist entirely of equilibria.

To indicate the relation of Theorem 2.2.6 with the Sturm property, Proposition 2.2.1, we show injectivity of the restricted evaluation projection

$$
P: \operatorname{clos}\left(\operatorname{orb}\left(\tilde{u}_{0}\right)\right) \rightarrow \mathbb{R}^{2}
$$

only along the $X$-closure of any single trajectory $\operatorname{orb}\left(\tilde{u}_{0}\right):=\left\{\mathcal{T}(t) \tilde{u}_{0} ; t \in\right.$ $\mathbb{R}\}$ within the original $\omega$-limit set: $\tilde{u}_{0} \in \omega\left(u_{0}\right)$. Invoking the Jordan curve theorem, it is then easy to show that $\operatorname{clos}\left(\operatorname{orb}\left(\tilde{u}_{0}\right)\right)$ contains a periodic orbit, if it does not contain any equilibrium. See for example [Fi89]. The fine tuning which leads to Theorem 2.2.6 is much more involved, see [FiMP89b].

To prove restricted injectivity (2.50), choose any two distinct initial conditions $\tilde{u}_{0}^{1}, \tilde{u}_{0}^{2}$ in $\operatorname{clos}\left(\operatorname{orb}\left(\tilde{u}_{0}\right)\right)$. Suppose that $P \tilde{u}_{0}^{1}=P \tilde{u}_{0}^{2}$, proceeding indirectly. Denoting solution curves through $\tilde{u}_{0}^{\iota}, \iota=1,2$, by $\tilde{u}^{\iota}(t), t \in \mathbb{R}$, we then see that

$$
t \mapsto z\left(\tilde{u}^{1}(t)-\tilde{u}^{2}(t)\right)
$$

drops strictly at $t=0$, by Sturm proposition 2.2.1(ii). Choosing $t_{0}$ and $t_{0}+\vartheta$ large enough, (2.51) implies that

$$
t \mapsto z\left(\tilde{u}\left(t+t_{0}+\vartheta\right)-\tilde{u}\left(t+t_{0}\right)\right)
$$

also drops, for some $|t| \leq 1$. Indeed, the $x$-profile $\tilde{u}^{1}(t)-\tilde{u}^{2}(t)$ possesses only simple zeros in $x$ before and after dropping, and $\tilde{u}_{0}^{1}, \tilde{u}_{0}^{2} \in \operatorname{clos}\left(\operatorname{orb}\left(\tilde{u}_{0}\right)\right)$ in the topology of $X \subseteq C^{1}$. Since $\tilde{u}_{0} \in \omega\left(u_{0}\right)$, the dropping in (2.52) implies that there also exists an increasing sequence $t_{n} \nearrow \infty$ of dropping times $t=t_{n}$ of

$$
t \mapsto z\left(u\left(t+t_{0}+\vartheta\right)-u\left(t+t_{0}\right)\right),
$$

for the original solution $u$. This contradicts the finiteness property (iii) of the Sturm Proposition2.2.1, and hence proves our injectivity claim (2.50) indirectly.

Similar Poincaré-Bendixson theorems have been proved to hold for differential delay equations 


$$
\dot{u}(t)=f(u(t), u(t-1))
$$

under a positive or negative monotonicity assumption on $f$ in the delayed feedback argument $u(t-1)$; see [MP88, MPSm90]. For discrete analogues, the so-called cyclic monotone feedback systems, see [Sm95] as well as [MPSe96b] for combinations of both structures. The approach in [FiMP89b] is axiomatic, and includes Jacobi systems (2.36).

It is interesting to note that (2.54) under positive feedback, where $f(u, \cdot)$ is strictly increasing, does not exhibit asymptotically stable periodic solutions. This is reminiscent of the Hirsch theorem on the absence of stable periodic orbits for monotone dynamical systems, as discussed in Section 2.2.1. For negative feedback, in contrast, stable periodic solutions of (2.54) do arise; see for example [Di\&al95] and the references there. As a curiosity, we note here the twisted periodic boundary condition

$$
\begin{aligned}
u(t, 1) & =-u(t, 0) \\
u_{x}(t, 1) & =-u_{x}(t, 0)
\end{aligned}
$$

for which all the above results remain valid, due to the Sturm structure. The monotonicity property fails, however: although formally in effect, it is prevented by the twisted boundary condition (2.55). Indeed $z(\tilde{u}-u) \geq 1$ for any two distinct solution profiles $\tilde{u}, u$ satisfying (2.55), as soon as $t>0$. This phenomenon is closely related to the Smale objection to monotonicity, indicated in Section 2.2.1. Explicit examples of odd rotating wave solutions $u=u(x-c t)$ for suitable $f$ show that asymptotically stable periodic orbits in fact do occur under the twisted boundary condition (2.55). Unfortunately this is a purely mathematical observation, at the moment: we are not aware of any applied relevance of our twisted periodic boundary condition.

Heteroclinic connections of rotating waves When we now attempt to describe the Sturm attractors $\mathcal{A}_{f}$ under the usual periodic boundary conditions $x \in S^{1}=\mathbb{R} / \mathbb{Z}$, the Poincaré-Bendixson Theorem 2.2.6 encourages us to pursue the program outlined in questions $\mathbf{Q 0}-\mathbf{Q} 4$ of Section 2.2.2. Of course we will have to replace the word "equilibria" in Q0, Q1 and address "equilibria and time periodic orbits" as well as their heteroclinic orbits, instead. A fundamental obstacle, however, arises immediately: the loss of the Morse-Smale transversality property. In fact, nontransverse intersections of stable and unstable manifolds may arise. Specifically, any autonomous planar ODE vector field can be embedded into (2.48) for a suitable choice of the nonlinearity $f=f\left(x, u, u_{x}\right), x \in S^{1}$; see [SaFi92] and [Br\&al92]. The dynamics is supported, for example, on a time invariant linear subspace spanned by the spatial Fourier modes $\sin x$ and $\cos x$. Embedding any planar homoclinic orbit then immediately shows that the Morse-Smale property may fail, even when all equilibria and periodic orbits are hyperbolic. As an aside we mention that time periodic nonlinearities $f=f\left(t, x, u, u_{x}\right), x \in S^{1}$, can give rise to 
transverse homoclinic orbits and hence to Smale horseshoes with Bernoulli type shift dynamics, by similar constructions; see [SaFi92].

A second difficulty of periodic boundary conditions lies in the combinatorial description of $\mathcal{A}_{f}$. The Fusco-Rocha definition (2.29), (2.30) of the Sturm permutation $\pi_{f}$, by ordering of the boundary values $u$, clearly depends on the separated nature of the boundary conditions at $x=0,1$. The Sturm permutation probably would have to be replaced by the braid type of the braid in $\left(x, u, u_{x}\right) \in S^{1} \times \mathbb{R}^{2}$, which is defined by the $x$-profiles of all equilibria and time periodic orbits. Any periodic orbit $u(t)$ in $X$ can in fact be represented by any snap-shot $u\left(t_{0}\right)$ without changing the braid type. In the case of Neumann boundary conditions the braid embeds into a surface: the shooting surface of solution of (2.46) with $U_{x}(0)=0$. The braid type is then in fact determined by the Sturm permutation $\pi=\pi_{f}$.

The above remarks sufficiently demonstrate our lack of understanding of the general case $f=f\left(x, u, u_{x}\right)$. We therefore now restrict to the $S^{1}$ equivariant case of $x$-independent nonlinearities

$$
u_{t}=u_{x x}+f\left(u, u_{x}\right), \quad x \in S^{1} .
$$

From Section $2.2 .1,(2.9),(2.10)$ we recall that rotating waves $u(t, x)=U(x-$ $c t)$ arise in that case. More precisely it was proved in [AnFi88] that all periodic orbits $U(t, x)$ are rotating waves $U=U(x-c t)$. This follows from Sturm Proposition 2.2.1: the zero number $z$ must be constant on the span of $U_{t}, U_{x}$, and hence $U_{t}$ and $U_{x}$ must indeed be linearly dependent. The Sturm attractor $\mathcal{A}_{f}$ then consists of equilibria $\mathcal{E}$, rotating waves $\mathcal{R}$, and their heteroclinic orbits $\mathcal{H}$ :

$$
\mathcal{A}_{f}=\mathcal{E} \cup \mathcal{R} \cup \mathcal{H}
$$

We assume $\mathcal{E}$ and $\mathcal{R}$ to be normally hyperbolic, admitting at most a single trivial eigenvalue (or Floquet exponent) resulting from $x$-shift.

To formulate our result on heteroclinic connections in $\mathcal{A}_{f}$, we slightly adapt Wolfrum's notation of $z$-adjacency, from (2.44). First we represent any nonhomogeneous equilibrium in $\mathcal{E}$ and any rotating wave in $\mathcal{R}$ by two elements $U \in X$ satisfying $U_{x}=0$ at $x=0$. This can always be achieved because $\mathcal{E}, \mathcal{R}$ are invariant under $x$-shift, by $S^{1}$-equivariance of (2.56). The two representatives are chosen to attain their maximum and their minimum at $x=0$, respectively. We then define $z$-order and $z$-adjacency as in (2.44), based on the ordering and adjacency properties of these representatives, at $x=0$.

We also adapt the Morse index $i$ of $U \in \mathcal{E} \cup \mathcal{R}$ to indicate the strong unstable dimension. For equilibria, $i$ counts the total algebraic multiplicity of complex eigenvalues $\lambda$, after linearization, with strictly positive real part. For rotating waves $U$ we similarly count the nontrivial Floquet multipliers $\lambda$ strictly outside the unit circle.

The following theorem is due to [Fi\&al02b] and assumes normal hyperbolicity; see (2.57). 
Theorem 2.2.7 Let $U_{j}, U_{k}$ be equilibria or rotating waves with strong unstable dimensions $i_{j}, i_{k}$ in the Sturm attractor of the $S^{1}$-equivariant reactionadvection-diffusion equation (2.56) on the circle $x \in S^{1}$. Let $z:=z\left(U_{j}-U_{k}\right)$. Then there exists a heteroclinic orbit from $U_{j}$ to $U_{k}$ if, and only if, $i_{j}>i_{k}$ and $U_{j}$ and $U_{k}$ are $z$-adjacent.

The proof of this theorem at present involves four steps, which we call

(i) transversality

(ii) freezing

(iii) symmetrization

(iv) Neumann embedding

We only briefly mention the topics involved. In step (i), we slightly extend results by [O102] to show that the Morse-Smale property of transverse intersections of unstable and stable manifolds holds, in the $S^{1}$-equivariant case, for normally hyperbolic equilibria and rotating waves. This allows us, in step (ii), to construct a homotopy $f^{\tau}$ of $f=f^{0}$ which "freezes" all rotating waves:

$$
f^{\tau}:=f\left(u, u_{x}\right)+\tau c u_{x}
$$

Here $c=c\left(u, u_{x}\right)$ is chosen to coincide with the constant wave speed along the $x$-profile $\left(u, u_{x}\right)(x)$ of any rotating wave of $f$, without introducing any additional rotating waves for any $0 \leq \tau \leq 1$. In effect, this homotopy reduces the speed $c_{0}$ of any rotating wave at $\tau=0$ to

$$
c_{\tau}=(1-\tau) c_{0}
$$

By $\tau=1$, any rotating wave speed has been tuned down to zero, and all periodic orbits have become spatially nonhomogeneous equilibria: $\mathcal{R}=\emptyset$ in (2.57). Step (iii) performs a second homotopy which keeps all (frozen) equilibria normally hyperbolic and reduces $f=f\left(u, u_{x}\right)$ to a nonlinearity which is even in $u_{x}$ and hence commutes with the reflection $x \mapsto 1-x$. In particular, any equilibrium solution $U$ becomes reflection symmetric with respect to any of its local maxima or minima, by this $x$-reversibility. Shifting any equilibrium $U \in \mathcal{E}$ by some suitable value $x_{0}$, we may therefore assume $U_{x}=0$ at $x=0$ and $x=1 / 2$. In step (iv) we observe that all heteroclinic orbits of Theorem 2.2.4 are then in fact already represented by reflection symmetric heteroclinic orbits. The latter are already known: we only have to apply Wolfrum's Theorem 2.2.4. to the resulting Neumann problem on the half-interval $0 \leq x \leq 1 / 2$. This completes our sketch of a proof of Theorem 2.2.7. For complete details see [Fi\&al02b].

We conclude this section with a few remarks concerning related results. The results by Hale and Raugel concerning upper and lower semicontinuity of attractors $\mathcal{A}_{f}$ for thin domains [HaRa92] allow us to fatten the circle $\Omega_{0}=S^{1}$ to thin annuli $\Omega_{\varepsilon}=S^{1} \times(-\varepsilon, \varepsilon)$ and recover all dynamics of $\mathcal{A}_{f}$ on $\Omega_{\varepsilon}$. More 
generally domains $\Omega_{\varepsilon}=S^{1} \times \tilde{\Omega}_{\varepsilon}$ work, for suitably "small" cross sections $\tilde{\Omega}_{\varepsilon}$ in terms of a sufficiently large second eigenvalue of the Neumann problem on the cross section $\tilde{\Omega}_{\varepsilon}$. Similarly, nonlinearities $f+\varepsilon \tilde{f}$ can be allowed to depend on $x$, slightly, via $\tilde{f}\left(x, u, u_{x}\right)$ and small $\varepsilon$. In the spirit of [FiVi01a, FiVi01b], nonlinearities $f=f\left(\varepsilon, x / \varepsilon, u, u_{x}\right)$ are also admissible, under Diophantine quasiperiodicity conditions on the rapid spatial dependence $x / \varepsilon$ of $f$.

Fast travelling waves in cylinder domains $x=(\xi, \eta) \in \Omega=S^{1} \times \mathbb{R}$ provide another interpretation of our heteroclinic orbits. Consider travelling wave solutions

$$
u(t, x)=U(t-\varepsilon \eta, x)
$$

of a scalar $x$-independent parabolic equation (2.1). For large wave speeds $1 / \varepsilon$ in the unbounded $\eta$-direction, we obtain

$$
U_{\tau}=U_{\xi \xi}+\varepsilon^{2} U_{\tau \tau}+f\left(U, U_{\xi},-\varepsilon U_{\tau}\right),
$$

abbreviating $\tau:=t-\varepsilon \eta$. In [Sc96] it was shown how to omit both $\varepsilon$-terms, in the fast wave speed limit $\varepsilon \searrow 0$. See also [Ca\&al93] for nonlinearities $f$ which do not depend on the gradient term $U_{\xi}$. Invoking Theorem 2.2.7 then provides heteroclinic orbits $U(\tau, \xi)$ between rotating waves $U_{1}\left(\xi-c_{1} \tau\right), U_{2}\left(\xi-c_{2} \tau\right)$, typically rotating at different angular speeds $c_{1}, c_{2}$. Clearly $U(\tau, \xi)$ then describes a nonmonotone wave $u$ propagating rapidly along the cylinder axis $\eta$ of $\Omega$, and connecting $\eta$-asymptotic states which rotate at different angular $\xi$ speeds $c_{1}, c_{2}$. The complexity of such wave profiles is in marked contrast with the monotone travelling waves found by Berestycki and Nirenberg using comparison methods [BeNi90]. See also the beautiful complementary approach by a variational characterization in [He89].

Returning to one-dimensional $x$ we conclude this section by indicating why results like Theorems 2.2.2 - 2.2.7 do not carry over to systems $u \in$ $\mathbb{R}^{N}, N=2$, not even in a single space dimension. Following [FiPo90], we consider

$$
u_{t}=u_{x x}+f(x, u)+c(x) \int_{0}^{1} u \mathrm{~d} x,
$$

only for scalar $u \in \mathbb{R}$, but involving a linear nonlocal term $c(x) \int u d x$. We consider $0<x<1$ with Dirichlet boundary conditions. It has then been proved that very general finite-dimensional vector fields $\dot{\mathbf{u}}=g(\mathbf{u}), \mathbf{u} \in \mathbb{R}^{\mathbf{n}}$ arise, on an invariant (center) manifold of (2.61) and up to any finite polynomial order, for suitable choices of $f, c$. The constraints on $g(\mathbf{u})$ are rather mild: $g(0)=0$ and the eigenvalues of $g^{\prime}(0)$ on the imaginary axis should be simple. In particular this includes the possibility of complicated dynamics of Bernoulli shift type. These results are very similar, in spirit, to earlier analogous observations by Hale for retarded functional differential equations; see [Ha85]. 
Similar remarks apply to the case of scalar equations (2.1), $u \in \mathbb{R}^{N}, N=$ 1 , in several space dimensions. Dancer and Poláčik [DaPo02] have proved that a $C^{1}$-dense set of finite-dimensional vector fields $\dot{\mathbf{u}}=g(\mathbf{u}), \mathbf{u} \in$ $\mathbb{R}^{\mathbf{n}}$ can be realized on an invariant manifold, for suitable choices of $x$ independent reaction-advection nonlinearities $f=f(u, \nabla u)$ and of twodimensional smooth bounded domains $\Omega \subset \mathbb{R}^{2}$. For suitable $f=f(x, u, \nabla u)$ with only linear dependence on $\nabla u$ and arbitrary, but fixed domains $\Omega \subset \mathbb{R}^{2}$, a similar result is due to [Po95], [PrRy98a, PrRy98b]. The gradient-like case $f=f(x, u)$ on a two-dimensional ball allows embeddings of $C^{1}$-dense sets of gradient vector fields $g(\mathbf{u})=-\nabla_{\mathbf{u}} G(\mathbf{u})$. See also the survey [Po02].

These results certainly motivate a quest for more refined structural conditions on nonlinearities $f$, as well as domains $\Omega$, which impose incisive restrictions on their resulting spatio-temporal dynamics.

\subsection{One Unbounded Space-Dimension: Travelling Waves}

In this chapter, we address some aspects of the dynamics of reaction-diffusion systems in large or unbounded, one-dimensional domains. Motivated by the motion of layers and fronts, we study essential spectra that arise in the linearization about travelling wave solutions, Section 2.3.1. In Section 2.3.2, we extend this linear analysis to a nonlinear bifurcation result. Depending on Fredholm indices in the essential spectrum, we find existence or nonexistence of periodic orbits in a Hopf bifurcation caused by the essential spectrum. We conclude this chapter with an investigation of the limiting behavior of the spectrum of the linearization about a travelling wave, when the domain size tends to infinity. Under separated boundary conditions, the limiting spectrum differs from the spectrum in the unbounded domain. The continuous parts of the limiting spectrum consist of a finite collection of curves which we call the absolute spectrum.

\subsubsection{Unbounded Domains and Essential Spectra}

From bounded to unbounded domains The results on global attractors in scalar reaction-diffusion equations reviewed in the preceding sections show that certain dynamical properties do not depend on boundary conditions. The Sturm nodal property prevails, for example, independently of the boundary conditions. This allows for homotopies between Neumann, Dirichlet, mixed, and sometimes even periodic boundary conditions. An extreme statement in this direction is that the class - in contrast to individual global attractors - of all Sturm attractors $\mathrm{A}^{P}$ does not depend on the type of (separated) boundary conditions; see Section 2.2.2.

In descriptions of phenomena in experiments, it is often quite desirable to separate the influence of the boundary from what we call the inner dynamics of a reaction-diffusion system. See for example [Bl\&al00] for a critical view on 
the influence of boundary conditions in chemical experiments. Mathematical statements on the influence of boundaries can be found mainly in the context of singular perturbation theory. For an illustration, we rewrite the ChafeeInfante problem (2.43) as

$$
u_{t}=\varepsilon^{2} u_{x x}+u(1-u)(1+u), \quad x \in(0,1)
$$

say with Neumann boundary conditions. Rescaling $x$, the parameter $\varepsilon$ can be interpreted as a measure for the size of the domain, $0 \leq x \leq L=1 / \varepsilon$. For small $\varepsilon$, the motion on the unstable manifolds of equilibria (which constitute the Chafee-Infante attractor, by (2.27)) becomes exponentially slow in the parameter $\varepsilon$; see [FuHa89, CaPe90]. "Most" of the time, solutions consist of a finite collection of transition layers at $x=x_{j}(t)$ of the approximate local form

$$
u_{j} \sim \pm \tanh \left(\frac{x-x_{j}(t)}{\sqrt{2} \varepsilon}\right) .
$$

The exponentially slow motion $x_{j}(t)$ is driven by the inner dynamics, caused by interaction and annihilation of layers, and the influence of the boundary. The strength of the interaction and the influence of the boundary indeed decrease exponentially with distance:

$$
\left|\dot{x}_{j}(t)\right| \leq C \exp \left(-C^{\prime}\left(\inf _{j^{\prime} \neq j}\left\{\left|x_{j}-x_{j^{\prime}}\right| / \varepsilon\right\}+\min \left\{\left|x_{j}\right|,\left|1-x_{j}\right|\right\}\right)\right) .
$$

For most initial conditions, the slow motion eventually leads to annihilation of all layers and we recover the Hirsch result [Hi88] on generic convergence to stable equilibria in monotone dynamical systems, $u_{ \pm}(x) \equiv \pm 1$ in our case however only after time spans which may well exceed realistic experimental conditions.

The limiting case $\varepsilon=0$ is best described in the scaling

$$
u_{t}=u_{x x}+u(1-u)(1+u), \quad x \in(-L, L),
$$

with $L=1 / \varepsilon$. In the limit $L=\infty$, we are led to consider the unbounded real line as an idealization describing the inner dynamics.

One important but largely unresolved question concerning the limit $L \rightarrow \infty$ of increasing domain size, is in how far energy considerations can be localized, separating effects of the boundary from the intrinsic dynamics. Energy, alias the Lyapunov function in (2.4), is a nonlocal function and need not even be finite in an unbounded domain. Gallay and Slijepčevič [GaSl02] show how this fact may lead to recurrent, quite unexpected behavior in "gradient" systems.

Unbounded domains feature yet another phenomenon: non-compact symmetry. Reaction-diffusion systems (2.1) with $x$-independent reaction term $f=f(u, \nabla u)$, are invariant under spatial translation $x \rightarrow x+g$ : if $u(t, x)$ is a solution, so is $u(t, x+g)$ for any $g \in \mathbb{R}$, fixed. The slow motion $x_{j}(t)$ of layers in the Chafee-Infante problem reflects this translational symmetry. The time 
evolution is "composed" of translates of the single layer (2.63). Whereas the role of the noncompact symmetry group of translations $g \in \mathbb{R}$ is obvious, here, Euclidean symmetry $G=S E(2)$ will play a major and more subtle role in the discussion of spiral wave dynamics in two-dimensional domains; see Chapter 2.5.

Separated boundary conditions break this translational equivariance. Periodic boundary conditions, in contrast, preserve the action of at least a continuous, compact group $S O(2)=\mathbb{R} / \mathbb{Z}$ as a remaining symmetry, when the real line is truncated to a bounded interval; see Section 2.2.3. Still, even periodic boundary conditions fail to mimic the translational drift along noncompact group orbits like the observed motion of a single stable front: under periodic boundary conditions, fronts come in pairs and typically annihilate or strongly interact after some finite time interval. We will further investigate the role of boundary conditions in the presence of transport and drift in Section 2.3.3.

If the domain is the real line $x \in \mathbb{R}$, the discussion of equilibria in $u_{t}=$ $u_{x x}+f\left(u, u_{x}\right)$ has at least to be augmented to include travelling waves, $u(t, x)=q(x-c t), c \in \mathbb{R}$, with $\sup _{\xi} u(\xi)<\infty$, just like in the case of periodic boundary conditions, Section 2.2.3. Travelling waves are a special case of relative equilibria, where time evolution of a profile is described by motion along the group action, here, translated states. We discuss relative equilibria more generally in the context of spiral wave dynamics in Chapter 2.5. Fronts are special travelling waves, where $q(\xi)$ possesses a heteroclinic asymptotic behavior for $\xi \rightarrow \pm \infty$, for example $q(\xi) \rightarrow q_{ \pm}$for $\xi \rightarrow \pm \infty$. A particular case are the layers in the Chafee-Infante problem, where $c=0$.

Spectra of travelling waves: group velocities and Fredholm indices For a scalar equation $u_{t}=u_{x x}+f(u)$, travelling waves with $c \neq 0$ are either spatially constant, $u(\xi) \equiv u_{0}$, or heteroclinic front solution. This is due to the gradient-like structure of the travelling-wave ordinary differential equation

$$
u_{\xi}=v, \quad v_{\xi}=-c v-f(u) .
$$

More generally, we address reaction-diffusion systems

$$
u_{t}=D u_{x x}+f(u),
$$

with $x \in \mathbb{R}, u \in \mathbb{R}^{N}$ and a positive, diagonal diffusion matrix $D=\operatorname{diag}\left(d_{j}\right)>$ 0 . Systems of several reaction species, $N>1$, in general, neither possess gradient-like structure, nor monotonicity properties - or even nodal properties - in the sense of Section 2.2.1. Still, heteroclinic travelling-wave solutions $u(t, x)=q_{*}(x-c t)$ remain a fundamental, and elementary, ingredient to their dynamics.

Given such a travelling wave, we ask for its stability. We therefore consider the reaction-diffusion system in a comoving frame $\xi=x-c t$,

$$
u_{t}=D u_{\xi \xi}+c u_{\xi}+f(u), \quad x \in \mathbb{R}
$$


where the travelling wave becomes an equilibrium $q_{*}(\xi)$. Throughout, $x$ always refers to the spatial coordinate in the steady frame, whereas $\xi$ always refers to a comoving frame with speed $c \geq 0$. The case $c<0$ is obtained reflecting $x \rightarrow-x$. The travelling wave moves towards $x=+\infty$ and, in the comoving frame, the reaction-diffusion system inherits a drift term $c u_{\xi}$, representing transport towards $x=-\infty$. In the comoving frame, we linearize at $q_{*}$ to find

$$
w_{t}=D w_{\xi \xi}+c w_{\xi}+f^{\prime}\left(q_{*}(\xi)\right) w=: \mathcal{L}_{*} w .
$$

For simplicity, we first consider the operator $\mathcal{L}_{*}$ as a closed, unbounded operator on $L^{2}(\mathbb{R})$. All results of this section remain valid if we replace the function space $L^{2}$ by $L^{p}$, or by $1<p<\infty$, or bounded, uniformly continuous functions. The spectrum spec $\mathcal{L}_{*} \subset \mathbb{C}$ is defined as the set of $\lambda$ such that $\mathcal{L}_{*}-\lambda$ is not boundedly invertible. Various notions of stability for individual fronts $q_{*}$ have been suggested. We call a travelling wave spectrally stable, if the spectrum of the linearized operator $\mathcal{L}_{*}$ is contained in $\{\operatorname{Re} \lambda \leq 0\}$. We caution the reader that spectral stability need not imply asymptotic stability on our unbounded domain $x \in \mathbb{R}$.

For a refined discussion of stability properties, we decompose the spectrum into the essential spectrum

$$
\operatorname{spec}_{\text {ess }} \mathcal{L}_{*}:=\left\{\lambda \in \mathbb{C} ; \mathcal{L}_{*}-\lambda \text { is not Fredholm of index } 0\right\}
$$

and its complementary part, the point spectrum

$$
\operatorname{spec}_{\mathrm{pt}} \mathcal{L}_{*}:=\operatorname{spec} \mathcal{L}_{*} \backslash \operatorname{spec}_{\text {ess }} \mathcal{L}_{*} .
$$

The appearance of essential spectrum is caused by the non-compactness of the real line $x \in \mathbb{R}$, here. By robustness of Fredholm properties, the essential spectrum is closed. As usual, for $\lambda \in \operatorname{spec}_{\text {pt }} \mathcal{L}_{*}$, nontrivial $u \in \operatorname{Ker}\left(\mathcal{L}_{*}-\lambda\right)$ are then called eigenfunctions and algebraic multiplicity is associated to Jordan blocks. Due to analyticity of the eigenvalue problem in $\lambda$, the only possible degeneracy in this description of point spectrum is characterized by connected components of the set of $\lambda$ such that the Fredholm index of $\mathcal{L}_{*}-\lambda$ is zero and the kernel is nontrivial; see [Ka66, He81].

For example, consider the heteroclinic case of a travelling front $q_{*}$, where $q_{*}(\xi) \rightarrow q_{ \pm}$for $\xi \rightarrow \pm \infty$. In Proposition 2.3.1, below, we will characterize the essential spectrum of $\mathcal{L}_{*}$ on $L^{2}(\mathbb{R})$ in terms of the spectra of the asymptotic linearizations $\mathcal{L}_{ \pm}$at $q_{ \pm}$:

$$
\mathcal{L}_{ \pm} w:=D w_{\xi \xi}+c w_{\xi}+f^{\prime}\left(q_{ \pm}\right) w .
$$

Unlike $\mathcal{L}_{*}$, the differential operators $\mathcal{L}_{ \pm}$possess constant, $x$-independent coefficients. The spectra of $\mathcal{L}_{ \pm}$are therefore readily computed in terms of exponentials. Setting $w(\xi)=\mathrm{e}^{-\nu \xi} w_{0}$, with $\nu \in \mathbb{C}$, the spectral problem $\mathcal{L}_{ \pm} w=\lambda w$ is transformed into the complex dispersion relation 


$$
0=d_{ \pm}(\lambda, \nu):=\operatorname{det}\left(D \nu^{2}-c \nu+f^{\prime}\left(q_{ \pm}\right)-\lambda\right)
$$

which is polynomial in the complex variables $\lambda, \nu \in \mathbb{C}$. For fixed spatial eigenvalue $\nu$, we find $N$ roots $\lambda_{\ell}=\lambda_{\ell}(\nu)$. Conversely, fixing the temporal eigenvalue $\lambda$, we find $2 N$ roots $\nu_{j}=\nu_{j}(\lambda)$. By Rouché's theorem, the roots depend continuously on $\nu$ or $\lambda$, respectively. We recover the Fourier transform in $x$ when specializing to $\nu=\mathrm{i} k$ with real spatial wavenumber $k \in \mathbb{R}$. We therefore conclude that

$$
\lambda \in \operatorname{spec} \mathcal{L}_{ \pm} \quad \Longleftrightarrow \quad d_{ \pm}(\lambda, \mathrm{i} k)=0 \text { for some } k \in \mathbb{R}
$$

The spectra of $\mathcal{L}_{ \pm}$then each decompose into $N$ algebraic curves

$$
\operatorname{spec} \mathcal{L}_{ \pm}=\bigcup_{\ell} \Gamma_{ \pm}^{\ell}, \quad \Gamma_{ \pm}^{\ell}=\left\{\lambda_{ \pm}^{\ell}(\mathrm{i} k) ; k \in \mathbb{R}\right\}
$$

The imaginary part of the tangent vector to any dispersion curve $\lambda_{ \pm}^{\ell}(\mathrm{i} k)$, $k \in \mathbb{R}$, is called its group velocity $c_{\mathrm{g}}$ :

$$
c_{\mathrm{g}, \pm}^{\ell}(k)=\operatorname{Im} \frac{\mathrm{d} \lambda_{ \pm}^{\ell}(\mathrm{i} k)}{\mathrm{d} k} .
$$

From the dispersion relation (2.67), the sum $c_{\mathrm{g}, \pm}^{\ell}(k)+c$ is independent of the speed $c$ of the coordinate frame.

For an interpretation, first consider the special case of purely imaginary temporal eigenvalues $\lambda_{ \pm}^{\ell}(\mathrm{i} k)$. For classical linear conservative wave equations like $u_{t}=u_{x}, u_{t t}=u_{x x}$, the Schrödinger equation, etc., the dispersion curve $\Gamma$ is indeed vertical. Then the group velocity $c_{\mathrm{g}}$ describes the speed and direction of $x$-propagation of an initial condition, which consists of a narrow Gaussian wave package of spatial oscillations $\mathrm{e}^{\mathrm{i} k x}$. The same observation holds true in the dissipative context, for $\lambda_{ \pm}^{\ell}(\mathrm{i} k)$ with nonvanishing real part. The real part $\operatorname{Re} \lambda_{ \pm}^{\ell}(\mathrm{i} k)$ then just produces a superimposed exponential temporal growth or decay of the wave packet, which moves along the $x$-axis with its group velocity $c_{\mathrm{g}}$.

We orient the curves $\Gamma_{ \pm}^{\ell}$ in the direction of increasing $k$. Upwards orientation of $\Gamma_{ \pm}^{\ell}$, in the complex plane, therefore indicates a positive group velocity, and downwards orientation a negative group velocity. The following proposition identifies the curves $\Gamma_{ \pm}^{\ell}$ as Fredholm borders, where the Fredholm index of $\mathcal{L}_{*}$ changes.

Proposition 2.3.1 The operator $\mathcal{L}_{*}-\lambda$ on $L^{2}(\mathbb{R})$ is Fredholm for $\lambda$ in the complement of $\operatorname{spec} \mathcal{L}_{+} \cup \operatorname{spec} \mathcal{L}_{-}$. The Fredholm index increases by one upon crossing a curve $\lambda_{+}^{\ell}(\mathrm{i} k)$ from left to right, with respect to its given orientation; it decreases by one upon crossing a curve $\lambda_{-}^{\ell}(\mathrm{i} k)$ from left to right.

For example, assume that the group velocity $c_{\mathrm{g},+}^{\ell}(k)$ or $c_{\mathrm{g},-}^{\ell}(k)$ is directed towards the front position, which is stationary in our comoving coordinates: 
$\pm c_{\mathrm{g}, \pm}^{\ell}(k)<0$. Then the Fredholm index of $\mathcal{L}_{*}-\lambda$ decreases when $\operatorname{Re} \lambda$ increases across the spectral curve $\lambda_{+}^{\ell}(\mathrm{i} k)$ or $\lambda_{-}^{\ell}(\mathrm{i} k)$, respectively. Conversely, if the group velocity is directed away from the interface, $\pm c_{\mathrm{g}, \pm}^{\ell}(k)>0$, then the Fredholm index of $\mathcal{L}_{*}-\lambda$ increases when $\operatorname{Re} \lambda$ increases across the spectral curve $\lambda_{+}^{\ell}(\mathrm{i} k)$ or $\lambda_{-}^{\ell}(\mathrm{i} k)$, respectively; see Figure 2.4. In particular, the Fredholm index is +1 to the left of the most unstable curve $\Gamma_{ \pm}^{\ell}$, with $\operatorname{Re} \lambda$ maximal for all choices of $\ell, \pm$, if the associated group velocity is directed towards the front. The Fredholm index is -1 if it is directed away from the front.

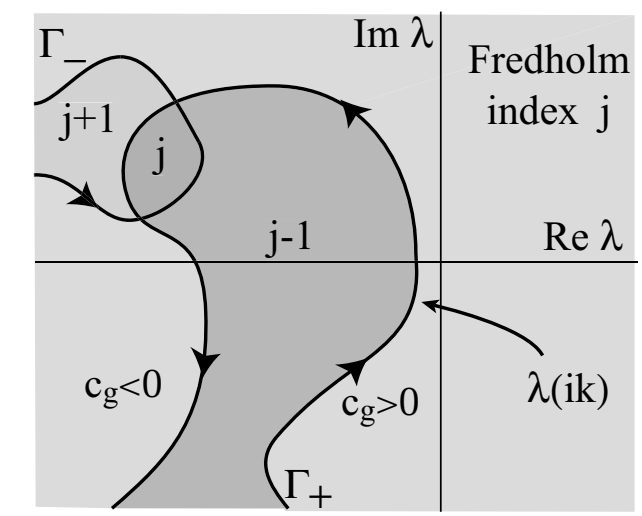

Fig. 2.4. A schematic plot of the oriented spectral curves $\Gamma_{ \pm}^{\ell}$ and the Fredholm indices of $\mathcal{L}_{*}-\lambda$.

The proof of Propostion 2.3.1 is based on a formulation of the spectral problem $\left(\mathcal{L}_{*}-\lambda\right) u$ as a first-order differential equation

$$
u_{\xi}=v, \quad v_{\xi}=D^{-1}\left(-c v-f^{\prime}\left(q_{*}(\xi)\right) u+\lambda u\right) .
$$

Consider therefore a generalized eigenvalue problem for first-order differential operators

$$
\mathcal{M}(\lambda) \mathbf{w}:=\frac{\mathrm{d} \mathbf{w}}{\mathrm{d} \xi}-\mathbf{A}(\xi ; \lambda) \mathbf{w}=0 .
$$

Assume that the matrices $\mathbf{A}(x ; \lambda)$ are asymptotically constant: $\mathbf{A}(x ; \lambda) \rightarrow$ $\mathbf{A}_{ \pm}(\lambda)$ for $x \rightarrow \pm \infty$. Note that the asymptotic eigenvalues $\nu_{ \pm}^{\ell}(\lambda)$ of $\mathcal{A}_{ \pm}(\lambda)$ satisfy the dispersion relation

$$
d_{ \pm}\left(\lambda, \nu_{ \pm}^{\ell}(\lambda)\right):=\operatorname{det}\left(-\nu_{ \pm}^{\ell}-\mathbf{A}_{ \pm}(\lambda)\right)=0 .
$$

We define the asymptotic, spatial Morse indices $i_{ \pm}(\lambda)$ as the dimensions of the unstable eigenspaces of $\mathbf{A}_{ \pm}(\lambda)$, respectively. Note that from the Ansatz $\mathbf{w}(\xi)=\mathrm{e}^{-\nu \xi} \mathbf{w}_{0}$, the spatial Morse index is given by the number of roots $\nu$ of the dispersion relation for given $\lambda$, with $\operatorname{Re} \nu<0$. 
Proposition 2.3.2 The operator $\mathcal{M}(\lambda)=\frac{\mathrm{d}}{\mathrm{d} \xi}-\mathbf{A}(\xi ; \lambda)$, considered as a closed, unbounded operator on $L^{2}(\mathbb{R})$, is Fredholm if, and only if, $\operatorname{Re} \nu_{ \pm}^{j} \neq 0$ for all $j$. The Fredholm index is given by

$$
i(\mathcal{M}(\lambda))=i_{-}(\lambda)-i_{+}(\lambda),
$$

where the spatial Morse indices $i_{ \pm}(\lambda)=\#\left\{\operatorname{Re} \nu_{ \pm}^{j}<0\right\}$ count the number of unstable eigenvalues of the asymptotic matrices $\mathbf{A}_{ \pm}(\lambda)$, with algebraic multiplicity.

In the formulation as a first order, generalized eigenvalue problem, the proposition actually covers a much broader class of linear operators than linearizations about travelling waves in reaction-diffusion equations. We refer to [SaSc01d] for a still more general formulation, which allows to cover linearizations about time-periodic states as well as problems in several space dimensions $m>1$.

Essential spectra do not depend on function spaces such as $L^{p}, C^{0}$, or $H^{k}$, as long as translation invariant norms are considered. However, the locations of the Fredholm borders $\Gamma_{ \pm}^{\ell}$ do change when we consider spaces with exponential weights. For weights $\underline{\eta}=\left(\eta_{+}, \eta_{-}\right) \in \mathbb{R}^{2}$, define

$L_{\underline{\eta}}^{2}:=\left\{u \in L_{\mathrm{loc}}^{2} ;\|u\|_{L_{\eta}^{2}}<\infty\right\}, \quad\|u\|_{L_{\underline{\eta}}^{2}}^{2}:=\int_{\mathbb{R}^{+}}\left|\mathrm{e}^{\eta_{+} \xi} u(\xi)\right|^{2}+\int_{\mathbb{R}^{-}}\left|\mathrm{e}^{\eta_{-} \xi} u(\xi)\right|^{2}$.

Positive weights $\eta_{ \pm}>0$ stabilize transport to the left. For example, consider pure transport to the left by $u_{t}=u_{\xi}$, on the entire real line. The trivial solution $q_{*} \equiv 0$ is then stabilized by an exponential weight with $\eta:=\eta_{+}=\eta_{-}>0$. Indeed, the exponentially weighted solution $v(t, \xi)=\mathrm{e}^{\eta \xi} u(t, \xi)$ satisfies $\|u\|_{L_{\underline{\eta}}^{2}}=\|v\|_{L^{2}}$, for all fixed $t$. Moreover, $v$ solves the damped transport equation $v_{t}=v_{\xi}-\eta v$, and hence decays to zero exponentially, in $L^{2}(\mathbb{R})$, with rate $\eta>0$.

More generally, transport is measured by the group velocity, as reflected by the following lemma.

Lemma 2.3.3 The essential spectrum in $L_{\eta}^{2}$ can be determined from Proposition 2.3.1, if we replace the dispersion relations (2.67) at the asymptotic states by the shifted dispersion relations

$$
d_{ \pm}^{\eta}(\lambda, \nu):=d_{ \pm}\left(\lambda, \nu-\eta_{ \pm}\right) .
$$

In particular, small weights $\eta_{ \pm}$infinitesimally shift the Fredholm borders $\Gamma_{ \pm}^{\ell}=\left\{\lambda_{ \pm}^{\ell}\left(\mathrm{i} k ; \eta_{ \pm}\right) ; k \in \mathbb{R}\right\}$ as follows:

$$
\left.\frac{\partial \operatorname{Re} \lambda_{ \pm}^{\ell}(\mathrm{i} k ; \eta)}{\partial \eta}\right|_{\eta_{ \pm}=0}=-c_{\mathrm{g}, \pm}^{\ell}(k)
$$


The key observation in the proof of Lemma 2.3 .3 is, that $\lambda_{ \pm}^{\ell}(\mathrm{i} k ; \eta)=\lambda_{ \pm}^{\ell}(\mathrm{i} k-$ $\eta ; 0)$, not only for real but also for complex $k$. Therefore

$$
\begin{aligned}
& \left.\frac{\partial \operatorname{Re} \lambda_{ \pm}^{\ell}(\mathrm{i} k ; \eta)}{\partial \eta}\right|_{\eta=0}= \\
& \left.\frac{\partial \operatorname{Re} \lambda_{ \pm}^{\ell}(\mathrm{i} k-\eta ; 0)}{\partial \operatorname{Im} k}\right|_{\eta=0}=-\left.\frac{\partial \operatorname{Im} \lambda_{ \pm}^{\ell}(\mathrm{i} k-\eta ; 0)}{\partial \operatorname{Re} k}\right|_{\eta=0}=-c_{\mathrm{g}, \pm}^{\ell}(k) .
\end{aligned}
$$

Here, we have used the Cauchy-Riemann equations for complex analytic functions in the second identity.

Proofs of Propositions 2.3.1 and 2.3.2 can be found, in a slightly different context, in [Pa88, He81]; see also [SaSc01d] for a more general setup. We conclude this section on essential spectra with several examples.

Consider the linear scalar reaction-advection-diffusion equation

$$
w_{t}=w_{\xi \xi}+w_{\xi}+a w=: \mathcal{L}_{*} w
$$

with positive drift velocity and constant reaction coefficient $a \in \mathbb{R}$. We first view $\mathcal{L}_{*}$ as an unbounded operator on $L^{2}(\mathbb{R})$, as in Propositions 2.3.1 and 2.3.2.

From (2.67) and (2.68), we obtain the dispersion relation and spectrum of $\mathcal{L}_{*}$ to be given by the parabola

$$
0=d(\lambda, \mathrm{i} k)=-k^{2}-\mathrm{i} k+a-\lambda ;
$$

$\operatorname{spec} \mathcal{L}_{*}=\left\{\lambda=-k^{2}+a+\mathrm{i} k ; k \in \mathbb{R}\right\}=\left\{\lambda \in \mathbb{C} ; \operatorname{Re} \lambda=(\operatorname{Im} \lambda)^{2}+a\right\}=: \Gamma$.

Preparing for the more general case, where $a$ depends on $\xi$, we now consider (2.75) on $\xi \in \mathbb{R}_{+}$or $\mathbb{R}_{-}$and equip the arising differential operators $\mathcal{L}_{>}$and $\mathcal{L}_{<}$with Dirichlet boundary conditions in $\xi=0$. We claim that

$$
\operatorname{spec} \mathcal{L}_{>}=\operatorname{spec} \mathcal{L}_{<}=\left\{\lambda \in \mathbb{C} ; \operatorname{Re} \lambda \leq(\operatorname{Im} \lambda)^{2}+a\right\},
$$

with Fredholm index $i\left(\mathcal{L}_{>}-\lambda\right)=1$ and $i\left(\mathcal{L}_{<}-\lambda\right)=-1$ in the interior of the spectrum. Indeed, we follow $(2.70)$ to rewrite $\left(\mathcal{L}_{>}-\lambda\right) w=0$ as a first order system

$$
\mathcal{M}(\lambda) \mathbf{w}=\mathbf{w}_{\xi}+\mathbf{A}(\lambda) \mathbf{w}=0, \quad \mathbf{A}(\lambda)=\left(\begin{array}{cc}
0 & -1 \\
a-\lambda & 1
\end{array}\right),
$$

for $\xi>0$. For $\operatorname{Re} \lambda<(\operatorname{Im} \lambda)^{2}+a$, to the left of the Fredholm border $\Gamma$, both eigenvalues $\nu^{1,2}(\lambda)$ of $\mathbf{A}(\lambda)$ possess strictly positive real part. The onedimensional subspace in the $\mathbf{w}$-plane, selected by the Dirichlet boundary conditions at $\xi=0$ therefore gives rise to a one-dimensional kernel of exponentially decaying functions. The operator $\mathcal{L}_{>}$is surjective since solutions to the initial value problem 


$$
\mathbf{w}_{\xi}=-\mathbf{A}(\lambda) \mathbf{w}+\mathbf{h}(\xi)
$$

with $\mathbf{w}(0)=0$ for $\xi>0$ belong to $L^{2}$. This shows that $\mathcal{L}_{>}-\lambda$ is Fredholm of index 1 to the left of the Fredholm border. The operator $\mathcal{L}_{<}$is conjugate to the adjoint of $\mathcal{L}_{>}$on $\mathbb{R}^{+}$, by the reflection $x \mapsto-x$, and therefore possesses Fredholm index -1. For $\lambda$ to the right of the Fredholm border, both operators are invertible since the Dirichlet subspace does not coincide with an eigenspace of $\mathbf{A}(\lambda)$.

Next consider piecewise constant coefficients $a(\xi)=a_{ \pm}$for $\pm \xi>0$ in the advection-diffusion equation (2.75). We then obtain corresponding spectral borders $\Gamma_{ \pm} \subset \mathbb{C}$, which are parabolas shifted by $a_{ \pm}$, respectively. Assume $a_{+}>a_{-}$, first. We claim that

$$
\operatorname{spec} \mathcal{L}_{*}=\left\{\lambda \in \mathbb{C} ; \quad(\operatorname{Im} \lambda)^{2}+a_{-} \leq \operatorname{Re} \lambda \leq(\operatorname{Im} \lambda)^{2}+a_{+}\right\},
$$

with Fredholm index $i\left(\mathcal{L}_{*}-\lambda\right)=1$ in the interior of the spectrum. Indeed, consider the first-order eigenvalue problem (2.78) with $\lambda$ between the spectral borders $\Gamma_{ \pm}$. The eigenvalues of the asymptotic matrices $\mathbf{A}_{ \pm}$then satisfy $\operatorname{Re} \nu_{-}^{1}<0<\operatorname{Re} \nu_{-}^{2}$ and $\operatorname{Re} \nu_{+}^{1,2}>0$. The differential equation therefore possesses a one-dimensional subspace of solutions $\mathbf{w}(\xi)$ which are continuous in $\xi=0$ and decay for $\xi \rightarrow \pm \infty$. This contributes a one-dimensional kernel in the Fredholm index one region of $\mathcal{L}_{*}$. Surjectivity between the Fredholm borders, and invertibility in the Fredholm index zero region, follow just like for the operator $\mathcal{L}_{>}$.

In the opposite case $a_{-}>a_{+}$, the Fredholm index is -1 between the Fredholm borders $\Gamma_{ \pm}$. In the interpretation of Proposition 2.3.1, the group velocity $c_{\mathrm{g}}=-1$ is constant and negative on $\Gamma_{+}$, directed towards the "front" in $\xi=0$. The Fredholm index therefore decreases from left to right. However, on $\Gamma_{-}$the group velocity $c_{\mathrm{g}}=-1$ is directed away from the "front", and the Fredholm index increases from left to right.

Essential spectra for more general coefficients $a=a(\xi)$ with $a(\xi) \rightarrow$ $a_{ \pm}$coincide with the essential spectra computed above, since localized $\xi$ dependence of $a(\xi)$ amounts to a relatively compact perturbation of $\mathcal{L}_{*}$, which does not change Fredholm properties [Ka66].

Just like in the example of simple translation $u_{t}=u_{\xi}$, exponential weights $\underline{\eta}=\left(\eta_{+}, \eta_{-}\right)$with $\eta_{+}, \eta_{-}>0$ shift the Fredholm borders to the left, as can be readily seen from the complex dispersion relations, or Lemma 2.3.3.

As a final example, we comment on spectra in the case of spatially periodic coefficients $a=a(\xi)=a(\xi+p)$. This situation arises under linearization $a(\xi)=f^{\prime}\left(q_{\mathrm{wt}}(\xi)\right)$ along a periodic wavetrain with nonlinear spatial wave vector $k_{\mathrm{wt}}=2 \pi / p$, in comoving coordinates $\xi$; see for example the end of Section 2.3.2. In a more demanding context, the problem reappears in our stability analysis of Archimedean spiral waves, for radial spatial dynamics in the farfield limit of infinite radius; see Section 2.4.2, 2.5.3, and equations (2.123)-(2.125). Standard spatial Floquet theory for the eigenvalue problem 


$$
u_{\xi}=v, \quad v_{\xi}=-c v-a(\xi) u-\lambda u
$$

will consider exponential functions $\mathbf{w}=(u, v)$,

$$
\mathbf{w}(\xi)=\mathrm{e}^{-\nu \xi} \tilde{\mathbf{w}}(\xi)
$$

with spatially $p$-periodic $\tilde{\mathbf{w}}$ and spatial Floquet exponent $\nu \in \mathbb{C}$. As usual, $\nu$ is only determined up to integer multiples of $2 \pi \mathrm{i} / p=\mathrm{i} k_{\mathrm{wt}}$. Explicitly,

$$
\left(\partial_{\xi}-\nu\right)^{2} \tilde{\mathbf{w}}+\left(\partial_{\xi}-\nu\right) \tilde{\mathbf{w}}+a(\xi) \tilde{\mathbf{w}}=\lambda \tilde{\mathbf{w}}
$$

Alternatively, we can work with w directly and impose the Floquet boundary condition

$$
\mathbf{w}(p)=\mathrm{e}^{-\nu p} \mathbf{w}(0) \text {. }
$$

The Floquet exponents $\nu$ play the role of the spatial wave numbers in the dispersion relation (2.67). Looking for solutions with neutral growth $\nu=\mathrm{i} k$, $k \in \mathbb{R}$, in analogy to the spectral results of Propositions 2.3.1, 2.3.2, we obtain the dispersion relation

$$
d(\lambda, \mathrm{i} k)=\operatorname{det}\left(\Phi(\lambda)-\mathrm{e}^{-\mathrm{i} k p}\right)=0,
$$

where $\Phi(\lambda)$ denotes the period map to the linear differential equation (2.80). Note that the dispersion relation is invariant under the Floquet shift $k \rightarrow$ $k+k_{\mathrm{wt}}$. The values of $k$ in the dispersion relation (2.83) are referred to as Bloch wavenumbers. The associated spectral values $\lambda^{\ell}(\mathrm{i} k), \ell=1, \ldots, N$, are the eigenvalues of the elliptic operator $\mathcal{L}_{*}$ on $L^{2}(\mathbb{R})$. The eigenfunctions $u(\xi)$ are called Bloch waves associated with the Bloch wavenumber $k$. We can also define group velocities

$$
c_{\mathrm{g}}^{\ell}(k):=\frac{\mathrm{d} \operatorname{Im} \lambda^{\ell}(\mathrm{i} k)}{\mathrm{d} k},
$$

in analogy to (2.69). Analogous statements to Propositions 2.3.1, 2.3.2 then hold for equations with asymptotically periodic coefficients, with Fredholm borders $\Gamma^{\ell}$ defined through $(2.83)$.

\subsubsection{Instabilities of Travelling Waves}

In this section, we investigate relative equilibria which lose stability for the PDE (2.64)

$$
u_{t}=D u_{x x}+f(u ; \mu)
$$

on the unbounded real line $x \in \mathbb{R}$. Here, $\mu \in \mathbb{R}$ denotes a typical control parameter, driving the instability, which we assume to occur at the value $\mu=0$. Instabilities can be caused either by point spectrum or by essential spectrum crossing the imaginary axis. Without striving for completeness, we present four different cases of Hopf bifurcation from travelling waves, here. Three of them are caused by essential spectrum crossing the imaginary axis. 
Fredholm indices play a most prominent role when solving the resulting nonlinear equations, locally. Lyapunov-Schmidt reduction generally provides reduced equations, which map the kernel to the cokernel. Because the boundaries of the essential spectrum were characterized precisely by the loss of Fredholm property, in Section 2.3.1, we will rely on spatial dynamics ideas, in the spirit of [Ki82, Fi84] to discuss these latter bifurcations.

Instability of a front caused by point spectrum First consider a travelling wave $u=q_{*}\left(x-c_{*} t\right)$ with essential spectrum of the linearization $\mathcal{L}_{*}$ strictly contained in the open left half-plane, see (2.64),(2.68). We assume that there is a pair of simple, purely imaginary eigenvalues crossing the imaginary axis. In addition, there necessarily is a zero eigenvalue due to translation of the relative equilibrium $q_{*}$. This additional eigenvalue prevents the straight-forward application of standard Hopf bifurcation theorems. We choose to proceed by center manifold reduction, instead. With a careful choice of cut-off functions, we can construct a center manifold which is invariant under translations [He81, Sa\&al97b]. With an appropriate choice of coordinates, we find a three-dimensional reduced system of ODEs on the center manifold, which is of skew-product form

$$
\dot{v}=h(v ; \mu), \quad \dot{g}=c(v ; \mu) .
$$

Here, $v \in \mathcal{U} \subset \mathbb{C}, g \in \mathbb{R}, h(0 ; 0)=0$ and $\operatorname{spec} h_{v}(0 ; 0)=\{ \pm \mathrm{i} \omega\}$. The (small) $v$-variable parameterizes the Hopf eigenspace. The (global) variable $g$ in the translation group $G=\mathbb{R}$ parameterizes the position of the front. Since we can preserve the translation equivariance of the original reaction-diffusion system (2.64) through the center manifold reduction, the Hopf bifurcation in the $v$ equation is independent of the translation component $g$. As a result, we typically encounter a generic Hopf bifurcation in the $v$-equation. Unique periodic orbits $v_{*}(t ; \mu)$ arise super- or subcritically, depending only on the sign of a certain cubic normal form coefficient. The $v$-dynamics enter the $g$-equation, with the periodic solution $v_{*}(t ; \mu)$ acting as a time-periodic forcing. Phenomenologically, this leads to a periodically oscillating speed $c=c\left(v_{*}(t ; \mu) ; \mu\right)$ of the wave front position. The periodic variable $v_{*}(t ; \mu)$ indicates small periodic shape fluctuations of the propagating wave.

We refer to Section 2.5.2 for a thorough discussion in the more complicated, but analogous situation of meandering spiral wave patterns.

The Turing instability When the essential spectrum of the linearization $\mathcal{L}_{*}$ at a front touches the imaginary axis, a naive finite-dimensional LyapunovSchmidt reduction fails, due to the absence of the Fredholm property. The dynamics, generated by a continuum of eigenmodes, cannot be represented by a finite collection of ordinary differential equations. We illustrate the effects of critical essential spectrum as opposed to critical point spectrum by means of an example: one of the asymptotic states of a propagating front experiences a Turing instability. We begin with some background on the nature 
of this pattern forming mechanism, predicted by Turing in 1952 [Tu52], and observed, only in 1989, in laboratory experiments [Ca\&al90].

A Turing instability occurs at one of the asymptotic states $q_{ \pm}$if the spectrum of $\mathcal{L}_{ \pm}$in the steady frame touches the imaginary axis at $\lambda=0$, with spatially inhomogeneous critical eigenfunction. To fix ideas, we assume that the Turing instability occurs at $q_{+}$and we set $q_{+}(\mu) \equiv 0$. We consider the reaction-diffusion system (2.64) in the steady frame, first

$$
u_{t}=D u_{x x}+f(u ; \mu), \quad x \in \mathbb{R},
$$

with linearization

$$
\mathcal{L}_{0}(\mu)=D u_{x x}+f^{\prime}(0 ; \mu) u \text {. }
$$

In the notation of Section 2.3.1, one of the curves $\Gamma_{+}^{\ell}$ touches the imaginary axes with a zero eigenvalue $\lambda\left(\mathrm{i} k_{*}\right)=0$, for nonzero wavenumber, $k_{*} \neq 0$; see Figure 2.5. We assume that $\lambda^{\ell}\left(k_{*} ; \mu=0\right)=0$ is a simple root of the
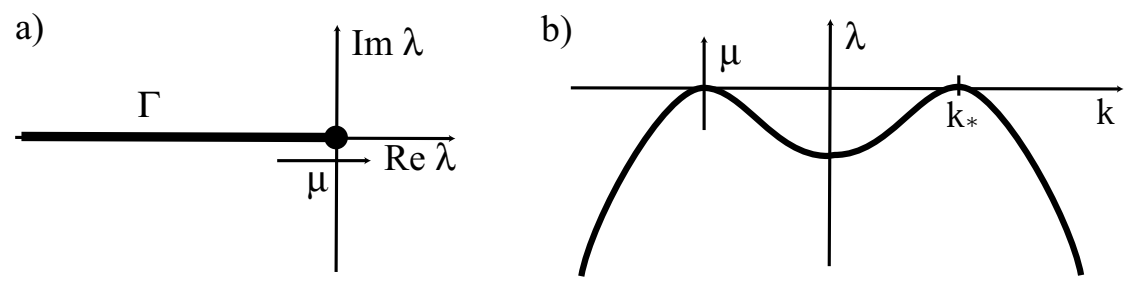

Fig. 2.5. The critical curve $\Gamma_{+}^{\ell} \subset \mathbb{C},(\mathbf{a})$, and the temporal eigenvalue $\lambda_{+}^{\ell}$ as a function of the wavenumber $k,(\mathbf{b})$, are shown at criticality.

dispersion relation (2.67), which crosses the imaginary axis with nonvanishing speed. After a suitable scaling, we find

$$
\lambda^{\ell}\left(k ; \mu_{0}\right)=\mu-\left(k-k_{*}\right)^{2}+\mathrm{O}\left(\left(k-k_{*}\right)^{3}\right),
$$

for $k \sim k_{*}$. Since $\mathcal{L}_{0}$ possesses real coefficients, the curve $\Gamma_{+}^{\ell}$ is necessarily contained in the real axis close to the origin.

It was Turing's original discovery that this instability can actually occur in reaction-diffusion systems, although the spatially homogenizing effect of diffusion suggests that the most unstable eigenfunction ought to be spatially homogeneous. In case of a Turing instability, a spatially structured eigenfunction $u_{*} \cos \left(k_{*} x\right)$ is amplified by the time evolution for the linearization of the reaction-diffusion system (2.64) about the critical state $q_{+}=0$

$$
u_{t}=D u_{x x}+f^{\prime}(0 ; 0) u .
$$

The resulting spatially periodic, stationary pattern for the nonlinear equation (2.64) near 0 is generally referred to as a Turing pattern $T(x)$. 
One possible theoretical approach to this instability imposes spatial periodicity with period $L \sim 2 \pi / k_{*}$ in the function space, restricting the allowed values of the wavenumber of $k$ to multiples of $2 \pi / L$; see [Go\&al88]. Then the Turing bifurcation reduces to a pitchfork bifurcation in the space of even functions with prescribed period $L$, with reduced equation on a center manifold given by

$$
\dot{z}=\left(\mu-\left(k-k_{*}\right)^{2}\right) z+\beta(k ; \mu) z^{3}+\mathrm{O}\left(z^{5}\right) \in \mathbb{R} .
$$

We will assume throughout that $\beta\left(k_{*} ; 0\right)<0$, such that the bifurcating solutions are asymptotically stable - in the space of functions with the prescribed period $2 \pi / k$.

In a slightly different spirit, Turing instabilities on unbounded domains, without imposing periodicity, have been described by a partial differential equation instead of our single ordinary differential equation. The dynamics of the reaction-diffusion system can be approximated by a slow, long-wavelength modulation of the complex amplitude of the critical eigenfunction:

$$
u(t, x) \sim \sqrt{\mu}\left(A(\mu t, \sqrt{\mu} x) \mathrm{e}^{\mathrm{i} k_{*} x}+\bar{A}(\mu t, \sqrt{\mu} x) \mathrm{e}^{-\mathrm{i} k_{*} x}\right) u_{*} .
$$

The time evolution of the complex amplitude $A(\tilde{t}, \tilde{x}) \in \mathbb{C}$ is to leading order given by a Ginzburg-Landau equation

$$
A_{\tilde{t}}=A_{\tilde{x} \tilde{x}}+A-A|A|^{2}
$$

on time scales $\tilde{t}=\mathrm{O}(1)$. In the Ginzburg-Landau description (2.88), the stationary Turing patterns are solutions of the form $A(\tilde{x})=r(\tilde{k}) \mathrm{e}^{\mathrm{i} \tilde{k} \tilde{x}}$. Their linearized stability can be explicitly calculated. In $(\mu, \tilde{k})$-parameter space, only Turing patterns which are sufficiently close to the critical wavenumber, $k=k_{*}$, or $\tilde{k}=0$, are stable — with respect to not necessarily spatially periodic perturbations. The boundary of stability is commonly referred to as the Eckhaus boundary; see Figure 2.6. Turing patterns $T(x ; \mu, k)$ exist

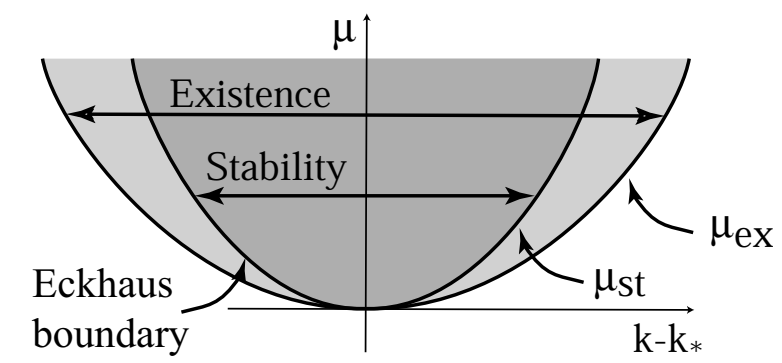

Fig. 2.6. Existence and stability of Turing patterns depending on spatial wavenumber $k$ and bifurcation parameter $k$.

for parameter values $\mu>\mu_{\mathrm{ex}}\left(k-k_{*}\right)$, and are asymptotically stable for 
$\mu>\mu_{\mathrm{st}}\left(k-k_{*}\right) \geq \mu_{\mathrm{ex}}\left(k-k_{*}\right)$. The boundary curves can be computed to leading order from the Ginzburg-Landau approximation (2.88):

$$
\mu_{\mathrm{ex}}(\tilde{k})=\tilde{k}^{2}+\mathrm{O}\left(\tilde{k}^{3}\right), \quad \mu_{\mathrm{st}}(\tilde{k})=3 \tilde{k}^{2}+\mathrm{O}\left(\tilde{k}^{3}\right) .
$$

We refer to [CrHo93, MiSc96, Mi97, SaSc00b, Sc98c] for physics background and mathematical proofs in this context.

Essential Hopf instability of a front We are now going to describe a general bifurcation result for a front, where one of the asymptotic states undergoes a Turing instability. Reflecting the spatial variable $x$, if necessary, we may fix $c_{*}>0$, that is, propagation towards $x=+\infty$. Then the direction of propagation of the front distinguishes between the asymptotic states $q_{ \pm}$. We refer to $q_{+}$as the state ahead of the front, and to $q_{-}$as the state which is behind the front.

Whereas the Turing bifurcation is stationary in the steady frame, the solution $u_{*} \operatorname{expi} k_{*} x$ to the linearized equation (2.86) becomes time-periodic $u_{*} \exp \left(\mathrm{i} k_{*} \xi+\mathrm{i} \omega_{*} t\right)$, in a comoving frame with speed $c_{*}=\omega_{*} / k_{*}>0$. Viewed in the comoving frame, the Turing bifurcation therefore becomes a Hopf bifurcation. Typically, in a Hopf bifurcation, we are interested in time-periodic solutions. More specifically, we shall be interested in the existence and stability of modulated front solutions to the reaction-diffusion system (2.64):

$$
u(t, x)=q(t, x-c t), \quad \text { with } q(t, \xi)=q(t+P, \xi), \text { for all } t, \xi \in \mathbb{R},
$$

for some temporal period $P$, and an appropriate wave speed $c \sim c_{*}$.

Setting up a typical bifurcation result usually requires existence of a primary solution branch, minimal critical spectrum, and assumptions on nonlinear terms in the Taylor expansion. We are now going to make these assumptions precise for our set-up. Recall that $x$ always refers to the spatial coordinate in the steady frame, whereas $\xi$ always refers to some comoving frame.

At criticality $\mu=0$, we assume existence of a uniformly translating front solution, $q\left(x-c_{*} t\right) \rightarrow q_{ \pm}$for $x \rightarrow \pm \infty$ for some strictly positive speed of propagation $c_{*}>0$.

The critical spectrum is assumed to generate a Turing instability at one of the asymptotic states, either ahead of the front, at $q_{+}$, or behind the front, at $q_{-}$. In the comoving frame $\xi=x-c_{*} t$, this Turing instability corresponds to a spectral curve $\Gamma_{+}$(or $\Gamma_{-}$, respectively) of the linearization about the front $\mathcal{L}_{*},(2.66)$ which touches the imaginary axis. The tangency at $\lambda\left(\mathrm{i} k_{*}\right)=\mathrm{i} \omega_{*} \neq 0$ is quadratic from the left. From the dispersion relation (2.67), we find $\omega_{*}=c_{*} k_{*}$. Increasing the parameter $\mu$ through zero, we assume that $\Gamma_{+}$(or $\Gamma_{-}$, respectively) crosses the imaginary axis with nonzero speed.

Minimal spectrum at bifurcation, here, refers to the spectrum of the front in a comoving frame: we assume that the critical spectrum only consists of the part related to the Turing instability and the simple eigenvalue $\lambda=0$, 
induced by translation. More precisely, we assume that $\lambda\left(\mathrm{i} k_{*}\right)= \pm \mathrm{i} \omega_{*}$ are the only roots of the dispersion relation (2.67) in the comoving frame $c=c_{*}$ on the imaginary axis $\lambda \in \mathrm{i} \mathbb{R}$, and $\lambda= \pm \mathrm{i} \omega_{*}$ are simple roots. Moreover, we require absence of critical point spectrum on the imaginary axis: in an exponentially weighted space $L_{\eta}^{2}(\mathbb{R})$ with norm

$$
\|u\|_{L_{\eta}^{2}}^{2}:=\int_{\mathbb{R}}\left|\mathrm{e}^{\eta \xi} u(\xi)\right|^{2}<\infty
$$

we assume that

$$
\operatorname{spec}_{L_{\eta}^{2}} \mathcal{L}_{*} \cap \mathrm{i} \mathbb{R}=\{0\},
$$

is algebraically simple, for all $\eta>0$ sufficiently small. Note that compared to Lemma 2.3.3, we used exponential weights $\eta_{+}=\eta_{-}:=\eta$.

Nonlinear terms for the Turing bifurcation in a neighborhood of the asymptotic state $q_{+}$(or $q_{-}$, respectively) are most easily computed in the steady frame, restricting to even, $2 \pi / k$-periodic functions. As mentioned above, the instability then induces a pitchfork bifurcation on a onedimensional center manifold, with Taylor expansion

$$
\dot{z}=\lambda(\mathrm{i} k ; \mu) z+\beta(k ; \mu) z^{3}+\mathrm{O}\left(z^{5}\right) .
$$

We assume $\beta\left(k_{*} ; 0\right)<0$ such that the bifurcating Turing patterns are stable within this class of periodic functions. From elementary bifurcation theory, we find for each $k \sim k_{*}$ and $\mu>0, \mu \sim 0$, a periodic Turing pattern $T(x ; \mu, k)$, which is time-independent in the original frame $x$ and unique up to translation in $x$.

Theorem 2.3.4 [SaSc01b] Assume existence of a front solution $q_{*}\left(x-c_{*} t\right)$, $c_{*}>0$, with minimal, critical essential spectrum causing a supercritical Turing instability, as described above.

(I) Assume the Turing instability occurs ahead of the front, at $q_{+}$, only. Then, for each Turing pattern $T(x ; \mu, k)$ with $(k, \mu)$ near $\left(k_{*}, 0\right)$, there exists a modulated front solution of the reaction-diffusion system (2.64), $q(t, x-$ $c(\mu, k) t ; \mu, k)$, invading the Turing pattern

$$
q(t, \xi ; \mu, k) \rightarrow T(\xi+c(\mu, k) t ; \mu, k), \quad \text { for } \xi \rightarrow \infty,
$$

uniformly in time $t$. The modulated front is $\sqrt{\mu}$-close to the primary front $q_{*}$, uniformly in $\xi$ and $t$, and is $P(\mu, k)$-periodic in its first argument, with $P\left(0, k_{*}\right)=2 \pi /\left(c_{*} k_{*}\right)$ and $c\left(0, k_{*}\right)=c_{*}$. See Figure 2.7 for a schematic plot. The bifurcating modulated front is unique in the class of modulated fronts, close to the primary front $q_{*}(\xi)$, invading a fixed Turing pattern, up to spatial and temporal translations.

If the bifurcating Turing pattern is stable, that is, if it lies inside the Eckhaus boundary, then the modulated front is spectrally stable. 
(II) Assume the Turing instability occurs behind the front, at $q_{-}$, only. Then for any parameter value $\mu \sim 0$, any speed $c \sim c_{*}$, any wavenumber $k \sim k_{*}$, and any temporal period $P \sim 2 \pi /\left(c_{*} k_{*}\right)$, there does not exist a modulated front solution $q(t, x-c t)$ to (2.64), which is P-periodic in the first argument, and which leaves a Turing pattern behind

$$
q(t, \xi) \rightarrow T(\xi+c t ; \mu, k), \quad \text { for } \xi \rightarrow-\infty
$$

see Figure 2.8.

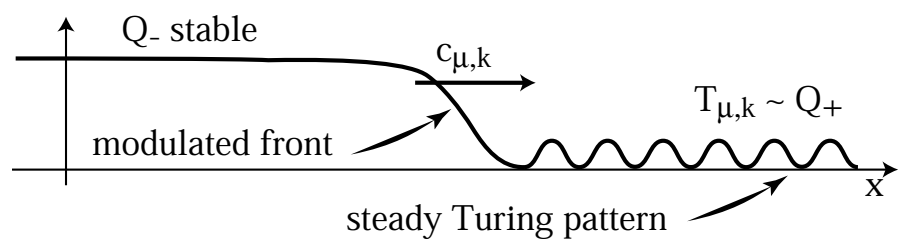

Fig. 2.7. A modulated front invading a Turing pattern, case I

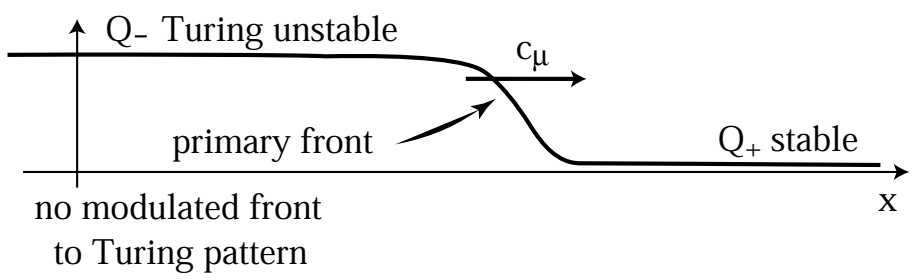

Fig. 2.8. Non-existence of modulated fronts leaving a Turing pattern behind, case II.

Stability in the theorem refers to the spectrum of the temporal period map of the reaction-diffusion system, linearized at the (time-periodic) modulated front in a comoving coordinate frame.

If the front moves to the left, $c_{*}<0$, we may reflect $x$ and find bifurcating modulated waves for an instability at $q_{-}$. In this sense, bifurcation, case I, occurs whenever the front is invading the Turing unstable state, and bifurcation failure, case II, occurs when the front leaves the Turing unstable state behind. 
We emphasize that bifurcation failure, case II, is in contrast to finitedimensional bifurcation theory, where generically periodic solutions arise in Hopf bifurcation.

Instability of a pulse caused by the essential spectrum If $q_{+}=q_{-}=0$, bifurcation is in conflict with bifurcation failure in Theorem 2.3.4, above. The Turing instability then occurs ahead of the front and behind the front, simultaneously. This scenario naturally arises if we consider pulses

$$
0 \not \equiv q_{*}(\xi) \rightarrow 0, \text { for }|\xi| \rightarrow \infty .
$$

Theorem 2.3.5 [SaSc99] Assume existence of a pulse solution $q_{*}\left(x-c_{*} t\right)$, with minimal, critical essential spectrum causing a supercritical Turing instability as described in Section 2.3.2.

Then, for each Turing pattern $T(x ; \mu, k)$ with $(\mu, k) \sim\left(0, k_{*}\right)$, there exists a modulated pulse solution of the reaction-diffusion system (2.64), $q(t, x-$ $c(\mu, k) t ; \mu, k)$, travelling through the Turing pattern. More precisely, there are $(\mu, k)$-independent constants $C, \eta, \eta^{\prime}>0$, and phases $\theta_{ \pm}(\mu, k)$ such that

$$
\begin{aligned}
& \left|q(t, \xi)-T\left(\xi+c t+\theta_{+}\right)\right| \leq C \mathrm{e}^{-\eta|\xi|}, \text { for all } \xi>0, \\
& \left|q(t, \xi)-T\left(\xi+c t+\theta_{-}\right)\right| \leq C \mathrm{e}^{-\eta^{\prime} \mu|\xi|}, \text { for all } \xi<0 .
\end{aligned}
$$

We have suppressed dependence of $q, T, c$, and $\theta_{ \pm}$on $(\mu, k)$. The modulated pulse is $\sqrt{\mu}$-close to the primary pulse, uniformly in $\xi$ and $t$, and periodic in its first argument $t$, with period $P=P(\mu, k), P\left(0, k_{*}\right)=2 \pi /\left(c_{*} k_{*}\right)$ and $c\left(0, k_{*}\right)=c_{*}$. See Figure 2.9 for a schematic plot. The bifurcating modulated pulse is unique in the class of modulated pulses, $(x, t)$-uniformly close to the primary pulse $q_{*}(\xi)$, moving through a fixed Turing pattern, up to spatial and temporal translations.

If the bifurcating Turing pattern is stable, that is, if it lies inside the Eckhaus boundary, then the modulated pulse is spectrally stable.

Again, spectral stability refers to the linearized temporal period- $P$ map along the time-periodic modulated pulse.

Summarizing, we find bifurcation of time-periodic solutions in this case of simultaneous instability ahead and behind a pulse, just like in the case of fronts invading the Turing pattern. Reminiscent of the nonexistence in case of an instability behind the front is the recovery zone of length $1 / \mu$ behind the pulse, marked by the slow convergence to the Turing patterns for $\xi \rightarrow-\infty$.

Fredholm indices and essential instabilities There are several ways of understanding Theorems 2.3.4 and 2.3.5. We first appeal to intuition.

Consider bifurcation of fronts case I, first. Before the instability, $\mu<0$, the homogeneous state $q_{-}$on the left invades the stable state $q_{+}$on the right by means of the front moving with speed $c \sim c_{*}>0$. For $\mu>0$ the homogeneous state ahead of the front has become unstable and Turing 


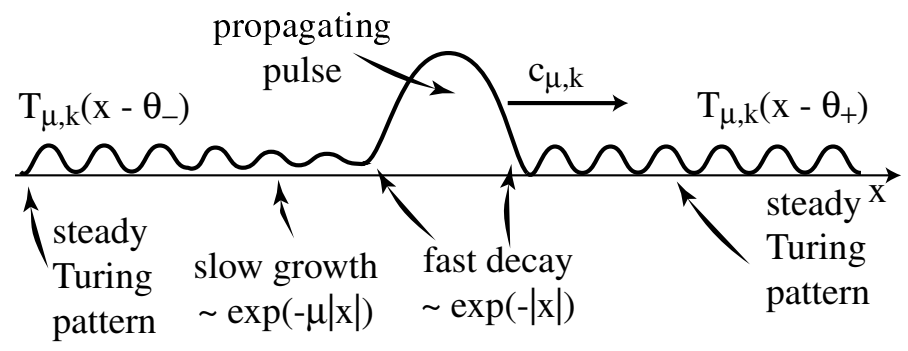

Fig. 2.9. A modulated pulse moving through a Turing pattern

patterns have formed. Intuition suggests that the front would invade these Turing patterns close to the homogeneous state $q_{+}$, which was invaded for $\mu<0$.

Consider bifurcation failure, case II, next, with $\mu>0$. We expect the dynamics near $q_{-}$behind the front to be described approximately by the Ginzburg-Landau equation (2.88). In this approximation, there exist travelling waves, where a Turing pattern invades the unstable homogeneous background $A=0$. For example, we may consider the Turing pattern $A(x) \equiv 1$ constant. The spread of $A \equiv 1$ into the unstable background $A \equiv 0$ (alias $\left.q_{-}\right)$, is then described by the second order travelling wave equation

$$
A^{\prime \prime}+\tilde{c} A^{\prime}+A-A^{3}=0,
$$

for solutions $A=A(\tilde{x}-\tilde{c} \tilde{t})$ of (2.88). Monotone heteroclinic orbits, connecting $A=1$ to $A=0$ exist for all speeds $\tilde{c} \geq 2$. In the unscaled coordinates $t, x$, the speed $\tilde{c}=2$ corresponds to a speed $c_{\text {Turing }}=\mathrm{O}(\sqrt{\mu})$. The heteroclinic represents a small front of a Turing pattern to the left invading the unstable state $q_{-}$, to the right. At present, there is no proof, that Turing patterns actually spread with this (minimal) speed into the unstable homogeneous background state; see, however, [CoEc00] for evidence in this direction. Now observe that in this picture, the speed of a front between a Turing pattern and the homogeneous unstable state $q_{-}$is much slower than the speed of the primary front. Imagine an initial condition consisting of the Turing pattern on the left, followed to the right by the unstable state $q_{-}$, then the front, and finally the stable homogeneous state $q_{+}$on the right. Then the interface between the Turing pattern and the unstable state $q_{-}$moves much slower than the primary front. This leads lead to a wedge opening between the two fronts; see Figure 2.10.

Another explanation would take a functional analytic point of view, still formal, and allude to the results on Fredholm properties of the linearization, Proposition 2.3.1.

Consider case I, first. Observe that the Fredholm index of the linearization $\mathcal{L}_{*}-\mathrm{i} \omega_{*}$ along the front in a comoving frame changes at the bifurcation point 


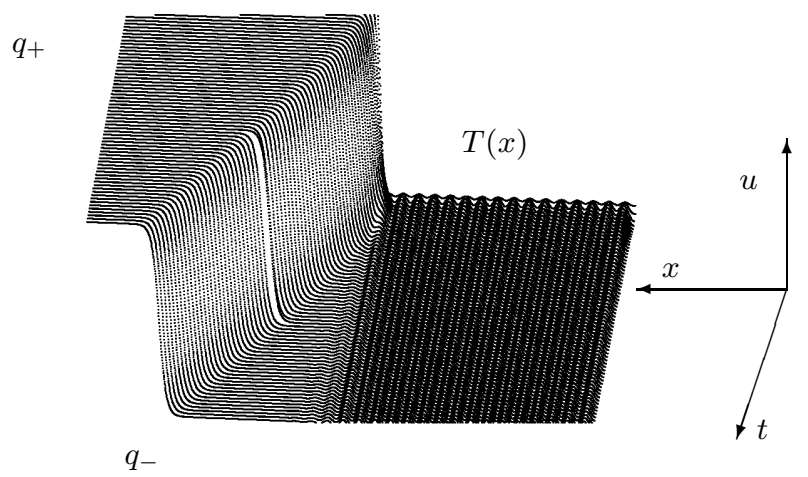

Fig. 2.10. The wedge of an unstable state formed between a small amplitude front of Turing patterns $T(x)$ and a large amplitude front connecting $q_{+}$and $q_{-}$.

$\mu=0$. The group velocity associated with the Turing instability in a steady frame is zero, since $\lambda(\mathrm{i} k)$ is real for all $k \sim k_{*}$. In a comoving frame of speed $c_{*}>0$, the group velocity is negative. Therefore, by Proposition 2.3.1, the Fredholm index changes from 0 to +1 when crossing the critical spectral curve $\lambda(\mathrm{i} k)$ from the unstable to the stable complex half plane; see Figure 2.11.

a)

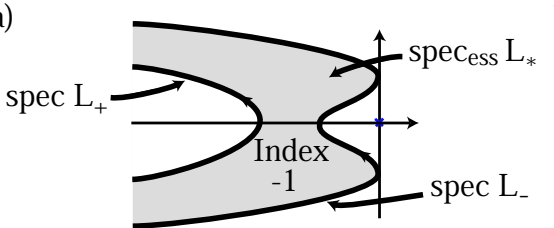

b)

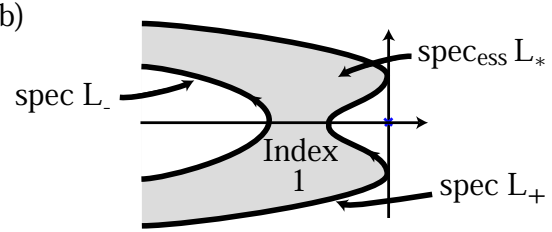

Fig. 2.11. Spectra, Fredholm indices, and oriented critical spectral curves of the linearization about a front, when a Turing instability occurs ahead of the front (a) or behind the front (b).

On the linear level, this index computation suggests that after the instability, $\mu>0$, the Fredholm index of the linearization at $\mathrm{i} \omega_{*}$ is +1 , such that the linearization possesses a one-dimensional kernel, and we should be able to find a family of solutions, parameterized by the wavenumber $k$.

In case II, the analogous argument shows that the Fredholm index is -1 after the instability, $\mu>0$, since, again, the associated group velocity is negative, but now points away from the interface; see Proposition 2.3.1. We therefore do not expect to find any periodic solution since the cokernel is at least one-dimensional. 
However, this reasoning remains formal, since right at the moment of bifurcation, $\mu=0$, the linearization is not Fredholm and Lyapunov-Schmidt reduction does not apply. In fact, the reasoning fails in case of Theorem 2.3.5. The Turing instability occurs simultaneously ahead and behind the front. Therefore, two identical spectral curves touch the imaginary axis simultaneously, both with negative group velocity. One of the curves is associated with the linearization at $q_{+}$, one with $q_{-}$. Again, by Proposition 2.3.1, the Fredholm index to the left and to the right of these two identical critical curves is zero. Still, a family of solutions parameterized by the wavenumber $k$ bifurcates, just like in the case of Fredholm index +1 ; see Theorem 2.3.5.

Spatial dynamics and essential instabilities We now outline our strategy for the proof of Theorems 2.3.4 and 2.3.5. The proofs rely on a spatial dynamics formulation of the parabolic equation (2.66) in the spirit of [Ki82]. Consider the system

$$
\begin{aligned}
U_{\xi} & =V \\
V_{\xi} & =D^{-1}\left(-c V+\omega \partial_{t} U-F(U ; \mu)\right),
\end{aligned}
$$

in a space $(U, V) \in X=H^{1 / 2}\left(S^{1}\right) \times L^{2}\left(S^{1}\right)$ of functions which are $2 \pi$-periodic in time $t \in S^{1}$. The wave speed $c$ and the temporal period $P=2 \pi / \omega$ enter as additional parameters into the equation. We view system (2.89) as an abstract differential equation for $\mathbf{U}=(U, V)(\cdot)$

$$
\mathbf{U}_{\xi}=\mathbf{F}(\mathbf{U} ; \omega, c, \mu) \in X
$$

The goal is to find heteroclinic orbits to this abstract differential equation on a Hilbert space. Indeed, modulated fronts are periodic in time $t$ for an appropriate wave speed $c$. In spatial "time" $\xi$, they converge to the asymptotic states $q_{ \pm}$or the Turing patterns. The Turing patterns are periodic in time $t$ and spatial "time" $\xi$, in the comoving frame. They arise out of a Hopf bifurcation from the equilibrium which undergoes a Turing instability. The essential instability therefore reduces to a global heteroclinic bifurcation, where a heteroclinic orbit connects two equilibria, one of which undergoes a Hopf bifurcation.

Before we proceed to analyze this heteroclinic bifurcation, we want to point out the ill-posed character of (2.90). The initial value problem to this equation is ill-posed, even in the example of the simple heat equation

$$
U_{\xi}=V, \quad V_{\xi}=\partial_{t} U
$$

Indeed, we have solutions $U(\xi, t)=\mathrm{e}^{\mathrm{i} \ell t+\nu_{\ell} \xi}$ to $(2.91)$, with $\nu_{\ell}= \pm \sqrt{\mathrm{i} \ell}$, for any $\ell \in \mathbb{Z}$. In particular, there is no a priori bound on the exponential growth of solutions, neither in forward, nor in backward spatial "time" $\xi$. However, solutions to the initial value problem are unique, provided they 
exist [SaSc01d, Ch98]. The situation is reminiscent of the elliptic boundaryvalue problem $u_{\xi \xi}+u_{y y}=f(u)$, posed on unbounded cylinders $x \in \mathbb{R}$, $y \in(0,1)$; see for example [Ki82, Fi84, Mi94, Fi\&al98].

Still, we can formally linearize (2.90) at equilibria and compute the spectrum of the linearized operator. It then turns out that, just as in the case of the heat equation (2.91), most of the eigenvalues are bounded away from the imaginary axis. It is therefore possible to define stable, unstable, and center manifolds in certain regions of the phase space $X$. In particular, given a solution $\mathbf{U}_{*}(\xi)$ to the nonlinear equation, we can construct stable and unstable manifolds in the vicinity of this solution if the linearized equation

$$
\mathbf{W}_{\xi}=\mathbf{F}^{\prime}\left(\mathbf{U}_{*}(\xi) ; \omega, c, \mu\right) \mathbf{W}
$$

possesses an exponential dichotomy; see [Pe\&al97, SaSc01d] for a definition. Existence of exponential dichotomies on $\mathbb{R}_{+}$is guaranteed, if, $\mathbf{U}_{*}(\xi) \rightarrow \mathbf{U}_{*,+}$ for $\xi \rightarrow \infty$ and the linearized equation about $\mathbf{U}_{*,+}$ does not possess solutions of the form $\mathbf{W}(t) \mathrm{e}^{\mathrm{i} k \xi}$ for any $k \in \mathbb{R}$; see [Pe\&al97, SaSc01d]. Similarly, center, center-unstable, and center-stable manifolds can be constructed, if the weighted linearized equation

$$
\mathbf{W}_{\xi}=\mathbf{F}^{\prime}\left(\mathbf{U}_{*}(\xi) ; \omega, c, \mu\right) \mathbf{W}-\eta_{+} \mathbf{W}
$$

possesses an exponential dichotomy; see again [Pe\&al97, SaSc01d].

The main advantage of our spatial dynamics formulation is that the independent variable $t$ lives on a compact domain, the circle. Time shift provides an $S^{1}$-equivariance in phase space $\mathbf{U} \in X$

$$
\mathbf{U}(\cdot) \mapsto \mathbf{U}(\cdot+\theta) \in X, \quad \theta \in S^{1} ;
$$

see also [Va82] for the role of $S^{1}$-symmetry in Hopf bifurcation. The subspace of functions $(U, V)$ which are fixed under this action of the circle group consists of the time-independent functions. In this subspace, the abstract differential equation (2.90) reduces to the usual travelling wave ODE

$$
U_{\xi}=V, \quad V_{\xi}=D^{-1}(-c V-F(U ; \mu)) .
$$

For $\mu=0$, there is a heteroclinic orbit $\mathbf{U}=Q(\xi)=\left(q(\xi), q^{\prime}(\xi)\right)$ to this differential equation, converging to the "equilibria" $Q_{ \pm}=\left(q_{ \pm}, 0\right)$. Recall again, that the term "equilibria", here, refers to spatial dynamics: "equilibria" are spatially homogeneous solutions.

Consider first the instability ahead of the front, case I in Theorem 2.3.4. We fix the temporal frequency to $\omega_{*}=c_{*} / k_{*}$. The Turing instability turns into a Hopf bifurcation, reflecting spatial periodicity, with (spatial) eigenvalues $\pm \mathrm{i} k_{*}$ from the dispersion relation (2.85). Turing patterns lie in a small neighborhood of $Q_{+}$, but outside of the subspace of time-independent functions. Simplicity of the critical spectral curve, which induces the Turing instability guarantees that the linearization at $Q_{+}$possesses precisely two simple 
imaginary eigenvalues $\nu_{\mathrm{H}}=\mathrm{i} k_{*}$ and $\bar{\nu}_{\mathrm{H}}$, constituting the center eigenspace. After reduction to a 2-dimensional center manifold in a neighborhood of the equilibrium $Q_{+}$, we find a periodic orbit arising through the Hopf bifurcation. Temporal time shift acts nontrivially on the center-manifold such that the periodic orbits actually are relative equilibria, with spatial time evolution given by the temporal time-shift symmetry.

We next argue that the Turing patterns are unstable with respect to the spatial $\xi$-dynamics inside the spatial center manifold. Since $\lambda$ is real in the steady frame, the group velocity associated with the critical spectral curve in the comoving frame is negative; see (2.69) and the following remark. By Lemma 2.3.3, the temporal time-t-instability disappears in a space of functions with weight $\mathrm{e}^{\eta \xi}, \eta>0$ small; see Lemma 2.3.3. In our spatial dynamics picture, this weight shifts the spatial eigenvalues $\nu$ of the linearization at the equilibrium $Q_{+}$, adding $\eta>0$. The effect is that the spatial eigenvalue $\nu_{\mathrm{H}}(\mu)$ stays on the side of the imaginary axis, where it was located for $\mu<0$, suppressing neutral spatial eigenvalues and failure of the Fredholm property throughout the bifurcation. This is only possible, if $\operatorname{Re} \nu_{\mathrm{H}}(\mu)<0$ for $\mu>0$, that is, if the equilibrium $Q_{+}$is stable inside the center manifold, after bifurcation. By exchange of stability, the periodic solution is therefore unstable inside the center manifold.

We turn to the global bifurcation along the heteroclinic orbit $Q(\xi)$, next; compare also Figure 2.12. We argue with the spatial $\xi$-dynamics naively, using objects like (infinite-dimensional) stable and unstable manifolds of the "equilibria" $Q_{ \pm}$freely, as is usual for ODEs; see [SaSc99, SaSc01b] for a technical justification. Just before bifurcation, $\mu<0$, the only bounded solution of equation (2.89), linearized along the front $Q(\xi)$, is $Q^{\prime}(\xi)$. Indeed, any other bounded solution would correspond to a time-periodic solution of the linearized parabolic equation and hence would yield an eigenvalue $2 \pi i \ell / \omega$ of the linearization $\mathcal{L}_{*}$, for some $\ell \in \mathbb{Z}$. Therefore, the intersection of the global stable manifold of $Q_{+}$and the global unstable manifold of $Q_{-}$consists of precisely the heteroclinic $Q(\xi)$. Upon varying $c$, stable and unstable manifold cross with nonzero speed, since the zero eigenvalue was assumed to be simple. This transverse crossing is best viewed in the extended phase space, where the equation (2.89) is augmented with an equation for the parameter, $\mu_{\xi}=0$. In this extended phase space, transverse crossing can be defined as a transverse intersection of the $\mu$-family of stable manifolds with the $\mu$-family of unstable manifolds. Through the bifurcation point $\mu=0$, the stable manifold of $Q_{+}$can be continued smoothly as the strong stable manifold of $Q_{+}$. Indeed, considering the strong stable manifold eliminates the additional weakly stable center direction, which appears at the bifurcation point. Transverse crossing persists as transverse crossing of the strong stable manifold of $Q_{+}$and the unstable manifold of $Q_{-}$, throughout the bifurcation $\mu \sim 0$. The central observation now is that, by continuity of the stable fibration of the center manifold, the strong stable manifold of $Q_{+}$is close to 
any of the strong stable fibers of the periodic orbit in the center manifold! Therefore, the strong stable fibers of the periodic orbit cross the unstable manifold of $Q_{-}$transversely, which yields the desired heteroclinic orbit. If we choose a different strong stable fiber of the periodic orbit, we find a different heteroclinic connection. However, any of those connections can be obtained from the first heteroclinic through the time-t-shift symmetry. Uniqueness of the heteroclinic implies uniqueness of the modulated front in the class of functions with prescribed temporal frequency $\omega$. Varying the temporal time$t$-frequency $\omega$ amounts to a different choice of the wavenumber of the Turing pattern through the relation $k=c / \omega$. In other words, $\omega$ selects the spatial wavenumber of the Turing pattern that we choose to be placed ahead of the front.

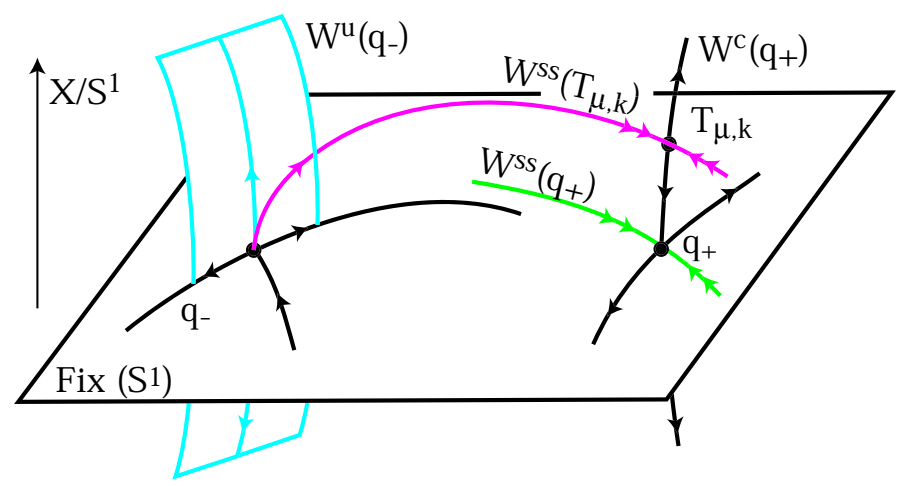

Fig. 2.12. The heteroclinic bifurcation creating a modulated front which invades a Turing pattern. See Plate ?? in the Appendix for a version of this figure in colour.

The picture for the instability behind the front, bifurcation failure case II, is very similar; compare Figure 2.13. The local bifurcation near $Q_{-}$is now the same as the one discussed for $Q_{+}$above. Again, we find a periodic orbit, unstable for the spatial $\xi$-dynamics inside the center-manifold, representing Turing patterns behind the front, in the center manifold close to $Q_{-}$. Before bifurcation, $\mu<0$, the unstable manifold of $Q_{-}$and the stable manifold of $Q_{+}$cross transversely. This time however, we have to continue the unstable manifold of $Q_{-}$through the bifurcation point $\mu=0$. Since the Hopf eigenvalue $\nu_{\mathrm{H}}$ is unstable before the bifurcation, $\mu<0$, the unstable manifold of $Q_{-}$continues smoothly to the center-unstable manifold of $Q_{-}$after bifurcation, $\mu>0$. Transversality yields a unique intersection of the center-unstable manifold of $Q_{-}$with the stable manifold of $Q_{+}$after bifurcation. However, this unique intersection, is already present in the $2 N$-dimensional subspace 
of time-independent solutions: it is given by the trivial continuation of the robust primary front. This proves non-existence of modulated waves, leaving a Turing pattern behind, in the case of an instability behind the front.

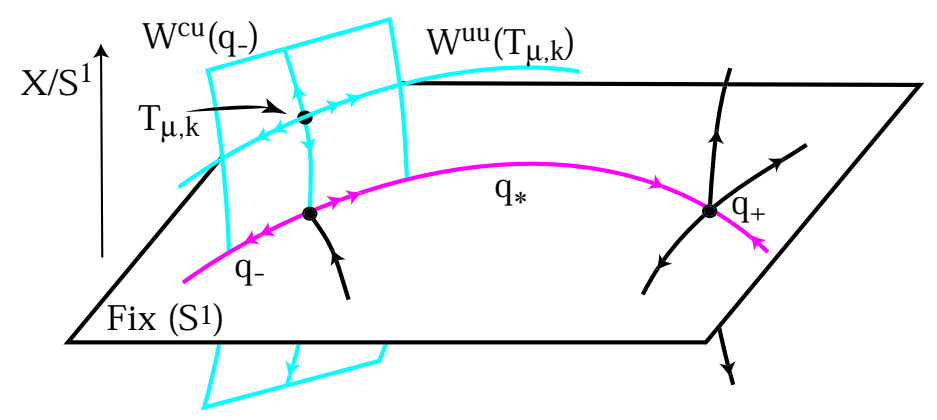

Fig. 2.13. The heteroclinic bifurcation failure of a front leaving a Turing pattern behind. See Plate ?? in the Appendix for a version of this figure in colour.

For a pulse, we combine the two arguments above; see also Figure 2.14. Throughout the bifurcation, the strong stable manifold of the origin crosses the center-unstable manifold of the origin transversely, along $Q(\xi)$. Again by continuity of the strong stable fibration of the center manifold the strong stable fiber of the periodic orbit, representing the Turing pattern ahead of the front, is close to the strong stable manifold of the origin. This implies transverse heteroclinic crossing of this strong stable fiber with the centerunstable manifold of the origin, at an $\mathrm{O}(\mu)$-distance of the periodic orbit. As a consequence, the intersection is not contained in the stable manifold of the origin, which is distance $\mathrm{O}(\sqrt{\mu})$ from the periodic Turing orbit. In backward spatial time $\xi \rightarrow-\infty$, the homoclinic orbit slowly approaches the periodic orbit: it creeps along the center manifold with rate $\mathrm{O}\left(\exp \left(\eta^{\prime} \mu \xi\right)\right)$, as given by the linearization of the periodic orbit inside the center manifold.

For the technically more involved stability considerations, we refer to [SaSc01b, SaSc00b, SaSc01d].

\subsubsection{From Unbounded to Large Domains: Absolute Versus Essential Spectra}

In view of the bifurcation results in Theorems 2.3.4 and 2.3.5, we might try to describe the instability of fronts and pulses in a large, but bounded, comoving domain. Essential spectra disappear since the linearized operator $\mathcal{L}_{*}$ from 


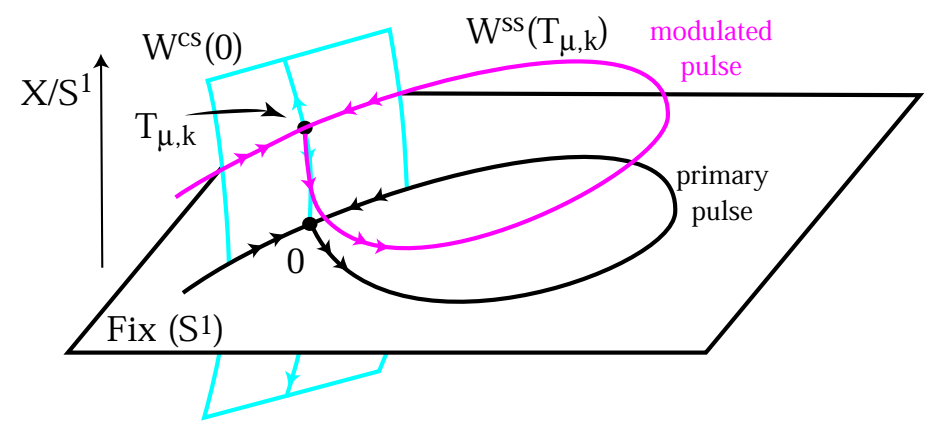

Fig. 2.14. The homoclinic bifurcation creating a modulated pulse travelling through a Turing pattern. See Plate ?? in the Appendix for a version of this figure in colour.

(2.66) possesses a compact resolvent on any finite domain. Therefore any instability in a bounded domain is necessarily due to point spectrum crossing the imaginary axis. In case of a pair of complex conjugate eigenvalues crossing the axis, a general Hopf bifurcation theorem guarantees bifurcation of periodic orbits - under mild nondegeneracy conditions. In consequence, there is an obvious difficulty trying to approximate instabilities in unbounded domains by instabilities in bounded domains. In particular the bifurcation failure in case II of Theorem 2.3.4, cannot be reproduced in any approximating bounded domain.

For a better understanding of this difficulty, we return to the simple example of the advection-diffusion problem

$$
w_{t}=w_{\xi \xi}+w_{\xi}+\mu w=: \mathcal{L}_{\mu} w .
$$

Compared to (2.75), we have added a constant linear driving term $\mu w$. In the unbounded domain $x \in \mathbb{R}$, the instability threshold is $\mu=0$, where the essential spectrum,

$$
\operatorname{spec}_{\text {ess }} \mathcal{L}_{\mu}=\left\{\lambda=-k^{2}-\mathrm{i} k+\mu ; k \in \mathbb{R}\right\},
$$

crosses the imaginary axis.

Truncating to $x \in(-L, L)$ and imposing periodic boundary conditions causes the curves of essential spectra to break up into point spectrum. The eigenvalues are still located in a neighborhood of the curve of essential spectrum, and can be computed explicitly:

$$
\operatorname{spec}_{\text {per },(-L, L)} \mathcal{L}_{\mu}=\left\{\lambda_{\ell}=-\left(\frac{\pi \ell}{2 L}\right)^{2}+\mathrm{i} \frac{\pi \ell}{2 L}+\mu ; \ell \in \mathbb{Z}\right\} .
$$


On bounded subsets of the complex plane, $\operatorname{spec}_{\text {per, }(-L, L)} \mathcal{L}_{\mu}$ converges to the essential spectrum $\operatorname{spec}_{\text {ess }} \mathcal{L}_{\mu}$, in the symmetric Hausdorff distance, for $L \rightarrow \infty$.

Imposing separated boundary conditions, for example Dirichlet boundary conditions $u(-L)=u(L)=0$, the spectrum of $\mathcal{L}_{\mu}$ again consists of point spectrum. However, the point spectrum is now located on the real axis, with eigenvalues given explicitly by

$$
\operatorname{spec}_{\operatorname{Dir},(-L, L)} \mathcal{L}_{\mu}=\left\{\lambda_{\ell}=-\left(\frac{\pi \ell}{2 L}\right)^{2}+\mu-\frac{1}{4} ; \ell \in \mathbb{Z}\right\} .
$$

The eigenfunctions are exponentially localized at the left boundary of the interval $(-L, L)$. On bounded subsets of the complex plane, the point spectrum converges to $\left(-\infty, \mu-\frac{1}{4}\right]$ in the symmetric Hausdorff distance, for $L \rightarrow \infty$. We will later identify this half line as the absolute spectrum of the operator $\mathcal{L}_{\mu}$, posed on $x \in \mathbb{R}$.

Note that the half line $\lambda \leq \mu-1 / 4$ is not part of the spectrum of $\mathcal{L}_{\mu}$ on $x \in \mathbb{R}$, but merely arises as a set-wise limit of the spectrum if we truncate to finite intervals and let $L$ tend to infinity. It is not difficult, but tedious, to check that almost all separated mixed boundary conditions induce a similar limiting behavior of the spectrum on large intervals $(-L, L)$, as the length of the interval tends to infinity. For example, in the case of Neumann boundary conditions, the spectrum converges

$$
\operatorname{spec}_{(-L, L), \text { sep }} \mathcal{L}_{\mu} \longrightarrow\left(-\infty, \mu-\frac{1}{4}\right] \cup\{\mu\} \quad \text { for } L \rightarrow \infty,
$$

on bounded subsets of the complex plane $\mathbb{C}$, in the symmetric Hausdorff distance. It is an interesting exercise to follow the eigenvalue $\lambda=\mu$ through a homotopy from Neumann to Dirichlet through mixed boundary conditions.

For Dirichlet boundary conditions and $0<\mu<\frac{1}{4}$, the system is unstable on the real axis $x \in \mathbb{R}$, whereas it is stable under separated boundary conditions, for any size of the domain. In this parameter regime, we call the system convectively unstable. Indeed, the instability on the unbounded domain is convective in nature: starting with initial conditions $u_{0}(x)$ having compact support on $\mathbb{R}$, the absolute value $\left|u\left(t, x_{0}\right)\right|$ converges to zero for every fixed point $x_{0}$ on the real axis, although the norm $\|u(t, \cdot)\|_{L^{2}}$ grows exponentially. The pointwise decay can be extracted from the explicit representation of solutions by the Green's function for (2.93).

This general phenomenon of pointwise decay of solutions, which nevertheless grow in norm, was first emphasized in the context of plasma physics and fluid flow instability [LaLi59, Br64]. We will now study the behavior of spectra of pulses or fronts in reaction-diffusion systems, in the limit $L \rightarrow \infty$; see $[\mathrm{SaSc00c}]$ for a more general result.

Consider the linear reaction-advection-diffusion system

$$
\mathcal{L} u=D u_{\xi \xi}+c u_{\xi}+a(\xi) u,
$$


where the $N \times N$-matrix $a(\xi)$ converges exponentially to $a_{ \pm}$for $\xi \rightarrow \pm \infty$. At $\xi= \pm \infty$, we encounter the asymptotic linearized operators

$$
\mathcal{L}_{ \pm} u=D u_{\xi \xi}+c u_{\xi}+a_{ \pm} u
$$

Associated with $\mathcal{L}_{ \pm}$are the dispersion relations

$$
d_{ \pm}(\lambda, \nu)=\operatorname{det}\left(D \nu^{2}-c \nu+a_{ \pm}-\lambda\right)=0,
$$

which are polynomials of degree $2 N$ in $\nu$. Let $\nu_{ \pm}^{j}(\lambda), j=1, \ldots, 2 N$, denote the complex roots, ordered by decreasing real part

$$
\operatorname{Re} \nu_{+}^{1} \geq \ldots \geq \operatorname{Re} \nu_{+}^{2 N}, \quad \operatorname{Re} \nu_{-}^{1} \geq \ldots \geq \operatorname{Re} \nu_{-}^{2 N} .
$$

Note that this numbering might depend discontinuously on the spectral parameter $\lambda$.

In the homoclinic case $a_{+}=a_{-}$, the essential spectrum, $\operatorname{spec}_{\text {ess }}(\mathcal{L})$, is given by the set of $\lambda$ such that $\operatorname{Re} \nu_{+}^{j}(\lambda)=0$ or $\operatorname{Re} \nu_{-}^{j}(\lambda)=0$, for some $j$; see Proposition 2.3.1.

In the general case of a pulse or a front, we define the absolute spectrum, $\operatorname{spec}_{\text {abs }}(\mathcal{L})$, as the set of $\lambda \in \mathbb{C}$ such that $\operatorname{Re} \nu_{+}^{N}(\lambda)=\operatorname{Re} \nu_{+}^{N+1}(\lambda)$ or $\operatorname{Re} \nu_{-}^{N}(\lambda)=\operatorname{Re} \nu_{-}^{N+1}(\lambda)$. Note that the absolute spectrum is not necessarily part of the essential spectrum. Still, from this definition, the absolute spectrum is defined by the operator posed on the unbounded domain $\xi \in \mathbb{R}$. The reader is invited to check that the absolute spectrum of the advectiondiffusion problem (2.94) is actually given by the half line $\left(-\infty, \mu-\frac{1}{4}\right]$. In general, the absolute spectrum is a closed set and consists of a finite collection of curves.

An important property of the absolute spectrum is that in its complement, there exist exponential weights $\underline{\eta}(\lambda)=\left(\eta_{-}(\lambda), \eta_{+}(\lambda)\right) \in \mathbb{R}^{2}$ such that $\mathcal{L}-\lambda$ is Fredholm with index zero when considered as a closed operator on $L_{\underline{\eta}(\lambda)}^{2}$, the space of functions in $L_{\mathrm{loc}}^{2}$ with

$$
\|u\|_{L_{\underline{\underline{n}}}^{2}}^{2}:=\int_{\mathbb{R}^{+}}\left|\mathrm{e}^{\eta_{+} \xi} u(\xi)\right|^{2}+\int_{\mathbb{R}^{-}}\left|\mathrm{e}^{\eta_{-} \xi} u(\xi)\right|^{2}<\infty .
$$

In Theorem 2.3.6 below we characterize the limiting behavior of spectra, as the domain size tends to infinity. We will consider periodic boundary conditions, $u(-L)=u(L), u_{\xi}(-L)=u_{\xi}(L)$, as well as separated boundary conditions, $\left(u( \pm L), u_{\xi}( \pm L)\right) \in E_{ \pm}$with given, $N$-dimensional subspaces $E_{ \pm}$of $\mathbb{C}^{2 N}$. Periodic boundary conditions are understood with the restriction $a_{+}=a_{-}$, that is, we are interested in linearizations about pulses.

The point spectrum, $\operatorname{spec}_{\mathrm{pt}}(\mathcal{L})$, refers to the point spectrum in $L^{2}(\mathbb{R})$. For the case of separated boundary conditions, we distinguish two types of point spectrum. Fix $\lambda_{*} \notin \operatorname{spec}_{\text {abs }}(\mathcal{L})$, and the weight $\left(\eta_{-}^{*}, \eta_{+}^{*}\right)=\underline{\eta}_{*}=\underline{\eta}_{*}\left(\lambda_{*}\right)$ such that $\mathcal{L}$ is Fredholm of index zero for $\lambda$ near $\lambda_{*}$ in $L_{\underline{\eta}_{*}}^{2}$. We say $\lambda_{*}^{*}$ belongs to the extended point spectrum $\operatorname{spec}_{\text {expt }}(\mathcal{L})$, if $\mathcal{L}-\bar{\lambda}^{*}$ is not invertible 
in $L_{\underline{\eta}_{*}}^{2}$. We say $\lambda_{*}$ belongs to the right boundary spectrum, $\operatorname{spec}_{\mathrm{bdy},>}(\mathcal{L})$, if the asymptotic, constant coefficient operator $\mathcal{L}_{+}-\lambda_{*}$ possesses nontrivial kernel when considered on $\xi>0$, in $L_{\eta_{+}^{*}}^{2}\left(\mathbb{R}_{+}\right)$with boundary condition $E_{+}$ in $\xi=0$. Similarly, the left boundary spectrum is defined via the kernel of $\mathcal{L}_{-}-\lambda_{*}$ in $L_{\eta_{-}^{*}}^{2}\left(\mathbb{R}_{-}\right)$with boundary condition $E_{-}$in $\xi=0$. The boundary spectrum spec $_{\text {bdy }} \mathcal{L}$ is defined as the union of left and right boundary spectra. We refer to [SaSc00c] for a more geometric and constructive definition of boundary spectra, involving winding numbers or, alternatively, Evans function constructions.

Several assumptions are needed in the proof of the following theorem. Most of them are technical in nature and satisfied for "typical" systems. We therefore refer the reader to [SaSc00c] for precise hypotheses.

Theorem 2.3.6 [SaSc00c] Under typical assumptions on the spectrum on the real line, we find that the spectrum of $\mathcal{L}$ on $L^{2}(-L, L)$ converges, as $L \rightarrow \infty$ :

- under periodic boundary conditions,

$$
\operatorname{spec} \mathcal{L}_{\text {per },(-L, L)} \longrightarrow \operatorname{spec}_{\text {ess }}(\mathcal{L}) \cup \operatorname{spec}_{\text {pt }}(\mathcal{L}) ;
$$

- under typical, separated boundary conditions,

$$
\operatorname{spec} \mathcal{L}_{\text {sep },(-L, L)} \longrightarrow \operatorname{spec}_{\text {abs }}(\mathcal{L}) \cup \operatorname{spec}_{\text {expt }}(\mathcal{L}) \cup \operatorname{spec}_{\text {bdy }}(\mathcal{L}) .
$$

Convergence is understood on bounded subsets of the complex plane in the symmetric Hausdorff distance.

Moreover, multiplicity of the eigenvalues is preserved. The number of eigenvalues, counted with multiplicity, in any fixed open neighborhood of a point $\lambda_{*} \in \operatorname{spec}_{\text {ess }}(\mathcal{L})$ (or $\operatorname{spec}_{\text {abs }}(\mathcal{L})$, respectively) converges to infinity as $L \rightarrow \infty$. Multiplicities in neighborhoods of points $\lambda_{*} \in \operatorname{spec}_{\text {expt }}(\mathcal{L}), \operatorname{spec}_{\mathrm{pt}}(\mathcal{L})$, $\operatorname{spec}_{\mathrm{bdy}}(\mathcal{L})$ stabilize and convergence of eigenvalues is of exponential rate in $L$, there.

The most important, and at first sight surprising, part of the theorem is that spectra on large intervals with separated boundary conditions do not approximate essential, but merely absolute spectra. The latter are in general different from the essential spectrum. However, a reflection symmetry $x \rightarrow-x$ in the problem can force both spectra to coincide.

The theorem shows that the limit of spectra on bounded intervals is entirely determined by "spectral" information from the unbounded domain limit, together with information on the boundary conditions.

Let us return to the bifurcation problem in Theorem 2.3.4. An intriguing question is, what happens to the instability when restricting the domain to a bounded interval in an appropriately comoving frame. This restriction becomes necessary, for example, when trying to detect instabilities of fronts 
numerically. The critical spectrum in Theorem 2.3.4 consists of an isolated zero eigenvalue and of essential spectrum with non-zero group velocity. With a positive exponential weight $\exp (\eta \xi)$ with $\eta>0$, on $\xi \in \mathbb{R}$, we can shift the essential spectrum into the stable complex half plane. By Theorem 2.3.6, the only critical eigenvalue in a large domain is going to be the zero eigenvalue. Its precise location depends on the actual choice of boundary conditions. In particular, there is no Hopf bifurcation in any, arbitrarily large but finite, domain caused by the essential spectrum. The only possible instability mechanism would be the creation of unstable spectrum through the boundary conditions, in $\operatorname{spec}_{\mathrm{bdy}} \mathcal{L}$.

Linearized stability, Re $\operatorname{spec} \mathcal{L}_{*}<0$, in large bounded domains $(-L, L)$ implies nonlinear stability, for reaction-diffusion systems. However, our above result on the spectrum for $L \rightarrow \infty$ makes extensive use of exponential weights, which might be incompatible with the nonlinearity. For example, with $\eta:=$ $\eta_{+}=\eta_{-}>0$, the nonlinearity $u \mapsto u^{2}$ is not defined on $H_{\eta}^{1}$, the space of functions $u$ with $u, u^{\prime} \in L_{\eta}^{2}$. This technical observation is closely related to limitations of the above theorem. For an illustration, let us return to

$$
u_{t}=u_{x x}+u_{x}+\mu u+\kappa u^{3}, \quad u(-L)=u(L)=0
$$

with $L$ large. For $0<\mu<1 / 4$, the absolute spectrum is contained in the open left half plane and $u(x) \equiv 0$ is asymptotically stable. Define the basin of attraction $B_{L}$ as the set of initial values $u_{0} \in H_{\text {Dir }}^{1}(-L, L)$ such that the solution of $(2.95)$ with initial value $u_{0}$ converges to zero. As a measure for stability, we define the instability threshold

$$
\delta^{\mathrm{u}}(L)=\inf \left\{\left\|u_{0}\right\|_{H^{1}} ; u_{0} \notin B_{L}\right\} .
$$

The instability threshold measures the minimal amount of a perturbation needed to "permanently" drive the system away from the equilibrium. The smaller $\delta^{\mathrm{u}}(L)$, the more sensitive the equilibrium $u \equiv 0$ will be to perturbations.

Proposition 2.3.7 [SaSc02a] Consider equation (2.95) with $0<\mu<1 / 4$. Then the instability threshold $\delta^{\mathrm{u}}(L)$ satisfies

$\left(a \Phi^{\mathrm{u}}(L) \geq \delta_{0}>0\right.$ if $\kappa<0$, for all $L>0$;

$\left(b \phi^{\mathrm{u}}(L)=\mathrm{O}(1 / L)\right.$ if $\kappa>0$.

In particular, for the unstable sign (b) of the nonlinearity $\kappa>0$, the stability predicted from the spectral analysis, with uniform exponential decay rate $\mu-1 / 4$, is valid only in a very small region of phase space, if the size $L$ of the domain is large. Even small initial conditions may then lead to blow-up in finite time. We do not know if the upper estimate $\mathrm{O}(1 / L)$ on the instability threshold is optimal in case (b). 


\subsection{Two Space Dimensions: Existence of Spiral Waves}

The spontaneous appearance of rotating spiral-shaped planar patterns is a most striking phenomenon, both from the experimental and the theoretical point of view. Spiral wave patterns have been observed for example in heart tissue [WiRo46], in Belousov-Zhabotinsky (BZ) reactive media [Wi72, BrEn93, Un\&al93], in the CIMA-reaction [dK\&al94], in fluid convection experiments [PlBo96], and in surface catalysis [Ne\&al]. From a mathematical view point, they pose challenges because they arise in large - ideally unbounded — domains; see Section 2.4.2.

Pioneering work by Wiener, 1946, has been motivated by waves of electrical excitation in heart muscle tissue. It is well worth obtaining the somewhat obscure original reference [WiRo46]. See also the overwhelming material collected by [Wi01, Wi87], and the references there. One approach initiated by Wiener aims at a direct geometric description of the spatio-temporal dynamics of the appearing sharp wave fronts, which far from a core region take the form of Archimedean spirals. This approach is sometimes called "kinematic theory of spirals", and has been developed further on a mostly formal level; see for example [KeTy92, Ke92, MiZy91] and the references there. The underlying idea is closely related to the problem of "curve shortening", which has seen significant recent progress; see [An90, An91, GaHa86].

Spiral waves appear both in excitable and oscillatory media. In the latter, the interpretation changes from a source of excitation waves to a defect in the pattern of phase waves emitted by the spiral core. At the center, or core, the oscillation phase cannot be defined in a continuous way. Along a simple closed path around the core, the phase of the oscillations winds around the circle once. Small-amplitude oscillations are amenable to the powerful tool of modulation equations such as the complex Ginzburg-Landau equation or $\lambda$ - $\omega$-systems; see [KoHo73, Ha82, KoHo81].

In the following two sections, we expose two simple approaches to this problem. We first address excitable media and a kinematic description of the spiral arms in Section 2.4.1.

In Section 2.4.2, we then characterize spiral wave solution to reactiondiffusion systems more generally, aiming at a mathematical definition which incorporates the characteristic features of experimentally observed spirals. The formulation in this section will be the basis for the spectral discussion in Section 2.5.3.

Small-amplitude spiral waves arising near Hopf bifurcations in the reaction kinetics then provide us with specific examples, in Section 2.4.3.

\subsubsection{Kinematics and its Defects}

To state the problem in its most simplistic and, as we will see, yet inadequate form, we consider a differentiable planar curve $s \mapsto(r, \varphi)(s)$ in polar coordinates $(r, \varphi)$. Arc length is denoted by $s$. We assume that the curve propagates 
in time, with scalar normal velocity $c_{\perp}$ which depends on the signed local curvature $\kappa$ in a strictly monotone way. (We fix the normal direction to the left of the tangent vector here.) For later convenience, we write

$$
c_{\perp}=\mathrm{v}^{-1}(\kappa)
$$

to denote the inverse dependence. Let $\theta \in S^{1}$ denote the angle which the unit tangent to our curve forms with the horizontal axis; see Figure 2.15. Denoting the derivative of $\theta$ with respect to arc length $s$ by $\dot{\theta}$, we obtain $\dot{\theta}=\kappa$ and, by $(2.96)$,

$$
\dot{\theta}=\mathrm{v}\left(c_{\perp}\right)
$$

If the curve is to rotate left at constant angular velocity $\omega$ with respect to the center $r=0$ of our polar coordinate system $(r, \varphi)$, then

$$
c_{\perp}=\omega r \cos (\theta-\varphi) .
$$

Combining (2.97), (2.98) with the definition of arc length parameterization we obtain the ODE system

$$
\begin{aligned}
& \dot{\theta}=\mathrm{v}(\omega r \cos (\theta-\varphi)) \\
& \dot{\varphi}=r^{-1} \sin (\theta-\varphi) \\
& \dot{r}=\cos (\theta-\varphi)
\end{aligned}
$$

for any rigidly rotating curve $(r(s), \varphi(s), \theta(s))$ under the geometric equation (2.96).

Curve shortening as mentioned above corresponds to the special case $\mathrm{v}\left(c_{\perp}\right)=\kappa$ of normal velocity proportional to $\kappa$. As for all $\mathrm{v}$ of positive slope, a Sturm property analogous to Proposition 2.2.1 holds here: the number of strict crossings of two distinct curves never increases under this curvature flow. We note here that positive $\mathrm{v}$-slope, $\mathrm{v}^{\prime}>0$, corresponds to a nonlinear forward parabolic equation for the curve evolution; see [An91]. The existence of rotating wave "Ying-Yang" spiral curves seems to be folklore in the subject. The resulting spirals, however, are not Archimedean: they limit from inside onto a circle of finite radius, instead. Negative slope, in contrast, would correspond to a backwards parabolic equation which is ill-posed in forward time. Although this may suggest an incompleteness in the model derivation of the curve-"lengthening" equation, it does not cause difficulties for our rotating wave ODE (2.99). Variants involving a power dependence like $\mathrm{v}^{-1}(\kappa)=\kappa^{\beta}$ have also been considered: most notably the affine invariant case $\beta=1 / 3$ for self-focusing edge enhancement in image processing. For propagation of Belousov-Zhabotinsky (BZ) fronts, a good model is generally believed to be given by

$$
\mathrm{v}^{-1}(\kappa)=c_{0}+\kappa
$$

on suitable time scales, see [KeTy92, MiZy91] and the references there. 
Curvature flow of Archimedean spirals Let us look for Archimedean spirals in system (2.99), as are observed in BZ media. We consider Archimedean spirals to be characterized by an asymptotically proportional dependence of radius $r$ on angle $\varphi$, given by a limiting slope

$$
\sigma_{*}:=\lim _{r \rightarrow \infty} \frac{d r}{d \varphi}=\lim _{s \rightarrow \infty} \frac{r \cos \alpha}{\sin \alpha} \notin\{0, \pm \infty\} .
$$

Here we have used l'Hospital in (2.99), with the abbreviation

$$
\alpha:=\theta-\varphi
$$

Our curve evolution is in fact rotation invariant, as is $\alpha=\theta-\varphi$ but neither $\theta$ nor $\varphi$ individually. It is therefore convenient to rewrite (2.99) in terms of $(r, \alpha)$ :

$$
\begin{aligned}
\dot{\alpha} & =\mathrm{v}(\omega r \cos \alpha)-r^{-1} \sin \alpha \\
\dot{r} & =\cos \alpha .
\end{aligned}
$$

From (2.101) in the limit $s \rightarrow \infty$, alias $r \rightarrow \infty$, we immediately deduce that $\cos \alpha \rightarrow 0$ and in fact

$$
\begin{aligned}
\alpha & \rightarrow \pm \pi / 2 \\
\sigma:=r \cos \alpha & \rightarrow \pm \sigma_{*} .
\end{aligned}
$$

with corresponding signs \pm for either limit. To analyze these limits further, we rewrite (2.103) in terms of $r$ and $\rho:=1 / r \rightarrow 0$ as follows

$$
\begin{aligned}
\sigma^{\prime} & = \pm \sqrt{1-\rho^{2} \sigma^{2}} \mathrm{v}(\omega \sigma)+\rho \\
\rho^{\prime} & =-\rho^{4} \sigma
\end{aligned}
$$

Here we have rescaled "time" $s$ such that $\rho \mathrm{d} / \mathrm{d} s={ }^{\prime}$. The vector field (2.105) is regular along the invariant line $\rho=0$, where

$$
\sigma^{\prime}= \pm \mathrm{v}(\omega \sigma) \text {. }
$$

The limit slope (2.101) therefore requires the equilibrium condition

$$
0=\mathrm{v}\left(\omega \sigma_{*}\right)
$$

to hold. This condition amounts to a dispersion relation between the rotation frequency $\omega$ and the wave length $2 \pi \sigma_{*}$ of the asymptotically uncurved, planar fronts of our Archimedean spirals. For example $\sigma_{*}=-c_{0} / \omega$ in the BZ case mentioned above.

A center manifold analysis at the Archimedean spiral equilibrium $\rho=$ $0, \sigma=\sigma_{*}$ of (2.105) yields additional information on those Archimedean spiral trajectories. Consider the case $\alpha \rightarrow+\pi / 2, \sigma_{*}>0$ of left outward winding spirals, first. Then $\sigma=\sigma_{*}$ is hyperbolically stable, within the line $\rho=0$, if 


$$
\omega \mathrm{v}^{\prime}\left(\omega \sigma_{*}\right)<0 .
$$

Since $\rho \searrow 0$ inside the center manifold, any initial condition of (2.105) with $\rho>0$ and $(\rho, \sigma)$ near $\left(0, \sigma_{*}\right)$ then converges to this Archimedean spiral equilibrium. Most notably this includes the BZ example, where $\mathrm{v}^{\prime}>0, \sigma^{*}>0$, and $\omega<0$. The opposite case $\omega \mathrm{v}^{\prime}\left(\omega \sigma^{*}\right)>0$ arises for example when curves shorten due to $\mathrm{v}^{\prime}>0, c_{0}<0<\omega$. Then $\sigma=\sigma_{*}$ is hyperbolically unstable, within the line $\rho=0$, and the asymptotically Archimedean spiral is uniquely defined. In either case, the center manifold - be it unique or not - provides convergence to the Archimedean spiral equilibrium along the same asymptote

$$
\sigma=\sigma(r) \sim \sigma_{*}-\left(\omega \mathrm{v}^{\prime}\left(\omega \sigma_{*}\right)\right)^{-1} r^{-1}+O\left(r^{-2}\right) .
$$

The $r^{-1}$-term measures the deviation from a precise Archimedean spiral. Similarly, polar coordinate asymptotics for $r=r(\varphi)$ can be derived.

The front-back matching problem The two sign-cases in (2.105) are related by a simple transformation. Indeed $(\rho, \sigma, s) \mapsto(-\rho, \sigma, s)$ reverses the sign \pm as well as the sign of ${ }^{\prime}$. This means that the unique equilibrium $\sigma=\sigma_{*}$ on the line $\rho=0$ possesses the same stability properties under the $s$-flow, in either case. In particular it is impossible to find a rotating wave solution as in Figure 2.15(a), which would be biasymptotic to an Archimedean spiral for both $s \rightarrow+\infty$ and $s \rightarrow-\infty$.

This impossibility indicates a first defect of our naive, inadequate approach based on motion by curvature (2.96) alone. Two appealing remedies have been proposed. The first derives curvature motion for fronts $(s \rightarrow+\infty)$ and backs $(s \rightarrow-\infty)$ separately, by singular perturbation arguments, and then attempts a formal matching in the "core" region where the arc length parameter $|s|$ is small. See for example [Ke92].
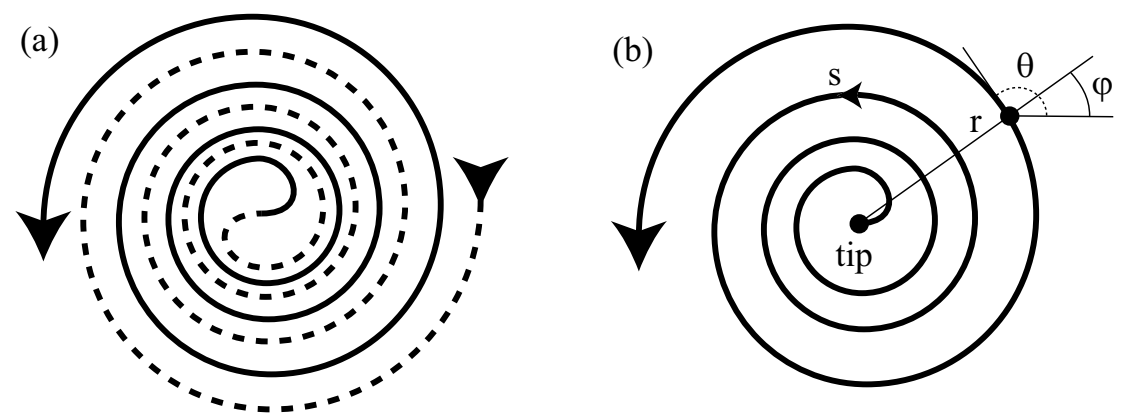

Fig. 2.15. Biasymptotic Archimedean spiral (a), and spiral with spiral tip (b).

More radically the second remedy terminates the spiral at $s=0$ and proposes additional phenomenological equations of motion at this "tip" point, 
see for example [MiZy91], and Figure 2.15(b). Neither approach has been fully justified on a satisfactory mathematical basis, so far. For scalar equations, $u \in \mathbb{R}$, the singular perturbation limit $\varepsilon \searrow 0$ for reaction-diffusion equations

$$
\varepsilon u_{t}=\varepsilon^{2} \Delta u+f(u)
$$

has been analyzed successfully for bounded domains $x \in \Omega \subset \mathbb{R}^{N}$, and (mostly) cubic nonlinearities

$$
f(u)=(u-a)\left(1-u^{2}\right) .
$$

Concerning planar wave fronts, alias $N=1$, between the stable equilibria $u \equiv \pm 1$ we have already mentioned the travelling waves $u=u\left(\left(x-c_{0} t\right) / \varepsilon\right)$ of wave speed $c_{0}=c_{0}(a)$. Note that $c_{0}(-a)=-c_{0}(a)$, by the symmetry $u \mapsto-u$. Based on earlier formal expansions by Fife, an asymptotics

$$
c_{\perp}=c_{0}(a)+\varepsilon \kappa+\cdots
$$

has been proved to hold for $N=\operatorname{dim} x=2$, over time spans $0 \leq t \leq T / \varepsilon$, for the interface curves of solutions $u=u^{\varepsilon}$ of (2.110). Mean curvature flow for $(N-1)$-dimensional interfaces has also been justified, for $N \geq 3$. See [Fi88, Ba\&al93] and the references there.

Expansion (2.112) fits well with the BZ-Ansatz (2.100) for $c_{\perp}=\mathrm{v}^{-1}(\kappa)$. Choosing $a=a(\varepsilon)=\varepsilon a_{1}$ we can in fact scale $\varepsilon$ out of (2.112) by rescaling time, because oddness of $c_{0}(a)$ then implies $c_{0}(a)=\varepsilon c_{0}^{\prime}(0) a_{1}+\cdots$. Then (2.112) takes precisely the form (2.100) discussed above.

However, rigorous analysis of (2.110) is heavily based on variational methods and hence fails, as it stands, in two important cases: in unbounded domains $\Omega$ because the Lyapunov functional $\mathcal{V}=\mathcal{V}_{\varepsilon}$ of (2.4) may become unbounded, and for systems $u \in \mathbb{R}^{2}$ because $\mathcal{V}$ may not exist.

The front-back difficulty, which causes the inexistence of biasymptotic Archimedean spirals as in Figure 2.15(a), already arises in one space dimension $N=1$ and is due to the planar front wave speeds $c_{0}(a)$ in (2.100), (2.112). Indeed, adjacent curve segments in Figure 2.15(a) correspond to limits $s \rightarrow \pm \infty$, alternatingly, where curvature $\kappa$ is negligible and

$$
c_{\perp} \approx c_{0}(a)
$$

approaches the planar wave front limit. But the normal directions point in opposite directions, for adjacent segments. Alternatively, with respect to a fixed normal direction or as is appropriate in one space dimension $N=1$, we can invoke $c_{0}(-a)=-c_{0}(a)$ and observe that adjacent fronts and backs move in opposite directions, unless $c_{0}(a)=0$. That latter possibility is excluded, both by experimental observation and by our constitutive assumption $\sigma_{*} \neq 0$ for Archimedean spirals, in (2.101).

As an alternative to this dilemma we might feel tempted to consider travelling pulses, for $N=1$, instead of fronts and backs. Rescaling $u=$ 
$u\left(\left(x-c_{0} t\right) / \varepsilon\right)$ by $\varepsilon \searrow 0$, the pulse locations would contract to curves, for $N=2$, which could move according to curvature. For scalar equations, $u \in \mathbb{R}$ however, this is prevented by the (damped) Hamiltonian structure of the travelling wave equation, which forces vanishing wave speed $c_{0}=0$ for any homoclinic pulse. Placing a single pulse on any large circle $r=$ const., moreover, the curve of pulse locations would have to terminate at a "tip" as in Figure 2.15(b).

Clearly this Hamiltonian difficulty disappears for non-variational systems $u \in \mathbb{R}^{2}$. We conclude with a standard example for excitable media systems: the FitzHugh-Nagumo system

$$
\begin{aligned}
& u_{t}^{1}=\Delta u^{1}+\varepsilon^{-1} u^{1}\left(1-u^{1}\right)\left(u^{1}-\left(u^{2}+b\right) / a\right) \\
& u_{t}^{2}=d \Delta u^{2}+u^{1}-u^{2} .
\end{aligned}
$$

For given $u^{2}=u^{2}(t, x)$, the singularly perturbed $u^{1}$-equation is cubic as discussed above, and $u^{2}$ enters as a parameter. Adjusting the constants $a, b$ appropriately, homoclinic pulses arise which travel at nonzero speeds. This case is usually termed excitable medium; see Figure 2.16(a).

(a)

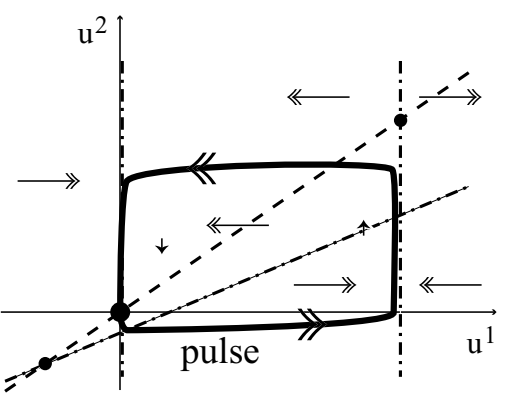

(b)

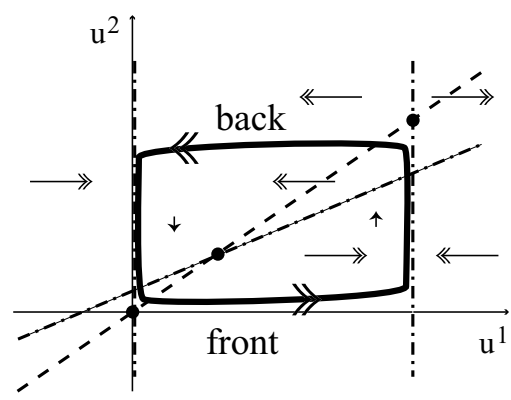

Fig. 2.16. Excitable (a) and oscillatory (b) media; homoclinic pulses and periodic travelling waves for $\varepsilon \searrow 0$. Isoclines in absence of diffusion. See also (2.114) for the excitable case (a).

The $u^{2}$-values are nearly constant through each $u^{1}$-jump, in the singular limit $\varepsilon \searrow 0$. The diffusive $u^{2}$-equation, however, constitutes a highly nonlocal and nonlinear coupling of the appropriate local wave speeds $c_{0}=c_{0}\left(u^{2}\right)$ in the curvature motion (2.100) or (2.112). It seems tempting to derive an equation for the tip motion, in this case, involving local information on the "pulse" curve at the tip itself. See [MiZy91] for a phenomenological attempt.

Spatially periodic stable travelling waves can likewise be realized, for appropriate $a, b$ and small $\varepsilon$, if we modify the equation $u_{t}^{2}$ by subtracting a suitable positive constant; see also Figure 2.16(b). This corresponds to an oscillatory medium. 
In the following section we will take an entirely different approach, based on reaction-diffusion systems in the plane $x \in \Omega=\mathbb{R}^{2}$, but neither restricted to, nor complicated by, singular perturbations. In particular, in Section 2.4.3, we address the issue of primary bifurcation of spiral waves from spatially homogeneous equilibria in the presence of continuous spectrum. In Chapter 2.5, we will further pursue this approach to include secondary bifurcations from Archimedean rotating waves.

\subsubsection{Archimedean Spiral Waves in Radial Dynamics}

In Section 2.4.2, we collect some basic characteristics of spiral waves in reaction-diffusion systems: rigid rotation and Archimedean shape. Section 2.4.2 clarifies, in which sense spiral waves "emit" wave trains. We summarize some principal features of a spiral wave, in Section 2.4.2.

Rigid rotation and asymptotic wavetrains Proving existence of spiral waves in reaction-diffusion systems has been a long standing mathematical problem. In spite of their prevalence and robustness in experiments as well as in numerical simulations, proofs of existence for spiral waves are rare. Given the empirical evidence for existence, one might suspect that the existence problem is of purely mathematical interest. We believe, however, that a proper understanding of the existence problem also leads to a systematic approach to many observable stability and instability phenomena involving spiral wave dynamics.

Consider a reaction-diffusion system in $x \in \mathbb{R}^{2}$, the unbounded plane,

$$
u_{t}=D \Delta_{x} u+f(u), \quad x \in \mathbb{R}^{2} .
$$

We aim at a precise definition of what we understand by an Archimedean spiral wave solution to (2.115).

First of all, the simplest spiral waves observed in chemical experiments are rigidly rotating. Rigid rotation is easily described in polar coordinates $(r, \varphi)$, as we saw in Section 2.4.1. The central point of our discussion of Archimedean spirals in Chapters 2.4 and 2.5, on top of that mere convenience, will be a particular view on the radial coordinate $r$ : we will view radius $r$, rather than time $t$, as a new, spatial "time" variable - much in the same way as we have viewed the spatial variable $x$ as "time" in our discussion of travelling waves in Chapter 2.3. We refer to this view point, which was first used in [Sc98] for the spiral wave problem and further exploited in [Sc01], as radial dynamics.

In polar coordinates $(r, \varphi)$, rigid rotation allows us to write the spiral wave solution $q_{*}$ in the form

$$
u(t, r, \varphi)=q_{*}\left(r, \varphi+\omega_{*} t\right) .
$$

Here, $\omega_{0}$ is the temporal frequency of rotation of the spiral. Inserting this Ansatz into the reaction-diffusion system, we find 


$$
D\left(u_{r r}+\frac{1}{r} u_{r}+\frac{1}{r^{2}} u_{\psi \psi}\right)-\omega_{*} u_{\psi}+f(u)=0,
$$

a partial differential equation of elliptic type for $u=q_{*}(r, \psi)$, with $\psi=$ $\varphi+\omega_{*} t$. Note, however, that the operator $\omega_{*} \partial_{\psi}$ is not relatively bounded with respect to the Laplacian. Indeed, the derivative $\partial_{\psi}$ cannot be bounded by $r^{-2} \partial_{\psi \psi}$. As a consequence, the smooth function $\sin \left(x_{1}\right)$, for example, belongs to the domain of the Laplacian on spaces of bounded, uniformly continuous functions, but the angular derivative $x_{2} \partial_{x_{1}} \sin \left(x_{1}\right)$ is unbounded.

An additional characteristic of many observed spiral waves is their Archi" medean shape. We require the profile to be Archimedean far away from the center of rotation,

$$
\left|q_{*}(r, \psi)-q_{\infty}\left(r-\left(\psi / k_{\infty}\right)\right)\right| \longrightarrow 0 \text { for } r \rightarrow \infty,
$$

uniformly in $\psi$, for some function $q_{\infty}(\xi)$. Note that $q_{\infty}$ is constant along Archimedean spirals $\psi=k_{\infty} r+\psi_{0}$. Convergence (2.118) is the "boundary condition" that we will impose at $r=\infty$ for our spiral wave solution to the elliptic system (2.117). The asymptotic profile of the spiral wave, $q_{\infty}$, has to satisfy certain compatibility conditions. First, $q_{\infty}$ is $2 \pi / k_{\infty}$-periodic in its argument by continuity. Second, if we formally let $r \rightarrow \infty$ in $(2.117)$, we find the condition

$$
D q_{\infty}^{\prime \prime}+\frac{\omega_{*}}{k_{\infty}} q_{\infty}^{\prime}+f\left(q_{\infty}\right)=0 .
$$

This is nothing else but the travelling-wave equation for one-dimensional periodic wavetrain solutions $q\left(x-\left(\omega_{*} / k_{\mathrm{wt}}\right) t\right)$ of

$$
u_{t}=D u_{x x}+f(u)
$$

with speed $\omega_{*} / k_{\infty}$ and spatial period $2 \pi / k_{\infty}$. We therefore call $q_{\infty}$ the asymptotic wavetrain of the spiral wave solution. The constant $k_{\infty}$ appearing in (2.118) is the wavenumber of the asymptotic wavetrain. The speed of the asymptotic wavetrain is computed from the standard relation

$$
c_{\infty}=\omega_{*} / k_{\infty}
$$

For fixed, arbitrary, speed $c$, periodic solutions to the travelling-wave equation (2.119) are typically isolated. The spatial period $2 \pi / k$ depends nontrivially on $c$. Using (2.120) with $k_{\infty}=k, c_{\infty}=c$, we can turn this dependence in a relation between $\omega$ and $k$. We call this relation the nonlinear dispersion relation of the asymptotic wavetrain

$$
d_{\mathrm{nl}}(\omega, k)=0 .
$$

The spiral "selects" a particular solution $\omega=\omega_{*}, k=k_{\infty}$ of (2.121). 
Linear and nonlinear group velocities Most spiral waves that have been observed in chemical experiments share an additional property: wavetrains are "emitted" by the center. Spirals with this property are sometimes referred to as active spirals. Mathematically, the emission of wavetrains is characterized by a positive group velocity $c_{\mathrm{g}}>0$ of the asymptotic wavetrains in the radial direction. The concept of group velocity we are referring to, here, is a slight generalization of the definition in equation (2.69). There are actually two equivalent definitions of the group velocity for wavetrains. The simplest definition would refer to the nonlinear dispersion relation (2.121). Assuming that we can solve for $\omega_{\infty}$ as a function of $k_{\infty}$, we define the nonlinear group velocity through

$$
c_{\mathrm{g}, \mathrm{nl}}=\frac{\mathrm{d} \omega_{\infty}}{\mathrm{d} k_{\infty}},
$$

evaluated at the wavenumber of the wavetrain, $k_{\infty}$.

We say that a spiral waves emits the asymptotic wavetrain, if the nonlinear group velocity of the asymptotic wavetrain is positive.

Another definition of the group velocity would refer to the linearization about the wavetrain $q_{\infty}$ in a comoving frame of speed $\omega_{*} / k_{\infty}$,

$$
w_{t}=D w_{\xi \xi}+\frac{\omega_{*}}{k_{\infty}} w_{\xi}+f^{\prime}\left(q_{\infty}(\xi)\right) w .
$$

At the end of Section 2.3.1, (2.80)-(2.83), we explained how to solve this linear parabolic equation with spatially periodic coefficients by means of a generalized Fourier transformation. Indeed, the general solution can be decomposed into Bloch waves $w(t, \xi ; k)$,

$$
w(t, \xi ; k)=\mathrm{e}^{\lambda(\mathrm{i} k) t-\mathrm{i} k \xi} w_{0}(\xi ; k),
$$

where $w_{0}$ solves

$$
\left(\lambda(\mathrm{i} k)+c_{\infty} \mathrm{i} k\right) w_{0}=D\left(\frac{\mathrm{d}}{\mathrm{d} \xi}-\mathrm{i} k\right)^{2} w_{0}+c_{\infty} \frac{\mathrm{d}}{\mathrm{d} \xi} w_{0}+f^{\prime}\left(q_{\infty}(\xi)\right) w_{0} .
$$

Here, $w_{0}(\xi ; k)=w_{0}\left(\xi+\left(2 \pi / k_{\infty}\right) ; k\right)$ is spatially periodic with the period of the wavetrain $q_{\infty}$ and the Bloch wavenumber $k$ introduces a second spatial period. The eigenvalue $\lambda$ and the Bloch wavenumber $k$ are related by a dispersion relation $d_{\operatorname{lin}}(\lambda, \mathrm{i} k)=0$, see (2.83). Assuming that we can solve this equation for $\lambda=\lambda(k)$, with $\lambda(0)=0$, the linear group velocity is computed in analogy to $(2.69)$

$$
c_{\mathrm{g}, \operatorname{lin}}=c_{\infty}+\left.\operatorname{Im} \frac{\mathrm{d} \lambda}{\mathrm{d} k}\right|_{k=0} .
$$

The summand $c_{\infty}$ accounts for the fact that we compute the dispersion relation in a comoving frame, whereas we are interested in the group velocity in the steady frame. Note that we can define a linear group velocity for different values of $k$, and actually on different solution branches $\lambda^{\ell}(\mathrm{i} k)$ of the dispersion relation $d_{\operatorname{lin}}$, again in analogy to $(2.69)$. 
Lemma 2.4.1 Linear and nonlinear group velocities of periodic wavetrains, as defined in (2.122) and (2.126), coincide. In particular, whenever one of the two is well-defined, both are well-defined and

$$
c_{\mathrm{g}, \mathrm{nl}}=c_{\mathrm{g}, \mathrm{lin}}=: c_{\mathrm{g}} .
$$

The proof of the lemma reduces the computation of both group velocities to a boundary-value problem, which can then readily be solved by LyapunovSchmidt reduction.

Characterizing Archimedean spirals In Sections 2.4.2 and 2.4.2 we have collected three properties of certain chemical spiral waves: rigid rotation, asymptotic Archimedean shape, and positive group velocity of the asymptotic wavetrains. Heading for an open class of "robust" solutions that resemble Archimedean spirals, we incorporate the apparent robustness of spiral waves with respect to changes in parameter values into our characterization. So far, we have set up a boundary-value problem for spiral wave solutions. Preferably, robustness proofs for spiral would rely on an implicit function theorem in a suitable function space. Unfortunately the properties of spiral waves, as collected above, prevent us from an application of the implicit function theorem, since the linearization of (2.117) lacks the Fredholm property.

More specifically, consider an Archimedean spiral wave solution $q_{*}(r, \psi)$ to $(2.117)$ on $x \in \mathbb{R}^{2}$. Linearizing (2.115) at $q_{*}$ in a corotating frame yields

$$
w_{t}=D \Delta_{r, \psi} w-\omega_{*} \partial_{\psi} w+f^{\prime}\left(q_{*}(r, \psi)\right) w=: \mathcal{L}_{*} w .
$$

The elliptic operator $\mathcal{L}_{*}$ is not Fredholm on $L^{p}$-spaces or spaces of continuous functions; see [Sa\&al99, Lemma 6.4], for example. We therefore cannot conclude persistence of a spiral wave solution under slight variation of diffusion coefficients or reaction kinetics, simply by an implicit function theorem.

In the remainder of this section, we aim at a definition of a spiral wave that will allow us to conclude robustness. The main idea is to invoke Lemma 2.3.3, relating Fredholm properties in exponentially weighted spaces to group velocities. Analogously to the weighted $L^{2}$-spaces in (2.73) on the real line $x \in \mathbb{R}$, we introduce the exponentially weighted spaces

$$
L_{\eta}^{2}\left(\mathbb{R}^{2}\right):=\left\{u \in L_{\mathrm{loc}}^{2}\left(\mathbb{R}^{2}\right) ;\|u\|_{L_{\eta}^{2}}^{2}:=\int_{\mathbb{R}^{2}}\left|\mathrm{e}^{\eta|x|} u(x)\right|^{2}<\infty\right\},
$$

for weights $\eta \in \mathbb{R}$ in the radial direction $r=|x|$.

Definition 2.4.2 An Archimedean spiral is a bounded rotating-wave solution $q_{*}\left(r, \varphi-\omega_{*} t\right)$ of the reaction-diffusion equation (2.115), with some nonzero rotation frequency $\omega_{*}$, which converges to plane wavetrains in the farfield

$$
\left|q_{*}(r, \psi)-q_{\infty}\left(r-\left(\psi / k_{\infty}\right)\right)\right| \longrightarrow 0, \quad \text { for } r \rightarrow \infty,
$$

uniformly in $\psi \in[0,2 \pi]$. We call an Archimedean spiral transverse, if the following conditions hold: 
(i) there exists $\eta_{0}<0$ such that the linearization $\mathcal{L}_{*}$, defined in (2.127), is Fredholm with index 0 in $L_{\eta}^{2}$, for all $\eta_{0}<\eta<0$, and the dimension of the generalized kernel is one;

(ii) there is $\eta_{1}>0$ such that the linearization $\mathcal{L}_{*}$ is Fredholm with index -1 for all $\eta$ with $0<\eta<\eta_{1}$.

The definition encodes positive group velocity of the wavetrain in the negative Fredholm index of the linearization; see Proposition 2.3.1, Lemma 2.3.3, and the discussion of the linearization in Section 2.5.3, below. It also reflects simplicity of the trivial zero eigenvalue $\lambda=0$. For fronts and pulses, we have taken advantage of this property in order to conclude transverse crossing of stable and unstable manifolds in the travelling-wave equation, see Section 2.3.2. It is for this geometric interpretation that we refer to Archimedean spirals satisfying Definition 2.4.2(i),(ii) as transverse Archimedean spirals.

Theorem 2.4.3 [SaSc00d] Assume the reaction-diffusion system (2.115) with analytic reaction kinetics $f=f(u)$ possesses a transverse Archimedean spiral wave solution. Then, for any reaction kinetics $\tilde{f}, C^{1}$-close to $f$, and any diffusion matrix $\tilde{D}$ close to $D$, the perturbed reaction-diffusion system

$$
u_{t}=\tilde{D} \Delta u+\tilde{f}(u)
$$

also possesses a transverse Archimedean spiral wave.

For the proof of the theorem, we rewrite (2.117) in radial dynamics using $r=|x|$ as "time"; see [Sc98]. We then interpret a spiral wave as a heteroclinic front solution connecting the center $r=0$ with the wavetrains in the farfield $r=\infty$. The radial dynamics formulation of (2.117) reads

$$
\begin{aligned}
& U_{r}=V \\
& V_{r}=-\frac{1}{r} V-\frac{1}{r^{2}} \partial_{\psi \psi} U-D^{-1}\left(-\omega_{*} \partial_{\psi} U+f(U)\right) .
\end{aligned}
$$

Note how spiral waves $q_{*}(r, \psi)$ actually turn into heteroclinic orbits $(U, V)(r, \cdot)$, just like fronts in one space-dimension, Section 2.3.1, or the modulated fronts in Section 2.3.2. We view equation (2.129) as a non-autonomous differential equation on a Hilbert space $(U, V)(r, \cdot) \in H^{1}\left(S^{1}\right) \times L^{2}\left(S^{1}\right)$. Just like the travelling-wave ODE for modulated fronts (2.89), the initial value problem for the elliptic system (2.129) is ill-posed. Stable and unstable manifolds exist, but turn out to be infinite-dimensional. An additional complication arises since the term involving the highest derivative $\frac{1}{r^{2}} \partial_{\psi \psi} U$ is non-autonomous, singular at the origin $r=0$, and degenerate in the farfield $r=\infty$.

The limit $r \rightarrow 0$ of the core region is easily understood in the scaling $r=\mathrm{e}^{\tau}$, where we find

$$
\begin{aligned}
U_{\tau} & =W \\
W_{\tau} & =\partial_{\psi \psi} U-\mathrm{e}^{2 \tau} D^{-1}\left(-\omega_{*} \partial_{\psi} U+f(U)\right) .
\end{aligned}
$$


In the farfield limit $r \rightarrow \infty$, we recover the equation for modulated travelling waves (2.89),

$$
\begin{aligned}
& U_{r}=V \\
& V_{r}=-D^{-1}\left(-\omega_{*} \partial_{\psi} U+f(U)\right) .
\end{aligned}
$$

Spiral waves are solutions which converge to constant functions $U=U_{0}, V=$ 0 in the center of rotation $r=0$. For $r \rightarrow \infty$, they approach the asymptotic wavetrain $U=q_{\infty}\left(r-\left(\psi / k_{\infty}\right)\right), V=u_{\infty}^{\prime}\left(r-\left(\psi / k_{\infty}\right)\right)$. In this sense, spiral waves are heteroclinic orbits in the non-autonomous differential equation (2.129), connecting an equilibrium at $\tau=\log r=-\infty$ to a periodic orbit at $r=+\infty$.

Transversality of Archimedean spirals, in the sense of Definition 2.4.2, implies that the associated heteroclinic orbit in spatial dynamics consists of a transverse crossing of the stable manifold of the asymptotic periodic orbit, at $r=\infty$, and the unstable manifold at $r=0$. Transverse crossing, here, again refers to a transverse intersection of stable and unstable manifolds in the extended phase space, augmented by the spiral frequency parameter $\omega_{*}$; see also Section 2.3.2. We return to this geometric picture for the linearized equation in more detail in Section 2.5.3, below.

The proof of Theorem 2.4.3 is therefore based on smoothness of centerstable and center-unstable manifolds in the radial dynamics formulation, and a robustness argument for transverse intersections. Analyticity of $f$ is used for uniqueness of solutions to the ill-posed initial-value problem in the radial dynamics formulation.

\subsubsection{Bifurcation to Spiral Waves}

Theorem 2.4.3 shows that transverse Archimedean spiral waves are robust. We now show that transverse Archimedean spiral waves actually exist. We construct these spirals through bifurcation from a spatially homogeneous equilibrium in the unbounded plane $x \in \mathbb{R}^{2}$, in the presence of critical continuous spectrum.

We consider reaction-diffusion equations with the pure reaction-kinetics undergoing a Hopf bifurcation. We assume that $f(u ; \mu)$ depends on a parameter $\mu$ and $f(0 ; \mu)=0$ for $\mu$ close to zero. The linearization $\partial_{u} f(0 ; 0)$ is assumed to possess a pair of purely imaginary eigenvalues $\pm \mathrm{i} \omega_{\mathrm{H}}$, with eigenvectors $u_{*}, \bar{u}_{*} \in \mathbb{C}^{N}$. We assume that all other eigenvalues are contained in the left complex half plane. Performing a center-manifold reduction and a subsequent normal-form transformation, the Hopf bifurcation in the pure kinetics $u_{t}=f(u ; \mu)$ can be reduced to a two-dimensional ordinary differential equation of the form

$$
\dot{z}=\lambda(\mu) z+\beta z|z|^{2}+\mathrm{O}(|z|)^{5}
$$


with eigenvalue $\lambda(0)=\mathrm{i} \omega_{\mathrm{H}}$ and complex cubic normal form coefficient $\beta \in$ $\mathbb{C}$. We assume that $\lambda^{\prime}(0)>0$, crossing of eigenvalues, and $\operatorname{Re} \beta<0$. The linearization of the spatially dependent reaction-diffusion system in $u=0$,

$$
u_{t}=D \Delta u+\partial_{u} f(0 ; 0) u,
$$

can be analyzed after Fourier transform from the dispersion relation

$$
d(\lambda, \mathrm{i} k)=\operatorname{det}\left(-D k^{2}+\partial_{u} f(0 ; 0)-\lambda\right)=0, \quad k \in \mathbb{R} .
$$

For wavenumbers $k \in \mathbb{R}$ near zero, the eigenvalue $\lambda=\mathrm{i} \omega_{\mathrm{H}}$ continues to a critical spectral curve $\lambda(\mathrm{i} k ; \mu)$, with $\lambda(0 ; 0)=\mathrm{i} \omega_{\mathrm{H}}$ and expansion

$$
\lambda(\mathrm{i} k ; 0)=\mathrm{i} \omega_{\mathrm{H}}+\alpha k^{2}+\mathrm{O}\left(k^{4}\right) .
$$

Theorem 2.4.4 [Sc98] There exists a nonempty open set $\mathcal{U} \subset C^{3}\left(\mathbb{R}^{N}\right)$ of nonlinearities $f=f(u)$, such that for all $f \in \mathcal{U}$ the reaction-diffusion system (2.115) possesses a transverse Archimedean spiral wave, in the sense of Definition 2.4.2.

More specifically, assume that the reaction-diffusion system undergoes a Hopf bifurcation such that

(i) $\lambda(0 ; 0)=\mathrm{i} \omega$ is a simple zero of (2.133) and the only purely imaginary solution to (2.133), for any $k \in \mathbb{R}$;

(ii) $\partial_{\mu} \operatorname{Re} \lambda(0 ; 0)>0$;

(iii) for $\alpha$ and $\beta$, as defined in (2.132) and (2.134), we have $\operatorname{Re} \beta<0, \operatorname{Re} \beta<$ 0 and $|\arg (\beta / \alpha)|<\delta$ sufficiently small.

Then, for sufficiently small $\mu>0$, there exists a transverse Archimedean spiral wave solution of (2.115).

One approach to Theorem 2.4.4 would be to approximate the reactiondiffusion system by a complex Ginzburg-Lan"-dau equation

$$
A_{\tilde{t}}=\alpha \Delta_{\tilde{x}} A+A+\beta A|A|^{2}, \quad \tilde{x} \in \mathbb{R}^{2}, A, \alpha, \beta \in \mathbb{C},
$$

and then look for rigidly rotating spiral wave solutions. For persistence of spiral waves however, we might still have to rely on a spatial dynamics formulation as in Theorem 2.4.3.

The proof given in [Sc98] splits into three parts. The first part contains a reduction of the radial dynamics (2.89) to a finite-dimensional center manifold, which contains all solutions of (2.129) close to the equilibrium $(U, V) \equiv 0[\mathrm{Sc} 98$, Theorem 1, Proposition 5]. On the center manifold, we find the bifurcation equation

$$
A_{r r}=-\frac{1}{r} A_{r}-\frac{1}{r^{2}} A+A-\mathrm{i} \tilde{\omega} A+(\beta / \alpha) A|A|^{2},
$$

for $A(r) \in \mathbb{C}$, to leading order in an appropriate scaling. The parameter $\tilde{\omega}$ depends on the frequency of rotation $\omega_{*}$. In the second part, this reduced 
equation is shown to possess a transverse heteroclinic orbit, asymptotic to plane wave solutions with positive group velocity [Ha82, KoHo81, Sc98]. The proof is concluded with a persistence result under higher-order perturbations, similar to Theorem 2.4.3.

The results in [Sc97, Sc98] also show that spatially localized rotating wave solutions $u(r, \varphi-\omega t)$ with $u(r, \cdot) \rightarrow 0$ for $r \rightarrow \infty$ bifurcate if the Hopf bifurcation is subcritical. To our knowledge, these patterns have not been observed in experiments, yet.

\subsection{Two Space Dimensions: Bifurcations from Spiral Waves}

In this chapter we discuss several instability mechanisms for the spiral rotating wave solutions which are constructed in the previous chapter. We start with a biased review of experimentally observed instabilities, in Section 2.5.1. We then consider instabilities of Hopf type, caused by point spectrum. Such instabilities lead to meandering motions of the spiral tip and to periodic shape fluctuations. Equivariant bifurcations under the noncompact symmetry group $S E(m)$ are an appropriate framework here, as we will discuss in Section 2.5.2.

Beyond the two-frequency meandering motion, more complicated motions of the spatial tip have been observed [Un\&al93]. Attempts to explain these motions strongly rely on a finite-dimensional description of the instability, coupled to the motion on the non-compact group $S E(2)$ [FiTu98, Go\&al97, As\&al01]. The motion of the tip, often referred to as hypermeander (although already meander originally refers to an irregular winding of river beds) can under certain assumptions be shown to trace Brownian motion paths; see Section 5.2.7.

In Section 2.5.3, we analyze the linearization about Archimedean spiral waves in a more systematic way. In particular, we classify continuous spectra and the super-spiral shapes of eigenfunctions. The results in this section are then compared with experiments and numerical simulations in Section 2.5.4: the spatio-temporal patterns arising in spiral breakup and meander instabilities are related to spectral properties of the primary Archimedean spiral.

The discussion of continuous spectra in Sections 2.5.3 and 2.5.4 is largely independent of Section 2.5.2 and closely follows the exposition on radial dynamics in Sections 2.4.2, 2.4.3 and on the one-dimensional case, Chapter 2.3.

\subsubsection{Phenomenology of Spiral Instabilities}

Beyond rigidly rotating spiral waves, destabilization of individual spiral waves and subsequent transition to still richer spatio-temporal patterns has been observed in chemical experiments. In this section, we review some experimentally and numerically observed instabilities. 
Meandering of the spiral tip was observed in [Ja\&al89, SkSw91, Ne\&al, BrEn93, Un\&al93], among others. Temporally, the spiral motion becomes a two-frequency motion. For example, in a two-species reaction-diffusion system $N=2$, we may trace the evolution of a point $x=z_{*}(t)$ in physical space $x \in \mathbb{R}^{2}$, where the concentration vector possesses a fixed, prescribed value $u_{*} \in \mathbb{R}^{2}$. The curve $z_{*}(t)$ describes a one-frequency motion on a circle before the transition to meander movement. After transition the curve $x_{0}=x_{0}(t)$ becomes an epicycle, as shown in Figure 2.17, 2.22. We refer to this (nonunique) point as the tip of the spiral; see Section 5.2.4. Beyond the two-frequency meandering motion. We recall more complicated motions of the spiral tip have been observed; see Section 5.2.7.

Explanations based on a curvature description of the wavetrain dynamics in the spirit of Section 2.4.1, together with an additional equation for the core of the spiral, were proposed in [MiZy91]. Barkley [Ba92] was the first to suggest simple Hopf bifurcation in the presence of Euclidean symmetry as the mechanism which leads to meandering. Barkley later discovered numerically that drift of the tip is related to a resonance between Hopf frequency and rotation frequency of the spiral wave [Ba93]. The numerical and theoretical predictions have been confirmed in careful experiments. In particular, the Hopf-typical square-root scaling of the amplitude of the instability as a function of the parameter driving the instability has been verified [Ou\&al].

The patterns in the farfield appear as prominent super-imposed spiral-like regions, where the local wavelength is larger than the average. The numerical computation of critical eigenfunctions reveals an apparent dramatic change of the radial growth behavior of the eigenfunction when the bifurcation parameter is varied close to criticality: before instability, the amplitude of the eigenfunction appears to grow with the distance from the center of rotation, whereas after instability, the eigenfunction seems to be localized. Experimentally, the instability appears to be localized at the center of rotation in physical space. However, radial decay is very weak; see [Ou\&al].

Spiral breakup is a phenomenologically different instability where, as in the meander instability prominent super-patterns occur in the farfield $|x| \rightarrow \infty$. This time, however, perturbations grow in amplitude with distance from the center; see [BäEi93, BäOr99, Be\&al97, ZhOu00]. Before spiral breakup, the perturbations of the spiral wave again take the form of superimposed spirals of compression and expansion of the local wavelength of the wavetrains. Instability finally leads to visible collision of emitted pulses. Collision is generally referred to as breakup and eventually leads to the formation of new spiral cores, far away from the center of the primary spiral.The transition to instability is not sharp and depends on the size of the domain. We refer to this instability mechanism as farfield breakup.

Spiral breakup, i.e. collision of wavetrains, can also occur close to the core region, $r \rightarrow 0$, of the primary spiral. After collision, new spirals form close to the core of the primary spiral, which break up immediately. This mechanism 
of instability leads to very incoherent spatio-temporal states, with many small spiral domains; see [Ar\&al94, BäOr99]. We refer to this scenario of instability as core breakup.

Both spiral breakup scenarios, farfield and core breakup, may occur as instabilities of rigidly rotating or meandering spirals [ZhOu00, BäOr99].

Aranson and Kramer [Ar\&a192], considering the complex Ginzburg-Lan"dau model equation, point out that the lack of a sharp transition to instability in spiral breakup is caused by transport of the wavetrains, rendering the instability convective: perturbations increase in amplitude, but decay at each fixed point in physical space. Sensitivity to instability can be increased by either further increasing the bifurcation parameter, or, by increasing the size of the domain. Baer et al. compare breakup instabilities with instabilities of one-dimensional wavetrains emitted by a Dirichlet source [BäOr99]. Striking qualitative and quantitative similarity suggests that spiral breakup is caused by an instability of the wavetrains in the farfield.

We briefly mention three other instability phenomena without attempting a complete explanation, here. The retracting wave bifurcation [MiZy91] manifests itself in a decrease of the spiral frequency $\omega_{*}$ to zero. The radius of the circle $x_{0}(t)$ that a fixed point $u_{0}$ on the spiral profile describes diverges until the spiral finally leaves the window of observation. The instability is partially explained in [As\&al99] invoking a finite-dimensional reduction procedure. Two-dimensional, fingering-type instabilities of wavetrains, emitted by a spiral wave, have been studied in [MaPa97]. Period-doubling of a homogeneous oscillation which induced complicated bifurcations of the emitted wavetrains, has been observed in oscillatory media [Go\&al98].

We do not attempt to list particular models giving rise to the plethora of phenomena around spiral waves. Most models center around two- or threespecies reaction-diffusion equations with excitable or oscillatory kinetics. A different line of investigations concentrates on the complex Ginzburg-Lan"dau equation, where in particular the wavetrain dynamics are amenable to explicit, algebraic computations.

Our emphasis is on the general framework, assuming existence of spiral waves without recurring to the precise kinetics. Instability scenarios will be explained from certain spectral assumptions on the linearization, rather than discussions of oscillatory versus excitable kinetics.

As a general guideline, we expect instabilities to be caused either by essential spectrum or by point spectrum crossing the imaginary axis. Essential spectrum crossing the axis will typically not lead to an instability in bounded domains, where the absolute spectrum, strictly to the left of the essential spectrum, decides upon stability.

\subsubsection{Meandering Spirals and Euclidean Symmetry}

In this section we consider a given rotating wave solution $u=q_{*}\left(r, \varphi-\omega_{*} t\right)$ of a reaction-diffusion system 


$$
u_{t}=D \Delta u+f(\mu, u) .
$$

We consider $u \in \mathbb{R}^{N}$, a positive diagonal diffusion matrix $D, x \in \mathbb{R}^{m}$, mostly $m=2$, and a scalar bifurcation parameter $\mu$. For $f \in C^{2}$, system (2.137) fits into the abstract framework of analytic semigroup theory on a Banach space $X,[\mathrm{He} 81, \mathrm{~Pa} 83]$, but we prefer to formulate results in the more specific setting (2.137). Specifically we may think of $X=L^{2}$, excluding the Archimedean spirals of Chapter 2.4, which do not decay in amplitude for $|x| \rightarrow \infty$. Suitable subspaces $X$ of $B U C$, the space of bounded uniformly continuous functions, are another possible choice. Although $B U C$ does accommodate Archimedean spirals, these may exhibit critical continuous spectrum — a complication which we postpone to Section 2.5.3.

Euclidean equivariance Since the Laplacian $\Delta$ as well as the point evaluation nonlinearity commute with translations $S \in \mathbb{R}^{m}$ and rotations $R \in S O(m)$ of spatial profiles $u \in X$, we observe equivariance of the semiflow generated by (2.137) under the Euclidean symmetry group

$$
G=S E(m)=\left\{(R, S) ;\left(R \in S O(m), S \in \mathbb{R}^{m}\right\} .\right.
$$

The action on $g=(R, S) \in G$ on $u=u(x)$ is given explicitly by

$$
((R, S) u)(x):=u\left((R, S)^{-1} x\right) .
$$

Note that the action on $x \in \mathbb{R}^{m}$ is affine,

$$
(R, S) x:=R x+S,
$$

whereas the action on $u \in X$ is linear. Equivariance of (2.137) under $G$ means that $u(t) \in X$ is a solution if, and only if, $g u(t) \in X$ is a solution, for every fixed $g \in G$. For a general background of bifurcation theory in the presence of (mostly compact) symmetry groups we refer to [ChLa00, GoSc85, Go\&al88, Va82].

The group $G=S E(m)$ can be viewed as a Lie subgroup of the special linear group $S L(m+1)$ of real $(m+1) \times(m+1)$ matrices with determinant one, via the embedding

$$
\begin{aligned}
S E(m) & \rightarrow S L(m+1) \\
(R, S) & \mapsto\left(\begin{array}{cc}
R & S \\
0 & 1
\end{array}\right)
\end{aligned}
$$

For example, $(R, S)^{-1}=\left(R^{-1},-R^{-1} S\right)$.

The embedding (2.141) is also useful for practical computations of the Lie algebra $s e(m)$ and of the exponential map exp $: s e(m) \rightarrow S E(m)$. For example $\mathbf{a}:=(\mathbf{r}, \mathbf{s}) \in \operatorname{se}(m)$ is given by a skew symmetric matrix $\mathbf{r} \in \operatorname{so}(m)$ and by $\mathbf{s} \in \mathbb{R}^{m}$ with explicit exponential

$$
\exp (\mathbf{r}, \mathbf{s})=\left(\exp \mathbf{r}, \mathbf{r}^{-1}(\exp (\mathbf{r})-\mathrm{id}) \mathbf{s}\right)
$$


For nonzero $\mathbf{r} \in s o(2)$, we therefore see how any exponential $\exp (\mathbf{a} t)$ is conjugate to a pure rotation $\mathbf{s}=0$, by a suitable translation. Identifying $\mathbf{r} \in s o(2)$ with the purely imaginary number $\mathrm{i} \omega$, we can also write rotations $R \in S O(2)$ as $R=\exp (\mathbf{r} t)=\exp (\mathrm{i} \omega t)$. See [Fi\&al96, Ch. 4] for many more examples.

In this abstract setting, a rotating wave solution $u(t)=u(t, \cdot) \in X$ of (2.137) takes the form

$$
u(t)=\exp \left(\mathbf{a}_{0} t\right) q_{*},
$$

for some $q_{*} \in X$. The polar coordinate formulation (2.116) of Section 2.4.2 coincides with (2.143) for $\mathbf{a}_{0}=\left(\mathbf{r}_{0}, 0\right)$ and $\mathbf{r}_{0}=\mathrm{i} \omega_{*}$. More generally, solutions of the form (2.143), with an arbitrary element $\mathbf{a}_{0}$ of the Lie algebra $\operatorname{alg}(G)$ of $G$, are called relative equilibria: the time orbit (2.143) is entirely contained in the group orbit $G q_{*}$ and hence does not exhibit any shape change. Travelling waves, where $G=(\mathbb{R},+)$, are another simple example; see Chapter 2.3.

Relative center manifolds We now describe the center-manifold reduction near relative equilibria (2.143) due to [Sa\&al97a, Sa\&al97b, Sa\&al99]. We restrict to the slightly simpler case of symmetry groups $G \leq S E(m)$. Fix the parameter $\mu=\mu_{0}$. Assume the reaction kinetics $f=f(u)$ are smooth, $C^{\infty}$, and fix $k \in \mathbb{N}$ arbitrary. We first pass to a "rotating" or "comoving" coordinate frame $\tilde{u}:=\exp \left(-\mathbf{a}_{0} t\right) u$, where $u(t)$ becomes an equilibrium $\tilde{u}=: q_{*}$. By $G$-equivariance, this transformation leaves (2.137) autonomous. Assume that the group action is continuous on the relative equilibrium $q_{*}$. The central assumption to Theorem 2.5.1, below, is that the linearization at the equilibria $G q_{*}$ possess only point spectrum in $\{\operatorname{Re} \lambda \geq-\delta\} \subset \mathbb{C}$, for some $\delta>0$. Let $W$ denote the corresponding finite-dimensional generalized eigenspace.

Theorem 2.5.1 [Sa\&al97a, SaÉal97b, Sa\&́al99] Under the above assumptions there exists a $G$-invariant neighborhood $\mathcal{U}$ of the group orbit $G q_{*}$ of the relative equilibrium $q_{*}$ and a $G$-invariant $C^{k}$ center manifold $\mathcal{M} \subseteq \mathcal{U}$ with differentiable proper $G$-action on $\mathcal{M}$. The $C^{k}$-manifold $\mathcal{M}$ is time-invariant within $\mathcal{U}$, and tangent to the center eigenspace $W$ at $q_{*} \in \mathcal{M}$. Moreover $\mathcal{M}$ contains any solution $u(t), t \in \mathbb{R}$, which remains in $\mathcal{U}$ for all positive and negative times $t$.

Since the center manifold $\mathcal{M}$ is based on a parameterization over the group orbit $G q_{*}$ of the relative equilibrium $q_{*}$, we call $\mathcal{M}$ a relative center manifold.

Properness of the $G$-action on $\mathcal{M}$ is a technical property, which asserts that the map

$$
\begin{aligned}
G \times \mathcal{M} & \rightarrow \mathcal{M} \times \mathcal{M} \\
(g, u) & \mapsto(u, g u)
\end{aligned}
$$

is proper: images of closed subsets are closed and preimages of points are compact. For example, consider the isotropy $H$ of $q_{*} \in \mathcal{M}$ : 


$$
H=G_{q_{*}}:=\left\{h \in G ; h q_{*}=q_{*}\right\} .
$$

The isotropy $H$ consists of the symmetries of the solution $q_{*}$ in the group $G$. Note that isotropies are compact, for proper group actions. Indeed $H \times\{u\}$ is the preimage of $\left(q_{*}, q_{*}\right)$ under the proper map (2.144), and hence compact.

We caution the reader that the eigenspace $W$ is not $G$-invariant, in general. For example $G=S E(2)$ does not possess any nontrivial finitedimensional $G$-invariant subspace in $L^{2}$. In fact, $g \in G$ will move $W$ to contain the tangent space $T_{g q_{*}} G q_{*}$ which may only intersect trivially with $W$. In particular, the center manifold $\mathcal{M}$ cannot be written as a graph of a globally $G$-equivariant map over the tangent space $W$. This situation differs markedly from the case of compact symmetries $G$, where $X$ decomposes into finite-dimensional $G$-invariant subspaces.

The proof of theorem 2.5.1 first exploits finite-dimensionality of the center-eigenspace in order to prove smoothness of the group action on spectral projections associated with $W$ and on individual elements of $W$. This allows for a smooth parameterization of the group orbit $G\left(q_{*}+W\right)$. The main difficulty is that the group action on the normal bundle, consisting of the strongly stable subspace, is not even strongly continuous, in general. Still, the normal bundle is smooth, as a fiber bundle, and discontinuity of the group action can be carefully circumvented in the construction of the center manifold as a section to the normal bundle. For details of the construction, we refer to [Sa\&al99].

Palais coordinates Within the finite-dimensional relative center manifold $\mathcal{M}$, we now express the PDE dynamics (2.137) by an ODE, rather explicitly, using Palais coordinates $(g, v)$. See [Fi\&al96] for details. We choose a local Palais section $V$ in $\mathcal{M}$, transverse at $q_{*}$ to the group orbit $G q_{*}$. Then

$$
\begin{aligned}
G \times V & \rightarrow \mathcal{M} \\
(g, v) & \mapsto g\left(q_{*}+v\right)
\end{aligned}
$$

identifies $\mathcal{M}$ as a principal fiber bundle over $G q_{*}$, with fiber $V$ and structure group $H$. Indeed $(g, v)$ and $\left(g_{0}, v_{0}\right)$ parameterize the same point in $\mathcal{M}$ if, and only if,

$$
\begin{aligned}
g q_{*} & =g_{0} q_{*}, \quad \text { and } \\
g v & =g_{0} v_{0} .
\end{aligned}
$$

Therefore $g=g_{0} h$, for some $h \in H$, and thus $v_{0}=h v$. Hence

$$
\left(g_{0}, v_{0}\right)=\left(g h^{-1}, h v\right) .
$$

With respect to this free action of the isotropy $H$ on $(g, v) \in \mathcal{M}$, we indeed see that

$$
\mathcal{M}=(G \times V) / H
$$


is the orbit space of $H$. The action of $g_{0} \in G$ on $\mathcal{M}$ lifts to $(g, v) \in G \times V$ canonically:

$$
g_{0}(g, v):=\left(g_{0} g, v\right)
$$

in the above notation. Combining the actions (2.148), (2.150) we see how $\left(g_{0}, h\right) \in G \times H$ act by

$$
(g, h)(g, v):=\left(g_{0} g h^{-1}, h v\right)
$$

on $(g, v) \in G \times V$. We admit that our above presentation is simplistic due to the suggestive, but strictly speaking illegitimate, use of the notation $g\left(q_{*}+v\right)$ for an element in the transverse slice $g V$ to the group orbit $G q_{*}$ at $g q_{*}$. See [Fi\&al96] for full details. An alternative approach, focusing on the dynamics in the bundle $\mathcal{M}$ directly, was developed earlier by [Kr90] for compact groups $G$.

We now lift the flow from the relative center manifold $\mathcal{M}$ to the Palais coordinates $(g, v) \in G \times V$. By equivariance (2.150) alone, this flow must take the following form.

Theorem 2.5.2 [FißGal96] In Palais coordinates $(g, v)$, the G-equivariant flow (2.137) in the relative center manifold $\mathcal{M}$ takes the skew product form

$$
\begin{aligned}
\dot{g} & =g \mathbf{a}(v) \\
\dot{v} & =\Phi(v)
\end{aligned}
$$

for suitable vector fields $\Phi(v)$ on $V$ and $\mathbf{a}(v)$ on the Lie algebra $\operatorname{alg}(G)$. With respect to the compact isotropy $H$ of $q_{*}$ defined in (2.145), these vector fields transform according to

$$
\begin{aligned}
& \Phi(h v)=h \Phi(v) \\
& \mathbf{a}(h v)=h \mathbf{a}(v) h^{-1}
\end{aligned}
$$

For analogues of Theorem 2.5.2 for nonautonomous, periodically forced systems see [Sa\&al99]. For bifurcations from relatively periodic solutions, rather than relative equilibria, see [Sa\&al99, Wu\&al01].

The skew product form (2.152) is easily interpreted. Points $(g, v) \in G \times V$ with the same $v$-component mark points on the same $G$-orbit in $\mathcal{M}$, by the $G$-action (2.150). Therefore $v(t)$ indicates the shape of the original $x$-profile $u(t, \cdot) \in X$. The $G$-component $g(t)$, in contrast, indicates position $S(t)$ and phase $R(t)$, at least in the case $G=S E(m), g=(R, S)$, which is of primary interest here.

Spiral tip motion, Hopf meandering, and drift resonance To understand meandering spiral patterns, we rewrite (2.152) explicitly in terms of $g=\left(\mathrm{e}^{\mathrm{i} \alpha}, z\right) \in S E(2)$, with obvious complex notation for phase $R=\mathrm{e}^{\mathrm{i} \alpha}$ and position $S=z \in \mathbb{C}$. We immediately obtain the general form 


$$
\begin{aligned}
\dot{\alpha} & =\omega(v) \\
\dot{z} & =\mathrm{e}^{\mathrm{i} \alpha} \sigma(v) \\
\dot{v} & =\Phi(v) .
\end{aligned}
$$

We now argue that the position component $z=z(t)$ can be interpreted as tip position of a meandering spiral wave directly; see [FiTu98]. Let

$$
z_{*}: \mathcal{M} \rightarrow \mathbb{C}
$$

be any function which associates to a (nearly) spiral profile $u \in \mathcal{M} \subseteq \mathcal{U}$ near $G q_{*}$ a position $z_{*}(u) \in \mathbb{C}=\mathbb{R}^{2}$ of its "tip". Several such "tip" functions $z_{*}$ have been considered; see Figure 2.16. They all share the equivariance property

$$
z_{*}(g u)=g z_{*}(u)
$$

and hence lift to functions

$$
z_{*}: G \times V \rightarrow \mathbb{C}
$$

in Palais coordinates, which we again denote by $z_{*}$. A simple calculation then shows that

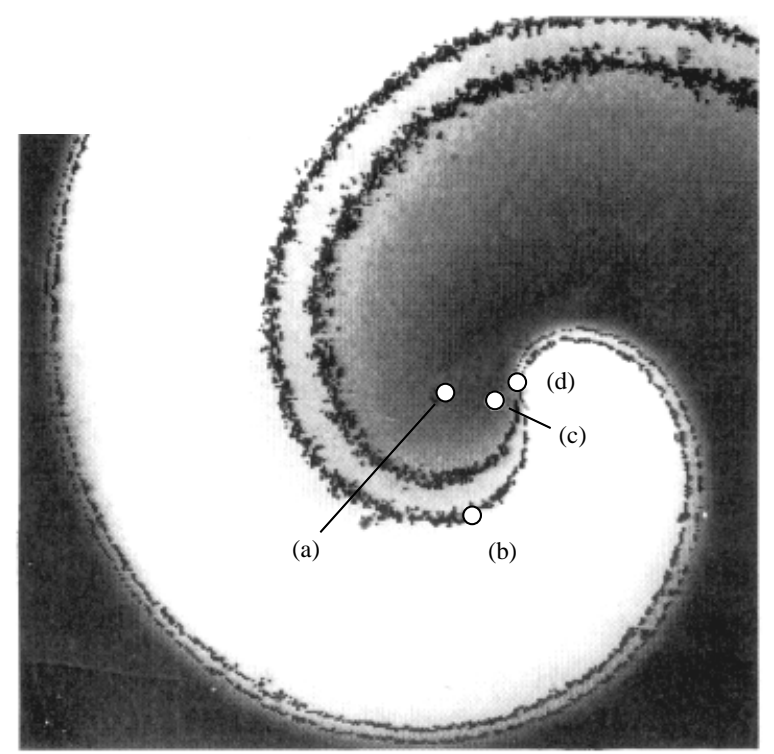

Fig. 2.17. "Tips" of a spiral wave pattern in the BZ reaction: (a) core center, (b) maximum curvature, (c) rotation center, (d) inflection point; courtesy of [MüZy94]. 


$$
\dot{z}=\mathrm{e}^{\mathrm{i} \alpha} \sigma_{*}(v),
$$

which takes the same form as the positional $z$-equation in (2.153). Only the precise form of the nonlinearity $\sigma$ depends on the precise form of the tip function $z_{*}$. Discussing the dynamics of skew product systems (2.154), in general, will therefore capture all the rather similar dynamics which are observed in the experimental literature - in spite of the varying definitions of a "tip". We therefore call $z(t)$ itself the tip of the spiral, henceforth.

Returning to a parameter dependent version of the skew product (2.154), we give a simple example. Consider shape variables $v \in \mathbb{R}^{2}$ undergoing Hopf bifurcation, due to a nontrivial pair of imaginary eigenvalues of the linearization at $q_{*}$, in corotating coordinates, crossing the imaginary axis transversely as the parameter $\mu$ increases. Then the shape variable $v=v(t)$ becomes periodic, say of Hopf frequency $\omega_{\mathrm{H}}$. Dividing (2.154) by the nonzero Euler multiplier $\omega(v)$ does not change the trajectory of the tip position $z \in \mathbb{C}$ and retains the abstract form of (2.154). We may therefore consider $\omega(v) \equiv \omega_{*}$ where $\omega_{*}$ indicates the normalized rotation frequency of the underlying rotating wave $q_{*}$, alias $v \equiv 0$.

The tip motion $z(t)$ is then obtained by simple integration. Fourier expansion

$$
\sigma(v(t))=\sum_{k \in \mathbb{Z}} \sigma_{k} \mathrm{e}^{\mathrm{i} k \omega_{*} t}
$$

provides the explicit expression

$$
\begin{aligned}
z(t) & =z_{0}+\int_{0}^{t} \mathrm{e}^{\mathrm{i} \omega_{*} t^{\prime}} \sigma\left(v\left(t^{\prime}\right)\right) d t^{\prime}= \\
& =z_{0}+\sum_{k \in \mathbb{Z}} \frac{\sigma_{k}}{\mathrm{i}\left(\omega_{*}+k \omega_{\mathrm{H}}\right)}\left(\mathrm{e}^{\mathrm{i}\left(\omega_{*}+k \omega_{\mathrm{H}}\right) t}-1\right)
\end{aligned}
$$

for Hopf frequencies $\omega_{\mathrm{H}}$ which are not an integer fraction of the normalized rotation frequency $\omega_{*}$. Clearly, (2.159) then describes a two-frequency quasiperiodic motion

$$
z(t)=\tilde{z}_{0}+\tilde{\sigma}(t) \mathrm{e}^{\mathrm{i} \omega_{*} t}
$$

with $\tilde{\sigma}$ periodic of frequency $\omega_{\mathrm{H}}$. For irrational frequency ratios $\omega_{*} / \omega_{\mathrm{H}}$, the tip position $z(t)$ densely fills an annulus around $\tilde{z}_{0}$ of inner and outer radius $\min |\tilde{\sigma}|$ and $\max |\tilde{\sigma}|$, respectively. At integer drift resonances

$$
k \omega_{\mathrm{H}}=\omega_{*}
$$

the tip motion $z(t)$ typically becomes unbounded, due to a nonzero drift term $\sigma_{-k} t$.

Such meandering and drifting motions have been observed experimentally, and numerically; see the discussion in Section 2.5.1. In view of work by [Mi91] 
in the Hamiltonian context, even the "slinky" spiral springs described by Love as far back as 1892 [Lo04], can be considered as an example for meandering. A first mathematical analysis of meandering spirals is due to [Wu96].

Relative normal forms With these interpretations in mind, we now proceed to sketch a normal form analysis of skew products (2.152), due to [FiTu98]. For standard normal form procedures, going back essentially to Poincaré, see the modern account in [Va89]. For vector fields like the shape dynamics $\dot{v}=\Phi(v)$, these procedures successively simplify finite order terms in the Taylor expansion

$$
\Phi(v)=M v+\cdots
$$

near the equilibrium $\Phi(0)=0$, by transformations

$$
\tilde{v}=\Psi(v)=v+\cdots .
$$

The action of the transformations $\Psi$ on $\Phi$ is given explicitly by the vector field pull-back

$$
\tilde{\Phi}(v)=\Psi^{\prime}\left(\Psi^{-1}(v)\right) \Phi\left(\Psi^{-1}(v)\right)
$$

Note linear dependence of $\tilde{\Phi}$ on $\Phi$. The standard normal form theorem asserts that nonlinear, finite order terms of $\Phi(v)$ in the range of the linear map $\Phi \mapsto \operatorname{ad}(M) \Phi$ can be eliminated, by suitable normal form transformations $\Psi$. Here

$$
((\operatorname{ad} M) \Phi)(v):=M \Phi(v)-\Phi^{\prime}(v) M v
$$

preserves polynomial order. A normal form of $\Phi$ thus amounts to a nonunique choice of a complement of range $(\operatorname{ad}(M))$. Nonremovable terms, in this procedure, are frequently called resonant.

For $G$-equivariant skew products $(2.152)$ we consider such a normal form procedure as already applied to the shape equation $\dot{v}=\Phi(v)$. The only remaining transformation then takes the general form $\tilde{g}=g_{0}(g, v)$. The requirement of $G$-equivariance (2.150) enforces the particular form $\dot{g}=g \mathbf{a}(v)$ in (2.154), which our normal form transformation $\Psi$ is supposed to preserve. Therefore our normal form transformation has to be of the form

$$
\tilde{g}=g g_{0}(v)
$$

with $g_{0}(0)=0$. Geometrically, such a transformation simply corresponds to the choice of a different slice $V$ to the group action in the center manifold $\mathcal{M}$; see $(2.146)$. by

The induced transformation of the Lie algebra factor $\mathbf{a}(v)$ is then given

$$
\tilde{\mathbf{a}}(v)=g_{0}^{-1}(v) \mathbf{a}(v) g_{0}(v)+g_{0}^{-1}(v) g_{0}^{\prime}(v) \Phi(v)
$$


with $g_{0}^{-1}$ denoting the group-inverse of $g_{0} \in G$. Again, ã depends (affine) linearly on a.

To formulate our normal form result, in this setting, we introduce some notation. Let $p$ enumerate the Jordan blocks of (not necessarily distinct) eigenvalues $\lambda_{p}$ of $M=\Phi^{\prime}(0)$, and let $1 \leq q \leq d_{p}$ enumerate a basis for each block. Then the shape variable $v$ possesses components $v_{p q}$ such that

$$
(M v)_{p q}=\lambda_{p} v_{p q}+v_{p, q+1}
$$

with the convention $v_{p, d_{p}+1}:=0$. With integer nonnegative multi-indices $\mathbf{k}=\left(k_{p q}\right)_{p q}$ we expand

$$
\tilde{g}=\sum_{\mathbf{k}} \mathbf{a}_{\mathbf{k}} v^{\mathbf{k}}
$$

with $\mathbf{a}_{0}=\mathbf{a}(0)$ unaffected by our transformation. We further decompose each $\mathbf{a}_{\mathbf{k}}$ spectrally by the adjoint action

$$
\begin{aligned}
\operatorname{ad}\left(\mathbf{a}_{0}\right): \operatorname{alg}(G) & \rightarrow \operatorname{alg}(G) \\
\mathbf{a} & \mapsto\left[\mathbf{a}_{0}, \mathbf{a}\right]
\end{aligned}
$$

on the Lie algebra $\operatorname{alg}(G)$. Let $\eta_{j}$ denote the distinct eigenvalues of this action, and spectrally decompose $\mathbf{a}_{\mathbf{k}}=\sum_{j} \mathbf{a}_{\mathbf{k}}^{j}$ accordingly.

Resonant terms, alias nonremovable terms, in our setting are those monomials $\mathbf{a}_{\mathbf{k}} v^{\mathbf{k}}$ in the expansion (2.169) which satisfy the resonance condition

$$
0=\eta_{j}+(\mathbf{k}, \lambda):=\eta_{j}+\sum_{p, q} k_{p q} \lambda_{p} .
$$

Note how this resonance condition captures an integer interaction between the eigenvalues $\lambda_{p}$ of the linearized shape dynamics $\dot{v}=M v$ and the eigenvalues of the unperturbed, exponential group dynamics $\dot{g}=g \mathbf{a}_{0}$ along the relative equilibrium $v=0$. We have already encountered such a resonance, with $\eta=\mathrm{i} \omega_{*}$ and $\lambda=\mathrm{i} \omega_{\mathrm{H}}$, as drift resonance of meandering spirals; see (2.161) and (2.143).

Theorem 2.5.3 [FiTu98] With the above assumptions and notations, there exists a normal form transformation (2.166) which preserves the skew product structure (2.152) and removes all nonresonant terms $\mathbf{a}_{\mathbf{k}}^{j}$ from $\mathbf{a}(v)$, for any finite order $1 \leq|\mathbf{k}|=\sum k_{p q}$. The $H$-equivariance (2.148), (2.153) of Palais coordinates can also be preserved. In the parameter-dependent case $\mathbf{a}=\mathbf{a}(\mu, v)$, the same elimination holds with coefficients $\mathbf{a}_{\mathbf{k}}(\mu)$ and with resonance defined at $\mu=0, v=0$.

We call any normal form reduction in the sense of Theorem 2.5.3 relative normal form. 
Relative Hopf resonance Instead of giving a proof of relative normal form Theorem 2.5.3, we illustrate some consequences for the Euclidean group $G=$ $S E(2)$, and for possible motions of spiral tips, by several examples. We begin with standard Hopf bifurcation at $\mu=\mu_{0}=0 \in \mathbb{R}$, as was already considered in $(2.154),(2.158)-(2.161)$. Normal form theory for Hopf bifurcation in $\dot{v}=\Phi(\mu, v)$ implies $\Phi=\Phi_{0}\left(\mu,|v|^{2}\right) v$, in complex notation for $v \in \mathbb{C}$. Therefore $|v| \equiv$ const., for periodic solutions $v(t)$. The Hopf eigenvalue is $\lambda=\mathrm{i} \omega_{\mathrm{H}} \neq 0$, of course. In the notation $(2.138)-(2.142)$ we have

$$
\mathbf{a}_{0}=\left(\mathbf{r}_{0}, \mathbf{s}_{0}\right)=\left(\mathrm{i} \omega_{*}, 0\right)
$$

for the rotating wave at $v=0$. Therefore $\eta \in \operatorname{spec} \mathbf{a}_{0}=\left\{ \pm \mathrm{i} \omega_{*}, 0\right\}$ provides a resonance (2.171) if, and only if,

$$
0=-\omega_{*}+k \omega_{\mathrm{H}}
$$

This is precisely the condition for drift resonance encountered in (2.161) above. For noninteger $\omega_{*} / \omega_{\mathrm{H}}$ we can eliminate all $v$-terms in $\dot{g}=g \mathbf{a}(\mu, v)$, and the normal form

$$
\dot{g}=\tilde{g} \mathbf{a}_{0}(\mu)
$$

yields purely exponential solutions $\tilde{g}(t)$ with frequency $\omega_{*}(\mu)$. Inverting the normal form transformation (2.166) then provides solutions

$$
g(t)=\tilde{g}(t) g_{0}(v(t))^{-1}
$$

which are clearly quasiperiodic, with $g_{0}(v(t))^{-1}$ contributing the Hopffrequency $\omega_{\mathrm{H}}$ of the shape dynamics, and $\tilde{g}(t)$ contributing the rotation frequency $\omega_{*}$ of pure unperturbed rotation. Since $g=\left(\mathrm{e}^{\mathrm{i} \alpha}, z\right)$, the tip motion $z(t)$ is also quasiperiodic. Of course the same arguments apply to general finite-dimensional Lie groups $G$. In particular, the case $G=S E(3)$ provides fascinating motions $z(t) \in \mathbb{R}^{3}$ of scroll rings (see Chapter 2.6) and vibrating arrows characterized by periodically pulsating straight propagation and superimposed sidewards meandering; see also [Fi\&al96].

Similar calculations allow us to analyze $k: 1$ drift resonance (2.173) of the frequencies $\omega_{*}: \omega_{\mathrm{H}}$. The nonresonant normal form for the tip motion $\tilde{z}(t)$ associated to the dynamics of $\tilde{g}(t)$ then becomes

$$
\dot{\tilde{z}}=\mathrm{e}^{\mathrm{i} \alpha} \sigma v^{k}
$$

where $\sigma=\sigma\left(\mu_{1}, \mu_{2}\right)$ depends on $\mu=\left(\mu_{1}, \mu_{2}\right)$ and $|v|^{2}$. For simplicity, we normalize $\mu_{2}=|v|^{2} \geq 0$ as the Hopf bifurcation parameter and let $\mu_{1}$ measure the detuning of the linearized Hopf frequency $\omega_{\mathrm{H}}\left(\mu_{1}\right)=\omega_{\mathrm{H}}(0)+\mu_{1}$ at the bifurcation line $\mu_{1} \in \mathbb{R}, \mu_{2}=0, v=0$. Then (2.176) implies

$$
\tilde{z}(t)=c-\sigma \mu_{2}^{k / 2}\left(\mathrm{e}^{-i k \mu_{1} t}-1\right) / i k \mu_{1}
$$


For the tip motion $z(t)$ in original coordinates, we have to account for the transformation $(2.166)$ by $g_{0}(v)=\left(\mathrm{e}^{\mathrm{i} \alpha_{0}(v)}, z_{0}(v)\right)$ of period (nearly) $2 \pi / \omega_{\mathrm{H}}$. In total we obtain a meandering tip motion of $z(t)$ along large circles of radius proportional to

$$
\mu_{2}^{k / 2} /\left|\mu_{1}\right|
$$

for $\mu_{2}>0$, which blow up at the drift resonance $\mu_{1}=0$. Strictly speaking we have suppressed terms in (2.176) which may not be in normal form. These terms however can be assumed to be perturbations of arbitrarily high finite order.

The superposition $g(t)=\tilde{g}(t) g_{0}(v(t))^{-1}$ in (2.175) also leads to curious tip motions right at bifurcation $\mu=\mu_{0}=0$, in these resonant cases. In nondegenerate normal form and for stable $v \equiv 0$, the shape component $v(t)$ converges to zero with $|v(t)| \sim t^{-1 / 2}$ and asymptotic phase frequency $\omega_{\mathrm{H}}$. For the tip motion $\tilde{z}(t)$ in normal form coordinates, $(2.176)$ provides a constant limit

$$
\tilde{z}_{\infty}=\lim _{t \rightarrow \infty} z(t)
$$

for orders $k \geq 3$ of the integer drift resonance $k=\omega_{*} / \omega_{\mathrm{H}}$. In terms of the true tip motion $z(t)$ this corresponds to a rotating wave limit, because $\tilde{z}_{\infty}$ can be eliminated by conjugation in $G=S E(2)$.

For $k=\omega_{*}: \omega_{\mathrm{H}}=2: 1$, however, $\tilde{z}(t)$ converges to a circular motion

$$
\tilde{z}(t) \sim c_{0}+c_{1} \exp (-i \tau(t))
$$

with constants $c_{0}, c_{1}$, asymptotically logarithmic phase $\tau(t)$, and decreasing angular velocity $\dot{\tau}(t) \sim 1 / t$. The tip motion $z(t)$ then corresponds to a meander with constant speed epicycles along a slowing base circle.

The 1:1 drift resonance $\omega_{*}=\omega_{\mathrm{H}}$, finally, gives rise to an unbounded motion (2.180) with logarithmic phase and nonconstant $c_{1} \sim \sqrt{t}$. All these results follow from just the normal form equation (2.176).

Clearly the analysis of relative mode interactions in the shape variable $v$ becomes feasible now. For an example involving Hopf-Hopf mode interactions of two complex eigenvalues $i \omega_{1}, i \omega_{2}$ see [As\&al01].

Relative Takens-Bogdanov bifurcation The case of nilpotent linearization $M=\Phi^{\prime}(0)$ in the shape dynamics $\dot{v}=\Phi(v)$, with trivial (relative) equilibrium $v=0$ contributes only eigenvalues $\lambda=0$ to the resonance condition (2.171). In normal form it turns out that

$$
\dot{\tilde{z}}=0
$$

because all terms in the translational $\tilde{z}$-equation are nonresonant. Indeed $\eta=\mathrm{i} \omega_{*}$ is generated only by the rotation frequency $\omega_{*}$ of the reference 
rotating wave; the zero eigenvalue of $\operatorname{ad}\left(\mathbf{a}_{0}\right)$ does not appear in the component $\tilde{z}$. In particular, any drift terms vanish beyond finite order.

A prime example, not observed experimentally so far, are the effects of a relative Takens-Bogdanov bifurcation in the shape variable $v \in \mathbb{R}^{2}$. For classical analysis see [Ta74, Bo81a, Bo81b, GuHo83]. From (2.154) we recall the tip motion

$$
\dot{z}=\mathrm{e}^{\mathrm{i} \omega_{*} t} \sigma(v(t)),
$$

again after rescaling time so that $\dot{\alpha} \equiv \omega_{*}$. If $v(t)$ is homoclinic to a hyperbolic relative equilibrium $v_{\infty}$, the resulting tip motion $z(t)$ satisfies

$$
z^{h}=[z(t)]_{-\infty}^{+\infty}=\int_{-\infty}^{+\infty} \mathrm{e}^{\mathrm{i}\left(\omega_{*} t+\alpha_{0}\right)} \sigma(v(t)) d t
$$

The integral converges if $\sigma\left(v_{\infty}\right)=0$ at the rotating wave $v_{\infty}$. Again this can be assumed after a conjugation in the group $G=S E(2)$. By hyperbolicity this implies exponential convergence $\sigma(v(t)) \rightarrow 0$, for $t \rightarrow \pm \infty$. The homoclinic shape dynamics $\Gamma=\{v(t) ; t \in \mathbb{R}\}$ thus typically implies heteroclinic tip dynamics, with heteroclinic tip shift $z^{h} \neq 0$.

Next suppose that the homoclinic orbit $\Gamma \subseteq \mathbb{R}^{2}$ is attracting from inside, due to a negative saddle quantity $\lambda_{1}+\lambda_{2}<0$ of the eigenvalues $\lambda_{1}<0<\lambda_{2}$ at its equilibrium $v=v_{\infty}$. Let $v(t)$ converge to $\Gamma$ from the inside. Then the passage times $t_{k}$ near $v=v_{\infty}$ grow geometrically like

$$
t_{k} \sim c_{1} \nu^{k}
$$

with $\nu=\left|\lambda_{1} / \lambda_{2}\right|>1$. Therefore the respective tip positions behave like

$$
z\left(t_{n+n_{0}}\right) \sim z\left(t_{n_{0}}\right)+\sum_{k=n_{0}+1}^{n} z^{h} \cdot \exp \left(\mathrm{i} \omega_{*} c_{1} \nu^{k}\right) .
$$

With respect to Lebesgue measure on $\omega_{*}$, this trigonometric power sum defines a random walk of $z\left(t_{n}\right)$ which can be rescaled to a Brownian motion of $z$. Indeed the increments are then only weakly coupled, for $k \rightarrow \infty$. See [BePh96] and, for explicit sample plots, Figure 2.18, with $\tilde{\omega}:=\omega_{*} c_{1}$.

We caution our reader on two points. First, the waiting times for each step of our random heteroclinic tip walk increase exponentially at rate $\log \nu>0$, with time. Observation of such a random walk may therefore take a very long time. Second, although the heteroclinic tip shift $z^{h}$ in (2.183) will typically be nonzero along homoclinic orbits $\Gamma$, it will be small beyond finite order in the Takens-Bogdanov case. See (2.181). Indeed the rotating wave phase $\exp (i \alpha(t))=\exp \left(\mathrm{i} \omega_{*} t\right)$ enters as a rapid forcing into the slow dynamics generated by the zero eigenvalues of the shape dynamics. In spite of these subtleties, the example shows clearly how a planar — and therefore nonchaotic - Poincaré-Bendixson type shape dynamics $v$ can cause complicated tip motions in the group component $\dot{g}=g a(v)$ of the skew product flow (2.152). 

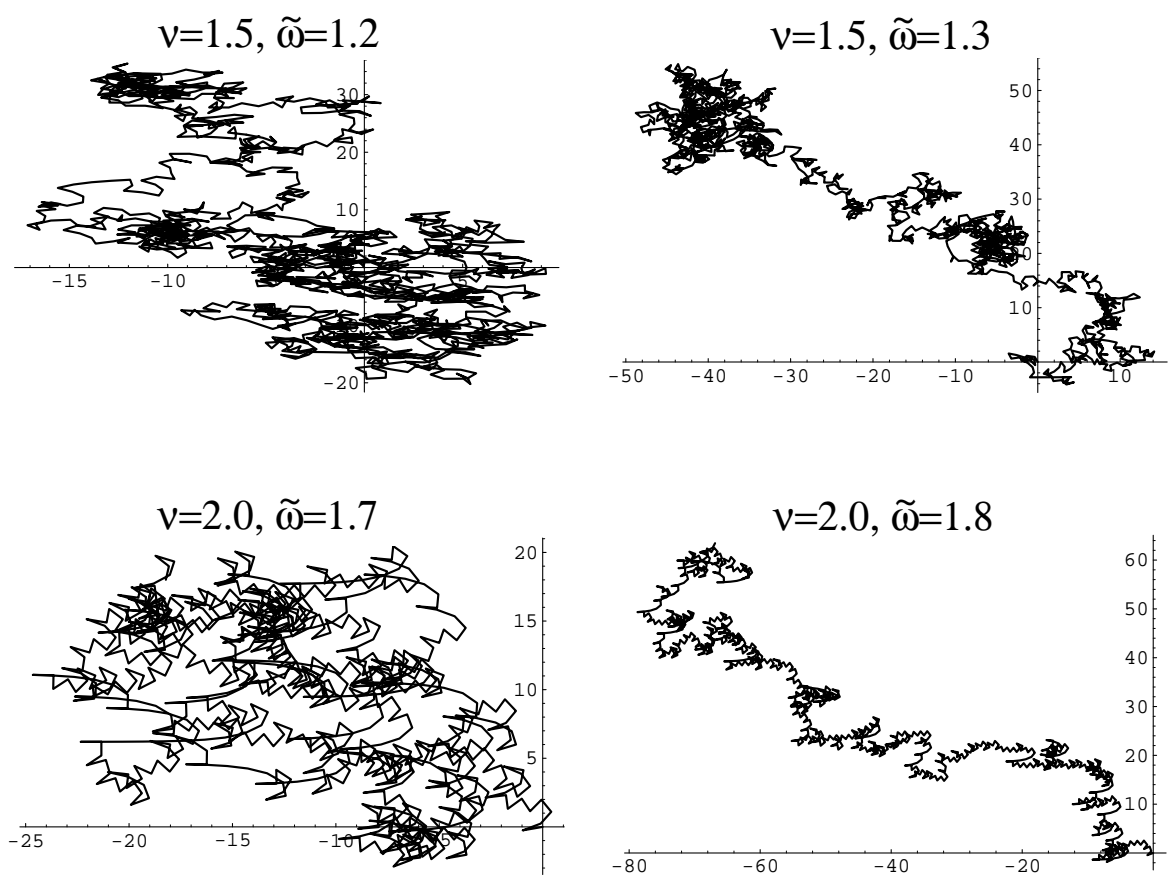

Fig. 2.18. Sample paths of Brownian tip motions due to asymptotically homoclinic shape dynamics.

\subsubsection{Spectra of Spiral Waves}

A major problem concerns a proper understanding of stability features of spiral waves. Even the notion of spectral stability turns out to be a very delicate problem. By means of a formal asymptotic matching procedure, Hagan [Ha82] showed that spiral waves in the complex Ginzburg-Lan"-dau equation (2.135) are likely to be stable. We give here a conceptual approach to the stability problem. We first characterize essential and absolute spectra of the spiral wave in terms of spectra of emitted wavetrains. We then describe the farfield shape of eigenfunctions to eigenvalues in the point spectrum in terms of the complex dispersion relation of the wavetrains.

The eigenvalue problem for spiral waves: core versus farfield As already pointed out in the discussion after Definition 2.4 .2 a variant of Proposition 2.3.1 holds for spiral waves. Fredholm indices are again determined by group velocities on Fredholm borders. In order to make a precise statement, we consider the eigenvalue problem for the linearization $\mathcal{L}_{*}$ at an Archimedean spiral $q_{*}(r, \psi)$. In corotating polar coordinates, as defined in $(2.127)$, the eigenvalue problem $\left(\mathcal{L}_{*}-\lambda\right) U=0$ becomes 


$$
\begin{aligned}
U_{r} & =V \\
V_{r} & =-\frac{1}{r} V-\frac{1}{r^{2}} \partial_{\psi \psi} U-D^{-1}\left(-\omega_{*} \partial_{\psi} U+f^{\prime}\left(q_{*}(r, \psi)\right) U-\lambda U\right) .
\end{aligned}
$$

As a first approach to the eigenvalue problem, we try to detect nontrivial bounded solutions of (2.186). An analogous consideration for the (formally) adjoint will then provide complete information about Fredholm properties such as the dimension of the kernel and the dimension of the cokernel.

The idea is to construct stable and unstable subspaces, containing solutions which are bounded in the farfield $r \rightarrow \infty$ and in the core $r \rightarrow 0$, respectively. From the dimension of these subspaces, we then read off the typical dimension of their intersection, which yields the dimension of the kernel. The dimension of the complement of their sum provides us with the dimension of the cokernel.

The core limit $r \rightarrow 0$ is again studied in the rescaled radial time $\tau=\log (r)$ :

$$
\begin{aligned}
U_{\tau} & =W \\
W_{\tau} & =-\partial_{\psi \psi} U-\mathrm{e}^{2 \tau} D^{-1}\left(-\omega_{*} \partial_{\psi} U+f^{\prime}\left(q_{*}(r, \psi)\right) U-\lambda U\right) .
\end{aligned}
$$

In the limit $\tau=-\infty$, corresponding to $r=0$, we formally obtain the autonomous, $\lambda$-independent system

$$
U_{\tau}=V, \quad V_{\tau}=-\partial_{\psi \psi} U
$$

The equation is readily solved by angular Fourier decomposition. We find solutions to the spatial eigenvalues $\nu= \pm \ell$,

$$
U(\tau, \psi)=U_{+}^{\ell} \mathrm{e}^{\mathrm{i} \ell \psi+\ell \tau}, \quad U(\tau, \psi)=U_{-}^{\ell} \mathrm{e}^{\mathrm{i} \ell \psi-\ell \tau},
$$

for $\ell \neq 0$ and

$$
U(\tau, \psi)=U_{+}^{0}, \quad U(\tau, \psi)=\tau U_{-}^{0},
$$

for the constant Fourier mode $\ell=0$. The vectors $U_{ \pm}^{\ell} \in \mathbb{C}^{N}$ are arbitrary. The condition $U_{-}^{\ell}=0$ for all $\ell$ defines the (infinite-dimensional) unstable subspace $E_{-}^{\mathrm{u}}$ of solutions which stay bounded in the limit $\tau \rightarrow-\infty$. Note that these asymptotic eigenspaces are independent of the spectral parameter $\lambda$. A generalization of the concept of exponential dichotomies from ordinary differential equations [Pa88, Co78] to the ill-posed, elliptic problem (2.187) allows us to extend the unstable subspaces to the non-autonomous system (2.187) as a $\tau$-dependent family of subspaces $E_{-}^{\mathrm{u}}(\tau ; \lambda)$; see [Pe\&al97, Sc98].

In the farfield limit $r \rightarrow \infty$, we formally obtain

$$
\begin{aligned}
& U_{r}=V \\
& V_{r}=-D^{-1}\left(-\omega_{*} \partial_{\psi} U+f^{\prime}\left(q_{\infty}\left(r-\frac{\psi}{k_{\infty}}\right)\right) U-\lambda U\right) .
\end{aligned}
$$

If we formally replace $\psi / \omega_{*} \mapsto t, r \mapsto x$, and $U \mapsto U \mathrm{e}^{-\lambda t}$, we obtain the linearization about the one-dimensional wavetrains 


$$
U_{t}=D U_{x x}+f^{\prime}\left(q_{\infty}\left(x-\frac{\omega}{k_{\infty}} t\right) U\right) .
$$

We have already discussed this equation in a comoving frame $\xi=x-\frac{\omega}{k_{\infty}} t$; see Section 2.3.1, (2.80).

Analogously to the unstable subspace $E_{-}^{\mathrm{u}}$ in the core region, we are going to determine the stable subspace $E_{+}^{\mathrm{s}}$ in the farfield equation (2.189). Since the farfield system is periodic in spatial "time" $r$, Floquet theory (2.81)(2.83) predicts that the stable subspaces are "time"-dependent and exponential growth or decay is governed by Floquet exponents $\nu$. In the next section, we determine these Floquet exponents, depending on the spectral parameter $\lambda$.

Spatial Floquet theory and the dispersion relation of wavetrains Our goal in this section is a characterization of exponential radial decay and growth properties of solutions to (2.189). The procedure is strongly reminiscent of Floquet theory (2.81)-(2.83) in Section 2.3.1, but the additional dependence on the angle $\psi$ introduces some complications.

To eliminate explicit dependence on the angle $\psi$ in (2.189), we exploit the Archimedean shape of the spiral by introducing "Archimedean coordinates". We replace $\psi$ by $\xi=r-\left(\psi / k_{\infty}\right)$, and find

$$
\begin{aligned}
& U_{r}=-\partial_{\xi} U+V \\
& V_{r}=-\partial_{\xi} V-D^{-1}\left(\frac{\omega_{*}}{k_{\infty}} \partial_{\xi} U+f^{\prime}\left(q_{\infty}(\xi)\right) U-\lambda U\right) .
\end{aligned}
$$

Dependence of $q_{\infty}$ on $\xi$ is $2 \pi / k_{\infty}$-periodic. The equation is now autonomous in $r$, and solutions are of the general form $U=\mathrm{e}^{-\nu r} \hat{U}(\xi)$, where $\hat{U}$ solves the boundary-value problem

$$
D\left(\frac{\mathrm{d}}{\mathrm{d} \xi}-\nu\right)^{2} \hat{U}+\frac{\omega_{*}}{k_{\infty}} \frac{\mathrm{d}}{\mathrm{d} \xi} \hat{U}+f^{\prime}\left(q_{\infty}(\xi)\right) \hat{U}=\lambda \hat{U},
$$

with periodic boundary conditions on $\left[0,2 \pi / k_{\infty}\right]$. Note that $(2.192)$ is equivalent to the boundary-value problem (2.125) for the dispersion relation of the wavetrains. To simplify (2.192) further, we introduce $\tilde{U}(\xi):=\mathrm{e}^{\nu \xi} \hat{U}(\xi)$. This yields

$$
D \tilde{U}_{\xi \xi}+\frac{\omega_{*}}{k_{\infty}} \tilde{U}_{\xi}+f^{\prime}\left(q_{\infty}(\xi)\right) \tilde{U}=\tilde{\lambda} \tilde{U}
$$

with $\tilde{\lambda}:=\lambda-\left(\omega_{*} \nu / k_{\infty}\right)$. Boundary conditions are now of Floquet type

$$
\tilde{U}(0)=\mathrm{e}^{2 \pi \nu / k_{\infty}} \tilde{U}\left(2 \pi / k_{\infty}\right)
$$

induced from the $2 \pi / k_{\infty}$-periodic boundary conditions for $\hat{U}$.

The dimension of the stable subspace $E_{+}^{\text {s }}$ for (2.189) changes whenever a Floquet exponent $\nu$ crosses the imaginary axis, as $\lambda$ is varied. We therefore focus on solutions to (2.193), (2.194), with $\nu=\mathrm{i} k$, purely imaginary, first. 
In analogy to the one-dimensional discussion in Section 2.3.1, we call $k$ the Bloch wavenumber.

Nontrivial solutions to the boundary-value problem (2.192),(2.194) can be found from the dispersion relation

$$
d_{\text {co }}(\tilde{\lambda}, \nu)=\operatorname{det}\left(\Phi(\tilde{\lambda})-\mathrm{e}^{2 \pi \nu / k_{\infty}}\right)=0
$$

where $\Phi(\lambda)$ denotes the period- $2 \pi / k_{\infty}$ map to $(2.193)$, rewritten as a firstorder differential equation; see (2.83). Substituting $\tilde{\lambda}=\lambda-\left(\omega_{*} \nu / k_{\infty}\right)$ we obtain

$$
d_{\mathrm{st}}(\tilde{\lambda}, \nu)=\operatorname{det}\left(\Phi\left(\lambda-\left(\omega_{*} \nu / k_{\infty}\right)\right)-\mathrm{e}^{2 \pi \nu / k_{\infty}}\right)=0 .
$$

If we fix $\operatorname{Im} \nu \in\left[0, k_{\infty}\right)$, we find $N$ solution $\lambda^{\ell}(\nu)$. The Floquet shift $\nu \mapsto$ $\nu+\mathrm{i} k_{\infty}$ gives us the vertically shifted spectral curves

$$
\lambda^{\ell}(\nu)+\mathrm{i} \omega_{*} \mathbb{Z}
$$

Note that the associated group velocity

$$
c_{\mathrm{g}, j}(\mathrm{i} k):=\operatorname{Im}\left(\frac{\mathrm{d} \lambda_{j}(\mathrm{i} k)}{\mathrm{d} k}\right)=\operatorname{Im}\left(\frac{\mathrm{d} \tilde{\lambda}_{j}(\mathrm{i} k)}{\mathrm{d} k}\right)+c_{\infty},
$$

corresponds precisely to our previous definition (2.126) of the group velocity of the wavetrains.

Restricting to $\nu=\mathrm{i} k, k \in \mathbb{R}$, we obtain the spectral curves $\Gamma^{\ell}=$ $\left\{\lambda^{\ell}(\mathrm{i} k) ; k \in \mathbb{R}\right\}$. We emphasize that together with a fixed spectral curve $\Gamma^{\ell}$, there is the countable collection of vertically shifted (not necessarily disjoint) curves $\Gamma^{\ell}+\mathrm{i} \omega_{*} \mathbb{Z}$. Comparing (2.190) with (2.193), we find that these curves consist of the continua of Floquet exponents for the linearized period map for the one-dimensional wavetrains.

For an illustration, we deviate from the general setup and discuss a specific example. Assume that the wavenumber $k_{\infty}$ of the emitted wavetrains is small. More specifically, assume that on their very large interval of periodicity, $\xi \in$ $\left[0,2 \pi / k_{\infty}\right]$, the wavetrains resemble a pulse solution $q_{*}(\xi),\left|q_{*}(\xi)\right| \rightarrow 0$ for $|\xi| \rightarrow \infty$. We then refer to the wavetrains as pulse trains. Spectral properties of pulsetrains are determined by interaction of individual pulses, as we shall explain next. In particular, we can compute the Floquet spectrum of the pulse trains from certain geometric properties of a single pulse, alone. First consider the pulsetrains and the pulse in a comoving frame. Then the spectrum of the pulsetrains converges to the spectrum of the single pulse [SaSc01c]. Close to the (critical) zero-eigenvalue of the pulse with translation eigenfunction $q_{*}^{\prime}(\xi)$, the spectrum of the pulsetrains consists of a small circle of essential spectrum

$$
\tilde{\lambda}(\nu)=a\left(k_{\infty}\right)\left(\mathrm{e}^{2 \pi \nu / k_{\infty}}-1\right)+\mathrm{o}\left(\left|a\left(k_{\infty}\right)\right|\right), \quad \nu=\mathrm{i} k \in \mathrm{i} \mathbb{R},
$$


see Figure 2.19(a). The coefficient $a$ is given as $a\left(k_{\infty}\right)=M \mathrm{e}^{-\delta / k_{\infty}}$. The constant $\delta>0$ is the weakest exponential decay rate at the tails of the individual pulse. The coefficient $M$ measures the (signed) speed of crossing of stable and unstable manifolds of the origin in the travelling-wave equation

$$
D q^{\prime \prime}+c q^{\prime}+f(q)=0
$$

when the speed $c$ is varied. Note that $a\left(k_{\infty}\right) \rightarrow 0$, exponentially, for $k_{\infty} \rightarrow 0$. This reflects that the interaction strength between individual pulses decreases exponentially with their distance.

Spectral stability corresponds to $a\left(k_{\infty}\right)<0$. The crucial coefficient $M$ turns out to be positive in the classical FitzHugh-Nagumo equation.

In a comoving coordinate frame of speed $c_{\infty}$, we have to add the speed of the frame to the group velocity and find

$$
\lambda(\nu)=a(k)\left(\mathrm{e}^{2 \pi \nu / k_{\infty}}-1\right)+c_{\infty} \nu+\mathrm{o}(|a(k)|), \quad \nu=\mathrm{i} k \in \mathrm{i} \mathbb{R}
$$

see Figure 2.19(b). The result is a vertically periodic epicycloid, winding along the imaginary axis, with tangencies at $i \omega_{*} \mathbb{Z}$.

a)

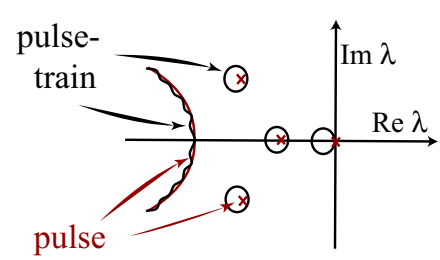

b)

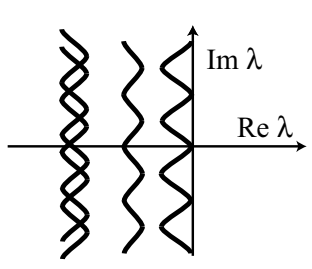

Fig. 2.19. The spectrum of wavetrains of weakly interacting pulses in the steady frame (a), and the Floquet exponents in the comoving frame (b).

Relative Morse indices and essential spectra of spiral waves In the spirit of Proposition 2.3.1, the oriented curves $\Gamma^{\ell}$ determine the Fredholm index of the linearization about a spiral wave, see (2.186). However, Proposition 2.3.2 has to be reformulated since both Morse indices $i_{ \pm}$at $\tau=-\infty$ (alias $r=0$ ) and $r=\infty$ are infinite. Proposition 2.3.2 remains valid if both Morse indices $i_{ \pm}$are normalized to finite numbers, the relative Morse indices, which then enter formula (2.72) for the Fredholm index; see [SaSc01d] for details. A similar idea has been used repeatedly in the construction of Floer homology in various variational problems; see for example [RoSa95].

Since the asymptotic equation (2.188) in the core region, $\tau \rightarrow-\infty$, does not depend on $\lambda$, we can fix the Morse index in $-\infty$ and (arbitrarily) normalize to $i_{-}=0$. We show next, how to define $i_{+}(\lambda)=: i_{\text {Morse }}(\lambda)$, such 
that Proposition 2.3.1 carries over to to linearizations at spiral waves: the Fredholm index of $\mathcal{L}_{*}$ is again given by the difference of the Morse indices

$$
i\left(\mathcal{L}_{*}-\lambda\right)=i_{-}-i_{+}
$$

We solve the dispersion relation (2.196) for the radial Floquet exponent $\nu$ as a function of $\lambda$. For fixed $\lambda$, there exists an infinite number of spatial Floquet exponents $\nu_{j}$, with $0 \leq \operatorname{Im} \nu<2 \pi / k_{\infty}$, which solve (2.196). Just like in the example of the heat equation (2.91), the real parts of the $\nu$ are unbounded on the positive and negative axis. Instead of counting unstable eigenvalues, we therefore count differences between numbers of unstable eigenvalues in different regions of the complex plane $\lambda \in \mathbb{C}$.

Consider the (open) complement of the spectral curves in the complex plane

$$
\mathcal{U}=\mathbb{C} \backslash \bigcup_{\ell=1}^{N}\left(\Gamma^{\ell}+\mathrm{i} \omega_{*} \mathbb{Z}\right) .
$$

For all $\lambda \in \mathcal{U}$, the asymptotic equation in the farfield, (2.189) is hyperbolic, that is, there are no purely imaginary solutions $\nu=\mathrm{i} k$ to the dispersion relation (2.196). In consequence, the Morse index $i_{\text {Morse }}$ is constant on connected components of $\mathcal{U}$.

In the connected component $\mathcal{U}_{\infty}$ of $\mathcal{U}$ containing $\operatorname{Re} \lambda \rightarrow+\infty$, the Fredholm index is zero. Indeed, the linearization $\mathcal{L}_{*}-\lambda$ is invertible, there, since the parabolic reaction-diffusion system is well-posed. In view of (2.199), we define $i_{\text {Morse }}(\lambda) \equiv 0$ in $\mathcal{U}_{\infty}$.

If we now move $\lambda$ from one connected component of $\mathcal{U}$ to another, we cross a spectral curve $\Gamma^{\ell}+\mathrm{i} \ell^{\prime} \omega_{*}$. For $\lambda \in \Gamma^{\ell}+\mathrm{i} \ell^{\prime} \omega_{*}$, a Floquet exponent $\nu$ is located on the imaginary axis and hyperbolicity fails. The Morse index of $\mathcal{L}_{*}-\lambda$ changes by one when $\lambda$ is varied across this single spectral curve. In order to determine the direction of change, we orient the curves $\Gamma^{\ell}$ such that vertical slope corresponds to positive group velocity, similarly to Proposition 2.3.1. With this orientation, the Floquet exponent $-\nu$ (recall the Ansatz $U \sim \exp (-\nu r))$ moves from $\operatorname{Re} \nu<0$ to $\operatorname{Re} \nu>0$ when $\lambda$ crosses the spectral curve from left to right; see also Lemma 2.3.3. In particular, the (relative) number of Floquet exponents in $\operatorname{Re} \nu<0$ decreases by one each time we cross spectral curves from left to right. We call this (relative) number of Floquet exponents in $\operatorname{Re} \nu<0$ the relative Morse index $i_{\text {Morse }}$. This index is now defined in $\mathcal{U}$ via a $\lambda$-homotopy from $\mathcal{U}_{\infty}$ to any point in $\mathcal{U}$, counting signed crossings of the oriented curves $\Gamma^{\ell}+i \ell^{\prime} \omega_{*}$ during the homotopy.

Proposition 2.5.4 The operator $\mathcal{L}_{*}-\lambda$, defined in (2.127), considered as an unbounded operator on $L^{2}\left(\mathbb{R}^{2}\right)$ is Fredholm if, and only if $\lambda \notin \Gamma^{\ell}$, that is, if $\lambda$ belongs to the complement $\mathcal{U}$ of the set of Floquet exponents of the emitted wavetrains. The Fredholm index is given by

$$
i\left(\mathcal{L}_{*}-\lambda\right)=-i_{\text {Morse }}(\lambda)
$$


where the relative Morse index $i_{\text {Morse }}$ was introduced above. In particular, the Fredholm index increases by one when crossing any of the oriented Fredholm borders $\Gamma^{\ell}+\mathrm{i} \ell^{\prime} \omega_{*}$ from left to right.

In the remainder of this section, we are going to derive a "typical" picture of the critical spectrum of a spiral wave. First notice that for $\lambda=0$, the derivative of the wave train $U=q_{\infty}^{\prime}$ provides a trivial solution to $(2.193-$ 2.194) with $\nu=0$. If we assume that $\lambda=0$ is simple as a root of the dispersion relation (2.196), then there is a unique spectral curve $\Gamma^{0}=\lambda^{0}(\mathrm{i} k)$, contained in the essential spectrum, that touches the imaginary axis in $\lambda=0$. Since the positive group velocity of the emitted wavetrain does not vanish, the curve $\Gamma^{0}$ is not tangent to the real axis, see Definition 2.4.2. Because the spectrum of $\mathcal{L}_{*}$ is invariant under complex conjugation, the curve $\Gamma^{0}$ necessarily possesses a vertical tangent at $\lambda=0$. Typically, the tangency will be quadratic. If the wave trains are spectrally stable, $\Gamma^{0}$ is the rightmost Fredholm border in the complex plane. In $\operatorname{Re} \lambda>0$, the Fredholm index of $\mathcal{L}_{*}$ therefore is 0 . Since the group velocity is positive, the region $\operatorname{Re} \lambda>0$ is to the right of the Fredholm border $\Gamma^{0}$. To the left of this curve $\Gamma^{0}$, in $\operatorname{Re} \lambda<0$, the Fredholm index of the linearization is -1 , by Proposition 2.5.4. See Figure 2.20 for an illustration of the resulting spectral picture.

Positive group velocity was encoded in Definition 2.4.2 by means of exponential weights. Consider the linearization in $L_{\eta}^{2},(2.128)$, with radial weight $\eta<0$ close to zero. Then the weight pushes the critical spectral curve $\Gamma$ into the stable, left complex half plane. In other words, the exponentially decaying weight penalizes the outward transport; see Section 2.3.1 and Figure 2.20. Note that translation $\left(\partial_{x_{1}}+\mathrm{i} \partial_{x_{2}}\right) q_{*}$ and angular derivative $\partial_{\psi} q_{*}$ are bounded. They therefore contribute to the kernel in the space $L_{\eta}^{2}$, which allows for exponential growth.
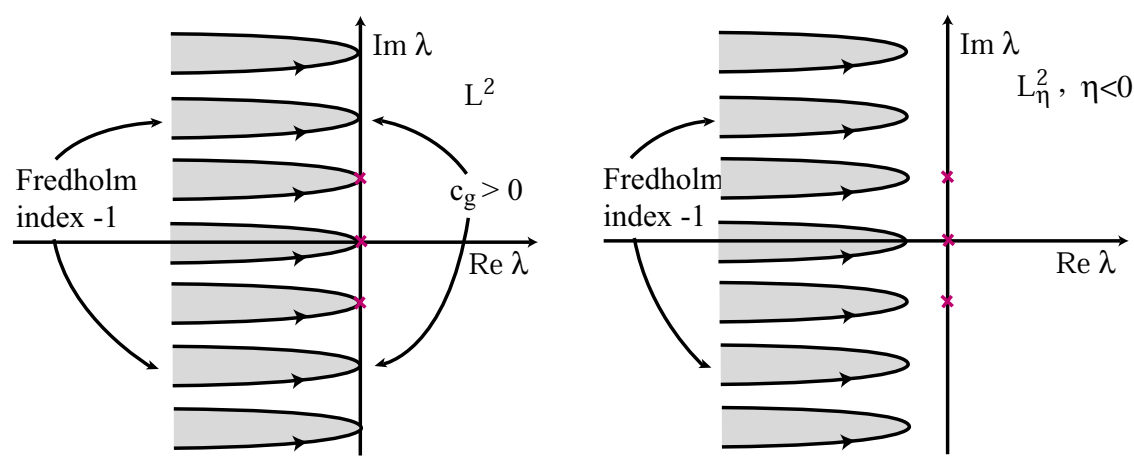

Fig. 2.20. Typical spectrum of the linearization about a spiral wave with Fredholm indices of the linearization in $L^{2}$ and in $L_{\eta}^{2}$, for eta $<0$. 
Along the imaginary axis, the spectral picture is periodic with period $i \omega_{*}$. The essential spectrum touches the imaginary axis at all points $i \omega_{*} \mathbb{Z}$. Since the wavetrains emitted from the center have a positive group velocity in the radial direction, this implies that the Fredholm index of $\mathcal{L}_{*}-\lambda$ is negative, $i\left(\mathcal{L}_{*}-\lambda\right)=-1$, for $\lambda$ to the left of all these critical spectral curves $\lambda^{\ell^{\prime}}(\mathrm{i} k)$, $\lambda^{\ell^{\prime}}(0)=\mathrm{i} \ell^{\prime} \omega_{*},$.

The abundance of critical spectrum, in the form of infinitely many tangencies of continuous spectrum at the imaginary axis, seems to suggest that spiral waves are extremely sensitive to perturbations. However, spiral waves in experiments appear to be very stable objects in many systems! In the next section, we will find a partial explanation for this apparent contradiction.

Absolute spectra of spiral waves We have seen in Section 2.3.3 how stability properties of waves on bounded domains are captured by the absolute spectrum rather than the essential spectrum. In this section, we carry out the analogous construction to define the absolute spectrum of a spiral wave. We then state a convergence theorem for spectra of linearizations along spiral waves on large bounded disks, as the diameter of the disk tends to infinity.

We return to the boundary-value problem (2.193),(2.194), with dispersion relation (2.196). For $\lambda$ fixed, there is a countable family of spatial Floquet exponents $\nu_{j}=\nu_{j}(\lambda)$ with $0 \leq \operatorname{Im} \nu_{j}(\lambda)<k_{\infty}$. The normalization eliminates trivial shifts by $\mathrm{i} k_{\infty}$. Each of these Floquet exponents can be seen to be of finite multiplicity as a root of the analytic dispersion relation (2.196). We order the Floquet exponents with increasing real part, repeated by multiplicity

$\ldots \leq \operatorname{Re} \nu_{-j-1} \leq \operatorname{Re} \nu_{-j} \leq \ldots \leq \operatorname{Re} \nu_{-1} \leq \operatorname{Re} \nu_{1} \leq \ldots \leq \operatorname{Re} \nu_{j-1} \leq \operatorname{Re} \nu_{j} \leq \ldots$

Floquet exponents $\nu$ depend on $\lambda$ and the labeling might be discontinuous in $\lambda$. We omitted the label 0 in the counting, for convenience. As already pointed out, the sequence is bi-infinite, that is, $\nu_{j} \rightarrow \pm \infty$ for $j \rightarrow \pm \infty$.

At this stage, we could shift all labels, $j \mapsto j+j^{\prime}$ and find an equivalent labeling. However, the Morse index considerations in the Section 2.5.3 suggest a normalization at $\operatorname{Re} \lambda=+\infty$. Indeed, for $\operatorname{Re} \lambda$ positive and large, all $\nu_{j}(\lambda)$ have nonvanishing real part and we can write with a consistent splitting

$$
\ldots \leq \operatorname{Re} \nu_{-1}(\lambda)<0<\operatorname{Re} \nu_{1}(\lambda) \leq \ldots
$$

Recall that the $\nu_{j}(\lambda)$ change sign when $\lambda$ crosses one of the Fredholm borders $\Gamma^{\ell}$.

With normalization (2.201), we continue the labeling for all values of $\lambda$. We define the absolute spectrum of $\mathcal{L}_{*}$ through

$$
\operatorname{spec}_{\text {abs }} \mathcal{L}_{*}:=\left\{\lambda ; \operatorname{Re} \nu_{-1}(\lambda)=\operatorname{Re} \nu_{1}(\lambda)\right\} .
$$

As a first conclusion from the definition we note that the absolute spectrum is a subset of the essential spectrum of $\mathcal{L}_{*}$. Indeed, either $\operatorname{Re} \nu_{-1}(\lambda)=0$ 
and the relative Morse index jumps at $\lambda \in \operatorname{spec}_{\text {abs }} \mathcal{L}_{*}$, or else $\operatorname{Re} \nu_{-1}(\lambda)=$ $\operatorname{Re} \nu_{1}(\lambda) \neq 0$ and $i_{\text {Morse }}(\lambda) \neq 0$; see Proposition 2.5.4.

Note next that for $\lambda$ in the complement of the absolute spectrum, $\mathcal{L}_{*}-\lambda$ is Fredholm of index zero in an exponentially weighted space $L_{\eta}^{2}$, with radial weight $\eta=\eta(\lambda)$ satisfying

$$
\cdots \leq \operatorname{Re} \nu_{-1}(\lambda)<-\eta(\lambda)<\operatorname{Re} \nu_{1}(\lambda) \leq \ldots
$$

Similar to the definition in Section 2.3.3, we can define the extended point spectrum $\operatorname{spec}_{\text {expt }} \mathcal{L}_{*}$ and the boundary spectrum $\operatorname{spec}_{\text {bdy }} \mathcal{L}_{*}$.

We now characterize the limiting behavior of the spectra on bounded disks. As in the case of Theorem 2.3.6, we omit a number of technical assumptions in the statement of the following theorem.

Theorem 2.5.5 [SaSc00c, SaSc00d] Let $\mathcal{L}_{*}$ denote the linearization operator (2.127) considered on $L^{2}\left(\mathbb{R}^{2}\right)$. Let $\mathcal{L}_{*, R}$ denote the corresponding operator on a disk, $|x| \leq R$, equipped with typical mixed boundary conditions. Then the spectrum of $\mathcal{L}_{*, R}$ converges as $R \rightarrow \infty$,

$$
\operatorname{spec} \mathcal{L}_{*, R} \longrightarrow \operatorname{spec}_{\text {abs }}\left(\mathcal{L}_{*}\right) \cup \operatorname{spec}_{\text {expt }}\left(\mathcal{L}_{*}\right) \cup \operatorname{spec}_{\text {bdy }}\left(\mathcal{L}_{*}\right) .
$$

Convergence is uniform on bounded subsets of the complex plane in the symmetric Hausdorff distance. Moreover, multiplicity of the eigenvalues is preserved: the number of eigenvalues, counted with multiplicity, in any fixed open neighborhood of any point $\lambda_{*} \in \operatorname{spec}_{\text {abs }}\left(\mathcal{L}_{*}\right)$ converges to infinity as $R \rightarrow \infty$. Multiplicities in neighborhoods of points $\lambda_{*} \in \operatorname{spec}_{\text {expt }}\left(\mathcal{L}_{*}\right), \operatorname{spec}_{\mathrm{pt}}\left(\mathcal{L}_{*}\right)$, $\operatorname{spec}_{\mathrm{bdy}}\left(\mathcal{L}_{*}\right)$ stabilize and convergence of eigenvalues is of exponential rate in $R$, there.

For transverse Archimedean spiral waves, the rightmost curve $\Gamma^{0}=\left\{\lambda^{0}(\mathrm{i} k)\right\}$ of the essential spectrum is simple, that is, only a single Floquet exponent $\nu=$ $\nu_{1}(\lambda)$ is located on the imaginary axis. In particular, the absolute spectrum does not contain $\lambda=0$. If $\Gamma^{0} \cap \mathrm{i} \mathbb{R}=\{0\}$, and all other curves $\Gamma^{\ell}$ are contained in $\operatorname{Re} \lambda<0$, then the absolute spectrum of the spiral wave is stable, i.e. contained in $\operatorname{Re} \lambda<0$. Since the Fredholm borders $\Gamma^{\ell}$ consist of the Floquet exponents of the one-dimensional wavetrains, (2.190),(2.189), stability of the absolute spectrum is equivalent to one-dimensional stability of the wavetrains.

This explains, on a spectral level, why spirals waves appear to be very stable, despite the abundance of critical spectrum winding up the imaginary axis. If the spiral is considered as a solution in a bounded disk, only finitely many eigenvalues are close to criticality in a bounded domain - uniformly in the size $R$ of the domain, if the absolute spectrum is stable.

We may say slightly more about the spectral properties of spirals. Rotation and translation of the spiral cause 0 and $\pm \mathrm{i} \omega_{*}$ to be in the spectrum: eigenfunctions are given explicitly by $\partial_{\psi} q_{*}(r, \psi)$, and $\mathrm{e}^{ \pm \mathrm{i} \psi}\left(\partial_{r} \pm \frac{\mathrm{i}}{r} \partial_{\psi}\right) q_{*}(r, \psi)$, respectively. In particular, these eigenfunctions are bounded functions of 
$(r, \psi)$. Considering the linearization in $L_{\eta}^{2}$ with radial weight $\eta<0$ small, the linearization $\mathcal{L}_{*}$ is Fredholm with index zero, by Definition 2.4.2, due to the positive group velocity of the wavetrains. Because $L_{\eta}^{2}$ allows for (small) exponential growth, radially, translation and rotation eigenfunctions contribute to the kernel. In other words, $\lambda=0$ and $\lambda= \pm \mathrm{i} \omega_{*}$ belong to the extended point spectrum and therefore, these eigenvalues stay exponentially close to the imaginary axis when truncating to a bounded domain.

Conversely, consider an Archimedean spirals with negative group velocity, violating Definition 2.4.2 of a transverse Archimedean spiral. Then the essential spectrum is pushed into the stable complex plane by an exponential weight $\exp (\eta r)$ with $\eta>0$ ! As opposed to Figure 2.20, translation and rotation eigenvalues now do not belong to the extended point spectrum of $\mathcal{L}_{*}$, since the exponential weight requires exponential decrease of eigenfunctions. As a consequence, Euclidean equivariance does not imply critical spectrum of spirals with negative group velocity. Skew-product descriptions of tip motions as described in Section 2.5.2 is not possible, and even the abstract notion of a tip, (2.155) is not well defined in this case.

Point spectrum and the shape of eigenfunctions In the following, we will show how simple, temporal point spectrum $\lambda_{0}$ of the linearization $\mathcal{L}_{*}$ along an Archimedean spiral $q_{*}\left(r, \varphi-\omega_{*} t\right) \rightarrow q_{\infty}\left(k_{\infty} r-\left(\varphi-\omega_{*} t\right)\right)$ gives rise to super-spiral patterns, near resonances $\lambda_{0} \sim \mathrm{i} \ell \omega_{*}$. See expansion (2.208), below. We prepare the resonance consideration with a more general result, which describes the radial asymptotics of the eigenfunction $u_{0}(r, \psi)$, related to any simple, temporal eigenvalue $\lambda_{0}$; see Proposition 2.5.6.

Consider a simple eigenvalue $\lambda_{0}$ in the extended point spectrum of $\mathcal{L}_{*}$, as defined in Section 2.5.3, with eigenfunction $u_{0}(r, \psi)$. Consider the roots $\nu_{j}^{0}=\nu_{j}^{0}\left(\lambda_{0}\right)$ of the dispersion relation $(2.196)$, associated with the asymptotic wavetrains, and the ordering $(2.200)$

$$
\begin{aligned}
& \ldots \leq \operatorname{Re} \nu_{-j-1}^{0} \leq \operatorname{Re} \nu_{-j}^{0} \leq \ldots \\
& \leq \operatorname{Re} \nu_{-1}^{0}<\operatorname{Re} \nu_{1}^{0} \leq \ldots \leq \operatorname{Re} \nu_{j-1}^{0} \leq \operatorname{Re} \nu_{j}^{0} \leq \ldots
\end{aligned}
$$

Note that $\operatorname{Re} \nu_{-1}^{0}<\operatorname{Re} \nu_{1}^{0}$ since $\lambda_{0}$ does not belong to the absolute spectrum (2.202). Assume in addition that $\nu_{1}^{0}$ is a simple root of the dispersion relation (2.196).

Proposition 2.5.6 [SaSc01a] In the setting of the preceding paragraph, the asymptotic shape of $u_{0}$ for $r \rightarrow \infty$ is given by

$$
\left|u_{0}(r, \psi)-u_{\infty}\left(r-\left(\psi / k_{\infty}\right)\right) \mathrm{e}^{-\nu_{1} r}\right|=\mathrm{o}\left(\mathrm{e}^{-\nu_{1} r}\right)
$$

uniformly in $\psi$. Here $u_{\infty}(\xi)$ is a spatially periodic solution to the Floquet problem (2.192) of the radially asymptotic wavetrain $q_{\infty}$ with $\lambda=\lambda_{0}$.

Consider nonzero $u_{\infty}$, in Proposition 2.5.6. Then exponential radial decay or growth of $u_{0}$ is determined by the real part of the first Floquet exponent $\nu_{1}$ of 
the asymptotic wavetrain $q_{\infty}$. Also the temporal eigenvalue $\lambda_{0}$ and the radial decay rate $\nu_{1}^{0}$ of its eigenfunction $u_{0}$ are related by the dispersion relation (2.196) of the wavetrain.

We now turn to the particular case of a near-resonant, simple, imaginary eigenvalue

$$
\lambda_{0}=\mathrm{i} \ell \omega_{*}+\mathrm{i} \delta \omega,
$$

where $\delta \omega$ indicates a small deviation from the $\ell: 1$-resonance with the spiral frequency $\omega_{*}$.

At resonance, $\delta \omega=0$, we obtain explicitly

$$
u_{\infty}\left(r-\psi / k_{\infty}\right)=\mathrm{e}^{-\mathrm{i} \ell \psi} q_{\infty}^{\prime}\left(r-\psi / k_{\infty}\right),
$$

simply because $q_{\infty}^{\prime}(\xi)$ is the Floquet solution of the trivial Floquet exponent $\nu_{1}=0$ and due to the Floquet shift (2.197).

Slightly off resonance, that is, for small nonzero $\delta \omega$, we observe associated perturbations $\delta u_{\infty}$, of the radially asymptotic Floquet eigenfunction $u_{\infty}(\xi)$, and $\delta \nu_{1}$, of the corresponding Floquet exponent. In view of Proposition 2.5.6, $\delta u_{\infty}$ remains $2 \pi$-periodic, as $u_{\infty}$ itself, and has little effect on the observed pattern. A variation of $\operatorname{Re} \delta \nu_{1} \neq 0$ modifies the radial exponential decay or growth of the eigenfunction $u_{0}$. From the discussion in Section 2.5.3 on crossing of Floquet exponents near Fredholm borders, Proposition 2.5.4, we predict exponential growth in the Fredholm index -1 region and exponential decay in the Fredholm index 0 region; see Figure 2.20. However, $\operatorname{Re} \delta \nu_{1} \neq 0$ does not influence the $r$-asymptotic shape of the pattern. Only $\operatorname{Im} \delta \nu_{1} \neq$ 0 interferes with the underlying periodicity of the primary spiral pattern, producing super-spiral patterns; see Figure 2.22. More specifically,

$$
\begin{aligned}
u_{0}(r, \psi) & \sim \mathrm{e}^{-\nu_{1}^{0} r} u_{\infty}\left(r-\psi / k_{\infty}\right)=\mathrm{e}^{-\delta \nu_{1} r} \mathrm{e}^{-\mathrm{i} \ell \psi} \tilde{q}_{\infty}^{\prime}(\xi) \\
& =\mathrm{e}^{-\left(\operatorname{Re} \delta \nu_{1}\right) r} \mathrm{e}^{-\mathrm{i} \ell \psi} \tilde{q}_{\infty}^{\prime}(\xi) \mathrm{e}^{-\mathrm{i}\left(\operatorname{Im} \delta \nu_{1}\right) r}
\end{aligned}
$$

Here, $\tilde{q}_{\infty}^{\prime}(\xi)$ is a small, but $2 \pi$-periodic perturbation of the wavetrain eigenfunction $q_{\infty}^{\prime}(\xi)$, and $\sim$ indicates identity up to a term of order $\mathrm{o}\left(\mathrm{e}^{-\nu_{1}^{0} r}\right)$, for $r \rightarrow \infty$. The term $\mathrm{e}^{-\mathrm{i} \ell \psi}$ again accounts for the Floquet shift of $u_{\infty}$. Since $\nu_{1}=0+\delta \nu_{1}$ satisfies the dispersion relation $(2.196)$, the definition of the group velocity $c_{\mathrm{g}}(2.198)$ implies

$$
\operatorname{Im} \delta \nu_{1}=\frac{\operatorname{Im} \delta \nu_{1}}{\operatorname{Im} \delta \lambda_{1}} \cdot \operatorname{Im} \delta \lambda_{1}=\frac{\delta \omega_{*}}{c_{\mathrm{g}}},
$$

in the resonant limit $\delta \omega \rightarrow 0$. Substituting (2.207) into (2.206), and returning to the original coordinates $r, \varphi=\psi+\omega_{*} t$, and time $t$, the eigenfunction generates a perturbation

$$
u_{\text {pert }}(t, r, \varphi) \sim \mathrm{e}^{-\left(\operatorname{Re} \delta \nu_{1}\right) r} \cdot \mathrm{e}^{\mathrm{i}\left(\ell \varphi-\left(r-c_{\mathrm{g}} t\right) \delta \omega / c_{\mathrm{g}}\right)} \cdot \tilde{q}_{\infty}^{\prime}\left(r-\left(\varphi-\omega_{*} t\right) / k_{\infty}\right),
$$

again for $r \rightarrow \infty$, and in the limit $\delta \omega \rightarrow 0$. 
The eigenfunction shape $u_{\text {pert }}$, corresponding to a near-resonant temporal eigenvalue $\lambda_{0}=\mathrm{i} \ell \omega_{*}+\mathrm{i} \delta \omega$, produces the pattern of a superspiral, for large $r$. Indeed, consider fixed $t$. Then $u_{\text {pert }}$ is an $\ell$-armed spiral, due to the term $\exp \left(\mathrm{i} \ell \varphi-\delta \omega r / c_{\mathrm{g}}\right)$. The superspiral is Archimedean with large radial wavelength $2 \pi c_{\mathrm{g}} / \delta \omega$, for $\delta \omega \rightarrow 0$. The superspirals propagate in radial direction with speed $c_{\mathrm{g}}$ and rotate with the slow frequency $\delta \omega$.

In the experimentally observed pattern $q_{*}+\varepsilon u_{\text {pert }}$, the superspiral modulations manifest themselves in a phase shift of the emitted wavetrains

$$
q_{*}+\varepsilon u_{\text {pert }} \sim q_{\infty}\left(r-\left(\varphi-\omega_{*} t\right) / k_{\infty}+\mathrm{e}^{-\left(\operatorname{Re} \delta \nu_{1}\right) r} \cos \left(\ell \varphi-\left(r-c_{\mathrm{g}} t\right) \delta \omega / c_{\mathrm{g}}\right)\right) .
$$

Here we exploited that $\tilde{q}_{\infty}^{\prime} \sim q_{\infty}^{\prime}$ in $(2.208)$ amounts to a phase shift of the emitted wavetrains.

\subsubsection{Comparison with experiments}

We explain the onset of spatio-temporal patterns and certain characteristics of incipient spiral wave instabilities, referring to the linear spectral analysis of the preceding section. In particular, we discuss the possibility of point spectrum crossing the imaginary axis, Section 2.5.4, and essential spectrum causing an instability, Section 2.5.4.

Meander instabilities Meander instabilities are caused by critical point spectrum, emerging from the essential spectrum; see Figure 2.21. In any disk of finite radius $R$, the essential spectrum disappears and five critical eigenvalues remain close to the imaginary axis. The influence of the boundary of the domain on the location of point spectrum is exponentially small for large disk radius $R$. Actually, also the nonlinear terms of the vector field on the center-manifold converge with exponential rate, as the size $R$ of the disk tends to infinity $[\mathrm{SaSc02b}$. In the limit, we recover the $S E(2)$-equivariant skewproduct description (2.152). The equations on the center manifold in a finite disk are therefore a small perturbation of the $S E(2)$-invariant skew-product system, discussed in Section 2.5.2 - as long as the tip stays far enough from the disk boundary.

Therefore, in any large disk, we recover the skew-product dynamics, even though a dynamical reduction in the unbounded domain is prohibited by the presence of essential spectrum.

Dynamics and tip motion are complemented in the experiments with striking super-patterns in the farfield, when sufficiently large spirals are observed. As a consequence of center manifold reduction, the spatial patterns are superpositions of the critical eigenfunctions, to leading order in the bifurcation parameter. For two purely imaginary eigenvalues close to resonance $\mathrm{i} \omega_{\mathrm{H}} \sim \ell \mathrm{i} \omega_{*}$ we have computed the shape; see (2.209). Predictions coincide well with direct simulations of a specific reaction-diffusion system, based on Barkley's code EZSpiral [Do\&al97]; see Figure 2.22. For marginally stable wavetrains, 


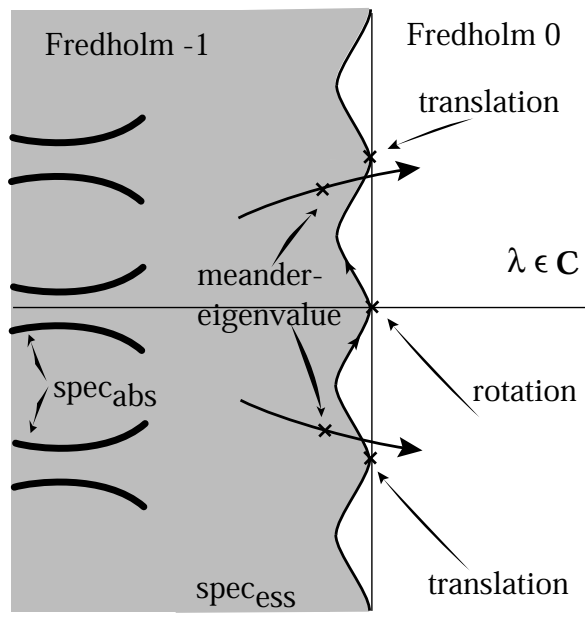

Fig. 2.21. Schematic plot of the meander eigenvalues popping out of the Fredholm index -1 region close to instability.

the essential spectrum touches the imaginary axis only at $i \ell \omega_{*}, \ell \in \mathbb{Z}$, with quadratic tangency. From the previous section, we conclude that, off resonance, super-patterns $u_{\text {pert }}$ resulting from the instability will be strongly localized in the farfield (2.208). In particular, at the higher drift-resonances, $\omega_{*}=\ell \omega_{\mathrm{H}}, \ell \geq 2$, the super-patterns will be strongly localized. At the inverse resonances, $\ell \omega_{*}=\omega_{\mathrm{H}}, \ell \geq 2$ where the motion of the tip stays bounded, we do predict prominent, non-localized super-patterns in the form of weakly curved $\ell$-armed super-spirals (2.208). The orientation of the super-spirals changes when the Hopf eigenvalue crosses through resonance. At transition, the superpattern divides the domain in sectors. At the 1:1-resonance, a particularity occurs, since $\mathrm{i} \omega_{*}$ is now algebraically double in the extended point spectrum. Drift resonance coincides with the emergence of strongly pronounced superspirals. In [SaSc01a], it is shown that the principal eigenfunction is computed from integration of the eigenfunction in the radial direction. Its amplitude grows linearly with the radius $r$, such that the super-pattern exhibits an asymptotically $r$-independent shift of the wavenumber of the wavetrains; see the drift picture in Figure 2.22.

Experimentally, these striking superstructures, have first been observed in a biological system [Pe\&al91]. An explanation based on a curvature description of the emitted pulse-trains, in the spirit of Section 2.4.1, has been attempted in [Pe\&al93]. The simplest phenomenological explanation evokes the picture of a point source, alias the tip, emitting wavetrains. Variations of the local wavenumber are due to a Doppler-effect caused by the two-frequency motion of the spiral tip. Spatial decay as well as the correct speed of prop- 
agation are not predicted from these formal descriptions, which neglect the interaction between individual emitted pulses.
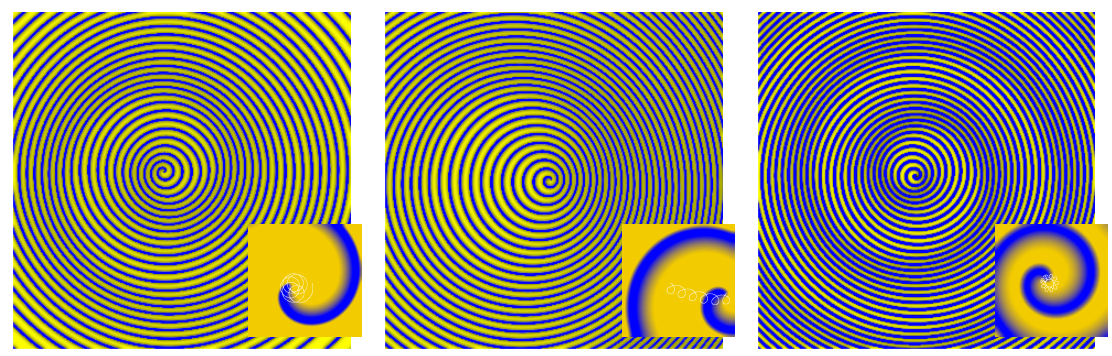

Fig. 2.22. Farfield patterns and tip motion in case of inward meander, drift, and outward meander. The large pictures show the superspiral-shaped deformations of the primary spiral. The small inlets show, on a smaller spatial scale, the tip motion. See Plate ?? in the Appendix for a version of this figure in colour.

The spectral assumptions of point spectrum crossing the axis were confirmed numerically by Barkley; see [Ba92]. In a disk, he found five critical eigenvalues at bifurcation. The eigenvalues from translation were extremely close to the axis, although the domain size was moderate, exhibiting about one turn of the spiral. This confirms the rapid (exponential) convergence of point spectrum in bounded domains of increasing size $R$; see Theorem 2.3.6. The numerically observed change of the growth behavior of the amplitude of the eigenfunction is in good agreement with the predictions from Section 2.5.3. Indeed, close to resonance, (2.208) predicts that the asymptotic behavior close to bifurcation changes: from exponential radial growth - when the eigenvalue is still located in the Fredholm index -1 region — to weak exponential radial decay at bifurcation.

Farfield and core breakup Farfield breakup is caused by essential spectrum crossing the imaginary axis [BäOr99, SaSc00d, ToKn98]. Mostly, a convective Eckhaus instability is observed, with unstable wavenumbers close to the wavenumber of the wavetrains, $\operatorname{Im} \nu \sim k_{\infty}$. In the stable regime, the boundary curve $\Gamma$ of the essential spectrum touches the imaginary axis at $i \omega_{*} \mathbb{Z}$ with quadratic tangency from the left. At criticality, the tangency is of fourth order. In the unstable regime, the tangency is from the right; see Figure 2.23. The absolute spectrum, however, remains confined in the open left half plane, close to this instability threshold. The group velocities (2.198) on the critical spectral curve $\Gamma$ are always directed outwards close to $\lambda=0$, by transversality of the spiral; see Definition 2.4.2. Therefore, the Eckhaus unstable modes inherit a positive group velocity, as well. In any large bounded disk, the Eckhaus instability remains transient, as long as the absolute spectrum stays confined to the left complex half plane. This was confirmed in direct simulations, see [SaSc00d]. We computed essential spectra of the wavetrains 
from the boundary-value problem $(2.193),(2.194)$ following the dependence of a nontrivial solution $u(\xi ; k), \lambda(\mathrm{i} k)$ on the Bloch wavenumber $k$. The absolute spectrum was computed by a similar path-following procedure. Instability thresholds coincided well with the observations in direct simulations. In the convectively unstable regime, stability depends crucially on the size of the domain and the magnitude of the perturbation. The sensitivity is caused by the subcritical nature of the Eckhaus instability in the given parameter regime. Qualitatively, the sensitivity mimics the one-dimensional nonlinear advection-diffusion problem, addressed in Proposition 2.3.7: the minimal amount $\delta^{\mathrm{u}}$ of a perturbation needed to cause breakup decays rapidly with the size $R$ of the domain. In numerical simulations, again based on Barkley's code EZSpiral [Do\&al97], the domain contained approximately 20 wavelengths; see Figure 2.25.

The absolute instability, if close enough to the convective instability, is produced by eigenfunctions which grow exponentially towards the boundary of the domain; see Figure 2.23. Their shape is again determined from the complex dispersion relation (2.196), which predicts prominent, exponentially growing super-spirals (2.208). Note that these super-spirals resemble the farfield pattern in the meander instability. The most striking difference is their growth towards the boundary. However, we emphasize that super-spirals in meandering and in farfield breakup occur for completely different reasons. In the farfield breakup, the motion of the tip is a regular one-frequency motion.
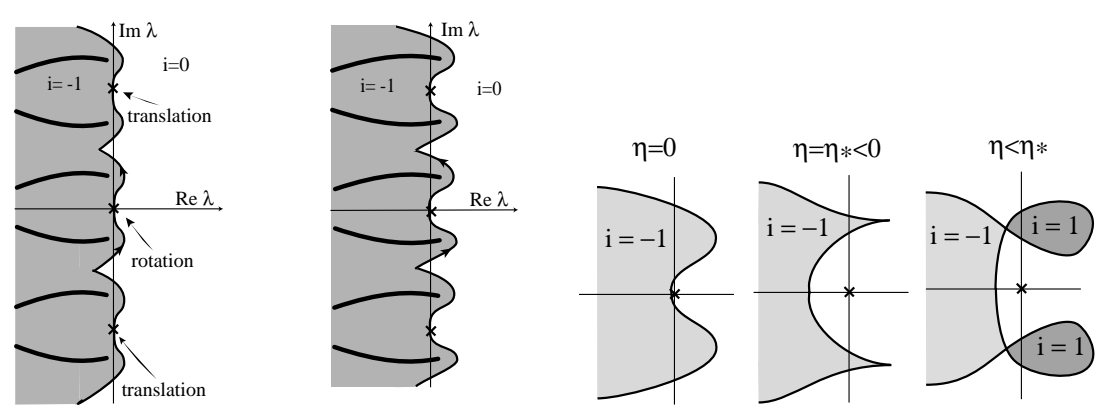

Fig. 2.23. Schematic plot of essential and absolute spectra crossing the axis successively at farfield breakup.

We discuss core breakup, next. Numerical computations of essential and absolute spectra indicate that parts of the critical curve $\Gamma$ change slope to create group velocities directed towards the center of the spiral. The shape of the boundary curve of the essential spectrum becomes more complicated. In 
Figure 2.24, two scenarios are suggested, that produce negative group velocities. Recall that the sign of the group velocity determined the vertical slope of the oriented curve $\Gamma^{\ell}$. The onset of the second scenario in Figure 2.24 has been found in $[\mathrm{SaSc00d}]$ in a parameter regime close to core-breakup. In direct simulations of a nonlinear reaction-diffusion system, super-spirals appear before breakup, but are strongly localized. Much more detailed numerical investigation is necessary, however, to clarify the structure of this instability.
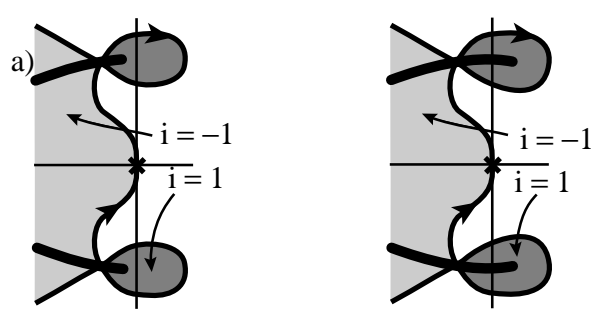

b)
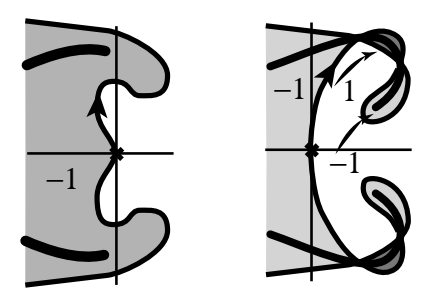

Fig. 2.24. Two scenarios (a), (b) of essential and absolute spectra crossing the axis successively at core-breakup.

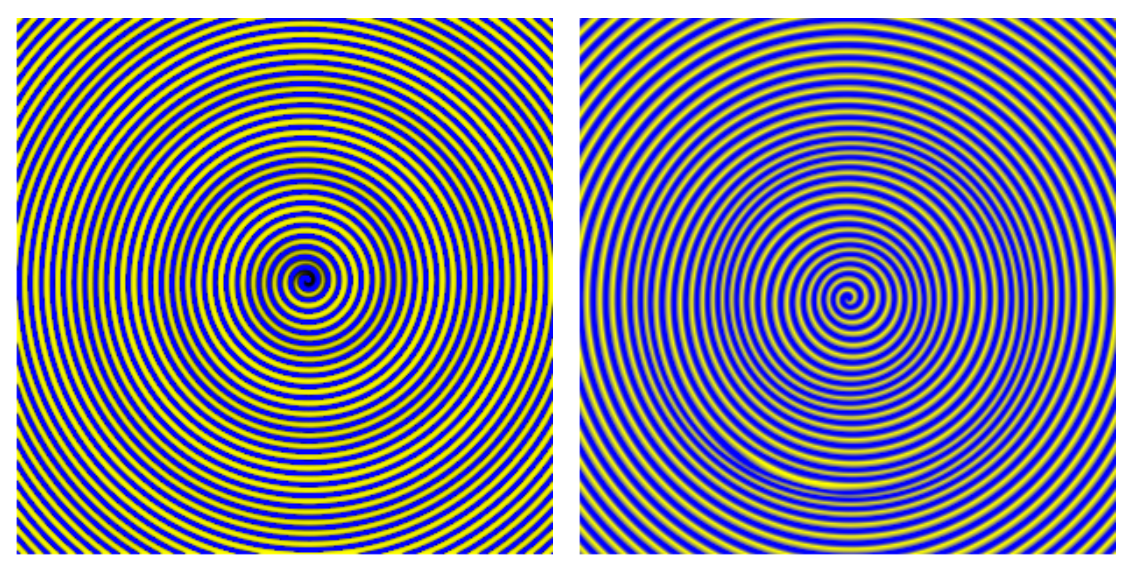

Fig. 2.25. Farfield patterns in case of farfield- and core-breakup. See Plate ?? in the Appendix for a version of this figure in colour.

The final states in farfield versus core breakup are quite different. Collision of wavetrains leads to the formation of new spirals. In the case of farfield breakup, the size of the various spiral domains remains large, close to the onset of instability, leading to a moderately complicated dynamical behavior. In the opposite case of core breakup, new spirals immediately break up, 
close to the core, and the final state is extremely complicated right after the instability has set in; see Figure 2.26.
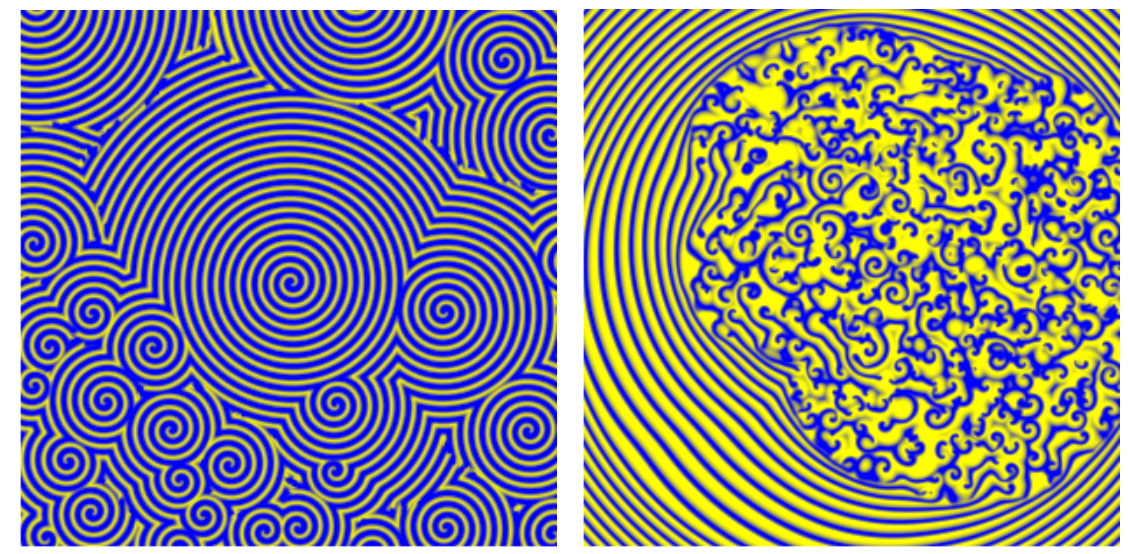

Fig. 2.26. Final state after farfield and core breakup. See Plate ?? in the Appendix for a version of this figure in colour.

Meander instabilities in the presence of convective farfield instabilities enhance the effect of the instability. From the spectral analysis in Section 2.5.3 we predict the meander eigenfunctions to be exponentially growing. Indeed, at instability, they are embedded in the Fredholm index -1 region. Whereas the unstable essential spectrum disappears in bounded domains, the (extended) point spectrum still reproduces the effect of the essential spectrum. Indeed exponential radial growth of eigenfunctions is governed by the dispersion relation (2.196). In the simplistic phenomenological description by a Doppler effect, the meander movement of the spiral tip is amplified and transported by the wavetrains. Increasing the size of the domain, we find breakup in the farfield already for moderate amplitudes of the meander motion.

\subsection{Three Space Dimensions: Scroll Waves}

\subsubsection{Filaments, Scrolls, and Twists}

In this chapter we consider reaction-diffusion systems of $N=2$ equations in $m=3$ space dimensions,

$$
u_{t}=D \Delta u+f(t, x, u) .
$$

Here $u=\left(u_{1}, u_{2}\right) \in \mathbb{R}^{2}$ and $x$ is in a bounded domain $\Omega \subset \mathbb{R}^{3}$, say with Neumann boundary conditions. Let $f$ be smooth. A motivating prototype is the singularly perturbed FitzHugh-Nagumo-like system with nonlinearity 


$$
f=\left(\begin{array}{c}
\varepsilon^{-1} u_{1}\left(1-u_{1}\right)\left(u_{1}-\left(u_{2}+b\right) / a\right) \\
u_{1}-u_{2}
\end{array}\right)
$$

of excitable media type, for small $\varepsilon>0$. See Section 2.4.1, and (2.114). For simplicity of notation, we shift $u=\left(u_{1}, u_{2}\right)$ by suitable constants, in each component, so that the value $u=0$ becomes a value inside the singular homoclinic pulse cycle of Figure 2.16(a).

Spirals, tips, and Brouwer degree In $m=2$ space dimensions, $\Omega \subset \mathbb{R}^{2}$, the pre-images

$$
z_{*}(t)=(u(t, \cdot))^{-1}(0)
$$

may serve as a viable definition for a spiral tip in the sense of (2.155), (2.156). Both experimental evidence and numerical simulations suggest, in fact, that nondegenerate zeros $z_{*}$ of $u(t, \cdot)$ are typically accompanied by local spiral wave patterns of (2.211) with rotation direction determined by the local Brouwer degree (see for example [De85, Du\&al85, Ze93] for some background):

$$
\operatorname{deg}_{l o c} u(t, \cdot):=\operatorname{sign} \operatorname{det} u_{x}\left(t, z_{*}(t)\right)
$$

at $x=z_{*}(t)$. So pronounced is this effect, indeed, that Winfree has proposed to model excitable media by only a phase variable $\tilde{u}=\tilde{u}(t, x) \in S^{1}$, with appropriate time evolution [Wi01, Wi87, Wi95]. Here the circle $S^{1}$ can model the homoclinic pulse cycle of Figure 2.16(a), or else just an abstract local "clock". Mathematically, the correspondence to our PDE approach can be achieved by simply defining $\tilde{u}:=u /|u|$. The local Brouwer degree (2.213) coincides with the winding number of $\tilde{u}$, restricted to a small circle around the singularity of $\tilde{u}$ at $x=z_{*}$. Sometimes this local degree is called "topological charge" of the singularities of spirals. Conservation of "charge", as time $t$ evolves, then corresponds to homotopy invariance of Brouwer degree: only anti-rotating spiral pairs can annihilate each other.

The restriction to $\tilde{u} \in S^{1}$ unfortunately produces a phase singularity of undefined $\tilde{u}$ at the spiral tips, where $u=0$. Since we are moreover interested in collisions of such singularities, not only in two but also in three space dimensions, we therefore favor the reaction-diffusion description by smooth spatial profiles $u(t, \cdot) \in \mathbb{R}^{2}$ over the phase fields $\tilde{u}(t, \cdot) \in S^{1}$.

Scroll waves, filaments, and twists Returning to three-dimensional $x \in$ $\Omega \subset \mathbb{R}^{3}$, the preimages

$$
\tau \mapsto z=z(t, \tau) \in(u(t, \cdot))^{-1}(0) \subseteq \Omega
$$

become differentiable curves $z(t, \cdot)$, at least as long as zero is a regular value of $u(t, \cdot)$. We call these curves (tip) filaments. Numerical observations in excitable media exhibit spiral wave patterns, when restricting the spatial profile 
$u(t, \cdot)$ to a local, two-dimensional section $S^{\perp} \subset \Omega$ transverse to the filament curve $z(t, \cdot)$ at $z_{*}=z\left(t, \tau_{0}\right)$. Note, that we may always define an orientation of $S^{\perp}$ such that

$$
\left.\operatorname{deg}_{l o c} u(t, \cdot)\right|_{S^{\perp}}=+1
$$

at $x=z_{*}$. Requesting the pair $\left(S^{\perp}, z_{\tau}\right)$ of $S^{\perp}$ and the filament tangent $z_{\tau}$ to be oriented by the right-hand rule thus defines a unique filament orientation all along each filament curve $\tau \mapsto z(t, \tau)$.

A scroll wave is the wave pattern arising in a tubular neighborhood of a filament curve. By the excitable media paradigm, the scroll wave consists of a stack of spirals in the transverse sections $S$ along the filament. See Figure 2.27 for a reproduction of Panfilov and Winfree's original drawings [PaWi85]. We emphasize that our mathematical discussion of scroll waves neither proves nor relies on the validity of the "stack of spirals" picture. Our results below will hold in a general framework of reaction-diffusion systems. In particular, they are not limited to a singular perturbation setting. It is nevertheless very useful to refer to Winfree's paradigm, for visual motivation and guidance.

For closed filaments $z(t, \tau), \tau \in S^{1}$, a twist of the oriented filament can be defined as follows. Choose $\delta>0$ small enough and consider the closed curve

$$
\tau \mapsto \tilde{z}(t, \tau) \subseteq(u(t, \cdot))^{-1}(\delta, 0)
$$

of the pre-image of $u_{1}=\delta, u_{2}=0$ near $z(t, \tau)$, following the orientation of $z$ itself. Geometrically, the curve $\tilde{z}(t, \cdot)$ indicates the local phases of the spiral motions in the stacked sections $S^{\perp}$. Then we call

$$
\operatorname{twist}(z(t, \cdot)):=\operatorname{lk}(\tilde{z}(t, \cdot), z(t, \cdot))
$$

the twist of the filament $z(t, \cdot)$. Here $\mathrm{lk}$ denotes the linking number of oriented curves, as explained below. A twisted scroll possesses a filament $z$ with nonzero twist: the curves $z(t, \cdot)$ and $\tilde{z}(t, \cdot)$ are linked.

To define the linking number $\mathrm{lk}(\tilde{z}, z)$ of two closed oriented disjoint curves $\tilde{z}=\tilde{z}(\tau), z=z(\tau), \tau \in S^{1}$, we consider a suitable planar projection such that crossings of the two curves are transverse and distinct, in projection. See Figure 2.28, for examples of such projected crossings, indicating overand under-crossings in the original three-dimensional view. Denoting the oriented unit tangent vectors of the over- and under-crossing curves by $\mathbf{t}_{1}, \mathbf{t}_{2}$, respectively, we associate a sign

$$
\varepsilon_{j}:=\operatorname{sign} \operatorname{det}\left(\mathbf{t}_{1}, \mathbf{t}_{2}\right)= \pm 1
$$

to each crossing $j$. Then

$$
\operatorname{lk}(\tilde{z}, z):=\frac{1}{2} \sum_{j} \varepsilon_{j}
$$



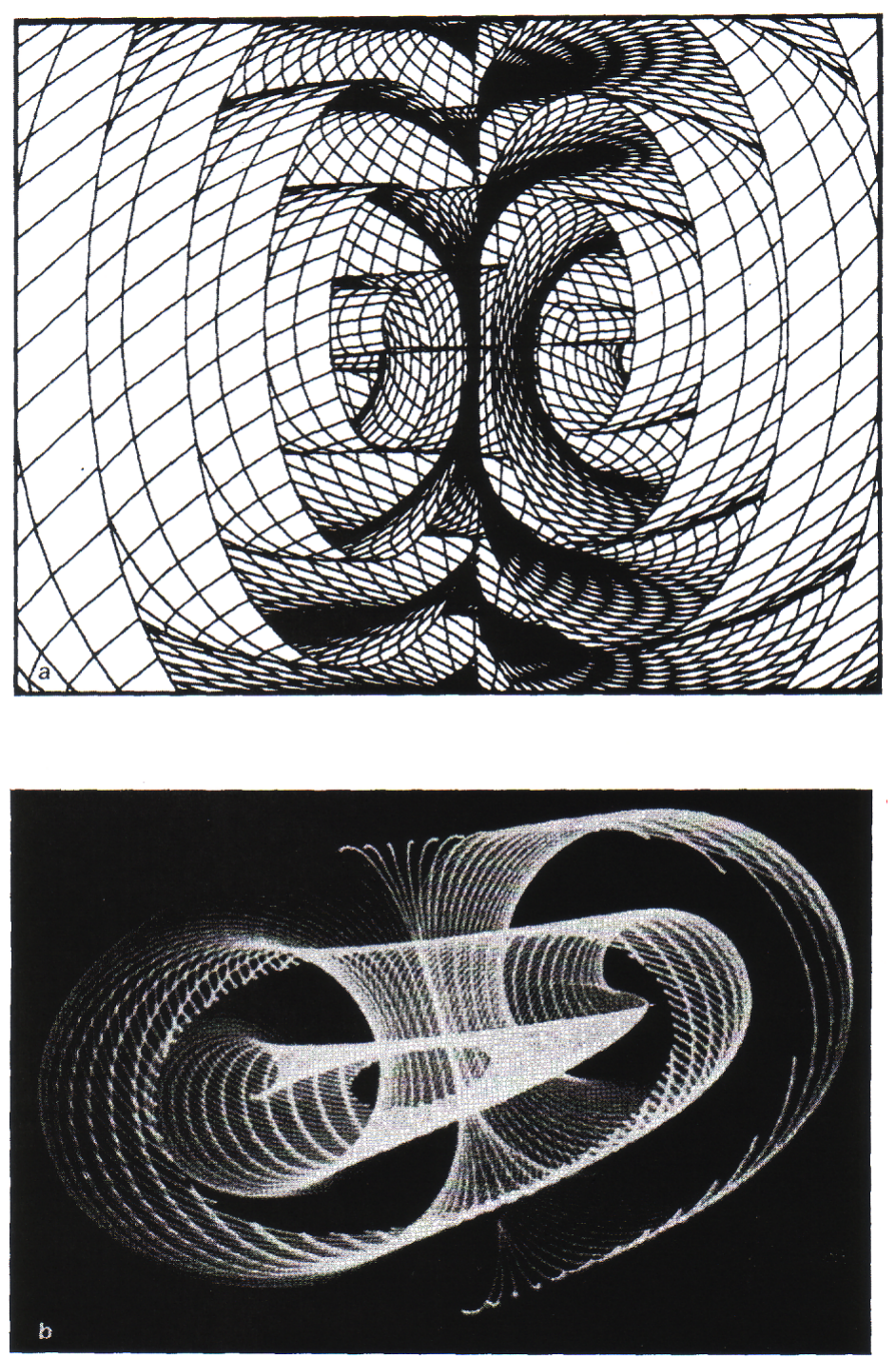

Fig. 2.27. Scroll waves are stacks of spirals (a). Twisted scroll wave (b). Courtesy of $[\mathrm{PaWi} 85]$. 


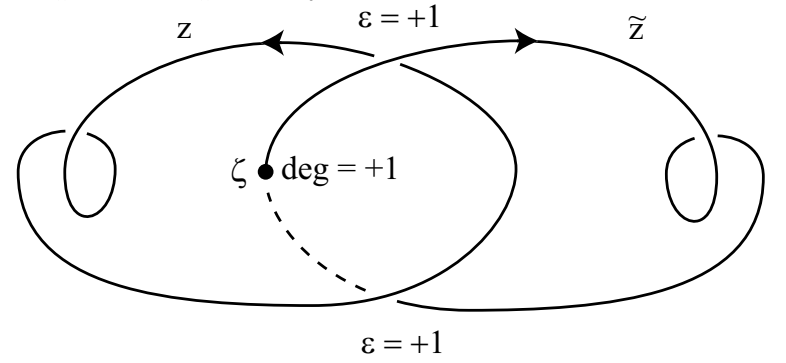

Fig. 2.28. Transverse crossings of planar projections of oriented curves, with associated signs $\varepsilon_{j}= \pm 1$. Note $\operatorname{lk}(z, \tilde{z})=+1$, and twist $(z)=\operatorname{twist}(\tilde{z})=-1$.

where the sum extends over all mutual crossings, but not over selfcrossings. Clearly, $\operatorname{lk}(\tilde{z}, z)=\operatorname{lk}(z, \tilde{z})$.

The linking number $\operatorname{lk}(\tilde{z}, z)$, and hence twist $(z)$, is independent of the choice of small enough $\delta>0$. The linking number does not depend on the choice of projection and is invariant under diffeotopies of $\mathbb{R}^{3}$; see for example [Ka87]. In particular, $\mathrm{lk} \neq 0$ implies $\tilde{z}, z$ cannot be unlinked, for example by a diffeotopy such that $\tilde{z}, z$ come to lie in the same plane.

The following proposition indicates how twisted scrolls have to be either knotted or else have to be linked to other twisted scrolls.

Proposition 2.6.1 Let $S \subset \Omega$ be an embedded surface, diffeomorphic to a closed disk, such that $\partial S$ is a closed filament curve $z(t, \cdot)$. Assume that $S$ is transverse to all other filament curves $z_{j}(t, \cdot) \subset u(t, \cdot)^{-1}(0)$ intersecting $S$. Let $\zeta_{k}$ enumerate these intersection points. Choose an orientation for $S$ such that the induced boundary orientation of $\partial S$ coincides with the orientation of the filament $z(t, \cdot)$. Then

$$
\operatorname{twist}(z)+\left.\sum_{x=\zeta_{k}} \operatorname{deg}_{l o c} u(t, \cdot)\right|_{S}=0
$$

For the proof, see [Du\&al85, §15.4].

Of course Proposition 2.6.1 holds only as long as $u=0$ is a regular value of the spatial solution profile $u(t, \cdot)$, for fixed time $t>0$. Neither linking nor knotting can change, in this situation. In the next section we will address the precise geometry of the failure of this assumption, which occurs at only discrete times $t>0$, for generic initial conditions $u_{0}$ at $t=0$.

\subsubsection{Generic Changes of Scroll Filament Topology}

How can two scroll wave filaments collide and "cross" each other as time $t$ evolves? In Figure 2.29 we show two linked closed scroll wave filaments $z_{1}(t, \cdot), z_{2}(t, \cdot)$, each parameterized over $\tau \in S^{1}$. In scenario (a), called "break through", the two circular filaments interpenetrate each other and restitute 
themselves as two unlinked circles $\tilde{z}_{1}, \tilde{z}_{2}$ after collision. The "crossover" scenario (b), in contrast, calls for a "kissing" of the two filaments $z_{1}(t, \cdot), z_{2}(t, \cdot)$ to occur, at time $t=t_{0}$, with a subsequent reconnection, such that a single closed filament $z$ is generated. Now, which scenario is right?

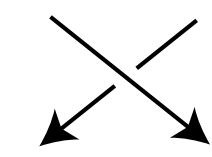

(a)

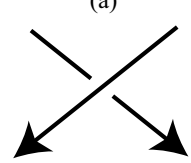

$\mathrm{t}<\mathrm{t}_{0}$

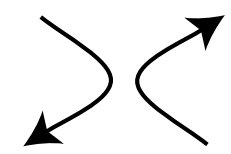

(b)

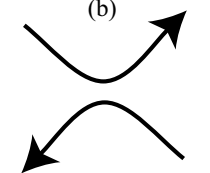

Fig. 2.29. Two scenarios for topology changes of linked scroll filaments: (a) break through; (b) crossover.

Note how filament orientation, as defined locally in (2.215), has to be respected by either scenario - and it is. Applying the scenario of Figure 2.29(a) to one of the mutual crossings in Figure 2.28 unlinks the two closed twisted filaments $z$ and $\tilde{z}$. Scenario $2.29(\mathrm{~b})$, in contrast, merges the two filaments to a single closed, untwisted filament, when applied between the top and bottom crossing.

Generic level sets To decide on the appropriate scenario we follow the approach in [FiMa00]. In Section 2.6.1 we have seen how the phenomenon of scroll wave filaments $x=z(t, \tau) \in \Omega$ mathematically corresponds to zeros $(t, x) \in(0, \infty) \times \Omega$ of solutions $u=u(t, x) \in \mathbb{R}^{2}$ of a reaction-diffusion system:

$$
u(t, x)=0 \text {. }
$$

If $u=u(t, x)$ was just "any" function, rather than a solution to a PDE, then singularity theory would provide ample information on the behavior of the zero set $\{(t, x) ; u(t, x)=0\}$. Indeed, generic functions $u$ only possess a finite number of singularities under our dimensional constraints. See for example [Ar\&al85, GoSc85, Ar93, Ar94b] for more details. We give specific examples below. However, $u=u(t, x)$ is not just "any" function: it is a solution $u=u\left(t, x ; u_{0}\right)$ of a reaction-diffusion system $(2.210)$ subject to boundary conditions and, more importantly, with prescribed initial conditions

$$
u(t=0, x)=u_{0}(x) .
$$

Theorem 2.6.2 [FiMa00] Let $k \geq 1$. Consider initial conditions $u_{0}$ in a Sobolev space $X$ which embeds into $C^{k}\left(\Omega, \mathbb{R}^{N}\right)$ and satisfies the (Neumann) boundary conditions. Then the solution $u(t, x)$ of (2.210) possesses only generic singularities, as determined by derivatives in $(t, x)$ up to order $k$, for generic initial conditions $u_{0} \in X$ and at any $t>0, x \in \Omega \subset \mathbb{R}^{m}$. 
Note that we use the word singularity in the sense of singularity theory here. We do not discuss "singularities" in the PDE-sense of blow-up of solutions or their derivatives. Nor are "singularities" understood as phase singularities, where some $\tilde{u}=u /|u| \in S^{1}$ remains undefined. Rather, a singularity is given by an algebraic variety $S$ in the jet space $J^{k}$ of Taylor polynomials up to order $k$ in the variables $(t, x)$. Consider the map

$$
\begin{aligned}
j^{k}:(0, \infty) \times \Omega & \rightarrow J^{k} \\
(t, x) & \mapsto j^{k} u(t, x)
\end{aligned}
$$

which maps $(t, x)$ to the $k$-jet $j^{k} u(t, x)$, the Taylor polynomial of order $k$ of the solution $u$, evaluated at $(t, x)$. Since $t>0$ and $x \in \Omega \subset \mathbb{R}^{m}$, together, are only $m+1$ independent variables, a generic function $u(t, x)$ will certainly miss a variety $S$ unless

$$
\operatorname{codim} S \leq m+1
$$

in $J^{k}$. The theorem states that, likewise, solutions $u=u\left(t, x ; u_{0}\right)$ of $(2.210)$ will miss such singularities $S$, at least for a subset $X^{\prime}$ of initial conditions $u_{0} \in X$ which is residual in the sense of Baire: $X^{\prime}$ contains at least a countable intersection of open dense subsets of $X$.

The proof of Theorem 2.6.2 is based on Thom transversality and backwards uniqueness for linear, nonautonomous parabolic systems. Essentially, Thom transversality reduces the problem to showing surjectivity of the linearization, with respect to $u_{0}$, of the $k$-jet of the solution $u$ at any fixed $(t, x)$. This finite-dimensional surjectivity property is then proved by a backwards uniqueness result which prevents solutions of linear equations to become zero in finite time. For a technically more complete account and slightly more general results we refer to [FiMa00]. For earlier results in a similar direction, of a purely local singularity theory flavor, see also [Dam97].

Sturm property, revisited We give some examples for our result, before returning to the problem of filament crossing. Since we are interested in zeros of solutions $u(t, x)$, the requirement $u=0 \in \mathbb{R}^{N}$ will always contribute $N$ to $\operatorname{codim} S$ in (2.224). Consider scalar equations, $N=1$, in one space dimension, $m=1$ first. To exclude $x$-profiles $x \mapsto u(t, x)$ with zeros of multiplicity three or higher, define $S$ by the requirement

$$
u=u_{x}=u_{x x}=0 .
$$

Since this implies codim $S=3>2=m+1$, the realization condition (2.224) is violated. Therefore (2.225) cannot occur, for generic initial conditions $u_{0} \in$ $X \hookrightarrow C^{2}$. Similarly, $u_{t} \neq 0$ at double zeros $u=u_{x}=0$, because

$$
u=u_{x}=u_{t}=0
$$

also implies $\operatorname{codim} S=3$. Therefore the only remaining local singularity basically takes the form 


$$
u(t, x)= \pm\left(t-t_{0}\right) \pm\left(x-x_{0}\right)^{2}
$$

see Figure 2.30. In Section 2.2.1 we have seen how $f=f\left(x, u, u_{x}\right)=0$ for $u=u_{x}=0$ implies that the two signs in (2.227) have to coincide. This Sturm property excludes case (b) of creation of a new pair of zeros, admitting only annihilation (a). Of course the Sturm property of Proposition 2.2.1, unlike our present genericity argument, is not limited to generic initial conditions $u_{0} \in X$.

(a)

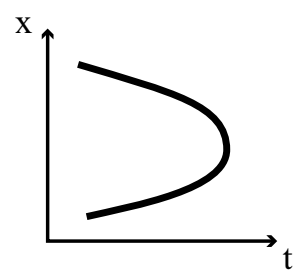

(b)

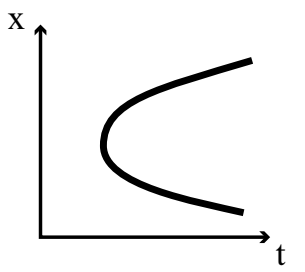

Fig. 2.30. Collision of zeros $u(t, x)=0$ for scalar equations: (a) annihilation, (b) creation.

Comparison principle and nodal domains For scalar equations, $N=1$, in arbitrary space dimension $m \geq 1$, Fig. 2.30 remains valid. Indeed the $m+1$ conditions

$$
u=0, \quad u_{x_{1}}=\cdots=u_{x_{m}}=0
$$

prevent further degeneracies to appear in generic singularities $S$, according to realization condition (2.224). In particular $u_{t} \neq 0$, and the Hessian matrix $u_{x x}$ has to be nondegenerate.

If the Hessian matrix $u_{x x}$ is strictly (positive or negative) definite, spherical bubbles of dimension $m-1$ are thus annihilated (a) or created (b). For $f=f(x, u, \nabla u)$ with $f(x, 0,0)=0$, however, creation (b) is again excluded by the partial differential equation which involves $\Delta u=$ trace $\left(u_{x x}\right)$. In the general, nongeneric case, this is due to the maximum (or comparison) principle; see Section 2.2.1, (2.12)-(2.14).

Suppose next that the Hessian $u_{x x}$ is indefinite. For simplicity we consider $m=\operatorname{dim} x=2$. In suitable local coordinates $x=\left(x_{1}, x_{2}\right)$, and normalizing to $x_{0}=0 \in \Omega$, we obtain the local form

$$
u(t, x)=\left(t-t_{0}\right)+x_{1}^{2}-x_{2}^{2}
$$

See Figure 2.31 for the curves describing the local zero set. This "Morse scenario" of course coincides with the behavior of level curves near a nondegenerate saddle point, with level values parameterized by time. Indeed $N=\operatorname{dim} u=1$, and $u_{t} \neq 0$ locally. The curves are oriented such that $u<0$ 


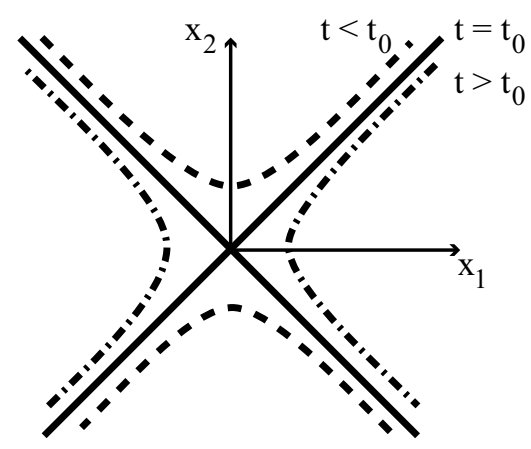

Fig. 2.31. Zero sets of $u(t, x)$ according to (2.229) as $t$ passes through $t_{0}$.

to the left of each curve. This orientation is the analogue, for $N=1$ and $m=2$, of the orientation of filament curves which was defined for $N=2$ and $m=3$ in Section 2.6.1. Note how the connectivity of the nodal domains $\{u(t, \cdot)>0\},\{u(t, \cdot)<0\}$ changes, as $t$ increases through $t_{0}$. Because the sign of $u_{t}=\Delta u=\operatorname{trace}\left(u_{x x}\right)$ at $\left(t_{0}, x_{0}\right)$ is indeterminate for indefinite Hessians $u_{x x}$, even when $f=f(x, 0,0)=0$, there is no analogue to the Sturm property in higher space dimensions $m \geq 2$ which could be based on counting nodal domains. See however [Uh76] for generic simplicity and sign change properties of eigenfunctions of the Laplacian in higher space dimensions.

Annihilation of spiral tips Finally we return to systems, $N=\operatorname{dim} u=$ 2, for example of the singularly perturbed FitzHugh-Nagumo type (2.210), (2.211). Consider $S=\left\{u=0, u_{x}=0\right\}$ which describes total degeneracy of the $N \times m$ Jacobian matrix $u_{x}$. Since codim $S=(N+1) m>m+1$, this singularity does not occur, for generic initial conditions $u_{0}$. LyapunovSchmidt reduction, based on rank $u_{x} \geq 1$, then reduces the discussion of all singularities to the case $\operatorname{dim} u=N-1=1$ and $\operatorname{dim} x=m-1$ which was already discussed above.

For example Figure 2.30(a), now corresponding to $N=2, m=2$, describes the coalescence and subsequent annihilation of a pair of spiral tips. Note how the invariance of Brouwer degree forces the two annihilating spiral tips to have opposite degree (2.213). For the two annihilating spirals this implies opposite rotation direction, in the Winfree paradigm. See also Figure 2.33 for a numerical simulation. It is noteworthy that the opposite phenomenon of persistent spiral pair creation, Figure 2.30(b), has not been observed, to our knowledge, neither numerically nor experimentally. This unobserved effect cannot be excluded by comparison or genericity principles.

Collisions of scroll wave filaments Let us next apply the same reduction to the case $N=2, m=3$ of scroll filament collision. By the above arguments, Lyapunov-Schmidt reduces this case to $N=1, m=2$. The annihilation 
for $t \nearrow t_{0}$ according to Figure 2.30(a), now of a circular filament $z(t, \cdot)$, has been observed. It corresponds to the collapse and disappearance of a scroll ring. Note that the collapsing scroll filament has to be non-twisted, due to Proposition 2.6.1. Again, the antagonistic case of scroll ring creation analogous to Figure 2.30(b) has mysteriously escaped both numerical and experimental documentation.

The only remaining singularity for $N=2, m=3$ alias $N=1, m=2$ is the case of indefinite (reduced) Hessian as depicted in Figure 2.31. Note how the entire dynamic process of approach (a), kiss (b), and reconnection (c) occurs in a single tangent plane. Moreover, Figure 2.31 gives the definitive answer to the opening question of this section: for generic initial conditions $u_{0} \in X \hookrightarrow C^{2}$, the topology changes of scroll wave filaments must occur by crossover collisions following the kissing scenario of Figure 2.29(b).

We summarize these results as follows.

Corollary 2.6.3 [FiMa00] For generic initial conditions $u_{0} \in X \hookrightarrow C^{2}$, only the following three collision scenarios of scroll wave filaments, as defined in (2.214) above, are possible for $t>0, x \in \Omega$ :

(i) annihilation of untwisted filaments, according to Figure 2.30(a);

(ii) creation of untwisted filaments, according to Figure 2.30(b);

(iii) crossover collision of filaments, according to Figures 2.29(b) and 2.31

We emphasize that this result applies only in the interior of the domain $\Omega$. We do not investigate, for example, the collision of filament points in the boundary $\Omega$.

As another corollary, we note that

$$
\sum_{j} \operatorname{twist}\left(z_{j}\right)+2 \sum_{j<k} \mathrm{lk}\left(z_{j}, z_{k}\right)=\text { const. }
$$

remains constant, for generic initial conditions $u_{0} \in X$, independently of the time evolution of the closed filaments $z_{j}=z_{j}(t, \cdot)$ and of possible changes in their number. Mutual collision, or self-collision, of closed filaments may change the total number of closed filaments but not the total twist and linking according to (2.230). It is again assumed here - similarly to degree theory - that the closed filaments $z_{j} \subset \Omega$ neither collide with the boundary $\partial \Omega$ nor with filaments $z$ which extend to the boundary.

For a proof we consider the accompanying filaments $\tilde{z}_{j} \subseteq u(t, \cdot)^{-1}(\delta, 0)$ which define the local phase along the scroll filament $z_{j}$. Then

$$
\sum_{j} \operatorname{twist}\left(z_{j}\right)+2 \sum_{j<k} \mathrm{lk}\left(z_{j}, z_{k}\right)=\sum_{j, k} \mathrm{lk}\left(z_{j}, \tilde{z}_{k}\right),
$$

for small enough $\delta<0$. None of the singularities (i)-(iii) of Corollary 2.6.3 changes the latter quantity. 
If all filaments are closed, so that $u \neq 0$ on $\partial \Omega \cong S^{2}$, then we note in passing that

$$
\sum_{j, k} \mathrm{lk}\left(z_{j}, \tilde{z}_{k}\right)=0
$$

by direct homotopy $u(t, \cdot) \rightarrow$ const. $\neq 0$.

For an example, we recall Figure 2.28. Another theoretical example well worth contemplating is the clover-shaped trefoil knot as shown in Figure 2.32. According to corollary (2.230) the initial torus knot must possess zero twist. One way to see this, directly, relies on the successive construction of Seifert surfaces $S$ : these are compact, orientable, embedded surfaces of minimal genus, with the link, or knot, as boundary. We may interpret $S$ as $u(t ; \cdot)^{-1}([0, \infty) \times\{0\})$. We start with an embedded disk spanning the circle, in the lower right of Figure 2.32. Crossover collisions change the topology of $S$, but in the present example $S$ will still qualify as a Seifert surface, both before and after collision. In the resulting Seifert surface of the original torus knot $z$, in the upper left of Figure 2.32, our interpretation produces $\tilde{z}=z(t ;)^{-1}((\delta, 0))$. Direct inspection then confirms zero twist of the torus knot, twist $(z)=\mathrm{lk}(z, \tilde{z})=0$, as predicted by (2.230). Similarly (2.230) shows that any single knot is untwisted. Indeed any single knot can be decomposed into unlinked planar circles, under a suitable sequence of crossover collisions. Any twisted knot, in contrast, must be linked to another filament, again by (2.230). If two knots are mutually linked, but isolated otherwise, then at least one of the knots must be twisted. These remarks generalize our previous conclusions from proposition 6.1. For a numerical example see Section 2.6.3.
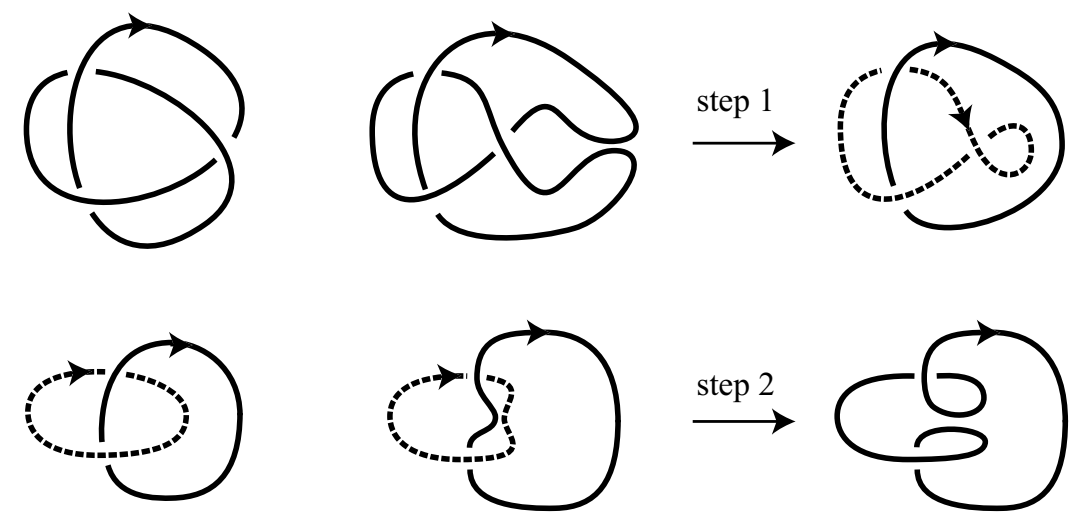

Fig. 2.32. Schematic two-step disintegration of a torus knot filament via linked filaments. 


\subsubsection{Numerical Simulations}

In the previous two sections we have presented mathematically rigorous results concerning degrees, orientations, twists, linking, and collisions of zeros of solutions $u=u(t, x)$ of reaction-diffusion systems. The terminology of spiral tip, scroll waves, scroll wave filaments, etc. was largely motivated by experimental observations and numerical simulations. We present some such simulations in the present section. All results presented here are based on [FiMa00]. The online version contains downloadable animations, for the entertainment of our readers! As a model equation we consider the FitzHughNagumo system (2.210), (2.211). For parameters we choose

$$
\begin{aligned}
a= & 0.8 ; \\
b= & 0.01+A \cos \omega t \quad \text { with } \\
& A=0 \text { or } 0.01 ; \omega=3.21 ; \\
D= & \left(\begin{array}{cc}
1 & 0 \\
0 & 0.5
\end{array}\right) ; \\
\varepsilon= & 0.02 ; \\
\Omega= & {[-15,15]^{3} ; }
\end{aligned}
$$

unless stated otherwise. To define tip and filament positions, we use the shifted reference values $u_{1}=u_{2}=0.5$ rather than $u=0$. The choices of $A$ correspond to autonomous versus slightly periodically forced simulations.

For integration we use Barkley's code [Do\&al97] with equidistant $x$ discretization by $125^{3}$ points and a time step $\Delta t=0.0172 \cdots$. Filament positions are determined from the discrete data by a simplex method in the spirit of [AlGe90], compatible with the above degree and orientation approach. To visualize the local phase, as well as accompanying twist curves $\tilde{z}$, we compute and plot a piecewise linear approximation to the surface in $\Omega$ given by the preimage under $u(t, \cdot)$ of the band

$$
u_{2}=0.5 \leq u_{1} \leq 0.5+\delta .
$$

Following the Winfree paradigm, we call this surface with boundaries $z$ and $\tilde{z}$ the isochrone band.

The choice of initial conditions which produce filament collisions can be a delicate issue. For planar simulations, $m=\operatorname{dim} x=2$, it is relatively easy to prescribe "vector fields" $u_{0}=u_{0}(x)$ with zeros of prescribed degree. The reaction-diffusion system then quickly produces spirals with tips, near these zeros, and with rotation directions according to local Brouwer degree. Collisions can then be produced, with a little bit of forcing. For three-dimensional simulations, [HeWi91] has described a method which can produce linked circular filaments and torus knots. This method is based on algebraic topology: the initial filament configurations in $x \in \Omega$ are realized by zeros $z$ of appropriate complex polynomials $u(x)=p(z) \in \mathbb{C} \cong \mathbb{R}^{2}$, in two complex variables $z=\left(z_{1}, z_{2}\right)$. By inverse stereographic projection $\iota$, we have $x \in \Omega \subset \mathbb{R}^{3} \stackrel{\iota}{\hookrightarrow} S^{3} \subset \mathbb{C}^{2}$ corresponding to the argument $z$ of $p(z)$. 
We now describe the individual runs. The planar simulation of Figure 2.33 shows color coded solution profiles $u_{1}(t, \cdot)$ corresponding to two meandering spirals which approach each other and annihilate. The domain $\Omega=[-15,15]^{2}$ was discretized by $125^{2}$ points. Slightly differently from Section 2.5.2, the meandering motion is produced by an external time-periodic, spatially homogeneous forcing $A=0.01$. The frequency $\omega=3.21$ and the phase were tuned to near drift resonance, so that the two spirals are moving towards each other, initially. Rather than mutual repulsion, annihilation occurs. Note the meandering, near drifting behavior of the spiral tips $u_{1}=u_{2}=0.5$, traced in white.

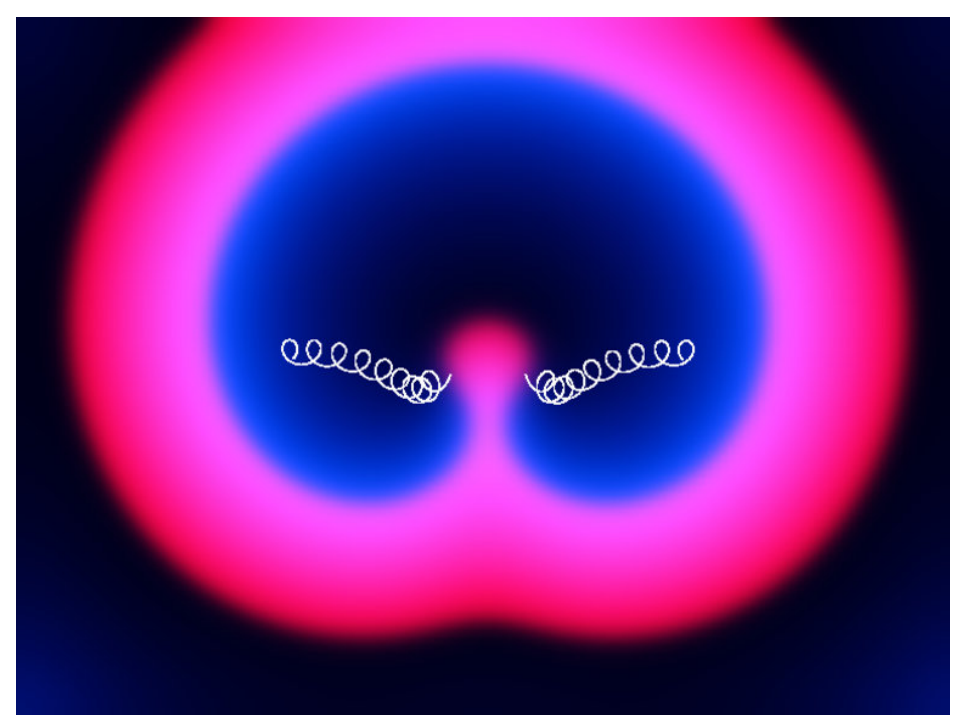

Fig. 2.33. [FiMa00, Fig. 7] Meandering interaction and collision of a pair of planar spiral waves. See Plate ?? in the Appendix for a version of this figure in colour.

The annihilation of a three-dimensional untwisted scroll ring in Figure 2.34 is an autonomous simulation, $A=0$. Note the analogy to the planar simulation of spiral pair annihilation, by viewing adynsys/dynsysfig/ planar vertical slice through the toroidal scroll ring. The transparent full isochrone surfaces in $\Omega=[-15,15]^{3}$ given by $u_{2}=0.5 \leq u_{1}$ and shown in this example would obscure the view on the scroll filaments $u_{2}=0.5=u_{1}$ in more complicated simulations. Therefore they are replaced by the truncated isochrone bands $u_{2}=0.5 \leq u_{1} \leq 0.5+\delta$, as was explained above.

Crossover collision is documented in Figure 2.35. For finer resolution we choose a smaller domain $\Omega=[-10,10]^{3}$, again with $125^{3}$ discretization points. The appropriately adjusted time step is $\Delta t=0.00765$. We choose two straight line horizontal filaments, orthogonal to each other and at a ver- 

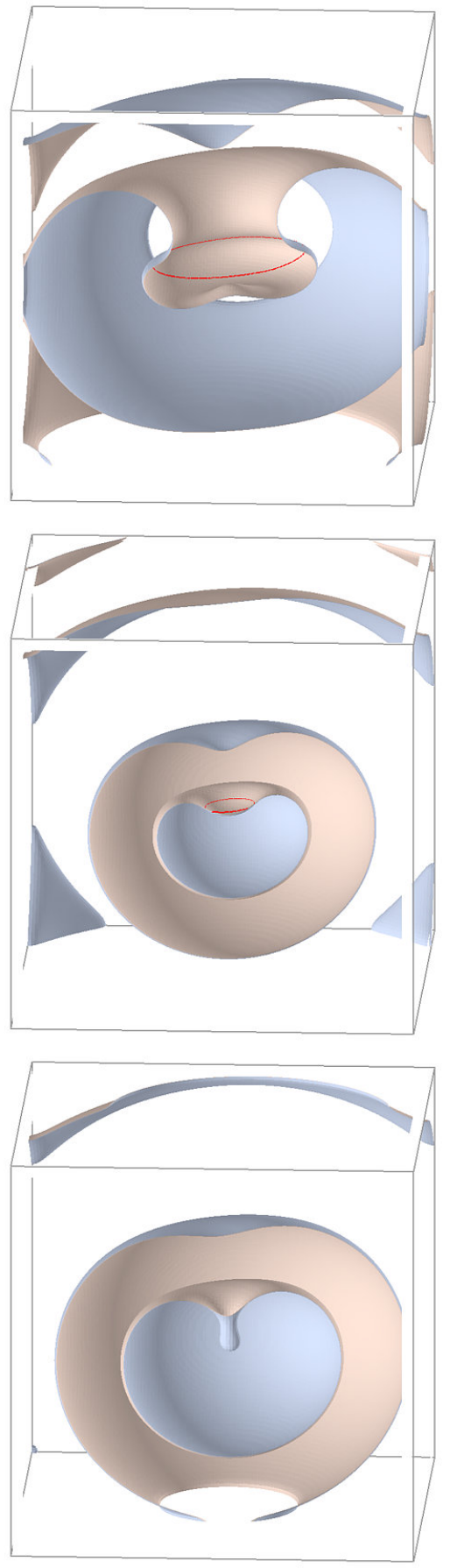

Fig. 2.34. [FiMa00, Fig. 8] Scroll ring annihilation of an untwisted filament. Surfaces $u_{2}=0 \leq u_{1}$ shown. See Plate ?? in the Appendix for a version of this figure in colour. 

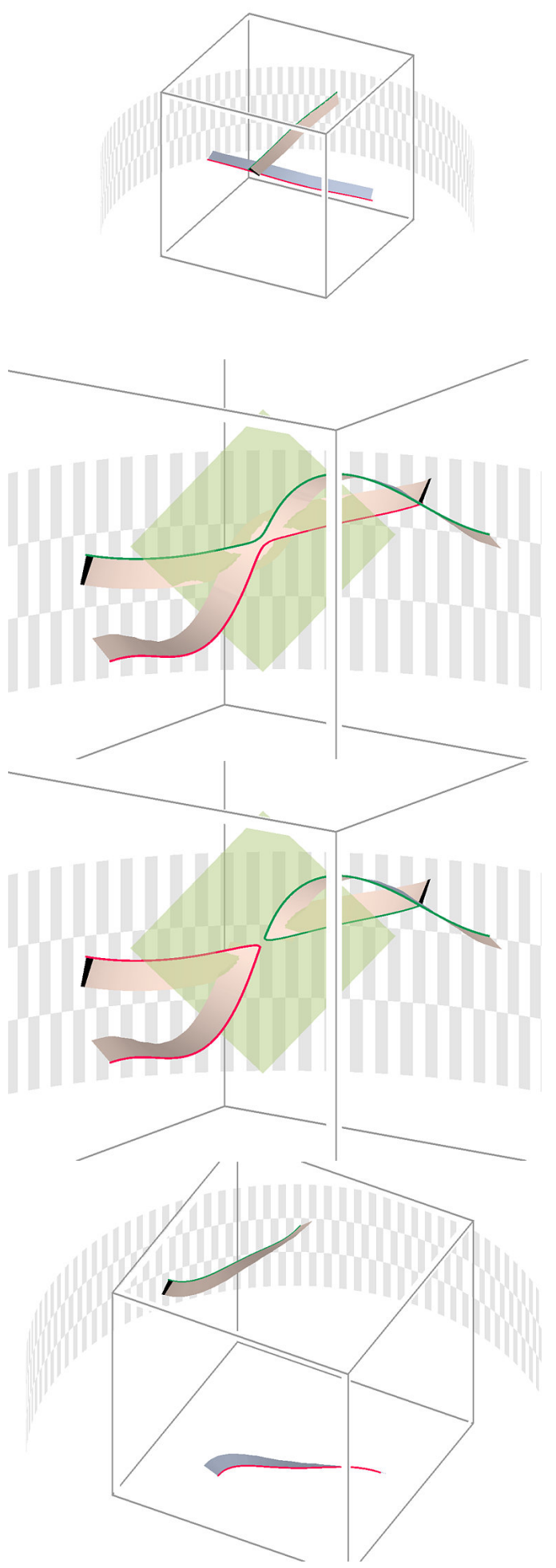

Fig. 2.35. [FiMa00, Fig. 9] Crossover collision of scroll waves including accompanying isochrone bands $u_{2}=0 \leq u_{1} \leq \delta$. See Plate ?? in the Appendix for a version of this figure in colour. 

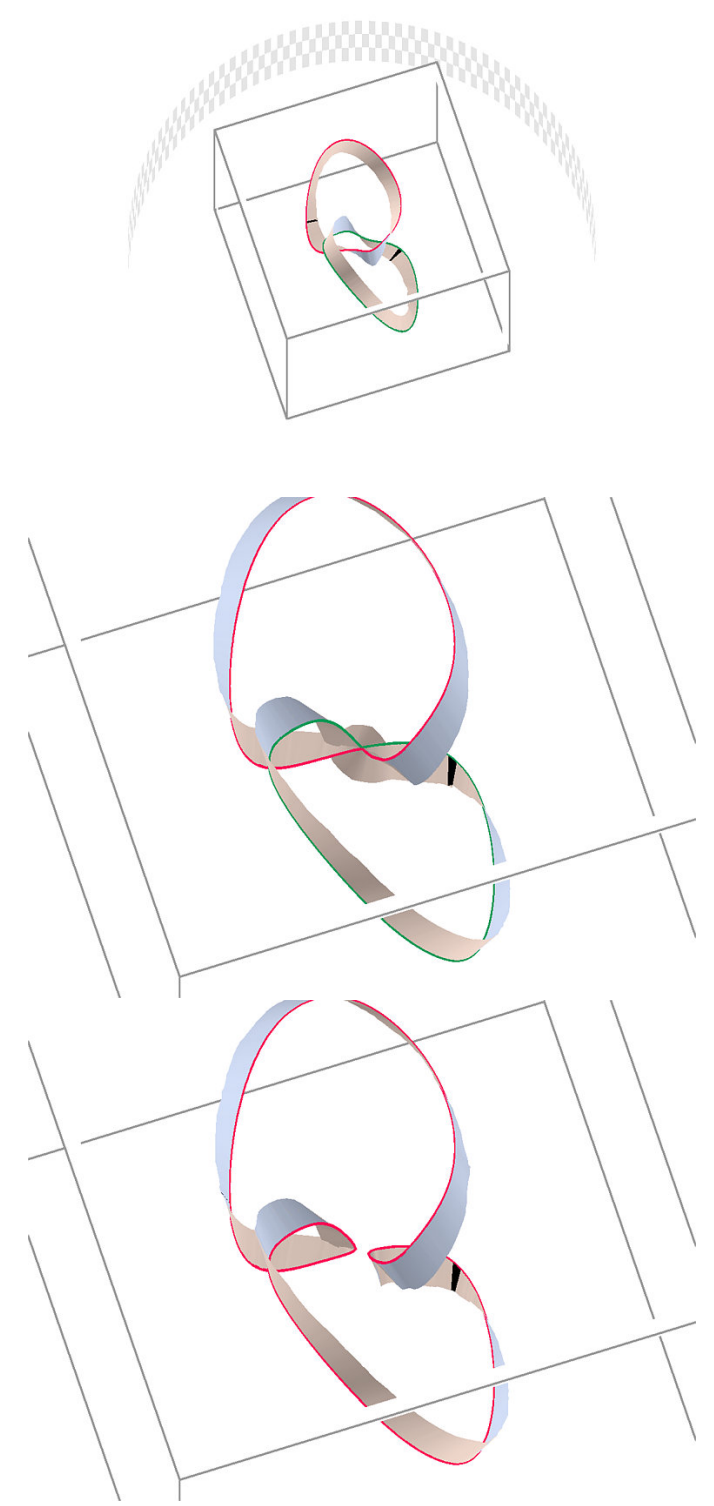

Fig. 2.36. [FiMa00, Fig. 11] Crossover collision of two linked, twisted, circular filaments. See Plate ?? in the Appendix for a version of this figure in colour. 

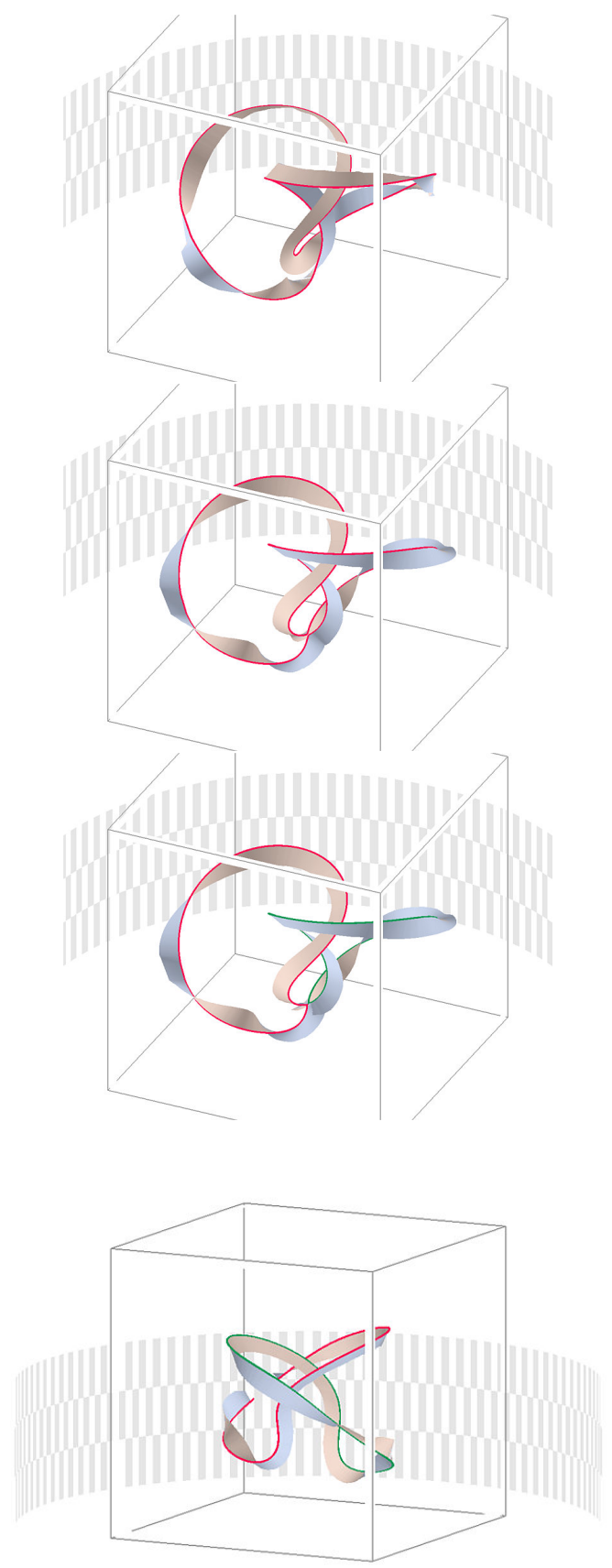

Fig. 2.37. [FiMa00, Fig. 13] Decomposition of the trefoil knot into two linked, twisted circular filaments. See Plate ?? in the Appendix for a version of this figure in colour. 
tical distance. By a spatially homogeneous, time periodic forcing $A=0.01$, as before, we can make the top filament drift downwards and the bottom filament drift upwards. Because the break through scenario of Figure 2.29(a) is forbidden, generically, the filaments have to first locate a tangent plane for kissing. Indeed, the crossover collision then occurs between $t=35$ and $t=40$.

Based on the polynomial $z(z)=z_{1}^{2}-z_{2}^{2}$, an unlinking collision of two linked, twisted, circular scroll filaments is shown in Figure 2.36. The example is autonomous, $A=0$, with data as in (2.233). Again a common tangent plane is located. Kissing and reconnection according to the abstract scenario of Figures 2.28, 2.29(b), and 2.31 occurs between $t=4.8$ and $t=4.9$.

Our final simulation, the decomposition of a trefoil knot into two linked, twisted, circular scroll filaments, runs on a spatially coarser $125^{3}$ grid on $\Omega=$ $[-25,25]^{3}$. The construction of the initial condition is based on the polynomial $p(z)=z_{1}^{2}-z_{2}^{3}$. The autonomous run, $A=0$, follows the theoretical scenario of Figures 2.29(b) and 2.32, fully compliant with our abstract theory.

Our theoretical results apply to any fixed value $u=c$ and the associated level curves of the spatial profiles. It is the prominence of the particular values associated to spiral tips, however, becoming visible only through experimental work and numerical simulation along the Winfree paradigm, which has motivated our mathematical analysis.

\section{References}

[AbRo67] R. Abraham and J. Robbin. Transversal Mappings and Flows. Benjamin Inc., Amsterdam, 1967.

[Al79] N. Alikakos. An application of the invariance principle to reaction diffusion equations. J. Diff. Eqns. 33 (1979), 201-225.

[Al\&al89] N. Alikakos, P.W. Bates, and G. Fusco. Slow motion for the CahnHilliard equation in one space dimension. Preprint (1989).

[AlGe90] E.L. Allgower and K. Georg. Numerical Continuation Methods. An Introduction. Springer-Verlag, Berlin, 1990.

[An86] S. Angenent. The Morse-Smale property for a semi-linear parabolic equation. J. Diff. Eqns. 62 (1986), 427-442.

[An88] S. Angenent. The zero set of a solution of a parabolic equation. Crelle J. reine angew. Math., 390 (1988), 79-96.

[An90] S. Angenent. Parabolic equations for curves on surfaces. I: curves with $p$ integrable curvature. Ann. Math. 132 (1990), 451-483.

[An91] S. Angenent. Parabolic equations for curves on surfaces. II: Intersections, blow-up and generalized solutions. Ann. Math., 133 (1991), 171-215.

[An93] S. Angenent. A variational interpretation of Melnikov's function and exponentially small separatrix splitting. Lond. Math. Soc. Lect. Note Ser., 192 (1993), 5-35.

[AnFi88] S. Angenent and B. Fiedler. The dynamics of rotating waves in scalar reaction diffusion equations. Trans. Amer. Math. Soc., 307 (1988), 545-568. 
[An\&al87] S. Angenent, J. Mallet-Paret, and L.A. Peletier. Stable transition layers in a semilinear boundary value problem. J. Diff. Eqns. 67 (1987), 212-242.

[An91] D.V. Anosov. Dynamical Systems with Hyperbolic Behaviour. Enc. Math. Sc. 66, Dynamical Systems IX. Springer-Verlag, New York, 1991.

[AnAr88] D. V. Anosov and V. I. Arnol'd. Ordinary differential equations and smooth dynamical systems. Enc. Math. Sc. 1, Dynamical Systems I. SpringerVerlag, Berlin, 1988.

[Ar\&al92] I. S. Aranson, L. Aranson, L. Kramer, and A. Weber. Stability limits of spirals and travelling waves in nonequilibrium media. Phys. Rev. A 46 (1992), 2992-2995.

[Ar\&al94] I.S. Aranson, L. Kramer, and A. Weber. Core instability and spatiotemporal intermittency of spiral waves in oscillatory media. Phys. Rev. Lett. 72, 2316 (1994).

[Ar92] V.I. Arnol'd. Theory of Bifurcations and Catastrophes. Enc. Math. Sc. 5, Dynamical Systems V. Springer-Verlag, Berlin, 1992.

[Ar93] V.I. Arnol'd. Singularity Theory I. Enc. Math. Sc. 6, Dynamical Systems VI. Springer-Verlag, New York, 1993.

[Ar94a] V.I. Arnol'd. Bifurcation Theory and Catastrophe Theory. Enc. Math. Sc. 5, Dynamical Systems V. Springer-Verlag, New York, 1994.

[Ar94b] V.I. Arnol'd. Singularity theory II, Applications. Enc. Math. Sc. 8, Dynamical Systems VIII. Springer-Verlag, New York, 1993.

[Ar\&al85] V.I. Arnol'd, S.M. Gusejn-Zade, and A.N. Varchenko. Singularities of Differentiable Maps. Volume I: The Classification of Critical points, Caustics and Wave Fronts. Birkhäuser, Boston, 1985.

[Ar\&al88] V.I. Arnol'd, V.V. Kozlov, and A.I. Neishtadt. Mathematical Aspects of Classical and Celestial Mechanics. Enc. Math. Sc. 3, Dynamical Systems III. Springer-Verlag, New York, 1988.

[ArNo90] V.I. Arnol'd and S.P. Novikov. Symplectic Geometry and its Applications. Enc. Math. Sc. 4, Dynamical Systems IV. Springer-Verlag, New York, 1990.

[ArNo94] V.I. Arnol'd and S.P. Novikov. Integrable Systems. Nonholonomic Dynamical Systems. Enc. Math. Sc. 16, Dynamical Systems VII. Springer-Verlag, New York, 1994.

[ArVi89] V.I. Arnol'd and M.I. Vishik et al. Some solved and unsolved problems in the theory of differential equations and mathematical physics. Russian Math. Surveys, 44 (1989), 157-171.

[AM97] P. Ashwin and I. Melbourne. Noncompact drift for relative equilibria and relative periodic orbits. Nonlinearity, 10 (1997), 595-616.

[As\&al99] P. Ashwin, I. Melbourne, and M. Nicol. Drift bifurcations of relative equilibria and transitions of spiral waves. Nonlinearity 12 (1999), 741-755.

[As\&al01] P. Ashwin, I. Melbourne, and M. Nicol. Hypermeander of spirals: local bifurcations and statistical properties. Phys. D 156 (2001), 364-382.

[BaVi92] A.V. Babin and M.I. Vishik. Attractors of Evolution Equations. North Holland, Amsterdam, 1992.

[BäEi93] M. Bär and M. Eiswirth. Turbulence due to spiral breakup in a continuous excitable medium. Phys. Rev. E 48 (1993), 1635-1637.

[BäOr99] M. Bär and M. Or-Guil. Alternative scenarios of spiral breakup in a reaction-diffusion model with excitable and oscillatory dynamics. Phys. Rev. Lett. 82 (1999), 1160-1163.

[Ba92] D. Barkley. Linear stability analysis of rotating spiral waves in excitable media. Phys. Rev. Lett. 68 (1992), 2090-2093. 
[Ba93] D. Barkley. Euclidean symmetry and the dynamics of rotating spiral waves. Phys. Rev. Lett. 72 (1994), 164-167.

[Bar95] D. Barkley. Spiral meandering. In R. Kapral and K. Showalter (eds.), Chemical Waves and Patterns, p.163-190, Kluwer, 1995.

[Ba\&al93] G. Barles, H.M. Soner, and P.E. Souganidis. Front propagation and phase field theory. SIAM J. Contr. Optim. 31 (1993), 439-469.

[Be\&a197] A. Belmonte, J.-M. Flesselles, and Q. Ouyang. Experimental Survey of Spiral Dynamics in the Belousov-Zhabotinsky Reaction. J. Physique II 7 (1997), 1425-1468.

[BeNi90] H. Berestycki and L. Nirenberg. Some qualitative properties of solutions of semilinear elliptic equations in cylindrical domains. Coll. Analysis, et cetera, 115-164, Academic Press Boston, 1990.

[BePh96] I. Berkes and W. Philipp. Trigonometric series and uniform distribution mod 1. Stud. Sci. Math. Hung. 31 (1996), 15-25.

[Be87] W.J. Beyn. The effect of discretization on homoclinic orbits. In Bifurcation: Analysis, Algorithms, Applications 1-8, T. Küpper et al., (eds.). Birkhäuser Verlag, Basel, 1987.

[Be90] W.-J. Beyn. The numerical computation of connecting orbits in dynamical systems. IMA Z. Numer. Anal, 9 (1990), 379-405.

[Bi\&al96] V. A. Biktashev, A. V. Holden, and E. V. Nikolaev. Spiral wave meander and symmetry of the plane. Preprint, University of Leeds, 1996.

[BiRo62] G. Birkhoff and G.-C. Rota. Ordinary differential equations. Ginn and Company, Boston, 1962.

[Bl\&al00] P. Blancheau, J. Boissonade, and P. De Kepper. Theoretical and experimental studies of bistability in the chloride-dioxide-iodide reaction. Physica $D$ 147 (2000), 283-299.

[Bo81a] R. Bogdanov. Bifurcation of the limit cycle of a family of plane vector fields. Sel. Mat. Sov. 1 (1981), 373-387.

[Bo81b] R. Bogdanov. Versal deformations of a singularity of a vector field on the plane in the case of zero eigenvalues. Sel. Mat. Sov., 1 (1981), 389-421.

[BrEn93] M. Braune and H. Engel. Compound rotation of spiral waves in a lightsensitive Belousov-Zhabotinsky medium. Chem. Phys. Lett. 204 (1993), 257264.

[Br64] R. J. Briggs. Electron-Steam Interaction With Plasmas. MIT press, Cambridge, 1964.

[BrtD85] T. Bröcker and T. tom Dieck. Representations of Compact Lie Groups. Springer-Verlag, Berlin, 1985.

[Br\&al01] H.W. Broer, B. Krauskopf, and G. Vegter. Global Analysis of Dynamical Systems. IOP Publishing, Bristol, 2001.

[BrTa02] H. Broer and T. Takens (eds.). Handbook of Dynamical Systems 3. Elsevier, Amsterdam, in preparation 2002.

[Br90] P. Brunovský. The attracor of the scalar reaction diffusion equation is a smooth graph. J. Dynamics and Differential Equations, 2 (1990), 293-323.

[BrCh84] P. Brunovský and S-N Chow. Generic properties of stationary state solutions of reaction-diffusion equations. J. Diff. Eqns. 53 (1984), 1-23.

[BrFi86] P. Brunovský and B. Fiedler. Numbers of zeros on invariant manifolds in reaction-diffusion equations. Nonlin. Analysis, TMA, 10 (1986), 179-194.

[BrFi88] P. Brunovský and B. Fiedler. Connecting orbits in scalar reaction diffusion equations. Dynamics Reported 1 (1988), 57-89. 
[BrFi89] P. Brunovský and B. Fiedler. Connecting orbits in scalar reaction diffusion equations II: The complete solution. J. Diff. Eqns. 81 (1989), 106-135.

[Br\&al92] P. Brunovský, P. Poláčik, and B. Sandstede. Convergence in general parabolic equations in one space dimension. Nonl. Analysis TMA 18 (1992), 209-215.

[Ca\&al93] A. Calsina, X. Mora and J. Solà-Morales. The dynamical approach to elliptic problems in cylindrical domains and a study of their parabolic singular limit. J. Diff. Eqns. 102 (1993), 244-304.

[CaPe90] J. Carr and R. Pego.Invariant manifolds for metastable patterns in $u \bar{s} t=$ $\epsilon^{2} u \bar{s} x x-f(u)$. Proc. Roy. Soc. Edinburgh A 116 (1990), 133-160.

[Ca\&al90] V. Castets, E. Dulos, J. Boissonade, and P. De Kepper. Experimental evidence of a sustained standing Turing-type nonequilibrium chemical pattern. Phys. Rev. Lett. 64 (1990), 2953-2956.

[ChIn74] N. Chafee and E. Infante. A bifurcation problem for a nonlinear parabolic equation. J. Applicable Analysis 4 (1974). 17-37.

[Ch98] X.-Y. Chen. A strong unique continuation theorem for parabolic equations. Math. Ann. 311 (1998), 603-630.

[ChVi02] V.V. Chepyzhov and M.I. Vishik. Attractors for Equations of Mathematical Physics. Colloq. AMS, Providence, 2002.

[ChLa00] P. Chossat and R. Lauterbach. Methods in Equivariant Bifurcations and Dynamical Systems. World Scientific, Singapore, 2000.

[ChHa82] S.-N. Chow and J. K. Hale. Methods of Bifurcation Theory. SpringerVerlag, New York, 1982.

[CoEc00] P. Collet and J.-P. Eckmann. Proof of the marginal stability bound for the Swift-Hohenberg equation and related equations. Preprint, 2000.

[Co68] W.A. Coppel. Dichotomies and reducibility II. J. Diff. Eqns. 4 (1968), 386398.

[Co78] W.A. Coppel. Dichotomies in Stability Theory. Lect. Notes Math. 629, Springer, Berlin, 1978.

[CrHo93] M.C. Cross and P.C. Hohenberg. Pattern formation outside equilibrium. Rev. Modern Phys. 65 (1993), 851-1112.

[Dam97] J. Damon. Generic properties of solutions to partial differential equations. Arch. Rat. Mech. Analysis, 140 (1997), 353-403.

[DaPo02] E.N. Dancer and P. Poláčik. Realization of vector fields and dynamics of spatially homogeneous parabolic equations. Mem. AMS, Providence, 2002, to appear.

[Da\&al96] G. Dangelmayr, B. Fiedler, K. Kirchgässner, and A. Mielke. Dynamics of Nonlinear Waves in Dissipative Systems: Reduction, Bifurcation and Stability. Pitman 352, Boston, 1996.

[De\&al95] M. Dellnitz, M. Golubitsky, A. Hohmann,and I. Stewart. Spirals in scalar reaction-diffusion equations. Internat. J. Bifur. Chaos Appl. Sci. Engrg. 5 (1995), 1487-1501.

[De85] K. Deimling. Nonlinear Functional Analysis. Springer-Verlag, Berlin, 1985.

[dK\&al94] P. de Kepper, J.-J. Perraud, B. Rudovics,and E. Dulos. Experimental study of stationary Turing patterns and their interaction with traveling waves in a chemical system. Int. J. Bifurcation Chaos Appl. Sci. Eng. 4 (1994), 12151231.

[Di\&al95] O. Diekmann, S.A. v. Gils, S.M. Verduyn Lunel, and H.-O. Walther. Delay Equations. Functional-, Complex-, and Nonlinear Analysis. Springer-Verlag, New York, 1995. 
[DoFr89] E. J. Doedel and M. J. Friedman. Numerical computation of heteroclinic orbits. J. Comp. Appl. Math.26 (1989), 155-170.

[DoFr91] E. J. Doedel and M. J. Friedman. Numerical computation and continuation of invariant manifolds connecting fixed points. SIAM J. Numer. Anal. 28 (1991), 789-808.

[Do\&al97] M. Dowle, M. Mantel, and D. Barkley. Fast simulations of waves in three-dimensional excitable media. Int. J. Bifur. Chaos, 7 (1997), 2529-2546.

[Du\&al85] B.A. Dubrovin, A.T. Fomenko, and S.P. Novikov. Modern Geometry Methods and Applications. Part 2: The Geometry and Topology of Manifolds. Springer-Verlag, New York, 1985.

[EiYa98] S.-I. Ei and E. Yanagida. Slow dynamics of interfaces in the allen-cahn equation on a strip-like domain. SIAM J. Math. Anal., 29 (1998), 555-595.

[El\&al87] C. Elphick, E. Tirapegui, M.E. Brachet, P. Coullet, and G. Iooss. A simple global characerization for normal forms of singular vector fields. Physica 29D (1987), 95-127.

[Fe71] N. Fenichel. Persistence and smoothness of invariant manifolds for flows. Indiana Univ. Math. J. 21 (1971), 193-226.

[Fe74] N. Fenichel. Asymptotic stability with rate conditions. Indiana Univ. Math. J. 23 (1974), 1109-1137.

[Fe77] N. Fenichel. Asymptotic stability with rate conditions, II. Indiana Univ. Math. J. 26 (1977), 81-93.

[Fe79] N. Fenichel. Geometric singular perturbation theory for ordinary differential equations. J. Diff. Eqns., 31 (1979), 53-98.

[Fi88] B. Fiedler. Global Bifurcation of Periodic Solutions with Symmetry. SpringerVerlag, Berlin, 1988.

[Fi89] B. Fiedler. Discrete Ljapunov functionals and $\omega$-limit sets. Math. Mod. Num. Analysis, 23 (1989), 415-431.

[Fi94] B. Fiedler. Global attractors of one-dimensional parabolic equations: sixteen examples. Tatra Mountains Math. Publ., 4 (1994), 67-92.

[Fi96] B. Fiedler. Do global attractors depend on boundary conditions? Doc. Math. 1 (1996), 215-228.

[Fi02] B. Fiedler (ed.) Handbook of Dynamical Systems 2, Elsevier, Amsterdam. In press.

[FiGe98] B. Fiedler and T. Gedeon. A class of convergent neural network dynamics. Physica D, 111 (1998), 288-294,.

[FiGe99] B. Fiedler and T. Gedeon. A Lyapunov function for tridiagonal competitive-cooperative systems. SIAM J. Math Analysis 30 (1999), 469-478.

[Fi\&al00] B. Fiedler, K. Gröger, and J. Sprekels (eds.). Equadiff 99. International Conference on Differential Equations, Berlin 1999. Vol.1,2. World Scientific, Singapore, 2000.

[FiMP89a] B. Fiedler and J. Mallet-Paret. Connections between Morse sets for delay-differential equations. J. reine angew. Math., 397:23-41, (1989).

[FiMP89b] B. Fiedler and J. Mallet-Paret. A Poincaré-Bendixson theorem for scalar reaction diffusion equations. Arch. Rat. Mech. Analysis 107 (1989), 325345.

[FiMa00] B. Fiedler and R.-M. Mantel. Crossover collision of core filaments in three-dimensional scroll wave patterns. Doc. Math. 5 (2000), 695-731.

[FiPo90] B. Fiedler and P. Poláčik. Complicated dynamics of scalar reaction diffusion equations with a nonlocal term. Proc. Royal Soc. Edinburgh 115A (1990), 167-192. 
[FiRo96] B. Fiedler and C. Rocha. Heteroclinic orbits of semilinear parabolic equations. J. Diff. Eq. 125 (1996), 239-281.

[FiRo99] B. Fiedler and C. Rocha. Realization of meander permutations by boundary value problems. J. Diff. Eqns. 156 (1999), 282-308.

[FiRo00] B. Fiedler and C. Rocha. Orbit equivalence of global attractors of semilinear parabolic differential equations. Trans. Amer. Math. Soc., 352 (2000), $257-284$.

[Fi\&al02a] B. Fiedler, C. Rocha, D. Salazar, and J. Sol-Morales. A note on the dynamics of piecewise-autonomous bistable parabolic equations. Comm. Fields Inst. (2002), in press.

[Fi\&al02b] B. Fiedler, C. Rocha, and M. Wolfrum. Heteroclinic connections of $S^{1}$ equivariant parabolic equations on the circle. In preparation, 2002.

[Fi\&al96] B. Fiedler, B. Sandstede, A. Scheel, and C. Wulff. Bifurcation from relative equilibria of noncompact group actions: skew products, meanders and drifts. Doc. Math. J. DMV 1 (1996), 479-505. See also http://www.mathematik.uni-bielefeld.de/documenta/vol-01/20.ps.gz

[Fi\&al98] B. Fiedler, A. Scheel, and M. Vishik. Large patterns of elliptic systems in infinite cylinders. J. Math. Pures Appl. 77 (1998), 879-907.

[FiTu98] B. Fiedler and D. Turaev. Normal forms, resonances, and meandering tip motions near relative equilibria of Euclidean group actions. Arch. Rat. Mech. Anal. 145 (1998), 129-159.

[FiVi01a] B. Fiedler and M. Vishik. Quantitative homogenization of analytic semigroups and reaction diffusion equations with diophantine spatial frequencies. Adv. in Diff. Eqns. 6 (2001), 1377-1408.

[FiVi01b] B. Fiedler and M. Vishik. Quantitative homogenization of global attractors for reaction-diffusion systems with rapidly oscillating terms. Preprint, 2001.

[Fi88] P.C. Fife. Dynamics of internal layers and diffusive interfaces, CBMS-NSF Reg. Conf. Ser. Appl. Math. 53, 1988.

[Fi84] G. Fischer. Zentrumsmannigfaltigkeiten bei elliptischen Differentialgleichungen. Math. Nachr. 115 (1984), 137-157.

[Fr64] A. Friedman. Partial Differential Equations of Parabolic Type. Prentice Hall, Englewood Cliffs, New Jersey, 1964.

[FuHa89] G. Fusco and J.K. Hale. Slow-motion manifolds, dormant instability, and singular perturbations. J. Dyn. Diff. Eqns. 1 (1989), 75-94.

[FuOl88] G. Fusco and W.M. Oliva. Jacobi matrices and transversality. Proc. Royal Soc. Edinburgh A 109 (1988), 231-243.

[FuRo91] G. Fusco and C. Rocha. A permutation related to the dynamics of a scalar parabolic PDE. J. Diff. Eqns. 91 (1991), 75-94.

[GaHa86] M. Gage and R.S. Hamilton. The heat equation shrinking convex plane curves. J. Diff. Geom. 23 (1986), 69-96.

[GaSl02] T. Gallay and S. Slijepčevič. Personal communication, (2002).

[GiHi96a] M. Giaquinta and S. Hildebrandt. Calculus of Variations 1. The Lagrangian Formalism. Springer-Verlag, Berlin, 1996.

[GiHi96b] M. Giaquinta and S. Hildebrandt. Calculus of Variations 2. The Hamiltonian Formalism. Springer-Verlag, Berlin, 1996.

[Go\&al00] M. Golubitsky, E. Knobloch, and I. Stewart. Target patterns and spirals in planar reaction-diffusion systems. J. Nonlinear Sci. 10 (2000), 333-354.

[Go\&al97] M. Golubitsky, V. LeBlanc, and I. Melbourne. Meandering of the spiral tip: an alternative approach. J. Nonl. Sci. 7 (1997), 557-586. 
[GoSc85] M. Golubitsky and D.G. Schaeffer. Singularities and Groups in Bifurcation Theory I . Springer-Verlag, 1985.

[Go\&al88] M. Golubitsky, I. Stewart, and D.G. Schaeffer. Singularities and Groups in Bifurcation Theory II. Springer-Verlag, New York, 1988.

[Go\&al98] A. Goryachev, H. Chaté, and R. Kapral. Synchronization defects and broken symmetry in spiral waves. Phys. Rev. Lett. 80 (1998), 873-876.

[Gr89] M. A. Grayson. Shortening embedded curves. Ann. Math. 129 (1989), 71111.

[GuHo83] J. Guckenheimer and P. Holmes. Nonlinear Oscillations, Dynamical Systems, and Bifurcations of Vector Fields. Springer-Verlag, New York, 1983.

[Ha\&al] G. Haas, M. Bär, and I.G. Kevrekidis et al. Observation of front bifurcations in controlled geometries: From one to two dimensions. Phys. Rev. Lett. 75 (1995), 3560-3563.

[Ha82] P.S. Hagan. Spiral waves in reaction-diffusion equations. SIAM J. Appl. Math. 42 (1982), 762-786.

[Ha69] J.K. Hale. Ordinary Differential Equations. John Wiley \& Sons, New York, 1969.

[Ha85] J.K. Hale. Flows on centre manifolds for scalar functional differential equations. Proc. R. Soc. Edinb., Sect. A 101 (1985), 193-201.

[Ha88] J.K. Hale. Asymptotic Behavior of Dissipative Systems. Math. Surv. 25. AMS Publications, Providence, 1988.

[HaRa92] J.K. Hale and G. Raugel. Reaction-diffusion equation on thin domains. J. Math. Pures Appl. 71 (1992), 33-95.

[Hä97] J. Härterich. Attractors of Viscous Balance Laws. Dissertation, Freie Universität Berlin, 1997.

[Hä98] J. Härterich. Attractors of viscous balance laws: Uniform estimates for the dimension. J. Diff. Eqns. 142 (1998), 188-211.

[Hä99] J. Härterich. Equilibrium solutions of viscous scalar balance laws with a convex flux. Nonlin. Diff. Eqns. Appl. 6 (1999), 413-436.

[HaMi91] H. Hattori and K. Mischaikow. A dynamical system approach to a phase transition problem. J. Diff. Eqns. 94 (1991), 340-378.

[He89] S. Heinze. Travelling waves for semilinear parabolic partial differential equations in cylindrical domains. Dissertation, Heidelberg, 1989.

[He81] D. Henry. Geometric Theory of Semilinear Parabolic Equations. Lect. Notes Math. 804, Springer-Verlag, New York, Berlin, Heidelberg, 1981.

[He85] D. Henry. Some infinite dimensional Morse-Smale systems defined by parabolic differential equations. J. Diff. Eqns. 59 (1985), 165-205.

[HeWi91] C. Henze and A. T. Winfree. A stable knotted singularity in an excitable medium. Int. J. Bif. Chaos 1 (1991), 891-922.

[Hi83] M. W. Hirsch. Differential equations and convergence almost everywhere in strongly monotone semiflows. J. Smoller, (ed.). In Nonlinear Partial Differential Equations. p. 267-285, AMS Publications, Providence, 1983.

[Hi85] M. W. Hirsch. Systems of differential equations that are competitive or cooperative II. Convergence almost everywhere. SIAM J. Math. Analysis 16 (1985), 423-439.

[Hi88] M. W. Hirsch. Stability and convergence in strongly monotone dynamical systems. Crelle J. reine angew. Math. 383 (1988), 1-58.

[Hi\&al77] M. W. Hirsch, C.C. Pugh, and M. Shub. Invariant Manifolds. SpringerVerlag, Berlin, 1977. 
[IoMi91] G. Iooss and A. Mielke. Bifurcating time-periodic solutions of NavierStokes equations in infinite cylinders. J. Nonlinear Science 1 (1991), 107-146.

[JäLu92] W. Jäger and S. Luckhaus. On explosions of solutions to a system of partial differential equations modelling chemotaxis. Trans. Am. Math. Soc., 329 (1992), 819-824.

[Ja\&al88] W. Jahnke, C. Henze, and A.T. Winfree. Chemical vortex dynamics in the 3-dimensional excitable media. Nature 336 (1988), 662-665.

[Ja\&al89] W. Jahnke, W.E. Skaggs, and A.T. Winfree. Chemical vortex dynamics in the Belousov-Zhabotinskii reaction and in the two-variable Oregonator model. J. Chem. Phys. 93 (1989), 740-749.

[Ka66] T. Kato. Perturbation Theory for Linear Operators. Springer, Berlin, Heidelberg, New York, 1966.

[KaHa95] A. Katok and B. Hasselblatt. Introduction to the modern theory of $d y$ namical systems. With a supplementary chapter by Katok and Leonardo Mendoza. Encyclopedia of Mathematics and its Applications 54, Cambridge University Press, Cambridge, 1995.

[KaHa02] A. Katok and B. Hasselblatt (eds.) Handbook of Dynamical Systems 1, Elsevier, Amsterdam. to appear 2002.

[Ka87] L.H. Kauffman. On Knots. Princeton University Press, New Jersey, 1987.

[Ke92] J.P. Keener. The core of the spiral. SIAM J. Appl. Math. 52 (1992), 13701390.

[KeTy92] J.P. Keener and J.J. Tyson. The dynamics of scroll waves in excitable media. SIAM Rev., 34 (1992), 1-39.

[Ki82] K. Kirchgässner. Wave-solutions of reversible systems and applications. $J$. Differential Equations 45 (1982), 113-127.

[Ki00] S.V. Kiyashko. The generation of stable waves in faraday experiment. 2000 Int. Symp. Nonlinear Theory and its Applications, 2000.

[KoHo73] N. Kopell and L.N. Howard. Plane wave solutions to reaction-diffusion equations. Studies in Appl. Math. 52 (1973), 291-328.

[KoHo81] N. Kopell and L.N. Howard. Target patterns and spiral solutions to reaction-diffusion equations with more than one space dimension. Adv. Appl. Math. 2 (1981), 417-449.

[Ko02] V.V. Kozlov. General Theory of Vortices. Enc. Math. Sc. 67, Dynamical Systems X. Springer-Verlag, New York, 2002.

[Kr90] M. Krupa. Bifurcations of relative equilibria. SIAM J. Math. Analysis 21 (1990), 1453-1486.

[KuMa83] M. Kubiček and M. Marek. Computational Methods in Bifurcation Theory and Dissipative Structures. Springer-Verlag, New York, 1983.

[Ku95] Y.A. Kuznetsov. Elements of Applied Bifurcation Theory. Springer-Verlag, Berlin, 1995.

[La91] O.A. Ladyzhenskaya. Attractors for Semigroups and Evolution Equations. Cambridge University Press, 1991.

[LaLi59] L.D. Landau and E.M. Lifschitz. Fluid Mechanics. Pergamon Press, London, 1959.

[Li90] X.-B. Lin. Using Melnikov's method to solve Shilnikov's problems. Proc. Roy. Soc. Edinburgh, 116A (1990), 295-325.

[Ou\&al] G. Li, Q. Ouyang, V. Petrov, and H. L. Swinney. Transition from simple rotating chemical spirals to meandering and traveling spirals. Phys. Rev. Lett. 77 (1996), 2105-2108. 
[Lo04] A.E.H. Love. A Treatise on the Mathematical Theory of Elasticity. Dover Publications, New-York, 1904.

[MP88] J. Mallet-Paret. Morse decompositions for delay-differential equations. J. Diff. Eqns. 72 (1988), 270-315.

[MPSm90] J. Mallet-Paret and H. Smith. The Poincaré-Bendixson theorem for monotone cyclic feedback systems. J. Diff. Eqns. 4 (1990), 367-421.

[MPSe96a] J. Mallet-Paret and G.R. Sell. The Poincaré-Bendixson theorem for monotone cyclic feedback systems with delay. J. Diff. Eqns. 125 (1996), 441489.

[MPSe96b] J. Mallet-Paret and G.R. Sell. Systems of differential delay equations: Floquet multipliers and discrete Lyapunov functions. J. Diff. Eqns. 125 (1996), 385-440.

[MaPa97] A.F.M. Maree and A.V. Panfilov. Spiral breakup in excitable tissue due to lateral instability. Phys. Rev. Lett. 78 (1997), 1819-1822.

[Ma78] H. Matano. Convergence of solutions of one-dimensional semilinear parabolic equations. J. Math. Kyoto Univ., 18 (1978), 221-227.

[Ma79] H. Matano. Asymptotic behavior and stability of solutions of semilinear diffusion equations. Publ. Res. Inst. Math. Sc. Kyoto Univ. 15 (1979), 401454.

[Ma82] H. Matano. Nonincrease of the lap-number of a solution for a onedimensional semi-linear parabolic equation. J. Fac. Sci. Univ. Tokyo Sec. IA, 29(1982), 401-441.

[Ma86] H. Matano. Strongly order-preserving local semi-dynamical systems - theory and applications. In Semigroups, Theory and Applications. H. Brezis, M.G. Crandall, F. Kappel (eds.), 178-189. John Wiley \& Sons, New York, 1986.

[Ma87] H. Matano. Strong comparison principle in nonlinear parabolic equations. In Nonlinear Parabolic Equations: Qualitative Properties of Solutions, L. Bocardo, A. Tesei (eds.), 148-155. Pitman Res. Notes Math. Ser. 149 (1987).

[Ma88] H. Matano. Asymptotic behavior of solutions of semilinear heat equations on $S^{1}$. In Nonlinear Diffusion Equations and their Equilibrium States II . W.-M. Ni, L.A. Peletier, J. Serrin (eds.). 139-162. Springer-Verlag, New York, 1988.

[MaNa97] H. Matano and K.-I. Nakamura. The global attractor of semilinear parabolic equations on $S^{1}$. Discr. Contin. Dyn. Syst. 3 (1997), 1-24.

[MaWi89] J. Mawhin and M. Willem. Critical Point Theory and Hamiltonian Systems. Springer-Verlag, New York, 1989.

[Mi91] A. Mielke. Hamiltonian and Lagrangian Flows on Center Manifolds. Springer-Verlag, Berlin, 1991.

[Mi94] A. Mielke. Essential manifolds for elliptic problems in infinite cylinders. $J$. Diff. Eqns., 110 (1994), 322-355.

[Mi97] A. Mielke. Instability and stability of rolls in the Swift-Hohenberg equation. Comm. Math. Phys. 189 (1997), 829-853.

[MiSc96] A. Mielke and G. Schneider. Derivation and justification of the complex Ginzburg-Landau equation as a modulation equation. Dynamical systems and probabilistic methods in partial differential equations (Berkeley, CA, 1994), 191-216, Lectures in Appl. Math. 31, Amer. Math. Soc., Providence, RI, 1996.

[MiZy91] A.S. Mikhailov and V.S. Zykov. Kinematical theory of spiral waves in excitable media: comparison with numerical simulations. Physica D 52 (1991), 379-397.

[Mo73] J. Moser. Stable and Random Motions in Dynamical Systems. Princeton University Press, New York, 1973. 
[MüZy94] S.C. Müller and V.S. Zykov. Simple and complex spiral wave dynamics. Phil. Trans. Roy. Soc. Lond. A 347 (1994), 677-685.

[Na90] N.S. Nadirashvili. On the dynamics of nonlinear parabolic equations. Soviet Math. Dokl. 40 (1990), 636-639.

[Ne\&al] S. Nettesheim, A. von Oertzen, H.H. Rotermund, and G. Ertl. Reaction diffusion patterns in the catalytic CO-oxidation on $\mathrm{Pt}(110)$ - front propagation and spiral waves. J. Chem. Phys. 98 (1993), 9977-9985.

[OgNa00] T. Ogiwara and K.-I. Nakamura. Spiral traveling wave solutions of some parabolic equations on annuli. In Nonlinear Analysis, Josai Math. Monogr., Nishizawa, Kiyoko (ed.) 2 (2000), 15-34.

[O102] W.M. Oliva. Morse-Smale semiflows. Openess and A-stability. Comm. Fields Inst. (2002), in press.

[Pa61] R. S. Palais. On the existence of slices for actions of non-compact Lie groups. Ann. of Math. 73 (1961), 295-323.

[Pa69] J. Palis. On Morse-Smale dynamical systems. Topology 8 (1969), 385-404.

[PaSm70] J. Palis and S. Smale. Structural stability theorems. In Global Analysis. Proc. Symp. in Pure Math. vol. XIV. AMS, Providence, 1970. S. Chern, S. Smale (eds.).

[Pa88] K.J. Palmer. Exponential dichotomies and Fredholm operators. Proc. Amer. Math. Soc. 104 (1988), 149-156.

[PaWi85] A.V. Panfilov and A. T. Winfree. Dynamical simulations of twisted scroll rings in 3-dimensional excitable media. Physica D 17 (1985), 323-330.

[Pa83] A. Pazy. Semigroups of Linear Operators and Applications to Partial Differential Equations. Springer-Verlag, New York, 1983.

[Pe\&a191] V. Perez-Munuzuri, R. Aliev, B. Vasiev, V. Perez-Villar, and V. I. Krinsky. Super-spiral structure in an excitable medium. Nature 353 (1991) 740-742.

[Pe\&al93] V. Perez-Munuzuri, M. Gomez-Gesteira, and V. Perez-Villar. A geometrical-kinematical approach to spiral wave formation: Super-spiral waves. Physica D 64 (1993), 420-430.

[Pe\&al97] D. Peterhof, A. Scheel, and B. Sandstede. Exponential dichotomies for solitary wave solutions of semilinear elliptic equations on infinite cylinders. $J$. Diff. Eqns. 140 (1997), 266-308.

[PlBo96] B.B. Plapp and E. Bodenschatz. Core dynamics of multiarmed spirals in Rayleigh-Bénard convection. Physica Scripta 67 (1996), 111-117.

[Po89] P. Poláčik. Convergence in strongly monotone flows defined by semilinear parabolic equations. J. Diff. Eqs. 79 (1989), 89-110.

[Po95] P. Poláčik. High-dimensional $\omega$-limit sets and chaos in scalar parabolic equations. J. Diff. Eqns., 119 (1995), 24-53.

[Po02] P. Poláčik. Parabolic equations: Asymptotic behavior and dynamics on invariant manifolds. In Handbook of Dynamical Systems, Vol. 2. B. Fiedler (ed.), Elsevier, Amsterdam, 2002. In press.

[Po33] G. Polya. Qualitatives über Wärmeaustausch. Z. Angew. Math. Mech. 13 (1933), 125-128,.

[Po92] G. Pospiech. Eigenschaften, Existenz und Stabilität von travelling wave Lösungen zu einem System von Reaktions-Diffusions-Gleichungen. Dissertation, Universität Heidelberg, 1992.

[PrRy98a] M. Prizzi and K.P. Rybakowski. Complicated dynamics of parabolic equations with simple gradient dependence. Trans. Am. Math. Soc. 350 (1998), $3119-3130$. 
[PrRy98b] M. Prizzi and K.P. Rybakowski. Inverse problems and chaotic dynamics of parabolic equations on arbitrary spatial domains. J. Diff. Eqns. 142 (1998), $17-53$.

[PrWe67] M.H. Protter and H.F. Weinberger. Maximum Principles in Differential Equations. Prentice Hall, Englewood Cliffs, New Jersey, 1967.

[Ra02] G. Raugel. Global attractors. In Handbook of Dynamical Systems, Vol. 2. B. Fiedler (ed.), Elsevier, Amsterdam, 2002. In press.

[ReSi78] M. Reed and B. Simon. Methods of Modern Mathematical Physics IV. Academic Press, 1978.

[RoSa95] J. Robbin and D. Salamon. The spectral flow and the Maslov index. Bull. London Math. Soc. 27 (1995), 1-33.

[Ro85] C. Rocha. Generic properties of equilibria of reaction-diffusion equations with variable diffusion.Proc. R. Soc. Edinb. A 101 (1985), 45-55.

[Ro91] C. Rocha. Properties of the attractor of a scalar parabolic PDE. J. Dyn. Differ. Equations 3 (1991), 575-591.

[Sa93a] B. Sandstede. Verzweigungstheorie homokliner Verdopplungen. Dissertation, Universität Stuttgart, 1993.

[Sa93b] B. Sandstede. Asymptotic behavior of solutions of non-autonomous scalar reaction-diffusion equations. In Conf. Proceeding International Conference on Differential Equations, Barcelona 1991, C. Perello, C. Simo, and J. SolaMorales (eds.), 888-892, World Scientific, Singapore, 1993.

[SaFi92] B. Sandstede and B. Fiedler. Dynamics of periodically forced parabolic equations on the circle. Ergod. Theor. Dynam. Sys. 12 (1992), 559-571.

[SaSc99] B. Sandstede and A. Scheel. Essential instability of pulses and bifurcations to modulated travelling waves. Proc. Roy. Soc. Edinburgh. A 129 (1999), 12631290.

[SaSc00a] B. Sandstede and A. Scheel.Gluing unstable fronts and backs together can produce stable pulses. Nonlinearity 13 (2000), 1465-1482.

[SaSc00b] B. Sandstede and A. Scheel. Spectral stability of modulated travelling waves bifurcating near essential instabilities. Proc. R. Soc. Edinburgh A 130 (2000), 419-448.

[SaSc00c] B. Sandstede and A. Scheel. Absolute and convective instabilities of waves on unbounded and large bounded domains. Physica D 145 (2000), 233277.

[SaSc00d] B. Sandstede and A. Scheel. Absolute versus convective instability of spiral waves. Phys. Rev. E. 62 (2000), 7708-7714.

[SaSc01a] B. Sandstede and A. Scheel. Super-spiral structures of meandering and drifting spiral waves. Phys. Rev. Lett. 86 (2001), 171-174.

[SaSc01b] B. Sandstede and A. Scheel. Essential instabilities of fronts: bifurcation and bifurcation failure. Dynamical Systems: An International Journal 16 (2001), 1-28.

[SaSc01c] B. Sandstede and A. Scheel. On the stability of periodic travelling waves with large spatial period. J. Diff. Eqns. 172 (2001), 134-188.

[SaSc01d] B. Sandstede and A. Scheel. On the structure of spectra of modulated travelling waves. Math. Nachr. 232 (2001), 39-93.

[SaSc02a] B. Sandstede and A. Scheel. Nonlinear convective stability and instability - the role of absolute spectra and nonlinearities. In preparation (2002).

[SaSc02b] B. Sandstede and A. Scheel. Instabilities of spiral waves in large disks. In preparation (2002). 
[Sa\&al97a] B. Sandstede, A. Scheel, and C. Wulff. Center manifold reduction for spiral wave dynamics. C. R. Acad. Sci. Paris, Série I 324 (1997), 153-158.

[Sa\&al97b] B. Sandstede, A. Scheel, and C. Wulff. Dynamics of spiral waves on unbounded domains using center-manifold reduction. J. Diff. Eqns. 141 (1997), 122-149.

[Sa\&a199] B. Sandstede, A. Scheel, and C. Wulff. Bifurcations and dynamics of spiral waves. J. Nonlinear Science 9 (1999), 439-478.

[Sc90] R. Schaaf. Global Solution Branches of Two Point Boundary Value Problems. Springer-Verlag, New York, 1990.

[Sc96] A. Scheel. Existence of fast travelling waves for some parabolic equations a dynamical systems approach. J. Dyn. Diff. Eqns. 8 (1996), 469-548.

[Sc97] A. Scheel. Subcritical bifurcation to infinitely many rotating waves. J. Math. Anal. Appl. 215 (1997), 252-261.

[Sc98] A. Scheel. Bifurcation to spiral waves in reaction-diffusion systems. SIAM J. Math. Anal. 29 (1998), 1399-1418.

[Sc01] A. Scheel.Radially symmetric patterns of reaction-diffusion systems. Preprint 2001.

[Sc98b] G. Schneider. Hopf bifurcation in spatially extended reaction-diffusion systems. J. Nonlinear Sci. 8 (1998), 17-41.

[Sc98c] G. Schneider. Nonlinear diffusive stability of spatially periodic solutions abstract theorem and higher space dimensions. Tohoku Math. Publ. 8 (1998), 159-167.

[Si89] Ya.G. Sinai. Ergodic theory with applications to dynamical systems and statistical mechanics. Enc. Math. Sc. 2, Dynamical Systems II. Springer-Verlag, Berlin, 1989.

[SkSw91] G. S. Skinner and H. L. Swinney. Periodic to quasiperiodic transition of chemical spiral rotation. Physica D 48 (1991), 1-16.

[Sm95] H. Smith. Monotone Dynamical Systems: An Introduction to the Theory of Competitive and Cooperative Systems. AMS, Providence, 1995.

[Sm83] J. Smoller. Shock Waves and Reaction-Diffusion Equations. SpringerVerlag, New York, 1983.

[St00] A. Steven. The derivation of chemotaxis equations as limit dynamics of moderately interacting stochastic many-particle systems. SIAM J. Appl. Math., 61 (2000), 183-212.

[St90] M. Struwe. Variational Methods. Springer-Verlag, Berlin, 1990.

[St36] C. Sturm. Sur une classe d'équations à différences partielles. J. Math. Pure Appl. 1 (1836), 373-444,.

[Ta74] F. Takens. Singularities of vector fields. Publ. IHES, 43 (1974), 47-100.

[Ta79] H. Tanabe. Equations of Evolution. Pitman, Boston, 1979.

[Te88] R. Temam. Infinite-Dimensional Dynamical Systems in Mechanics and Physics. Springer-Verlag, New York, 1988.

[ToKn98] S.M. Tobias and E. Knobloch. Breakup of spiral waves into chemical turbulence. Phys. Rev. Lett. 80 (1998), 4811-4814.

[Tu52] A. Turing. The chemical basis of morphogenesis. Phil. Trans. Roy. Soc. B 237 (1952), 37-72.

[TySt91] J.J. Tyson and S.H. Strogatz. The differential geometry of scroll waves. Int. J. Bif. Chaos, 1 (1991), 723-744.

[Uh76] K. Uhlenbeck. Generic properties of eigenfunctions. Amer. J. Math., 98 (1976), 1059-1078. 
[Un\&al93] Zs. Ungvarai-Nagy, J. Ungvarai, and S.C. Müller. Complexity in spiral wave dynamics. Chaos 3 (1993), 15-19.

[Va82] A. Vanderbauwhede. Local Bifurcation and Symmetry. Pitman, Boston, 1982.

[Va89] A. Vanderbauwhede. Center manifolds, normal forms and elementary bifurcations. Dynamics Reported 2 (1989), 89-169.

[Wa70] W. Walter. Differential and Integral Inequalities. Springer-Verlag, New York, 1970.

[WiRo46] N. Wiener and A. Rosenblueth. The mathematical formulation of the problem of conduction of impulses in a network of connected excitable elements, specifically in cardiac muscle. Arch. Inst. Cardiol. Mexico 16 (1946), 205-265.

[Wi72] A. T. Winfree. Spiral waves of chemical activity. Science, 175 (1972), 634636.

[Wi73] A. T. Winfree. Scroll-shaped waves of chemical activity in three dimensions. Science 181 (1973), 937-939.

[Wi87] A. T. Winfree. When Time Breaks Down. Princeton University Press, Princeton, NJ, 1987.

[Wi91] A. T. Winfree. Varieties of spiral wave behavior: An experimentalist's approach to the theory of excitable media. Chaos 1 (1991), 303-334.

[Wi95] A. T. Winfree. Persistent tangles of vortex rings in excitable media. Physica D 84 (1995), 126-147.

[Wi01] A. T. Winfree. The geometry of biological time. Biomathematics 8, Springer-Verlag, Berlin-New York, 2001.

[Wi\&al95] A. T. Winfree, E.M. Winfree, and M. Seifert. Organizing centers in a cellular excitable medium. Physica D, 17 (1995), 109-115.

[Wo98] Matthias Wolfrum. Geometry of Heteroclinic Cascades in Scalar Semilinear Parabolic Equations. Dissertation, Freie Universität Berlin, 1998.

[Wo02a] M. Wolfrum. Personal communication, (2002).

[Wo02b] M. Wolfrum. A sequence of order relations, encoding heteroclinic connections in scalar parabolic PDEs. J. Diff. Eqns., to appear (2002).

[Wu96] C. Wulff. Theory of Meandering and Drifting Spiral Waves in ReactionDiffusion Systems. Dissertation, Berlin, 1996.

[Wu\&al01] C. Wulff, J. Lamb, and I. Melbourne. Bifurcation from relative periodic solutions. Ergodic Theory Dynam. Systems 21 (2001), 605-635.

[Ya\&al98] H. Yagisita, M. Mimura, and M. Yamada. Spiral wave behaviors in an excitable reaction-diffusion system on a sphere. Physica D 124 (1998), 126-136.

[Ze85] E. Zeidler. Nonlinear functional analysis and its applications. III: Variational methods and optimization. Springer-Verlag, New York, 1985.

[Ze93] E. Zeidler. Nonlinear functional analysis and its applications. Volume I: Fixed-point theorems. Springer-Verlag, New York, 1993.

[Ze68] T.I. Zelenyak. Stabilization of solutions of boundary value problems for a second order parabolic equation with one space variable. Diff. Eqns. 4 (1968), $17-22$.

[ZhOu00] L. Q. Zhou and Q. Ouyang. Experimental studies on long-wavelength instability and spiral breakup in a reaction-diffusion system. Phys. Rev. Lett. 85 (2000), 1650-1653. 